\title{
Nickel-Catalyzed Allylic Defluorinative Cross-Electrophile Coupling with Cycloalkyl Silyl Peroxides as the Alkyl Source
}

\author{
Haiyan Dong, ${ }^{\dagger}$ Zhiyang Lin, ${ }^{\dagger}$ and Chuan Wang ${ }^{*}, \dagger$ \\ ${ }^{\dagger}$ Department of Chemistry, University of Science and Technology of China, Hefei, Anhui 230026, \\ P. R. China \\ $\$$ Center for Excellence in Molecular Synthesis of CAS, Hefei, Anhui 230026, P. R. China \\ E-mail: chuanw@ustc.edu.cn
}

\section{Contents}

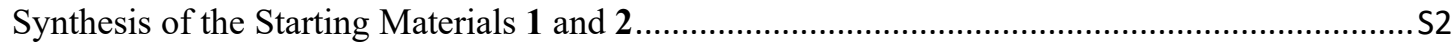

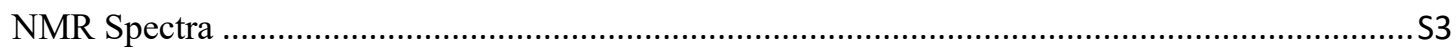




\section{Synthesis of the Starting Materials 1 and 2}

The trifluoromethyl alkenes $\mathbf{1 a},{ }^{1} \mathbf{1 b},{ }^{2} \mathbf{1 c},{ }^{3} \mathbf{1 d},{ }^{1} \mathbf{1 e},{ }^{2} \mathbf{1 f}-\mathbf{h},{ }^{4} \mathbf{1 i},{ }^{5} \mathbf{1 j},{ }^{4} \mathbf{1 k},{ }^{5} \mathbf{1 l}-\mathbf{n},{ }^{2} \mathbf{1 o}-\mathbf{1} \mathbf{p},{ }^{4}$ $\mathbf{1 q},{ }^{6} \mathbf{1 r},{ }^{2} \mathbf{1 s},{ }^{4} \mathbf{1 t},{ }^{2} \mathbf{1 u}-\mathbf{1} \mathbf{v}^{4}$, and $\mathbf{1} \mathbf{w}^{2}$, and the silyl peroxides $\mathbf{2 a}-\mathbf{r}^{8}$ and $\mathbf{2} \mathbf{s}^{\mathbf{}}{ }^{9}$ were synthesized following the reported procedures.

1. Ichitsuka, T.; Fujita, T.; Arita,T.; Ichikawa, J. Double C-F Bond Activation through $\beta$-Fluorine Elimination: Nickel-Mediated [3+2] Cycloaddition of 2-Trifluoromethyl-1alkenes with Alkynes. Angew. Chem. Int. Ed. 2014, 53, 7564-7568.

2. Lin, Z.; Lan, Y.; Wang, C. Synthesis of gem-Difluoroalkenes via Nickel-Catalyzed Reductive C-F and C-O Bond Cleavage. ACS Catal. 2019, 9, 775-780.

3. Li, Y.; Zhao, B.; Dai, K.; Lan.; Tu, D.; Lu, J. Suzuki-Miyaura Reaction of Fluorinated Vinyl Chloride: a New Approach for Synthesis $\alpha$ and $\alpha, \beta$-Trifluoromethylstyrenes. Y. Tetrahedron 2016, 72, 5684-5690.

4. Lan, Y.; Yang, F.; Wang, C. Synthesis of gem-Difluoroalkenes via Nickel-Catalyzed Allylic Defluorinative Reductive Cross-Coupling. ACS Catal. 2018, 8, 9245-9251.

5. Jin, Y.; Wu, J.; Lin, Z.; Lan, Y.; Wang, C. Merger of C-F and C-N Bond Cleavage in Cross-Electrophile Coupling for the Synthesis of gem-Difluoroalkenes. Org. Lett. 2020, 22, 5347-5352.

6. Guo, Y.; Wu, Y.; Wang, R.; Lan.; Song, H.; Liu, Y.; Wang, Q. Photoredox/Hydrogen Atom Transfer Cocatalyzed $\mathrm{C}-\mathrm{H}$ Difluoroallylation of Amides, Ethers, and Alkyl Aldehydes. Org. Lett. 2021, 23, 2353-2358.

7. Lin, Z.; Lan, Y.; Wang, C . Reductive Allylic Defluorinative Cross-Coupling Enabled by Ni/Ti Cooperative Catalysis. Org. Lett. 2019, 21, 8316-8322.

8. Yang, J.; Chen, L.; Yang, F.; Li, P.; Guo, L. Copper-Catalyzed Borylation of Cycloalkylsilyl Peroxides via Radical C-C bond Cleavage. Org. Chem. Front. 2019, 6, 2792-2795.

9. Seihara, T.; Sakurai, S.; Kato, T.; Sakamoto, R.; Maruoka, K. Synthesis of Functionalized Organoboron/Silicon Compounds by Copper-Catalyzed Coupling of Alkylsilyl Peroxides and Diboron/ Silylborane Reagents. Org. Lett. 2019, 21, 2477-2481. 


\section{NMR Spectra}

${ }^{1} \mathrm{H}$ NMR-spectrum (400 MHz, $\mathrm{CDCl}_{3}$ ) of 3aa

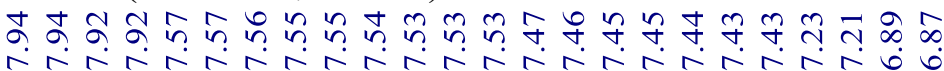

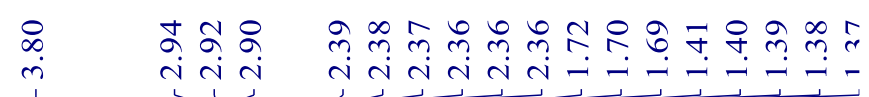
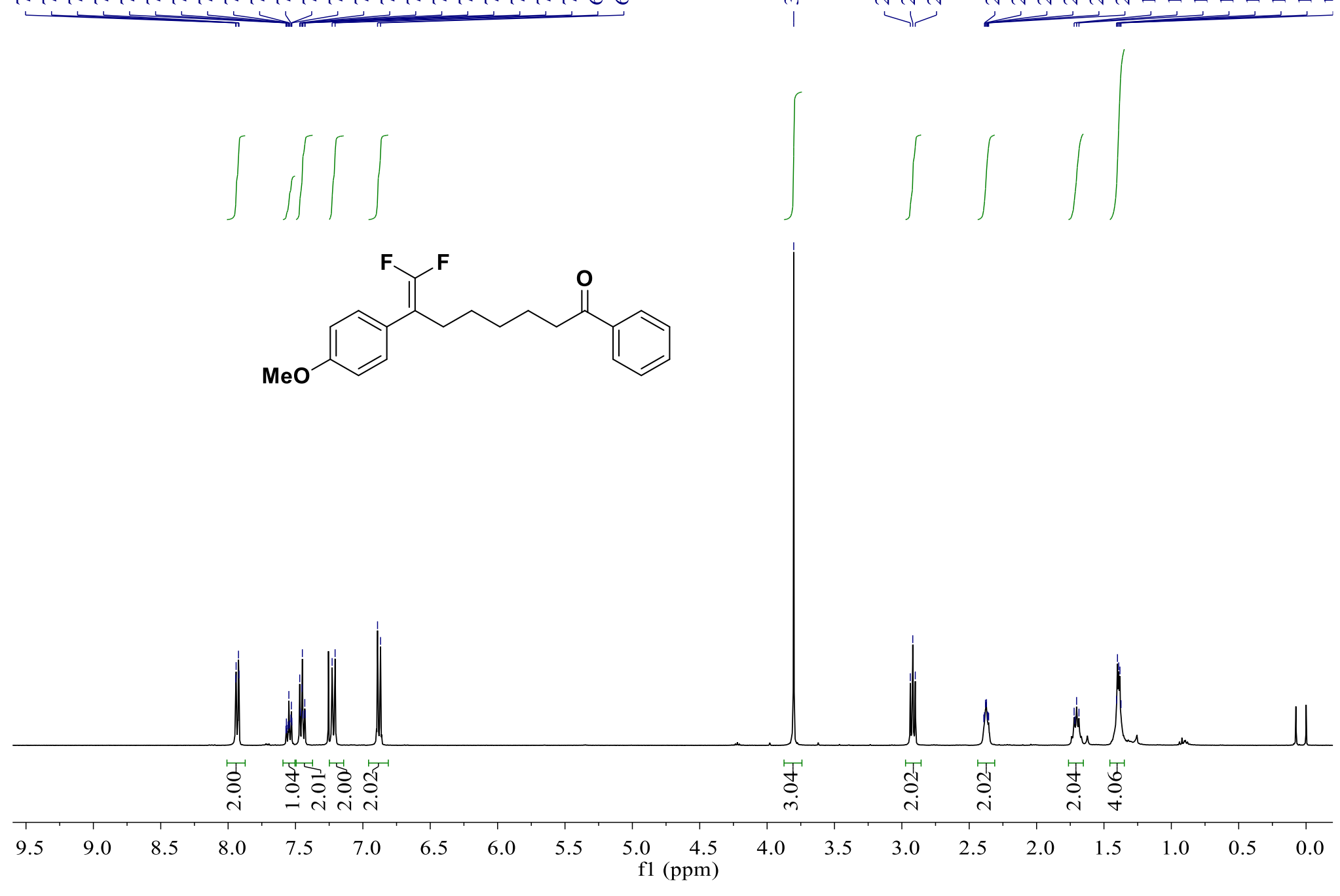
${ }^{13} \mathrm{C}\left\{{ }^{1} \mathrm{H}\right\}$ NMR-spectrum $\left(101 \mathrm{MHz}, \mathrm{CDCl}_{3}\right)$ of $\mathbf{3 a a}$

\begin{tabular}{|c|c|}
\hline & 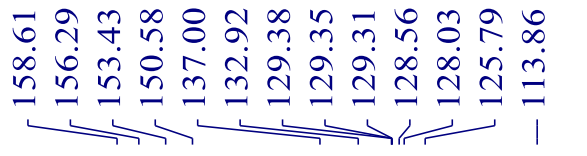 \\
\hline
\end{tabular}
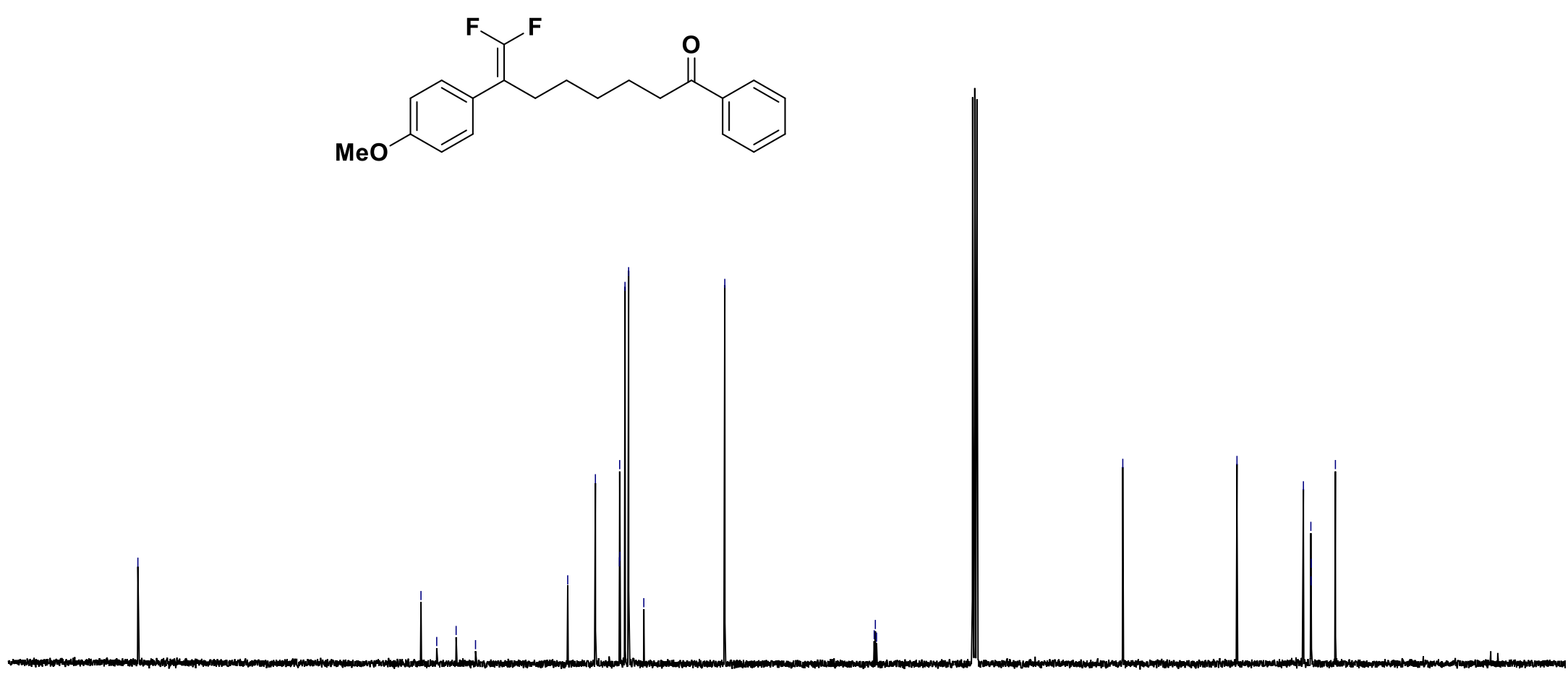

$\begin{array}{lllllllllllllllllllllll}210 & 200 & 190 & 180 & 170 & 160 & 150 & 140 & 130 & 120 & \begin{array}{c}110 \\ \mathrm{f} 1(\mathrm{ppm})\end{array} & 90 & 80 & 70 & 60 & 50 & 40 & 30 & 20 & 10 & 0 & -\end{array}$ 
${ }^{19} \mathrm{~F}$ NMR-spectrum $\left(376 \mathrm{MHz}, \mathrm{CDCl}_{3}\right)$ of $\mathbf{3 a a}$

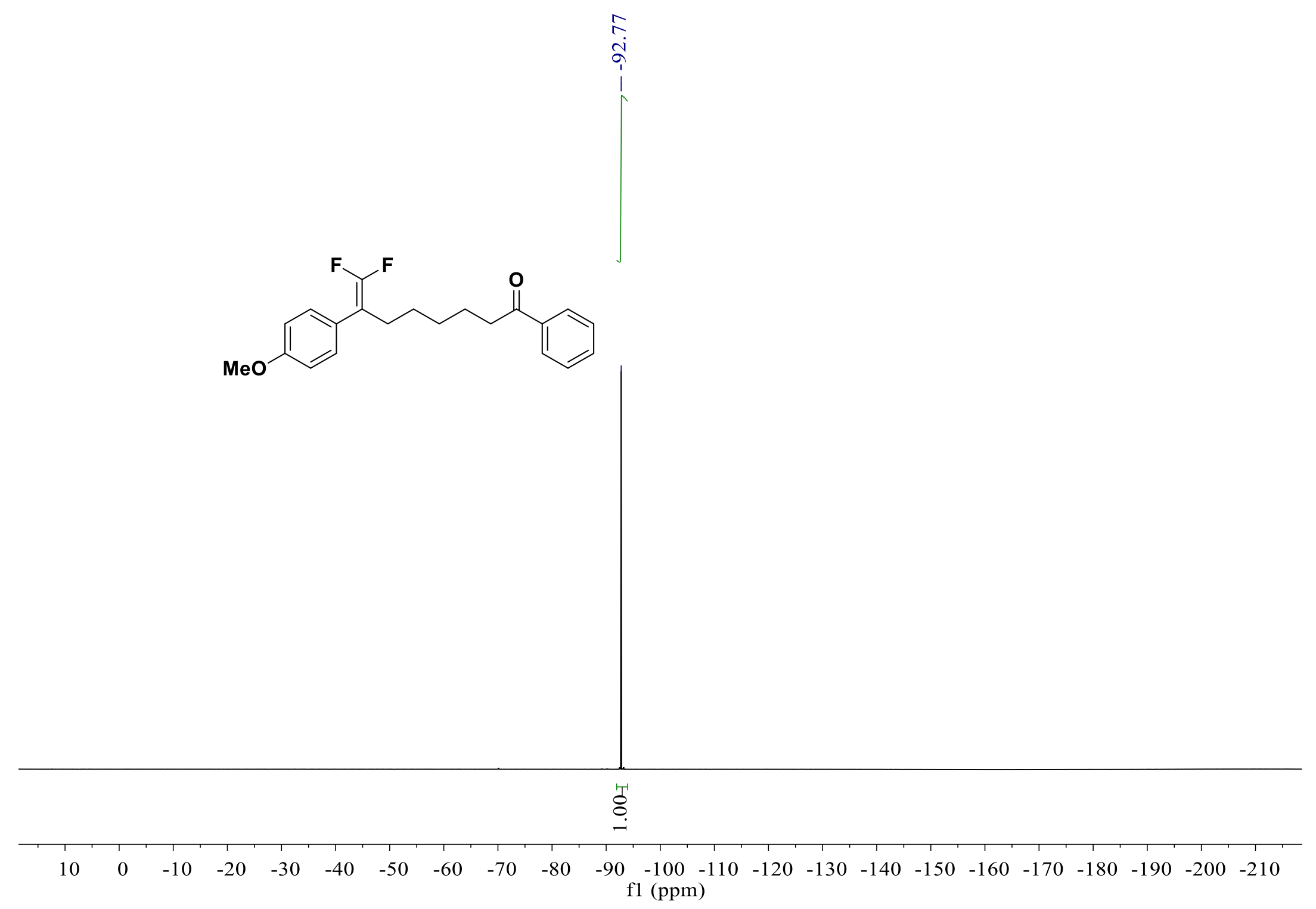




\section{${ }^{1} \mathrm{H}$ NMR-spectrum $\left(500 \mathrm{MHz}, \mathrm{CDCl}_{3}\right)$ of $\mathbf{3 b a}$}

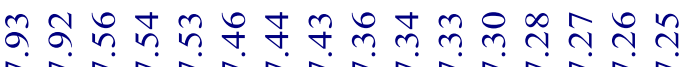

rarararar
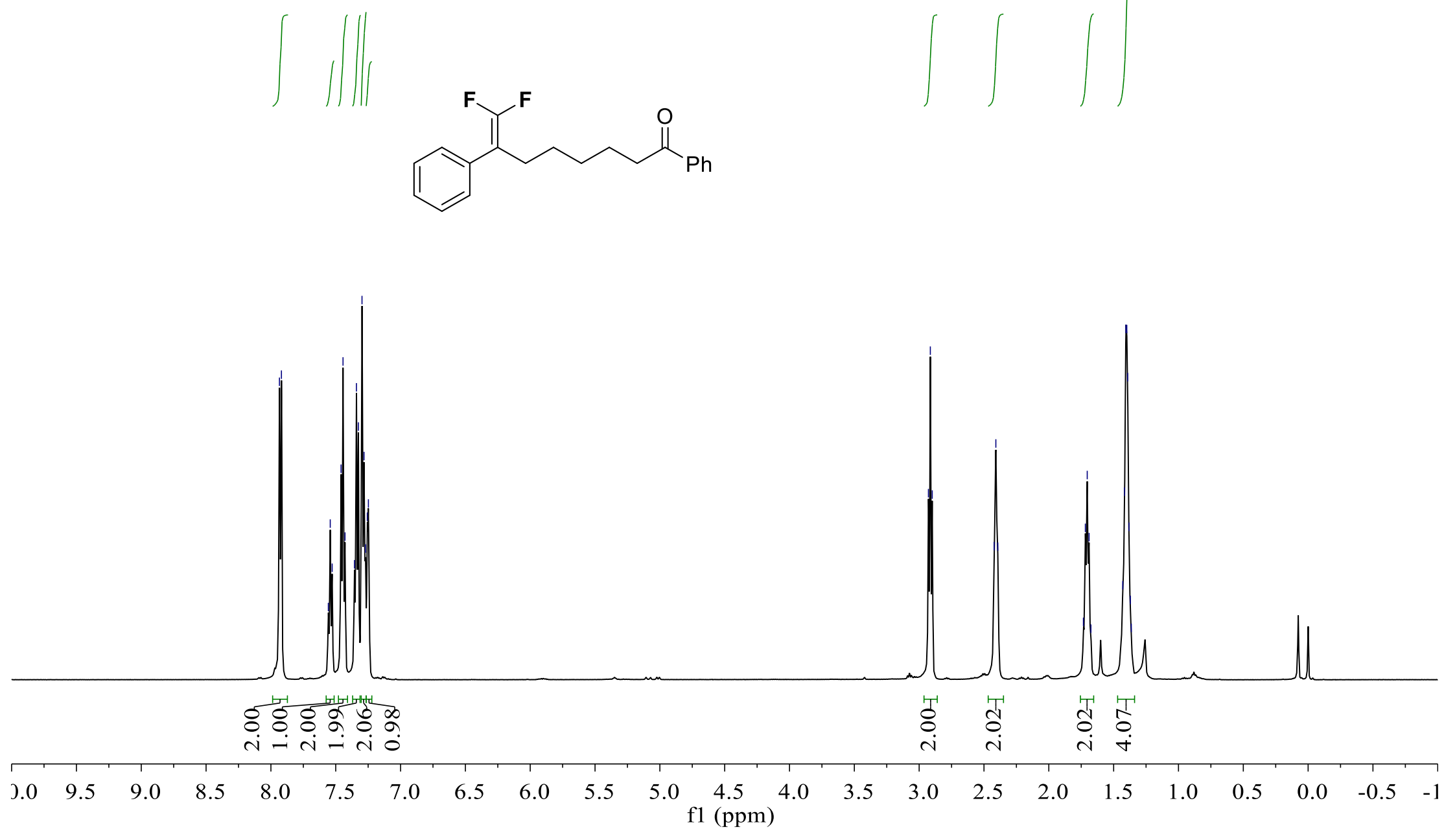

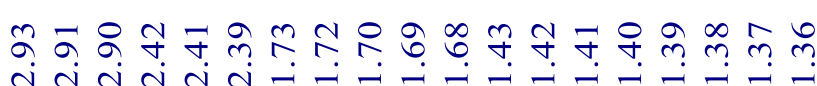
ง

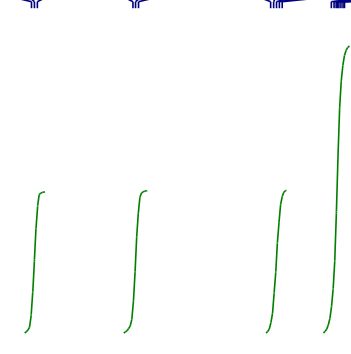


${ }^{13} \mathrm{C}\left\{{ }^{1} \mathrm{H}\right\}$ NMR-spectrum $\left(126 \mathrm{MHz}, \mathrm{CDCl}_{3}\right)$ of $\mathbf{3 b a}$
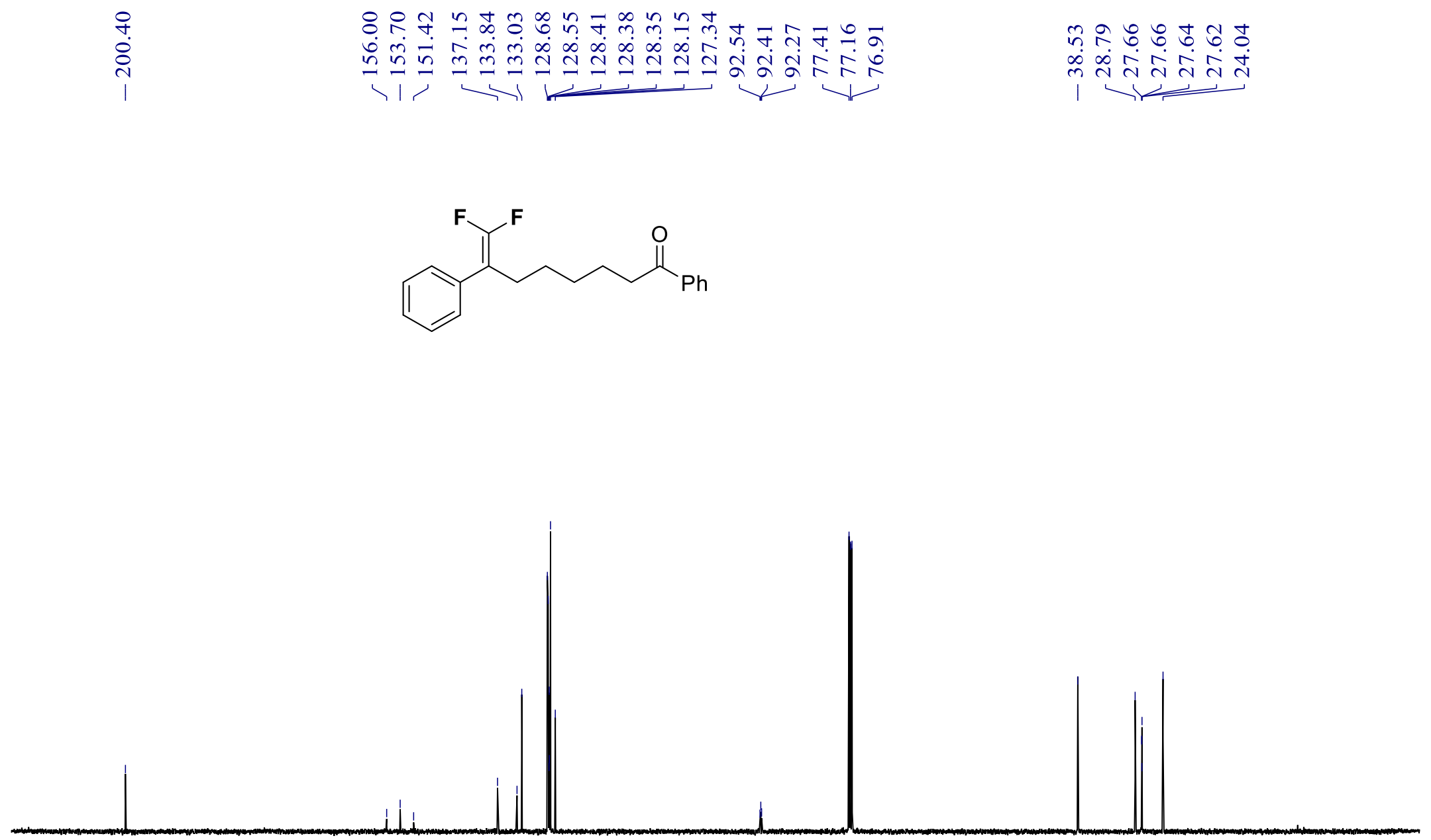

$\begin{array}{llllllllllllllllllllllll}210 & 200 & 190 & 180 & 170 & 160 & 150 & 140 & 130 & 120 & 110 & 100 & 90 & 80 & 70 & 60 & 50 & 40 & 30 & 20 & 10 & 0 & -10\end{array}$ 
${ }^{19} \mathrm{~F}$ NMR-spectrum $\left(400 \mathrm{MHz}, \mathrm{CDCl}_{3}\right)$ of $\mathbf{3 b a}$
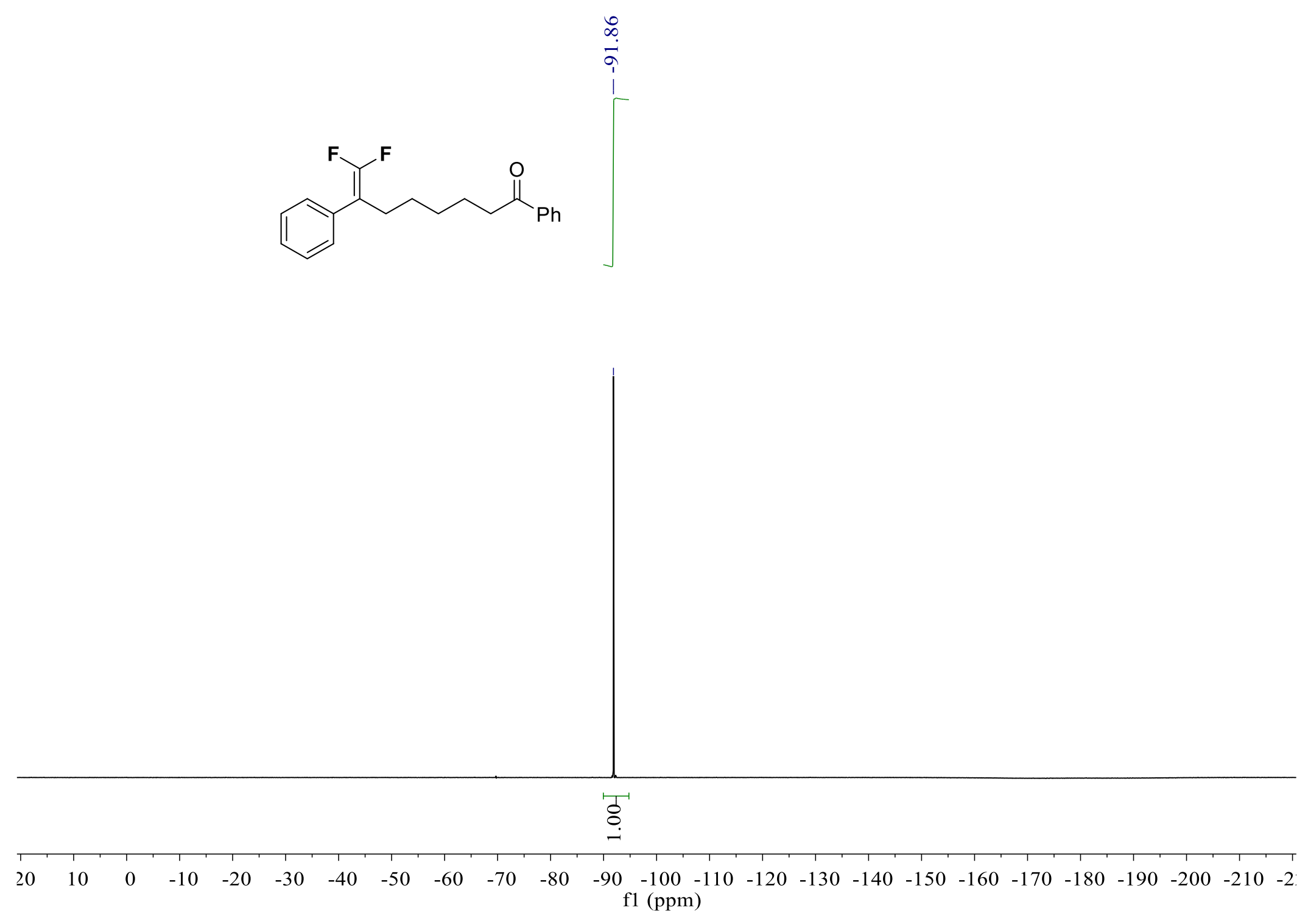


\section{${ }^{1} \mathrm{H}$ NMR-spectrum $\left(400 \mathrm{MHz}, \mathrm{CDCl}_{3}\right)$ of $\mathbf{3 c a}$}

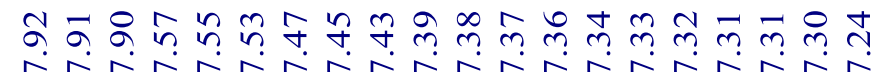

rin

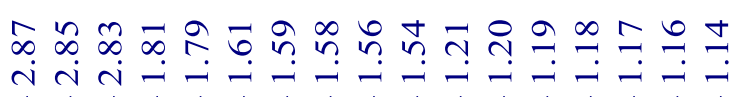
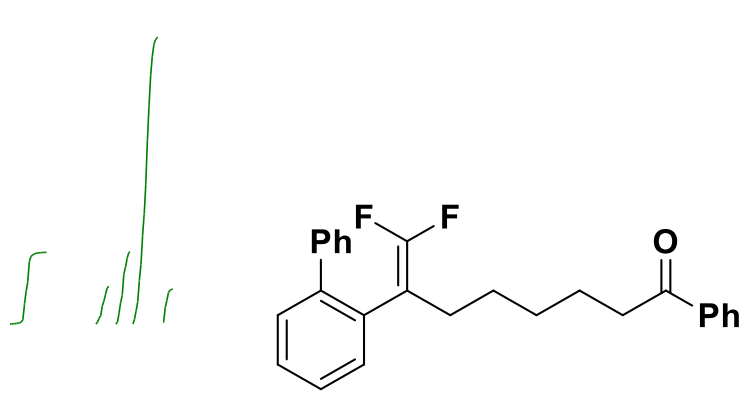

$\sqrt{n}=-2-3=$

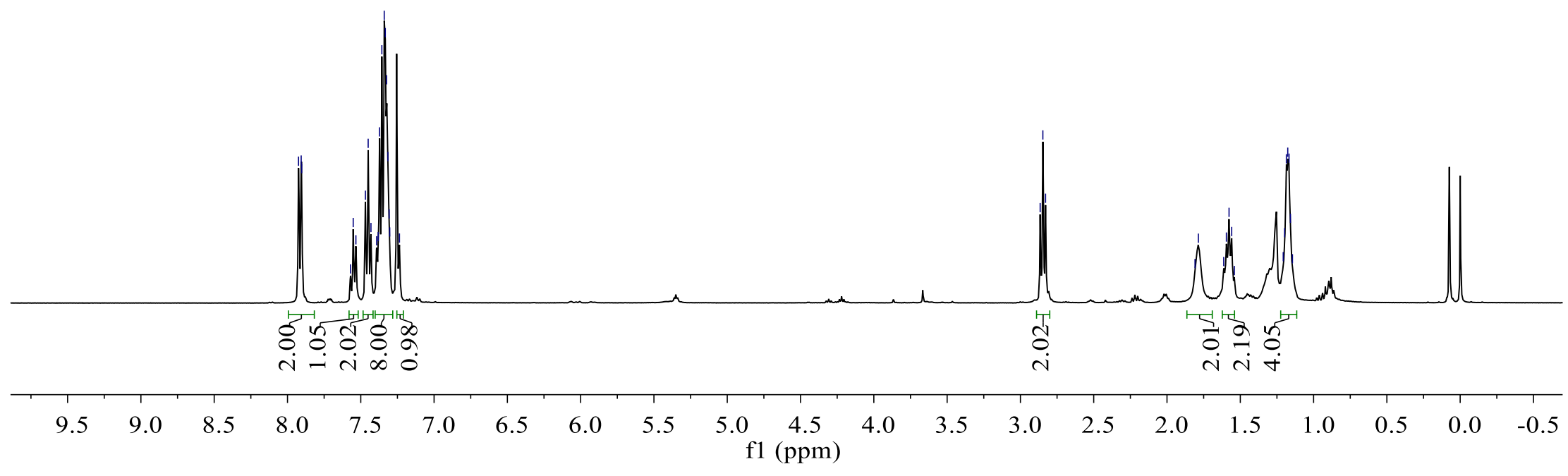

s9 


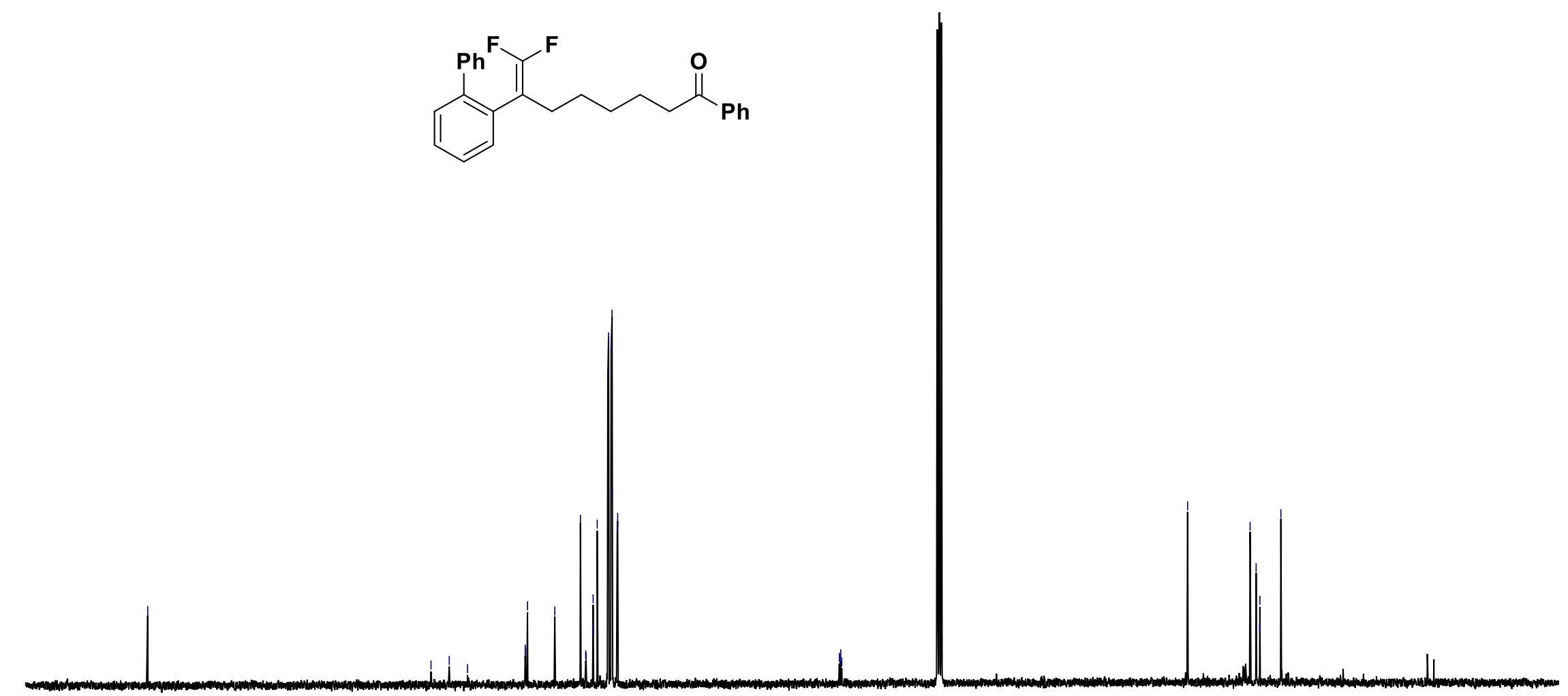


${ }^{19} \mathrm{~F}$ NMR-spectrum $\left(400 \mathrm{MHz}, \mathrm{CDCl}_{3}\right)$ of $\mathbf{3 c a}$

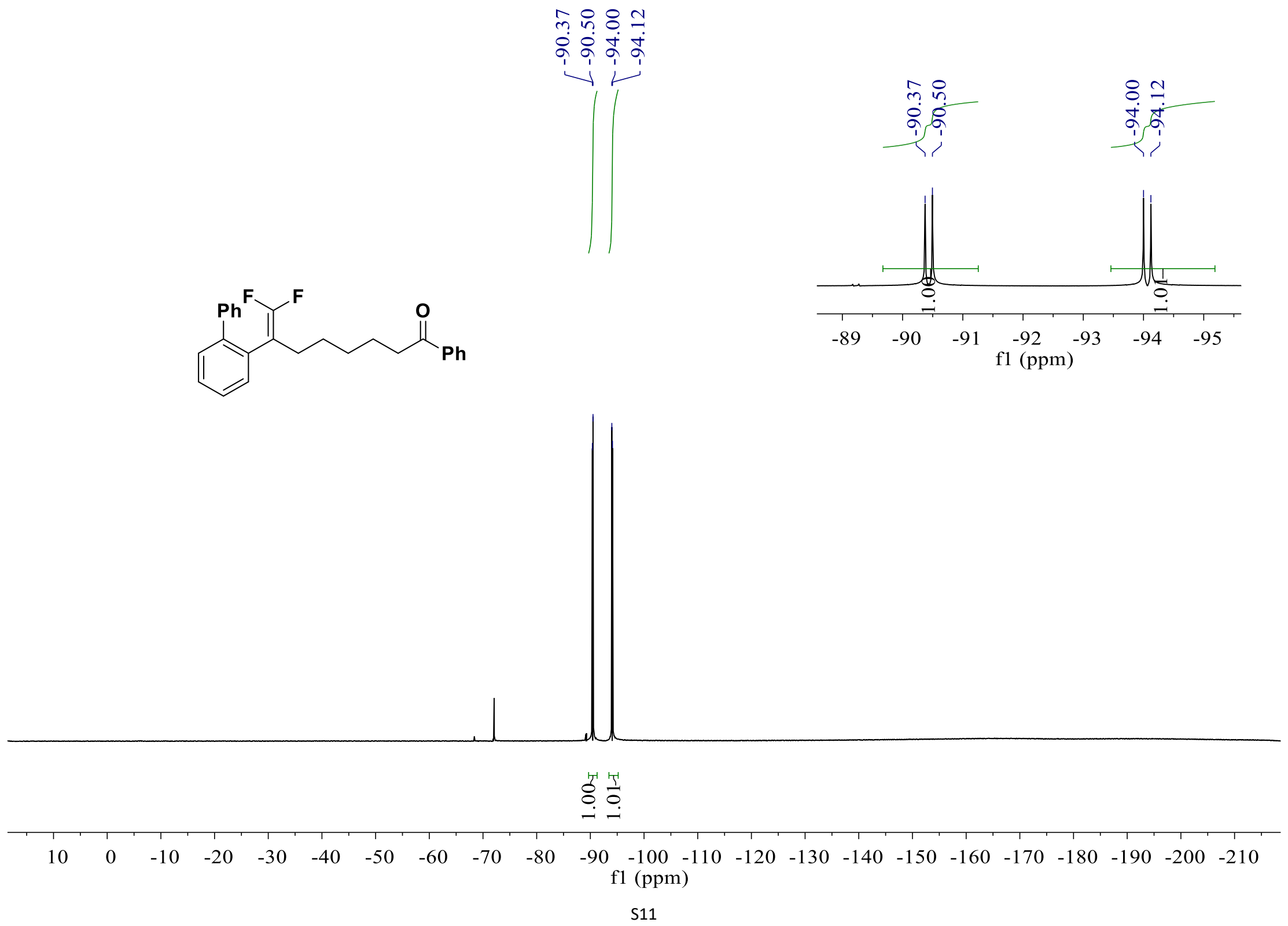



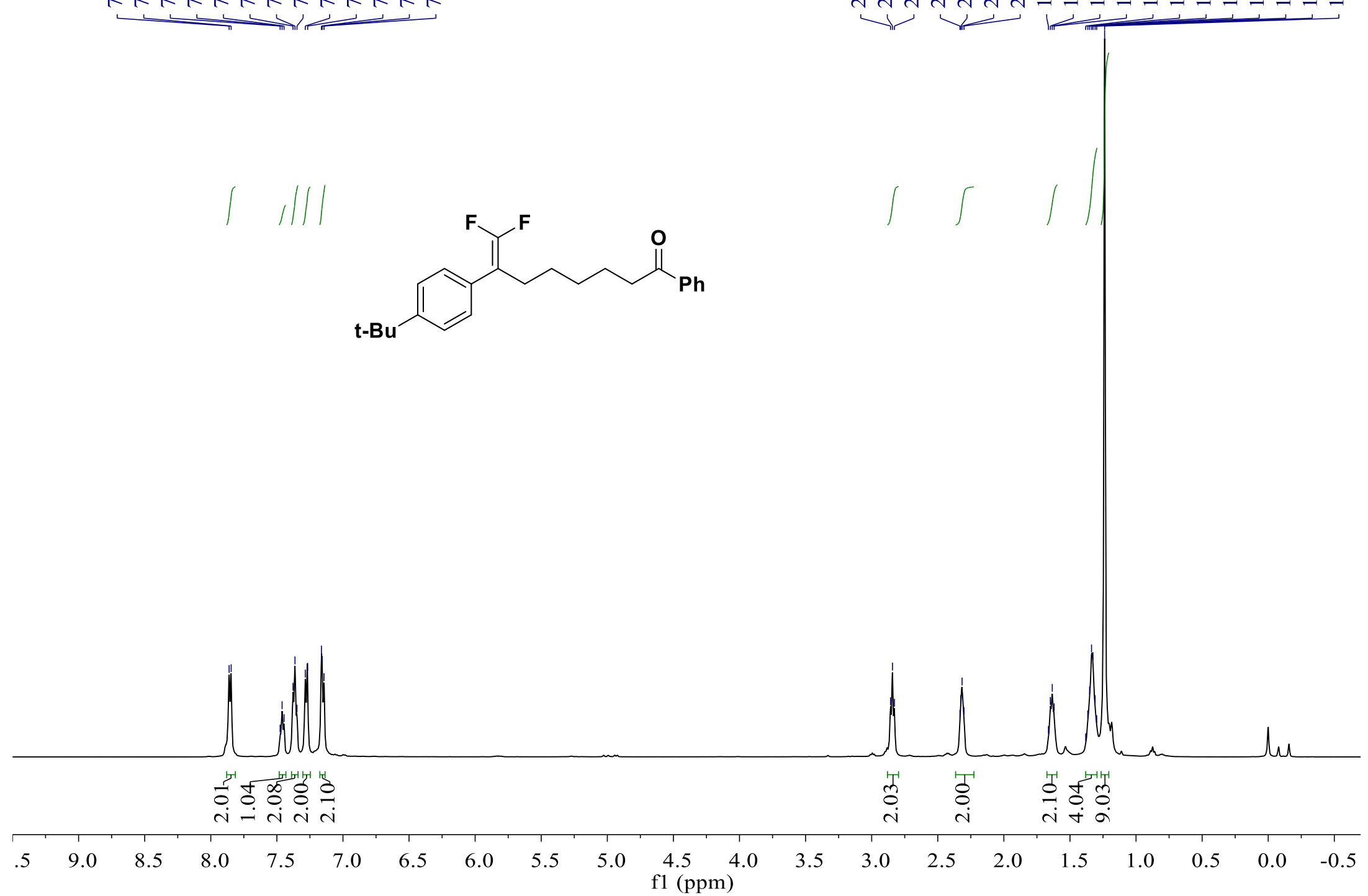
${ }^{13} \mathrm{C}\left\{{ }^{1} \mathrm{H}\right\}$ NMR-spectrum $\left(126 \mathrm{MHz}, \mathrm{CDCl}_{3}\right)$ of $\mathbf{3 d a}$

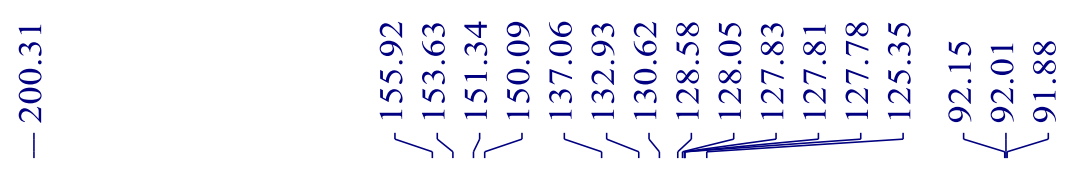

tin

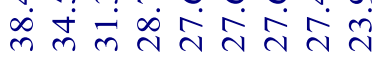
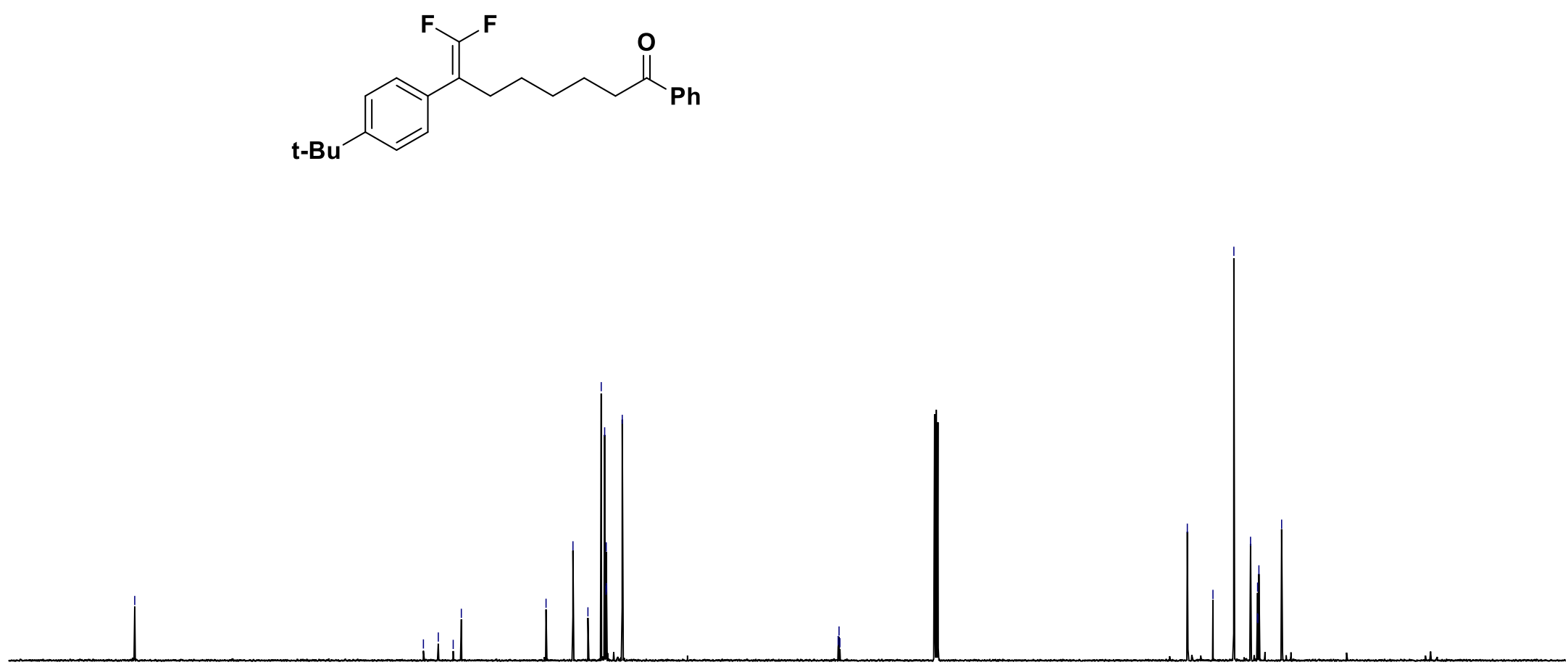

$\begin{array}{lllllllllllllllllllllll}210 & 200 & 190 & 180 & 170 & 160 & 150 & 140 & 130 & 120 & 110 \begin{array}{c}100 \\ \mathrm{f} 1(\mathrm{ppm})\end{array} & 90 & 80 & 70 & 60 & 50 & 40 & 30 & 20 & 10 & 0 & -10\end{array}$ S13 
${ }^{19}$ F NMR-spectrum (471 MHz, $\mathrm{CDCl}_{3}$ ) of $\mathbf{3 d a}$

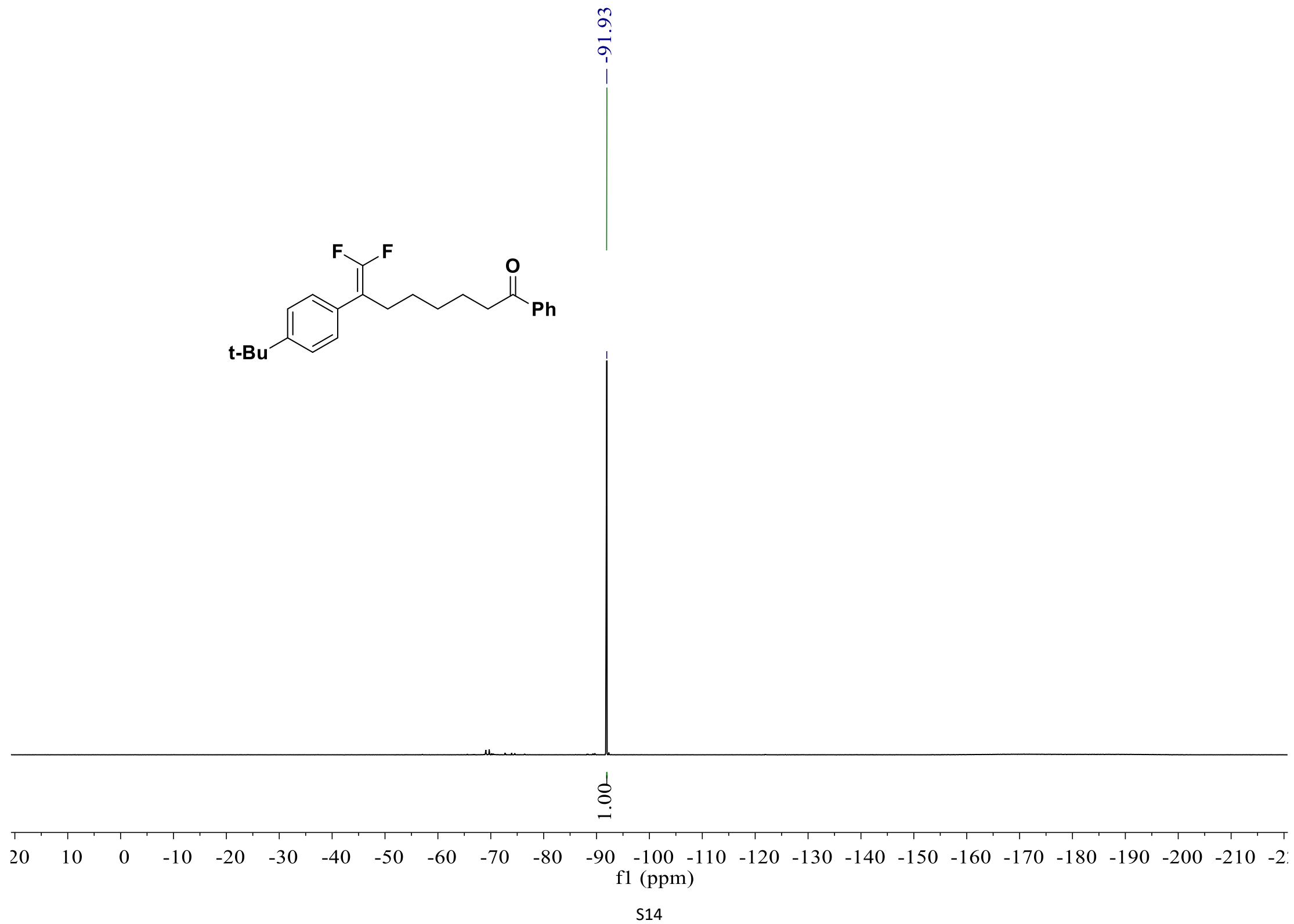




\section{${ }^{1} \mathrm{H}$ NMR-spectrum $\left(400 \mathrm{MHz}, \mathrm{CDCl}_{3}\right)$ of $\mathbf{3 e a}$}

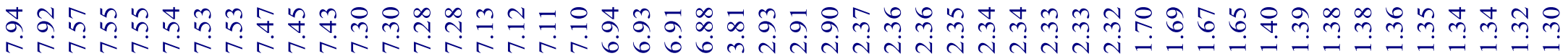

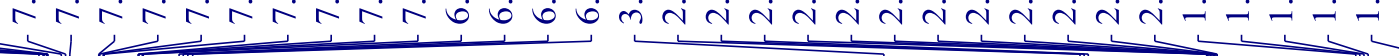

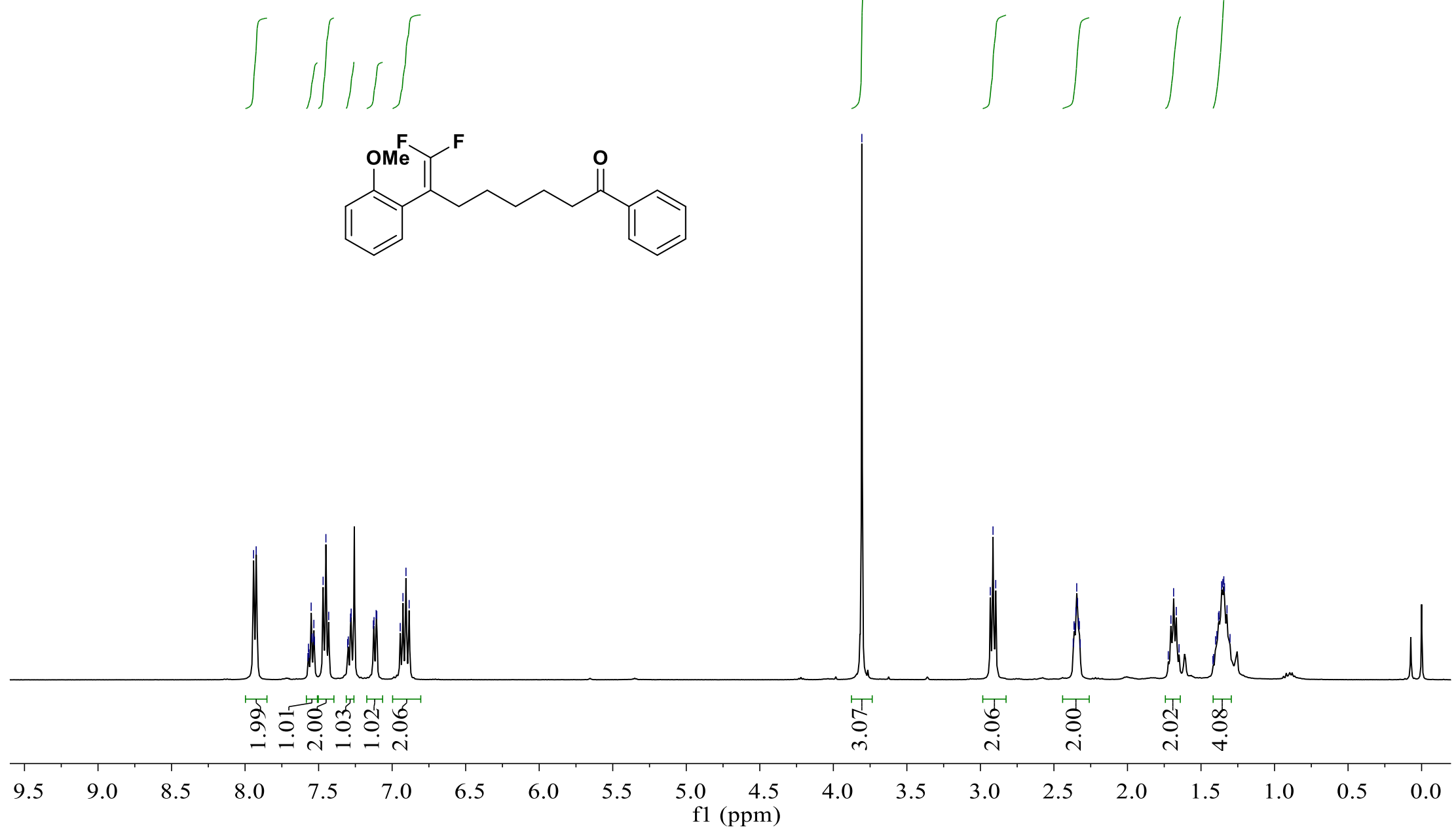

S15 
${ }^{13} \mathrm{C}\left\{{ }^{1} \mathrm{H}\right\}$ NMR-spectrum $\left(400 \mathrm{MHz}, \mathrm{CDCl}_{3}\right)$ of 3ea

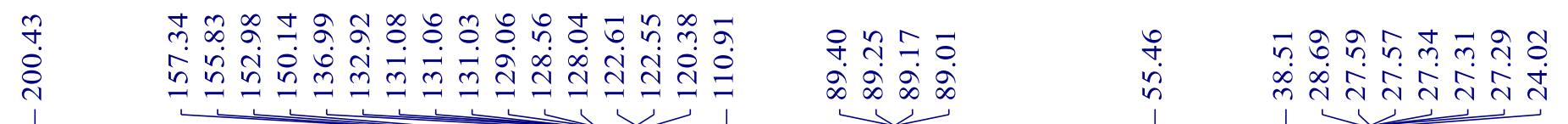
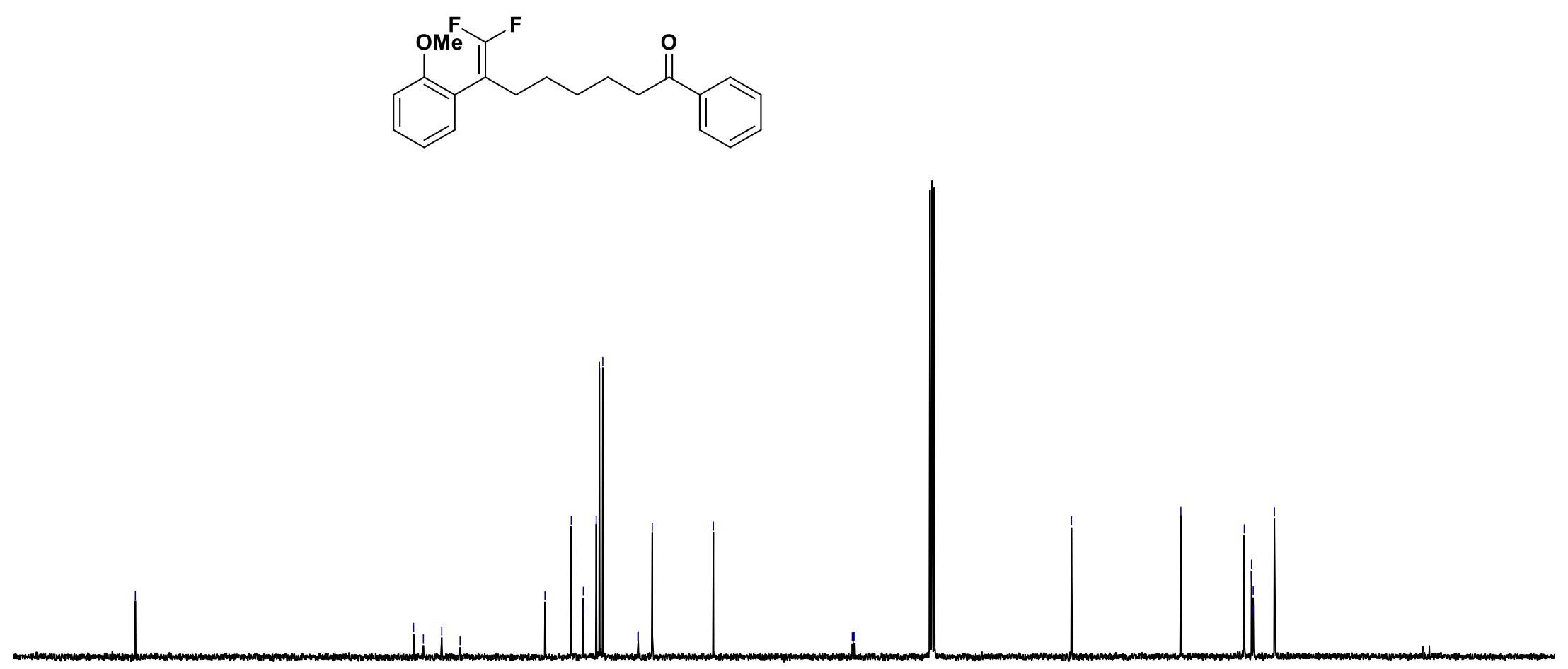

$\begin{array}{lllllllllllll}210 & 200 & 190 & 180 & 170 & 160 & 150 & 140 & 130 & 120 & 110 & 100 & 90\end{array}$

$60 \quad 50$

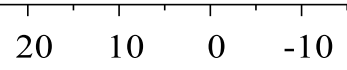

( 
${ }^{19} \mathrm{~F}$ NMR-spectrum $\left(376 \mathrm{MHz}, \mathrm{CDCl}_{3}\right)$ of $\mathbf{3 e a}$

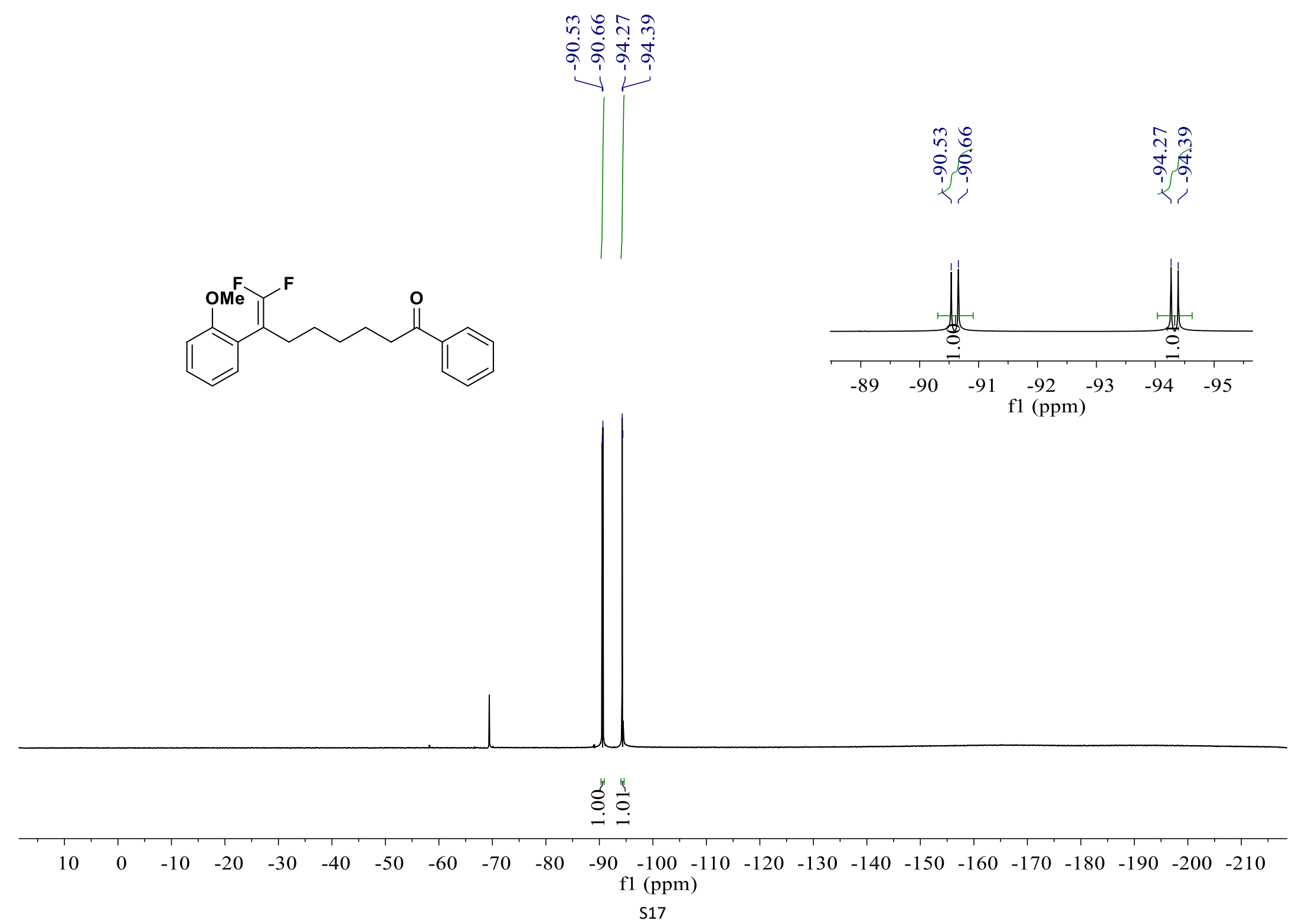




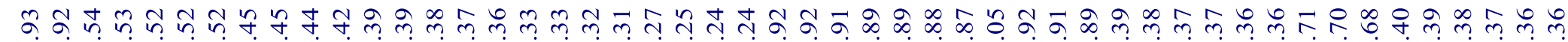

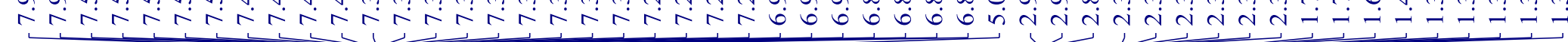

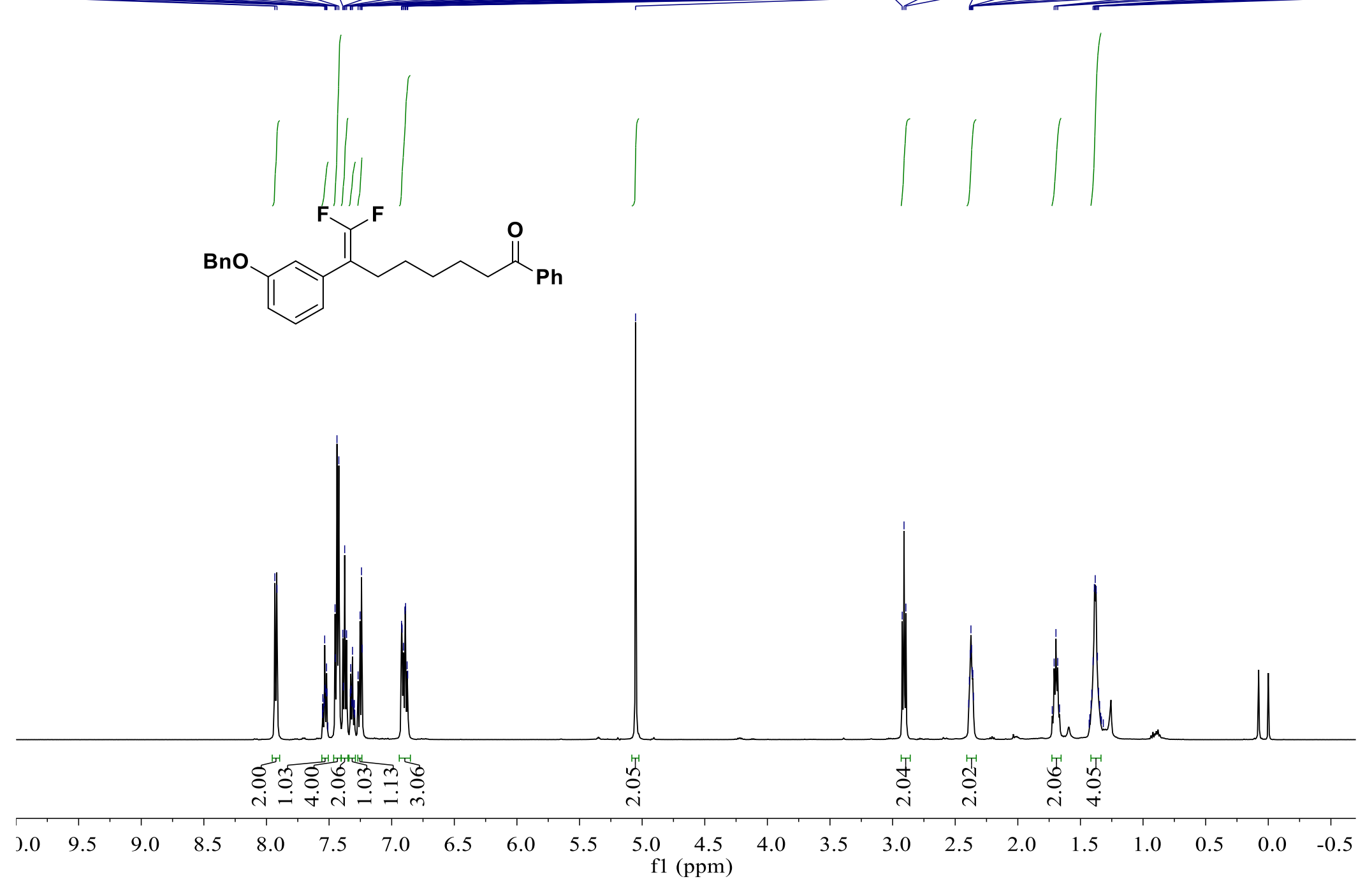

S18 
${ }^{13} \mathrm{C}\left\{{ }^{1} \mathrm{H}\right\}$ NMR-spectrum (126 MHz, $\left.\mathrm{CDCl}_{3}\right)$ of $\mathbf{3 f a}$

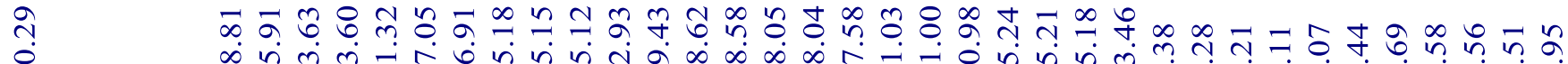

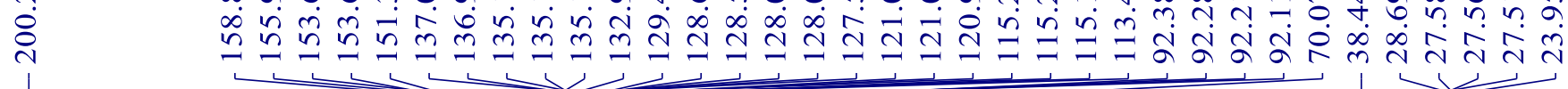
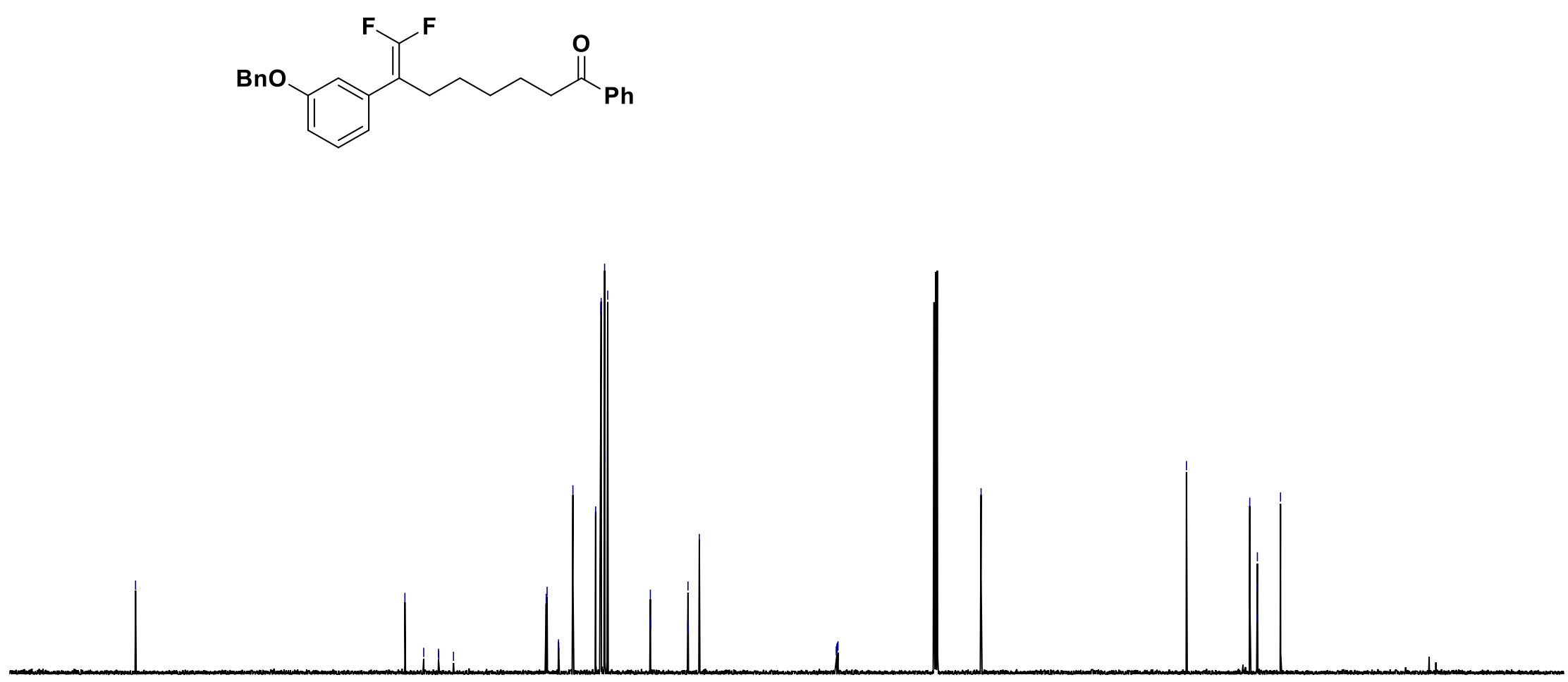

$\begin{array}{llllllllllll}210 & 200 & 190 & 180 & 170 & 160 & 150 & 140 & 130 & 120 & 110 & 100\end{array}$

f1 (ppm)

s19 
${ }^{19} \mathrm{~F}$ NMR-spectrum $\left(471 \mathrm{MHz}, \mathrm{CDCl}_{3}\right)$ of $\mathbf{3 f a}$

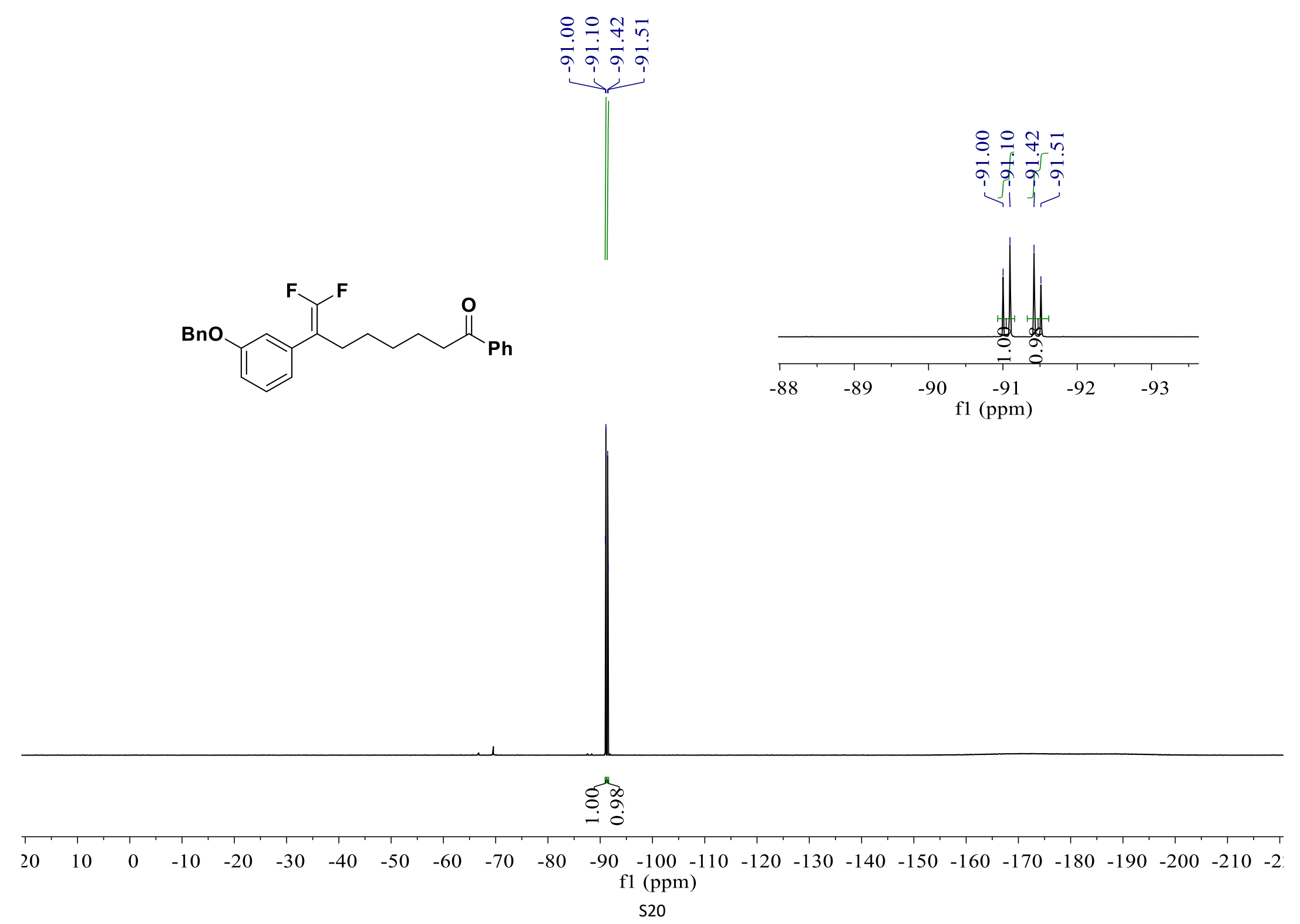


${ }^{1} \mathrm{H}$ NMR-spectrum $\left(500 \mathrm{MHz}, \mathrm{CDCl}_{3}\right)$ of $\mathbf{3 g a}$

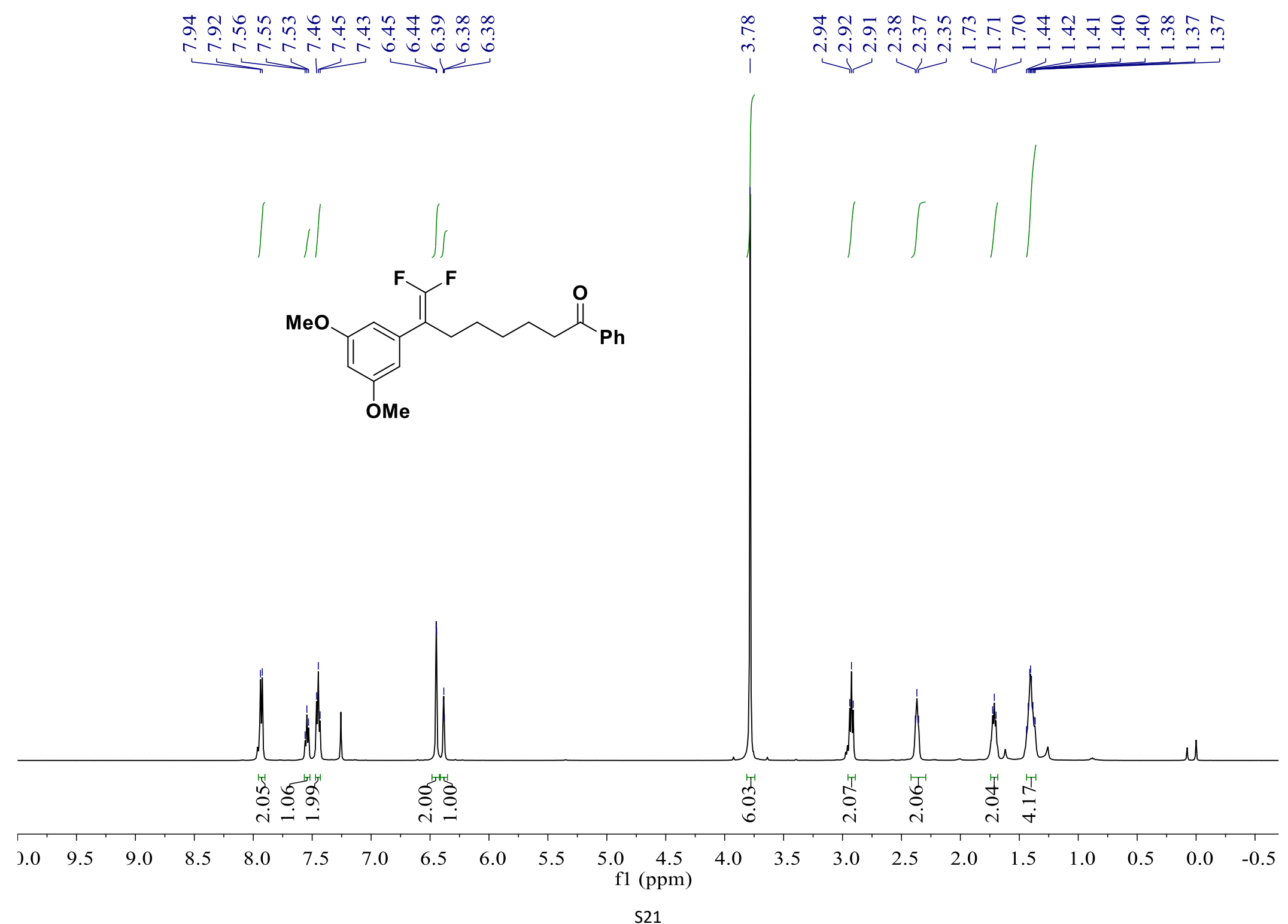


${ }^{13} \mathrm{C}\left\{{ }^{1} \mathrm{H}\right\}$ NMR-spectrum $\left(126 \mathrm{MHz}, \mathrm{CDCl}_{3}\right)$ of 3ga

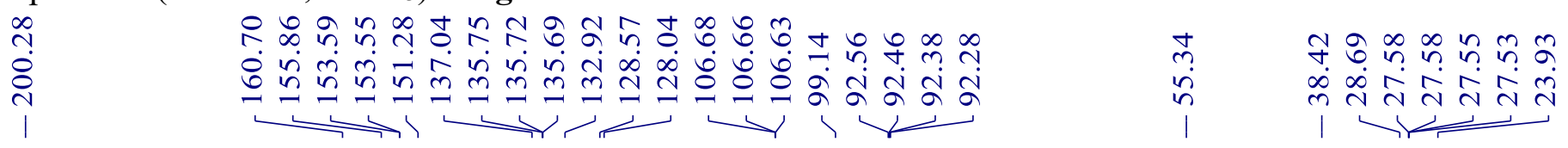
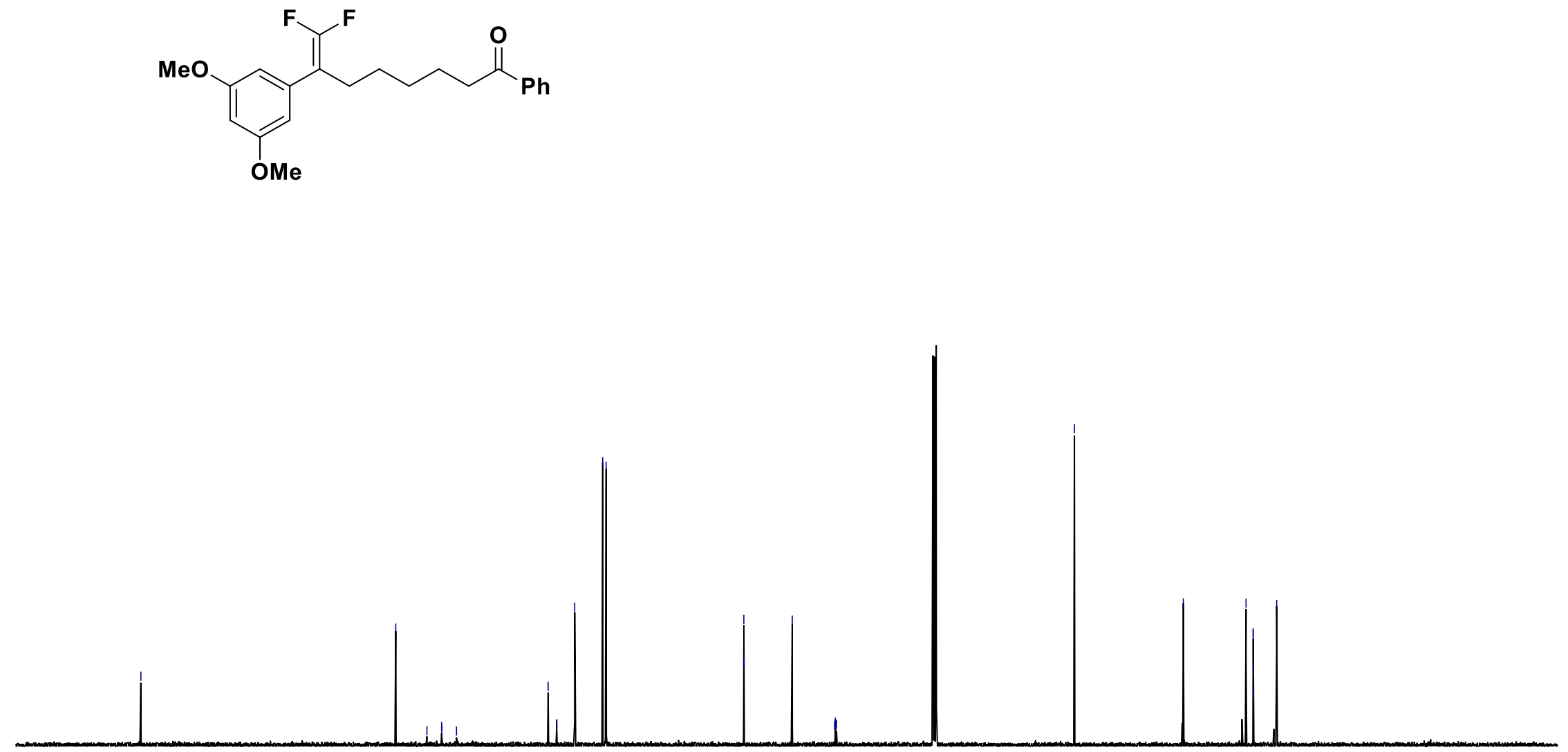

$\begin{array}{llllllllllllllllllllllll}210 & 200 & 190 & 180 & 170 & 160 & 150 & 140 & 130 & 120 & \begin{array}{c}110 \\ \mathrm{f} 1 \mathrm{100}(\mathrm{ppm})\end{array} & 90 & 80 & 70 & 60 & 50 & 40 & 30 & 20 & 10 & 0 & -10\end{array}$ S22 
${ }^{19} \mathrm{~F}$ NMR-spectrum (371 MHz, $\mathrm{CDCl}_{3}$ ) of 3ga

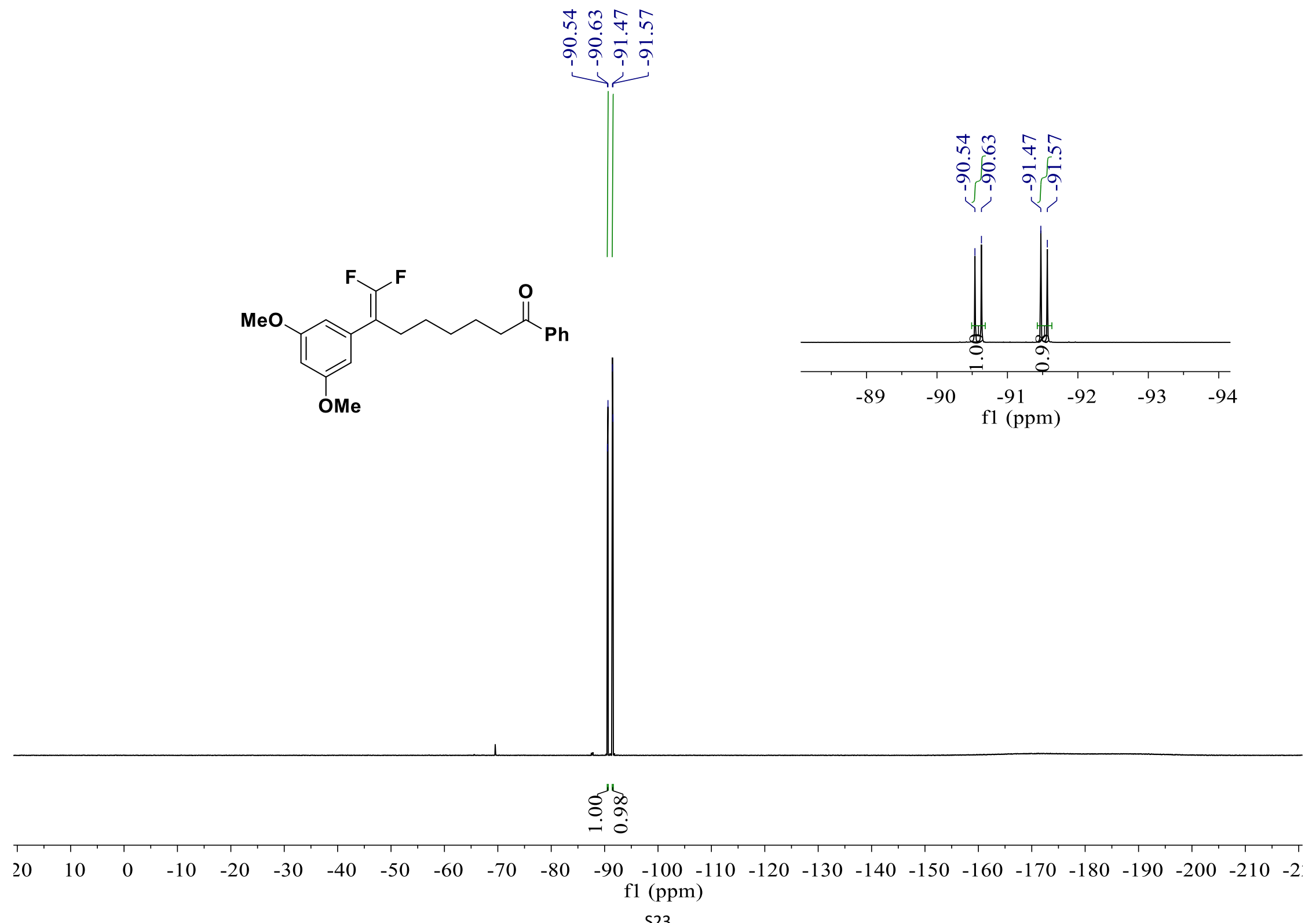

S23 

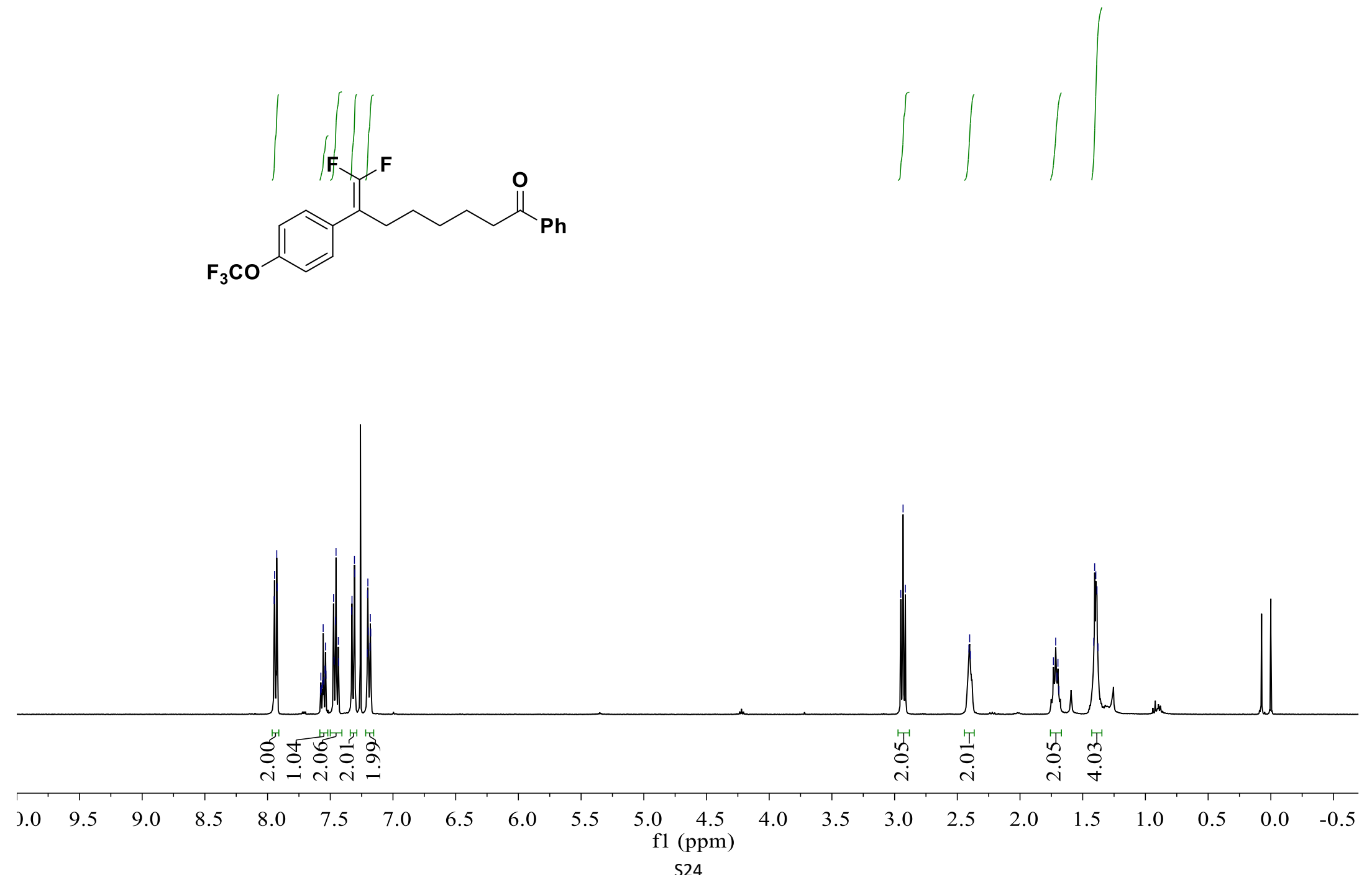
${ }^{13} \mathrm{C}\left\{{ }^{1} \mathrm{H}\right\}$ NMR-spectrum $\left(400 \mathrm{MHz}, \mathrm{CDCl}_{3}\right)$ of $\mathbf{3 h a}$
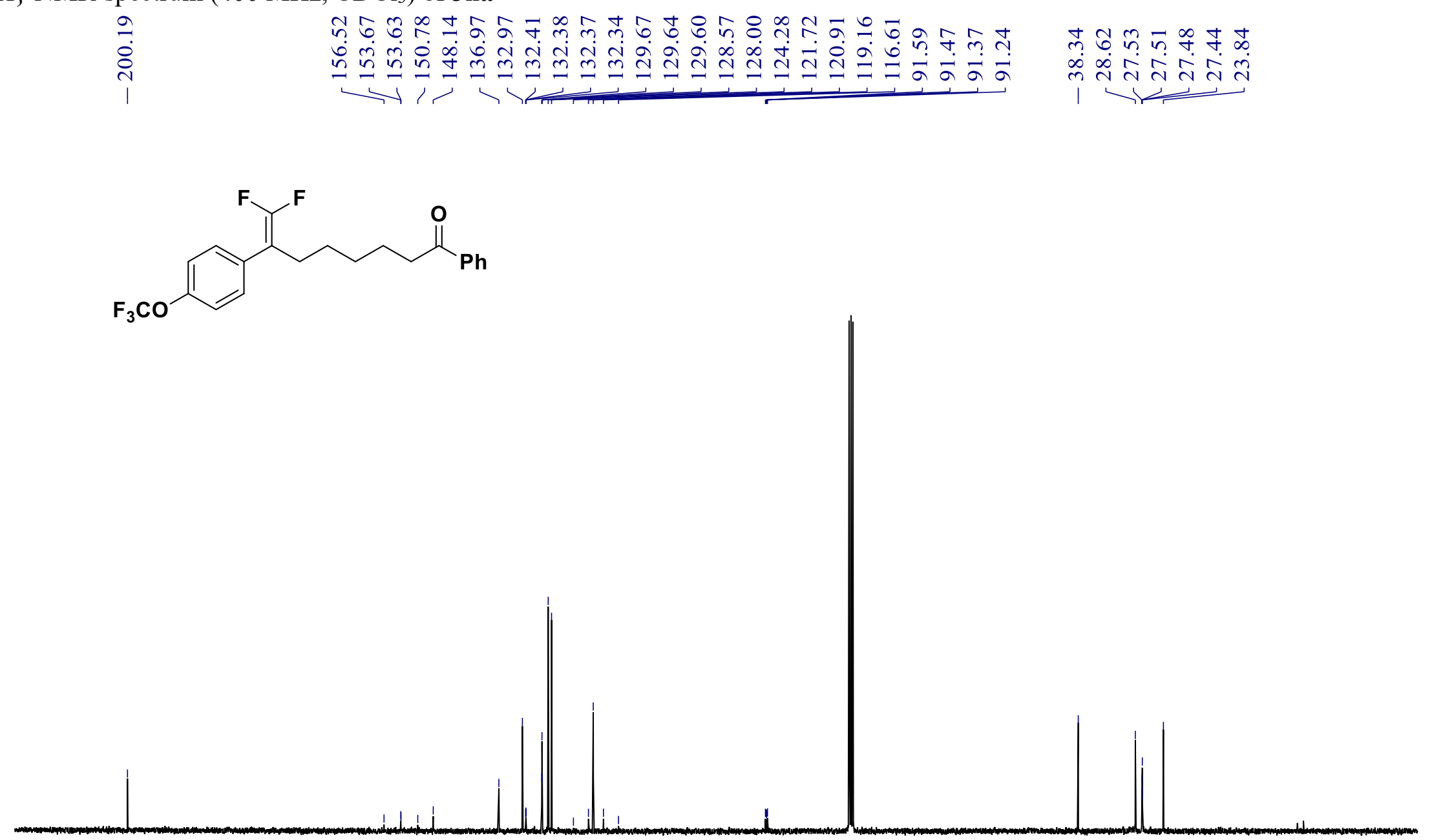

$\begin{array}{llllllllllll}210 & 200 & 190 & 180 & 170 & 160 & 150 & 140 & 130 & 120 & 110 & 100 \\ \mathrm{f} 1(\mathrm{ppm})\end{array}$ 
${ }^{19} \mathrm{~F}$ NMR-spectrum $\left(101 \mathrm{MHz}, \mathrm{CDCl}_{3}\right)$ of $\mathbf{3 h a}$

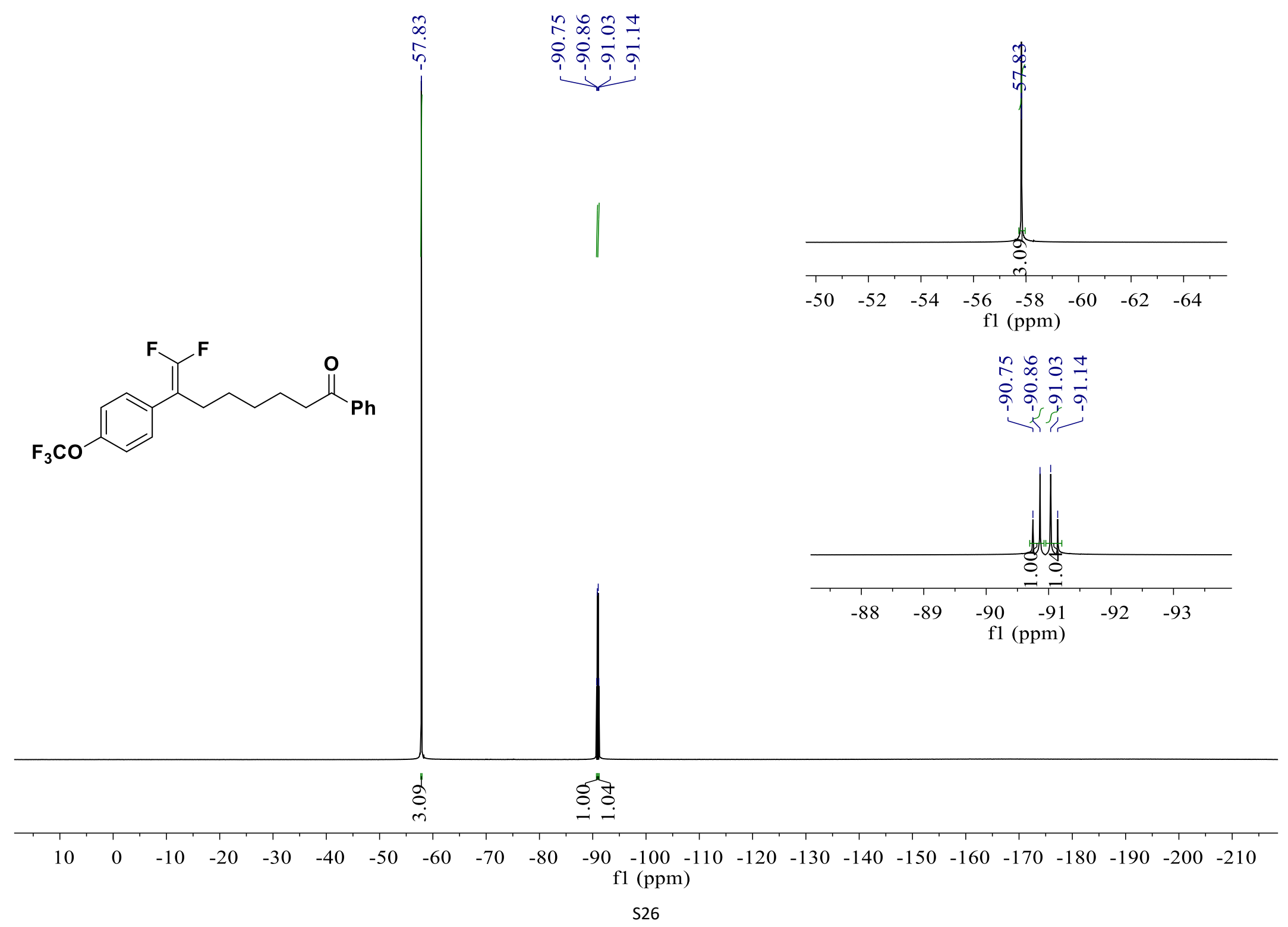



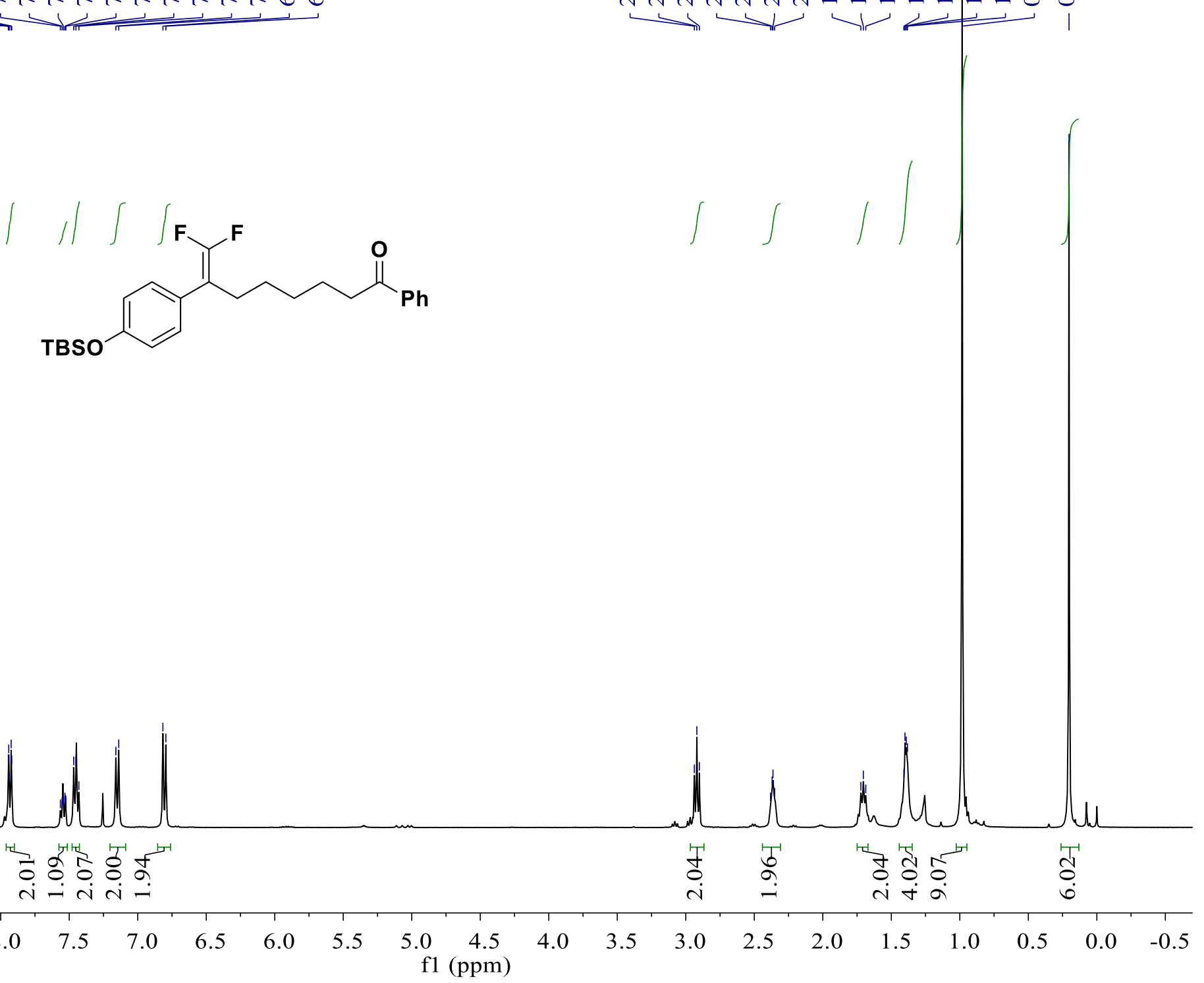
${ }^{13} \mathrm{C}\left\{{ }^{1} \mathrm{H}\right\}$ NMR-spectrum $\left(400 \mathrm{MHz}, \mathrm{CDCl}_{3}\right)$ of 3ia

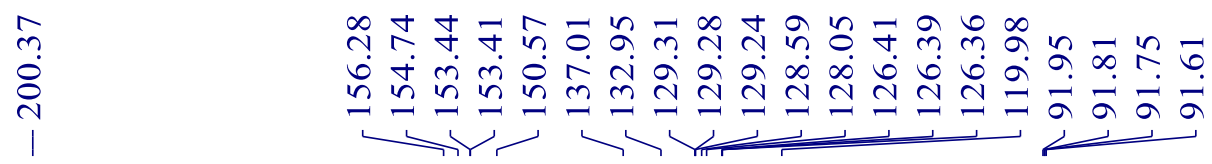

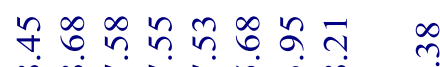

i
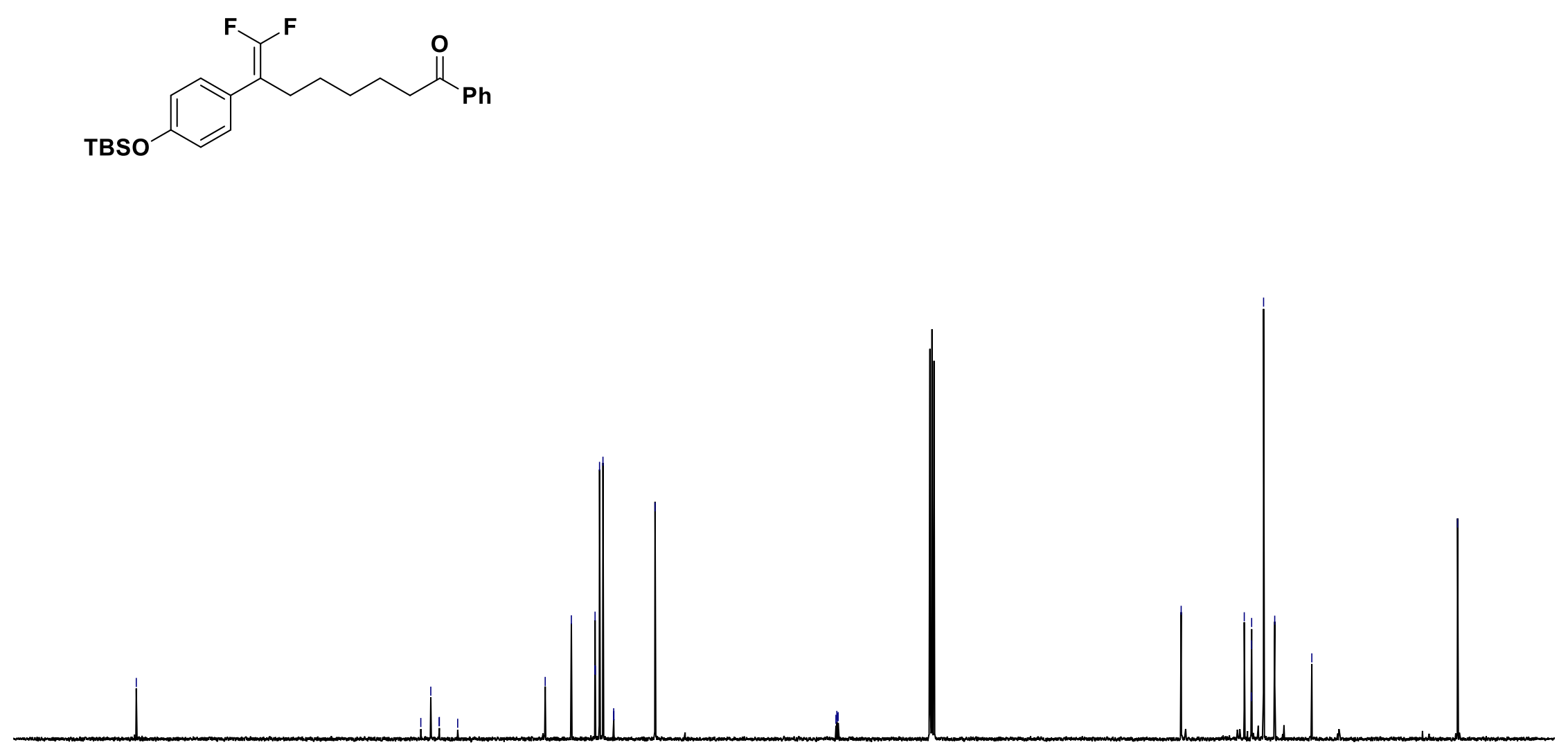

$\begin{array}{lllllllllllllllllllllll}210 & 200 & 190 & 180 & 170 & 160 & 150 & 140 & 130 & 120 & 110 \begin{array}{c}100 \\ \mathrm{f} 1(\mathrm{ppm})\end{array} & 90 & 80 & 70 & 60 & 50 & 40 & 30 & 20 & 10 & 0 & -10\end{array}$ S28 
${ }^{19} \mathrm{~F}$ NMR-spectrum $\left(376 \mathrm{MHz}, \mathrm{CDCl}_{3}\right)$ of 3 ia

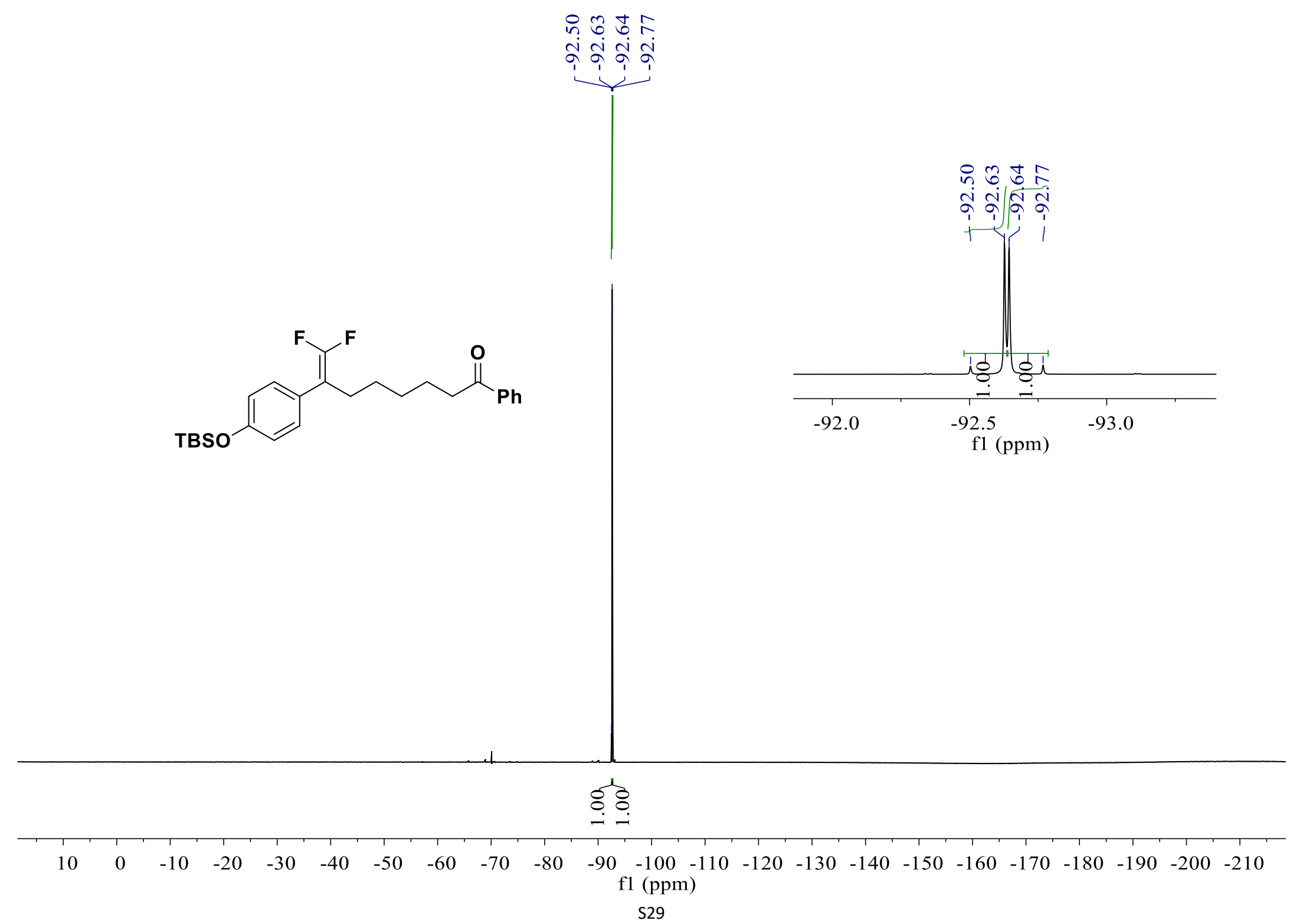


${ }^{1} \mathrm{H}$ NMR-spectrum $\left(500 \mathrm{MHz}, \mathrm{CDCl}_{3}\right)$ of $\mathbf{3 j a}$

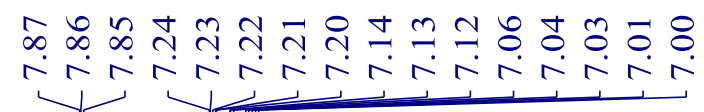

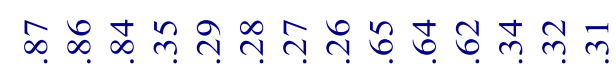

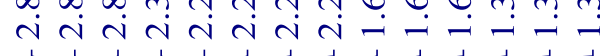

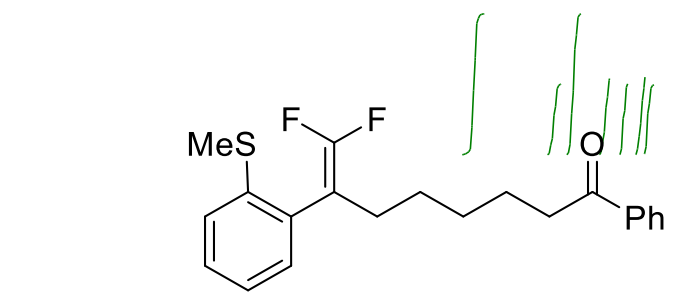

$\longrightarrow$ ill
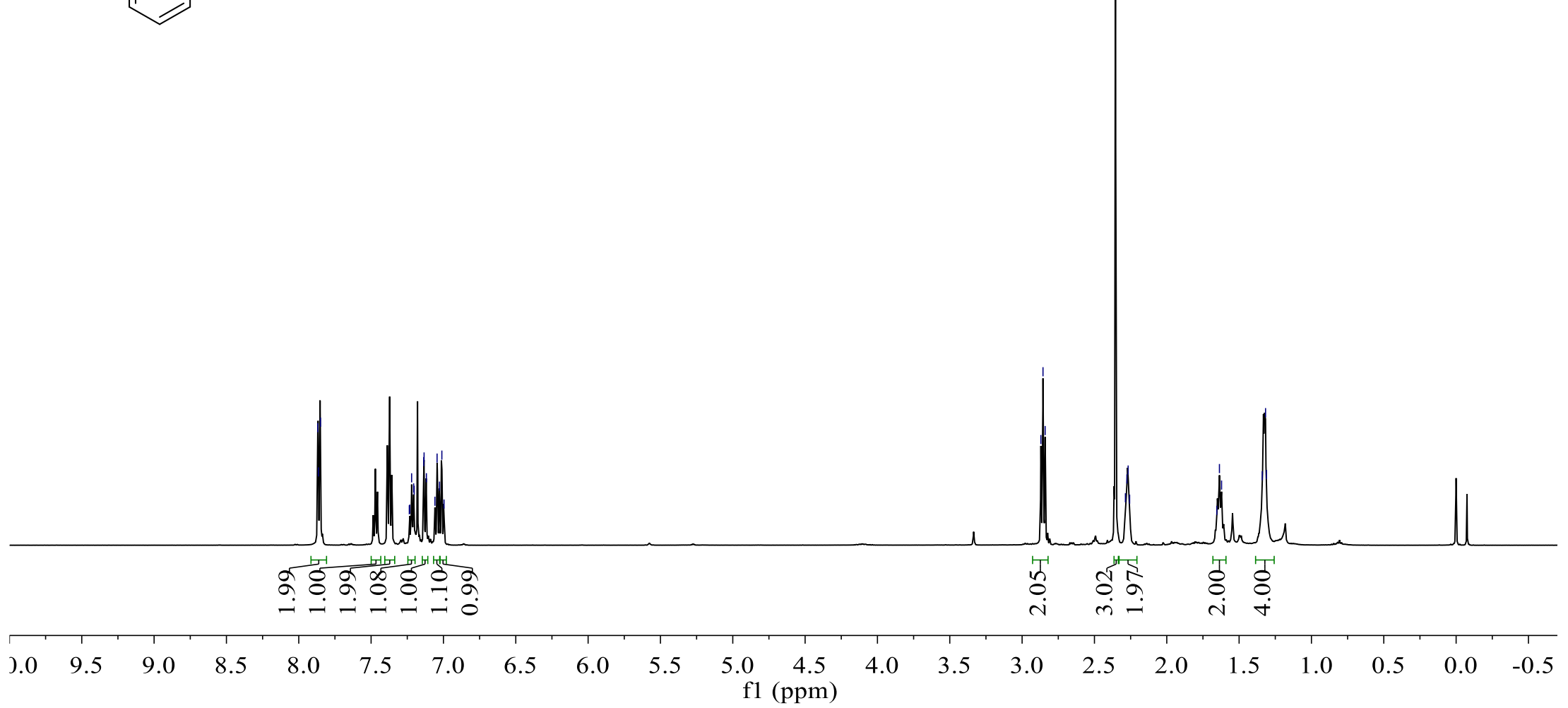

S30 
${ }^{13} \mathrm{C}\left\{{ }^{1} \mathrm{H}\right\}$ NMR-spectrum $\left(126 \mathrm{MHz}, \mathrm{CDCl}_{3}\right)$ of $\mathbf{3 j a}$

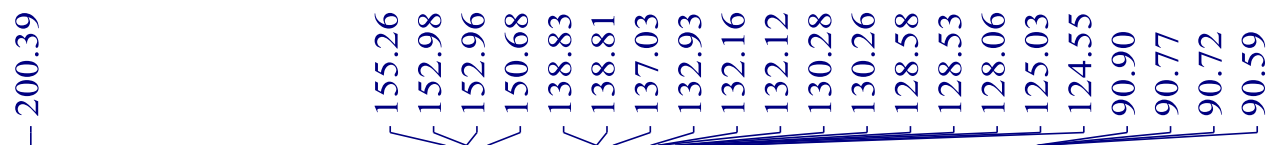

守

ind
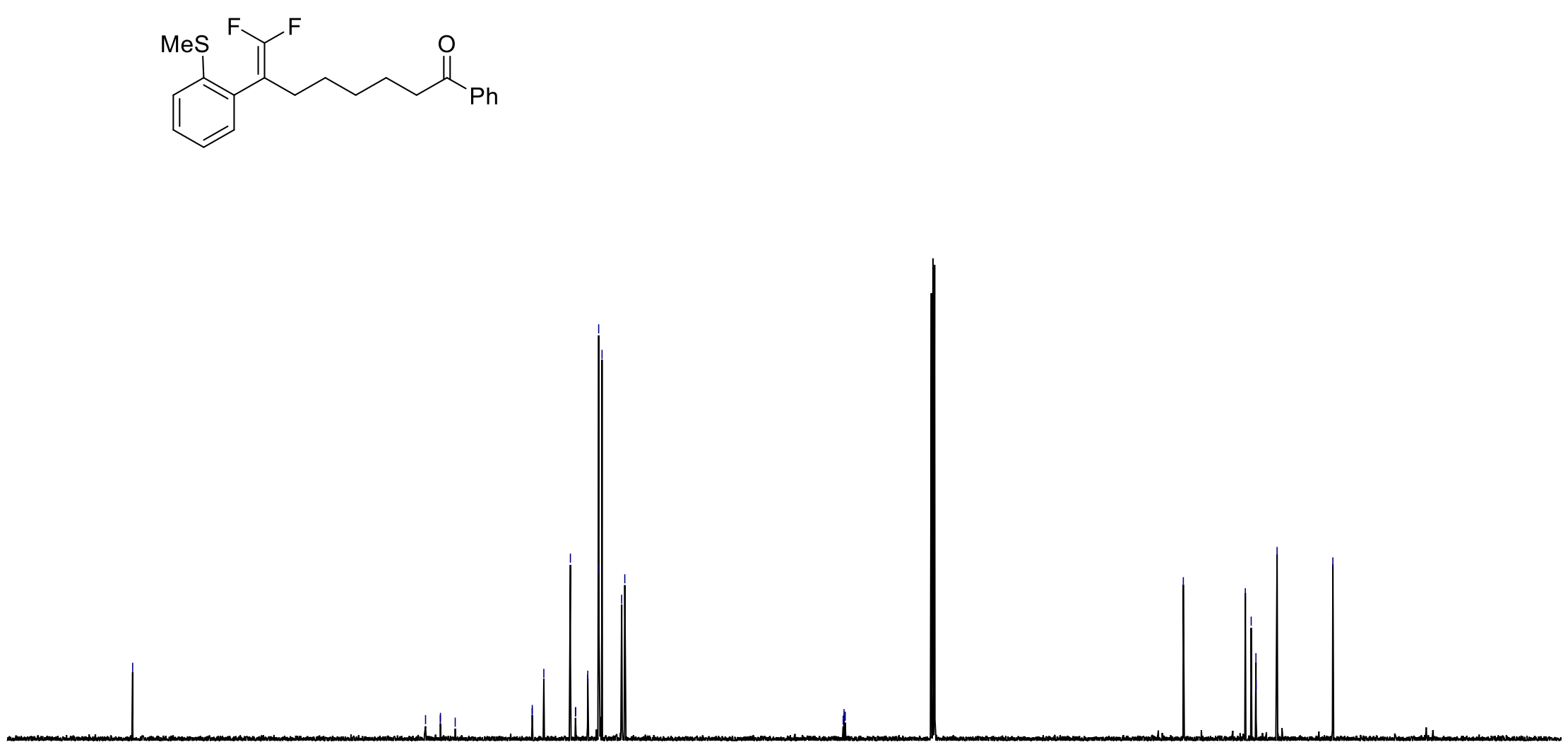

$\begin{array}{lllllllllllllllllllllllllll}210 & 200 & 190 & 180 & 170 & 160 & 150 & 140 & 130 & 120 & 110 & 100 & 90 & 80 & 70 & 60 & 50 & 40 & 30 & 20 & 10 & 0 & -10 & 0\end{array}$

f1 (ppm)

S31 
${ }^{19}$ F NMR-spectrum $\left(471 \mathrm{MHz}, \mathrm{CDCl}_{3}\right)$ of $\mathbf{3 j a}$

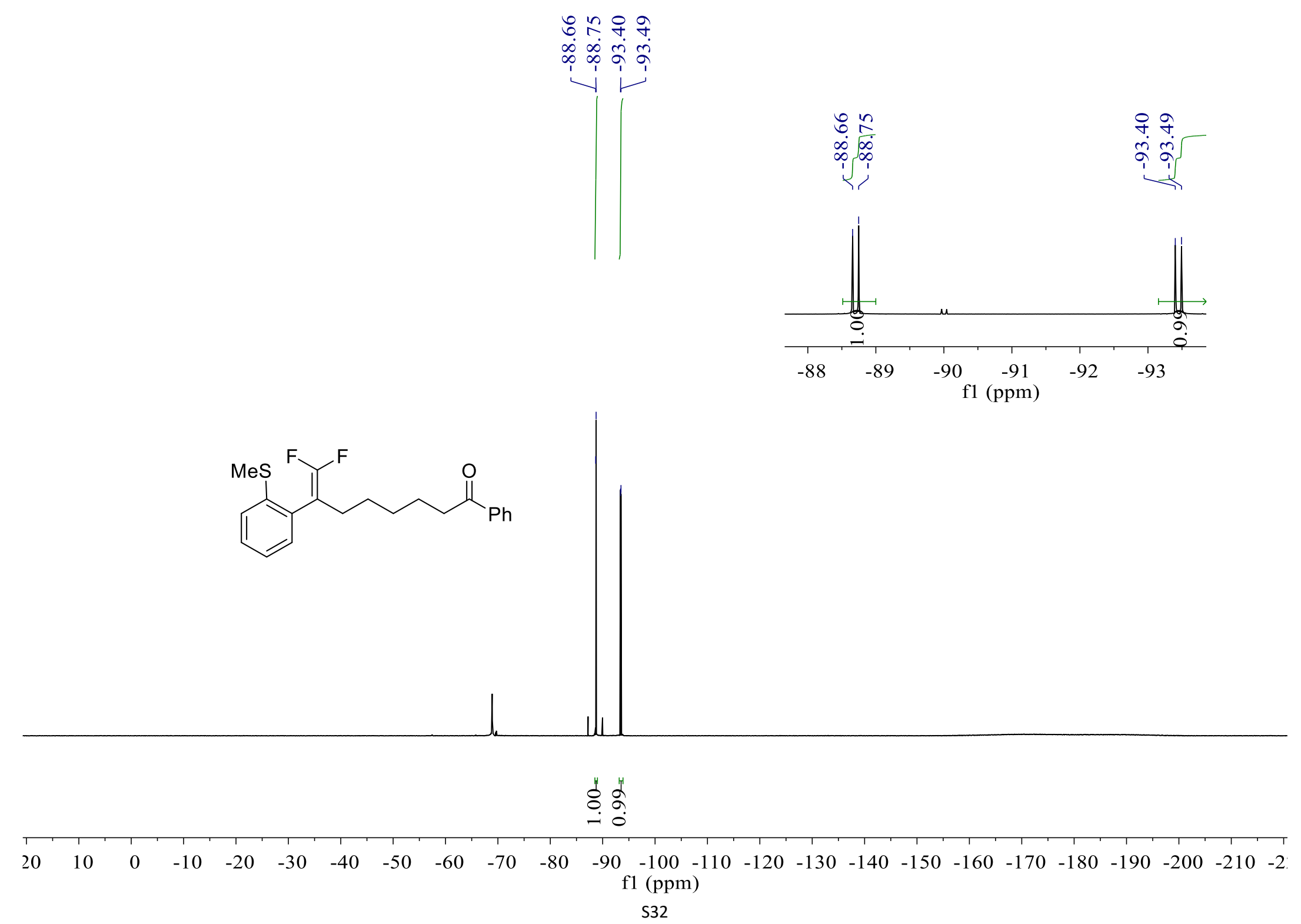




\section{${ }^{1} \mathrm{H}$ NMR-spectrum $\left(400 \mathrm{MHz}, \mathrm{CDCl}_{3}\right)$ of $\mathbf{3 k a}$}

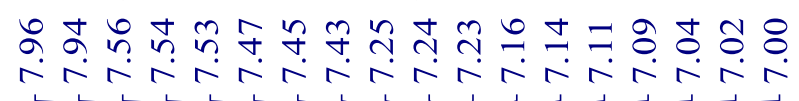

ลํำ

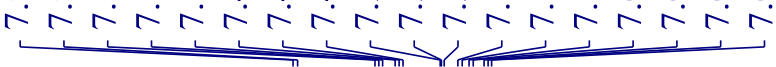

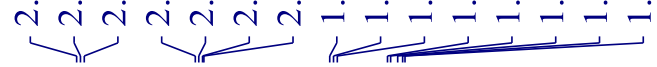
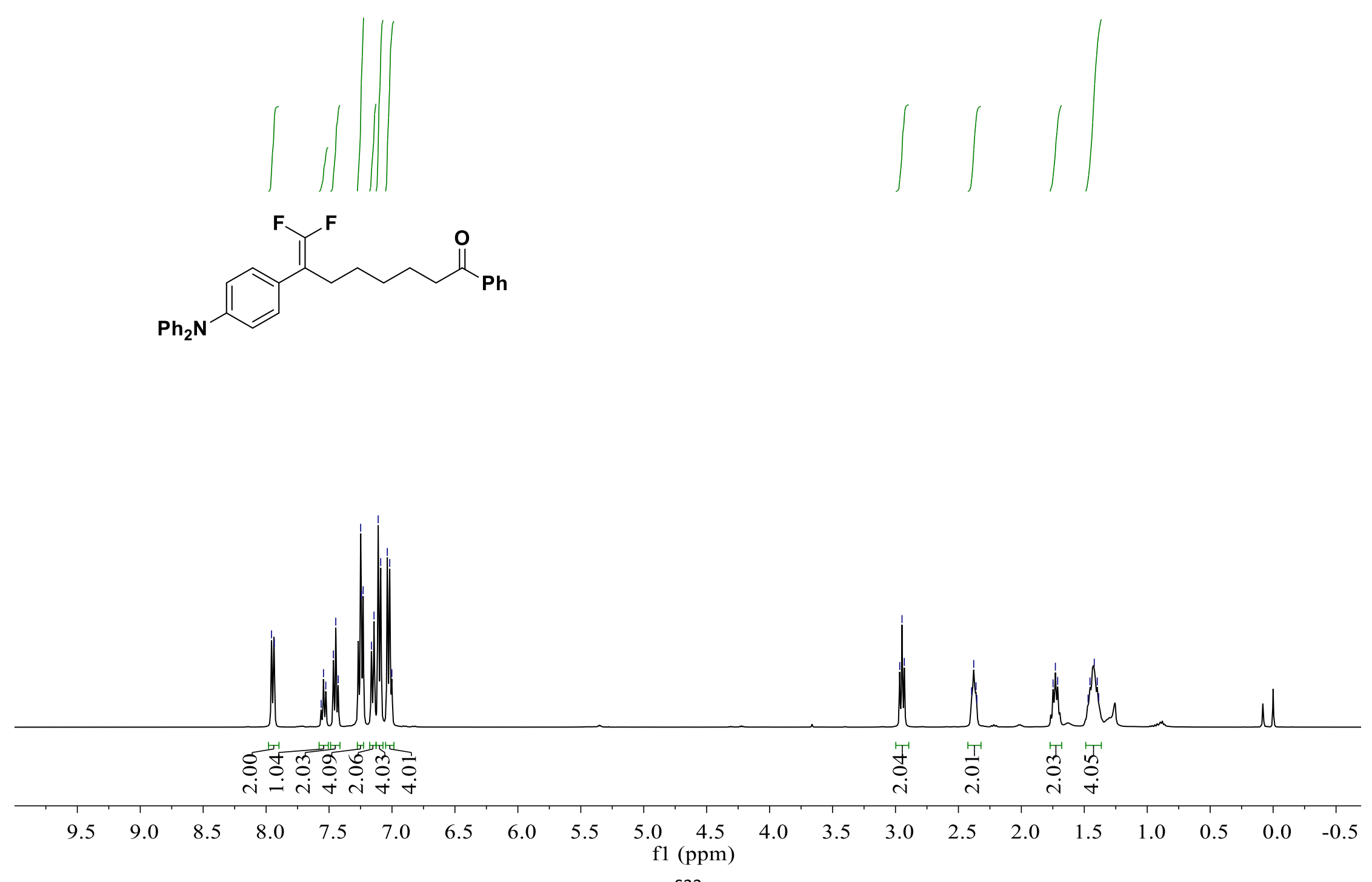

S33 
${ }^{13} \mathrm{C}\left\{{ }^{1} \mathrm{H}\right\}$ NMR-spectrum $\left(101 \mathrm{MHz}, \mathrm{CDCl}_{3}\right)$ of $\mathbf{3 k a}$

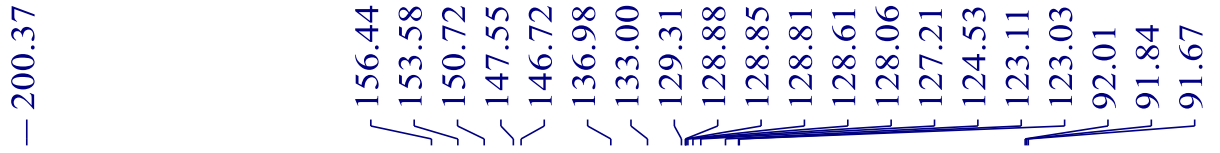

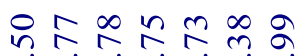

m

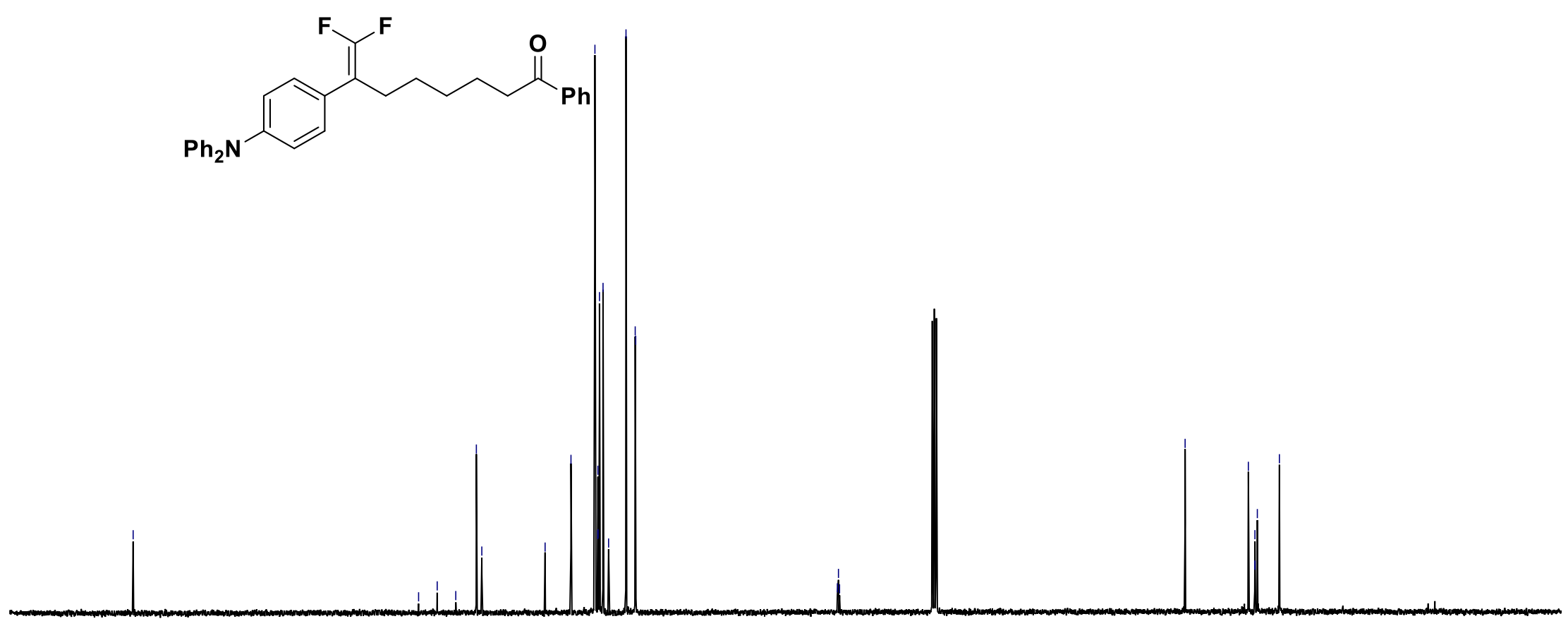

$150 \quad 140$

$\begin{array}{ll}0 \quad 130 & 120\end{array}$

20110

$0 \quad 100$
1100

8070

60

50

$1(\mathrm{pp}$

S34 
${ }^{19} \mathrm{~F}$ NMR-spectrum $\left(376 \mathrm{MHz}, \mathrm{CDCl}_{3}\right)$ of $3 \mathbf{k a}$

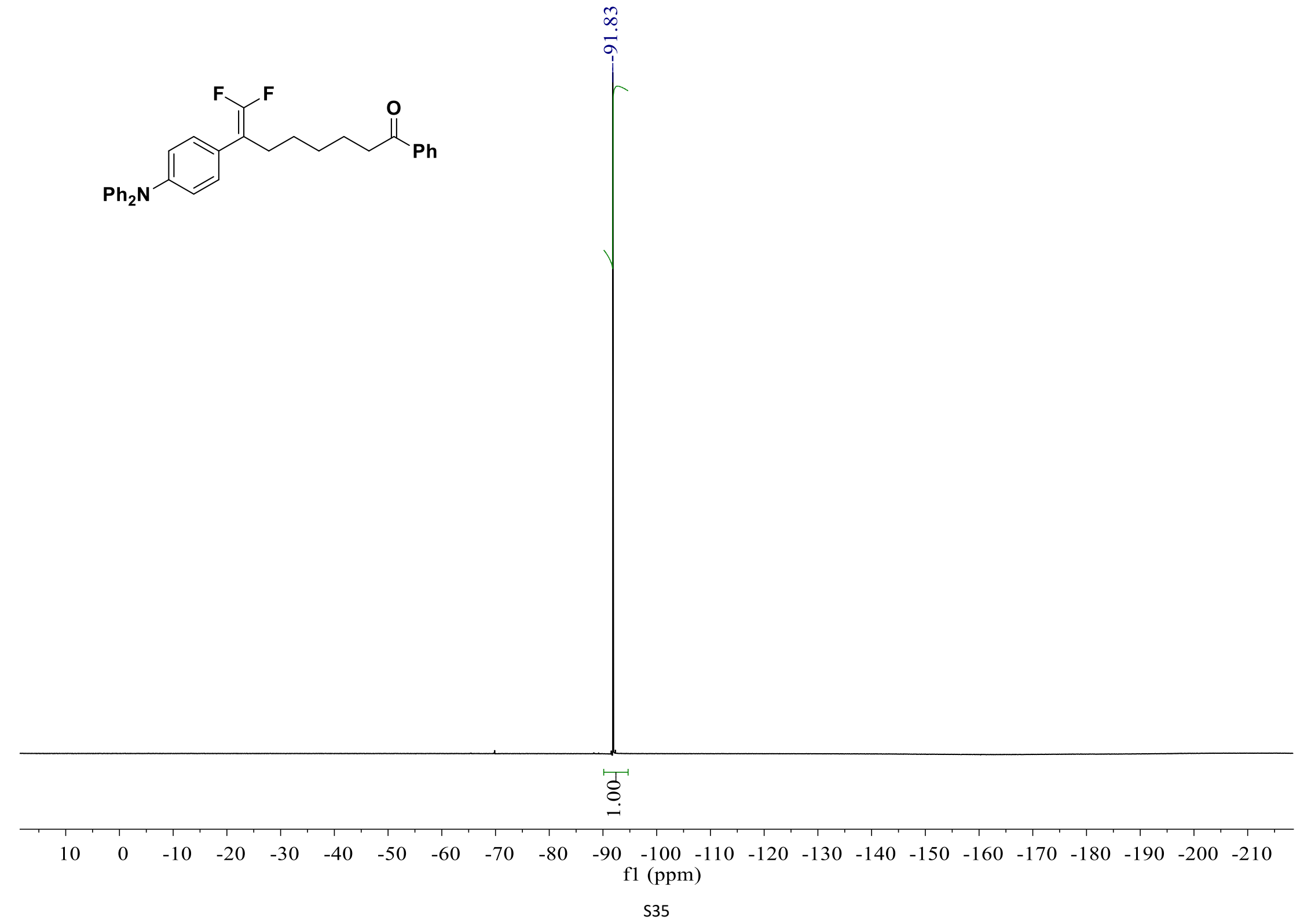


${ }^{1} \mathrm{H}$ NMR-spectrum $\left(500 \mathrm{MHz}, \mathrm{CDCl}_{3}\right)$ of $3 \mathbf{l a}$

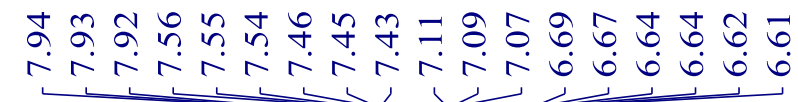

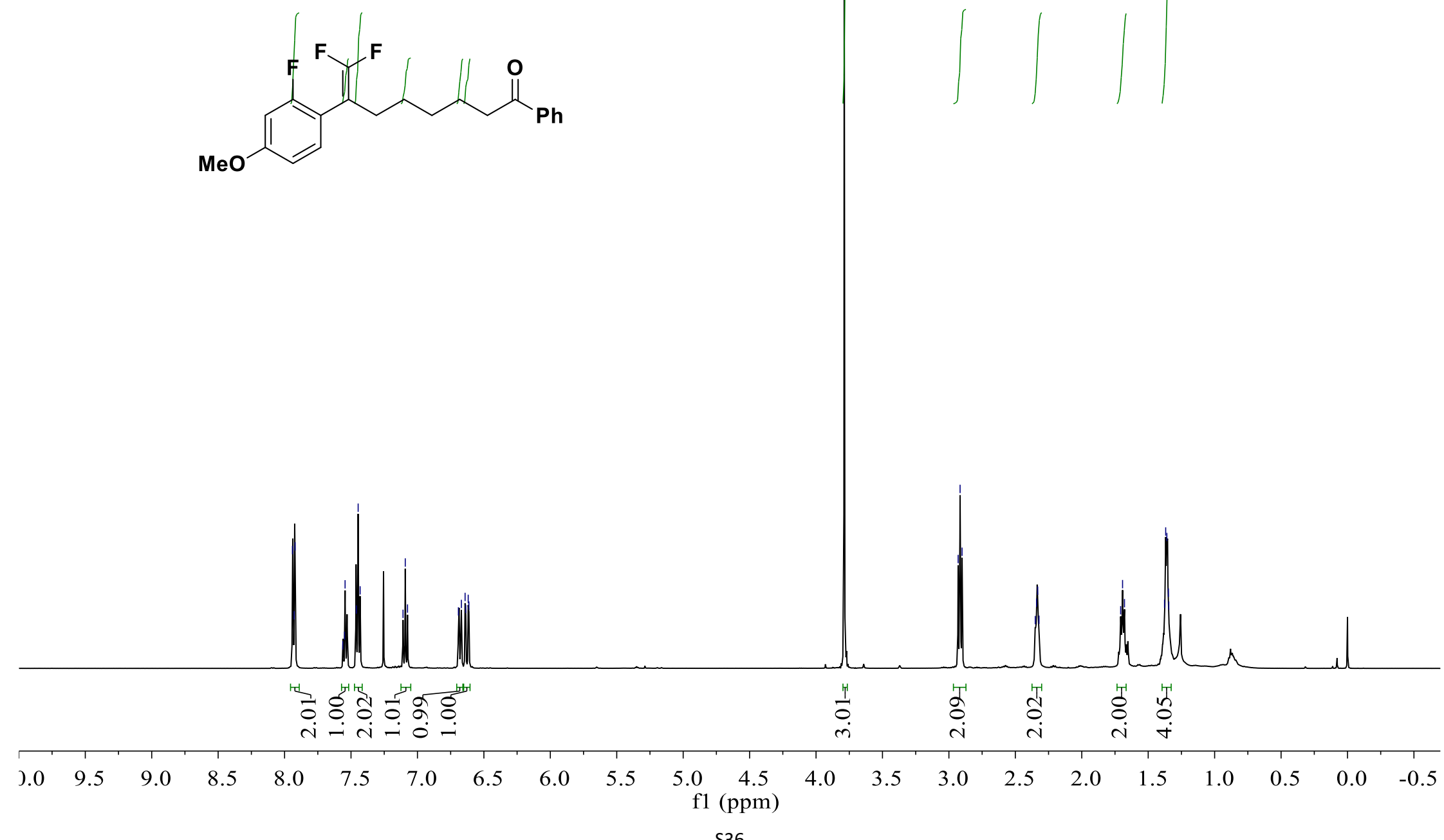

શ.

is 4 i n

$\longrightarrow$ L

$\$ 36$ 
${ }^{13} \mathrm{C}\left\{{ }^{1} \mathrm{H}\right\}$ NMR-spectrum (126 MHz, $\left.\mathrm{CDCl}_{3}\right)$ of 3la

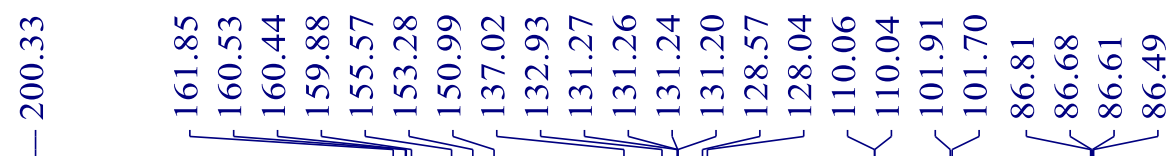

n) $\quad$ f.

in $\quad \infty \quad \infty i \vec{i} \hat{\sim}$

i.
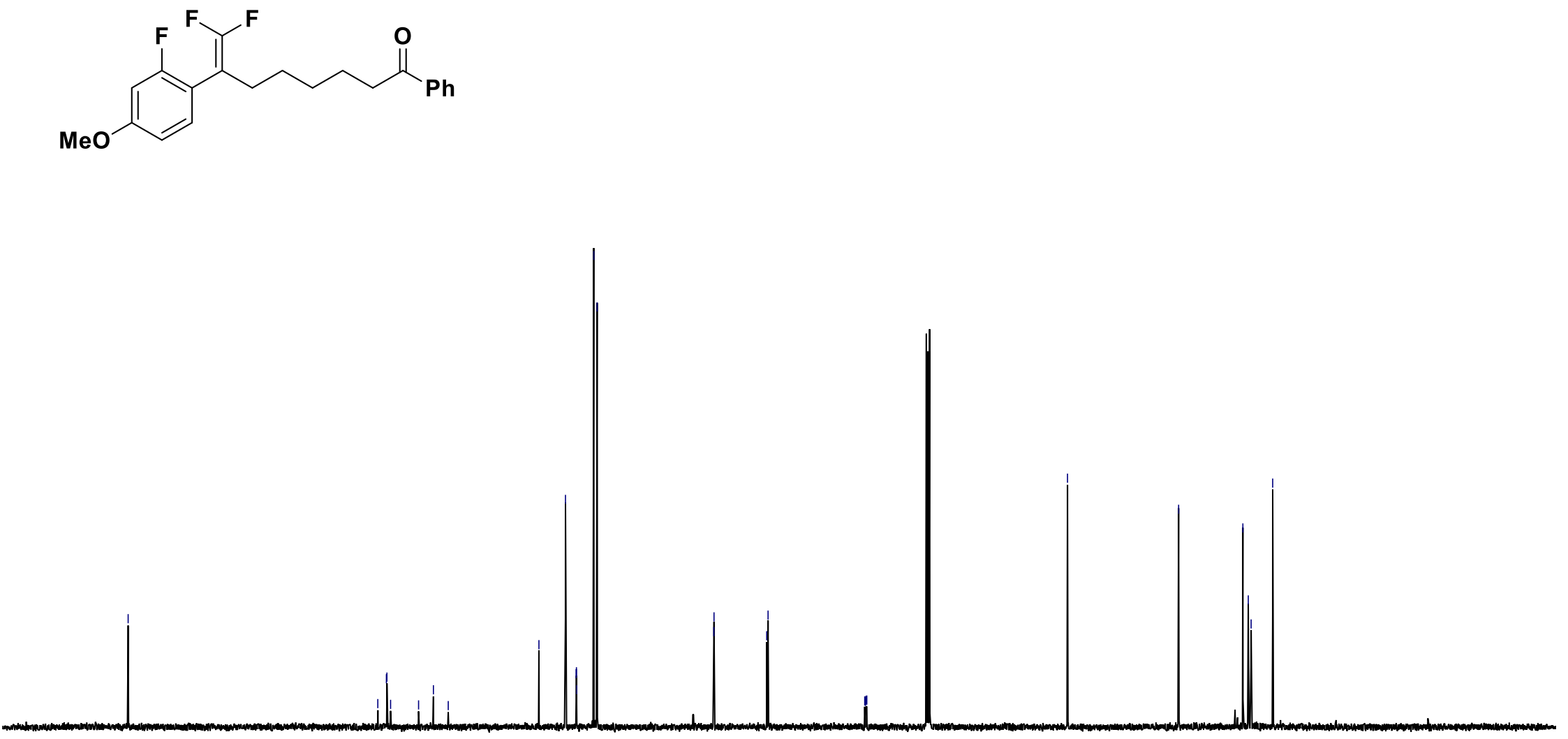

210200

$\begin{array}{llllllllllll}190 & 180 & 170 & 160 & 150 & 140 & 130 & 120 & 110 & 100 & 90\end{array}$ f1 (ppm) 
${ }^{19} \mathrm{~F}$ NMR-spectrum $\left(376 \mathrm{MHz}, \mathrm{CDCl}_{3}\right)$ of $3 \mathbf{l a}$

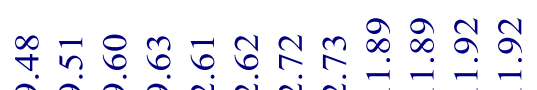

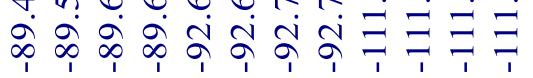
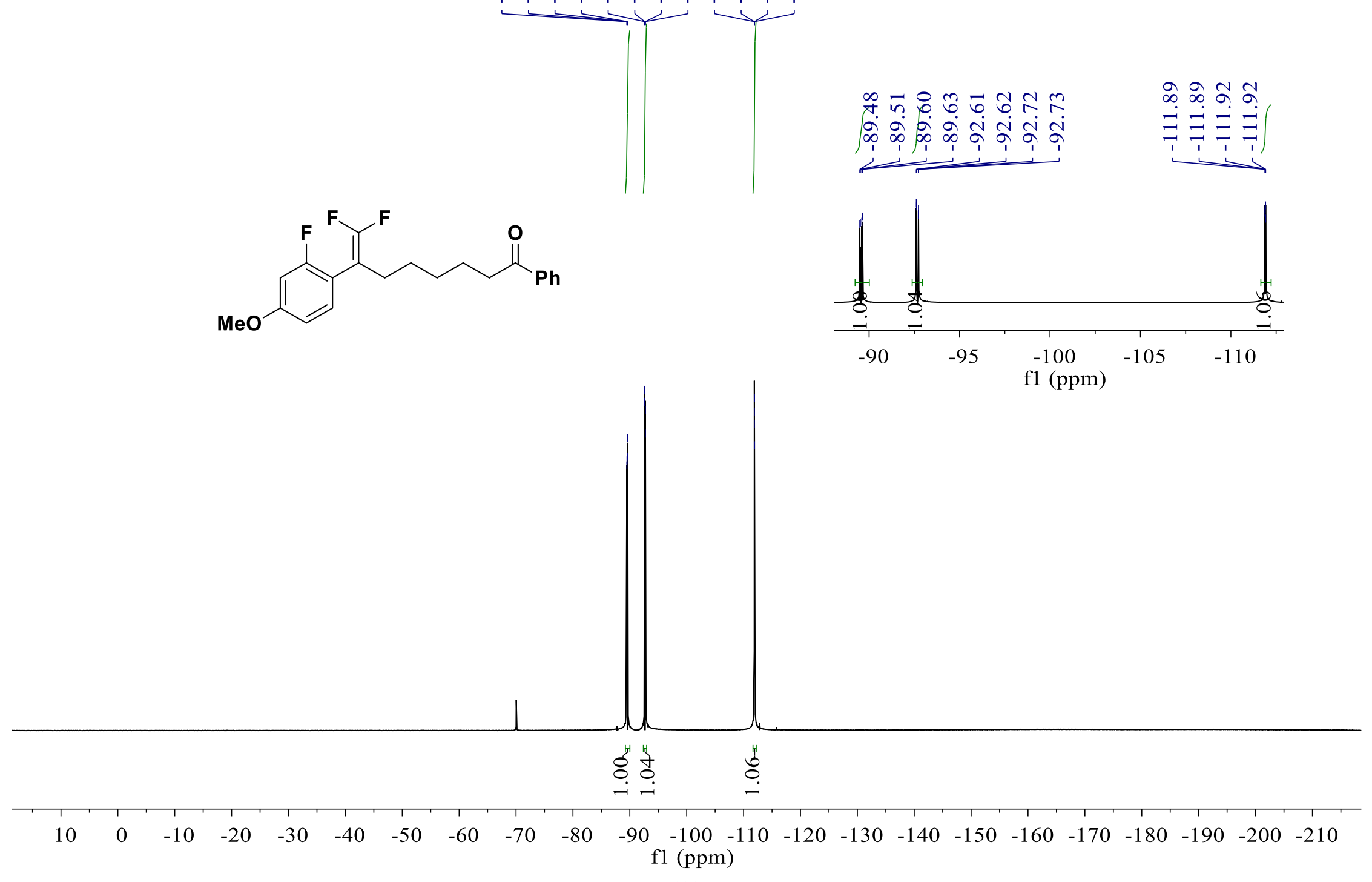

S38 


\section{${ }^{1} \mathrm{H}$ NMR-spectrum $\left(500 \mathrm{MHz}, \mathrm{CDCl}_{3}\right)$ of $\mathbf{3 m a}$}

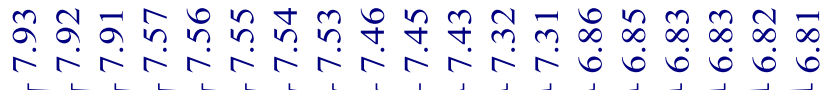
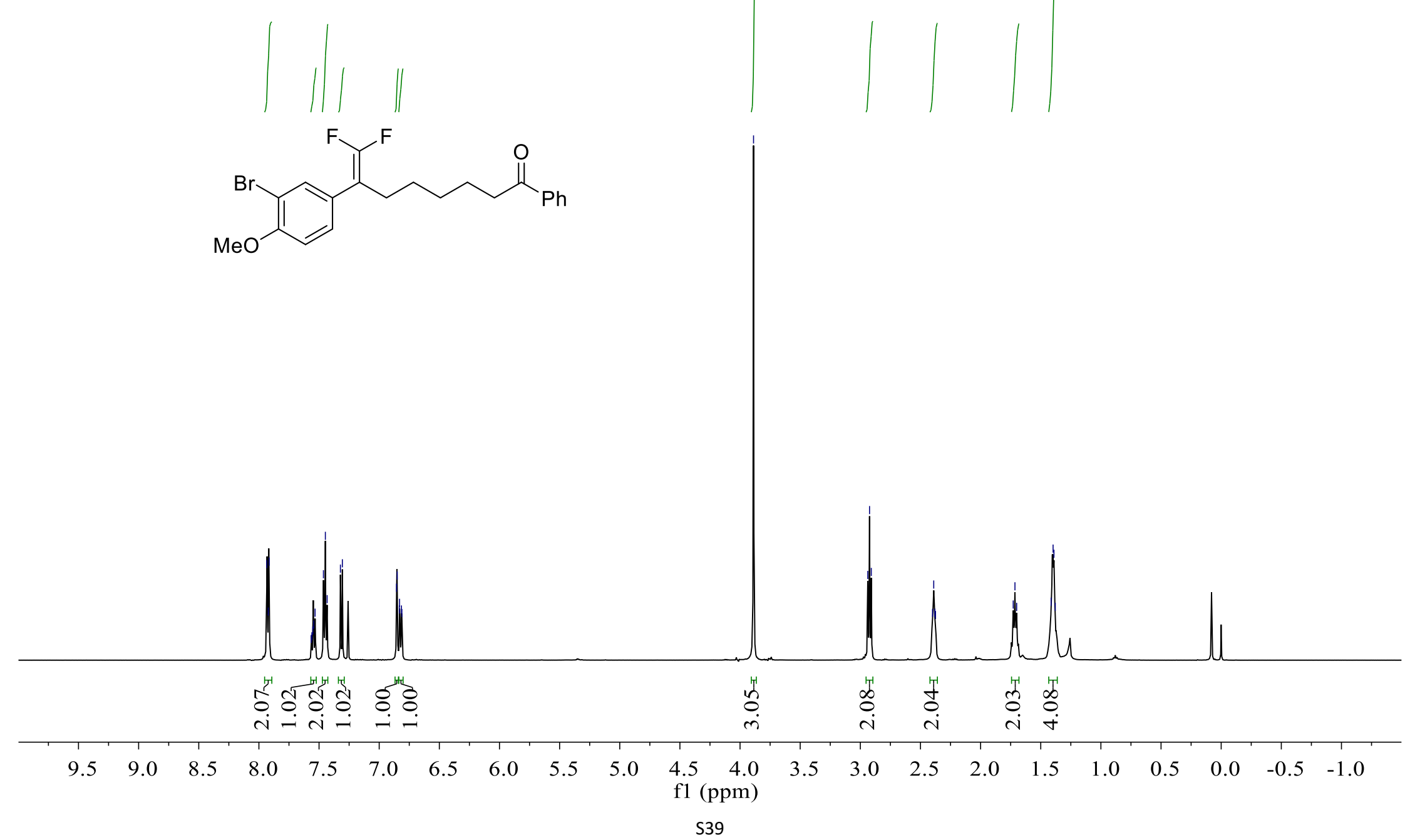

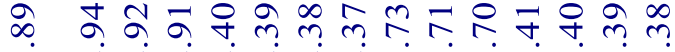

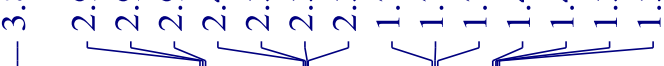


${ }^{13} \mathrm{C}\left\{{ }^{1} \mathrm{H}\right\}$ NMR-spectrum $\left(126 \mathrm{MHz}, \mathrm{CDCl}_{3}\right)$ of $\mathbf{3 m a}$

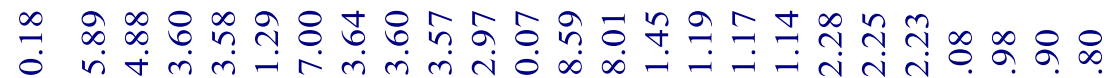

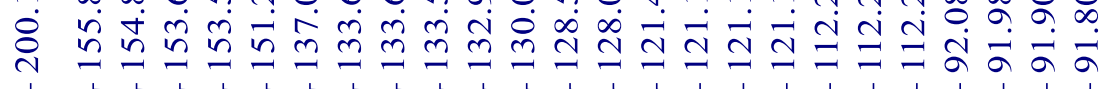

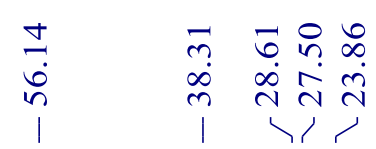
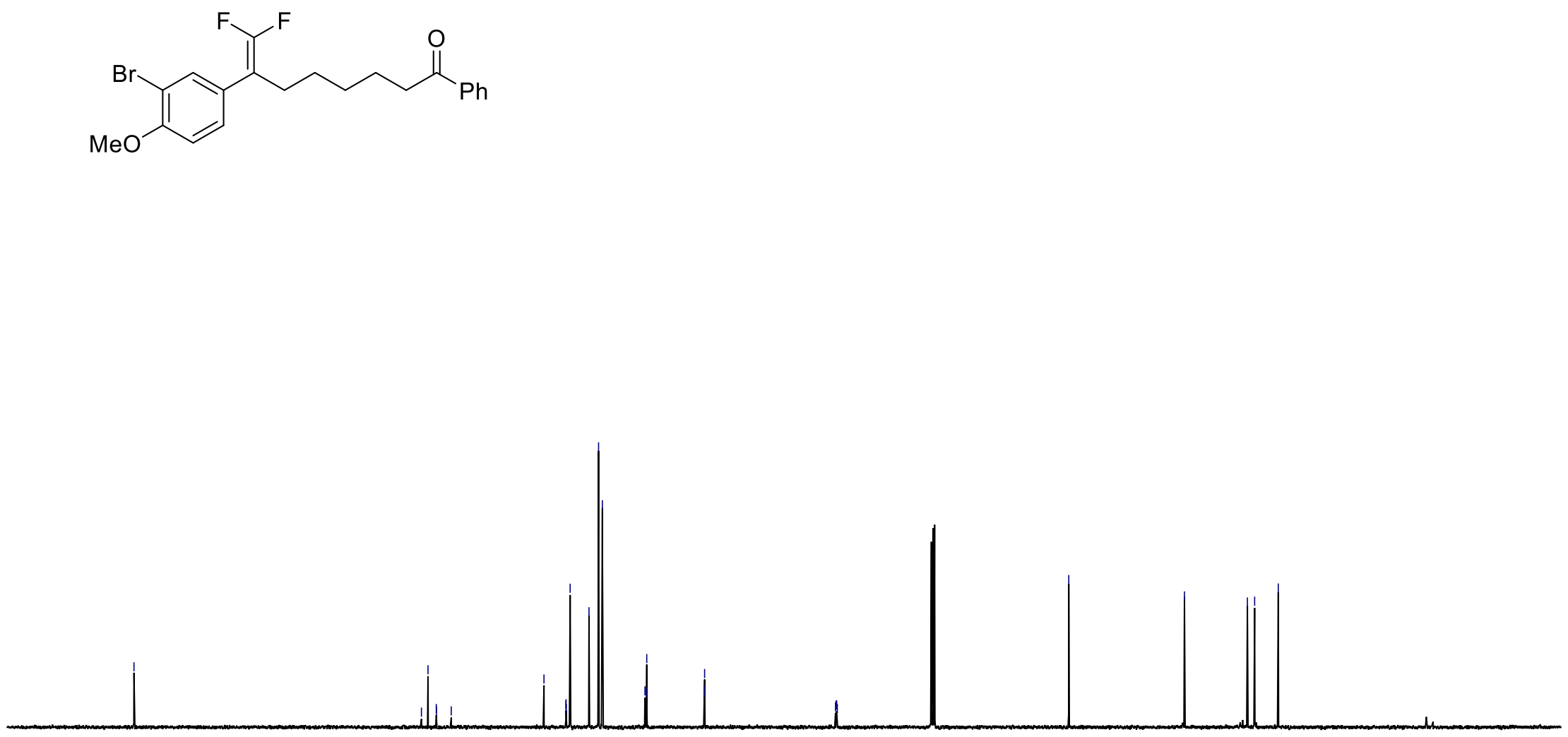

$\begin{array}{lllllllllllll}210 & 200 & 190 & 180 & 170 & 160 & 150 & 140 & 130 & 120 & 110 & 100 & 90\end{array}$

f1 (ppm)

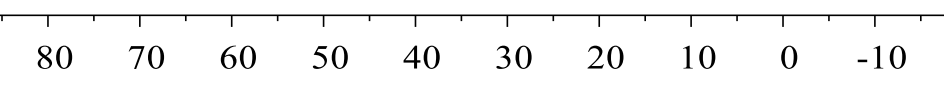

S40 
${ }^{19} \mathrm{~F}$ NMR-spectrum $\left(471 \mathrm{MHz}, \mathrm{CDCl}_{3}\right)$ of $\mathbf{3 m a}$

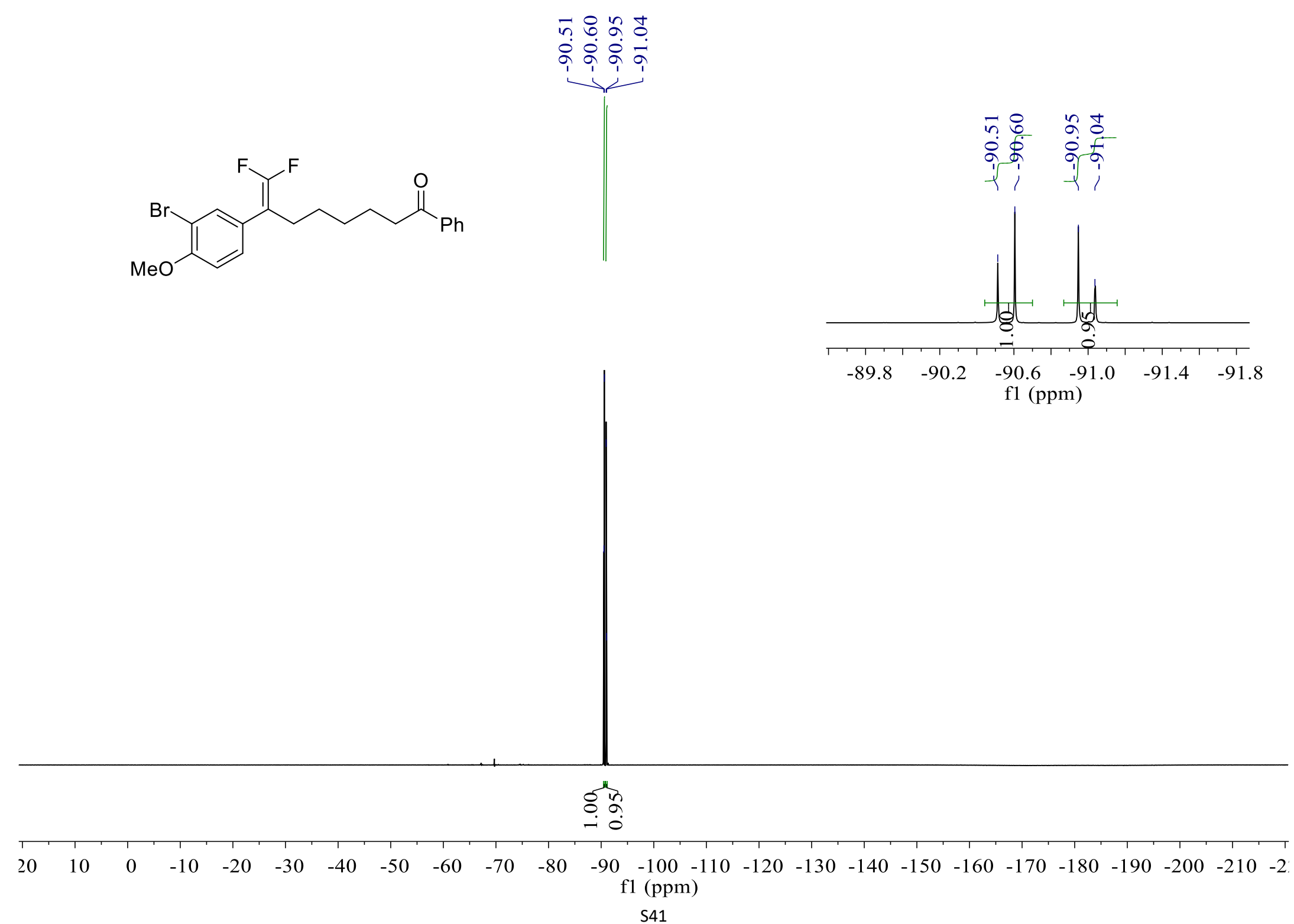



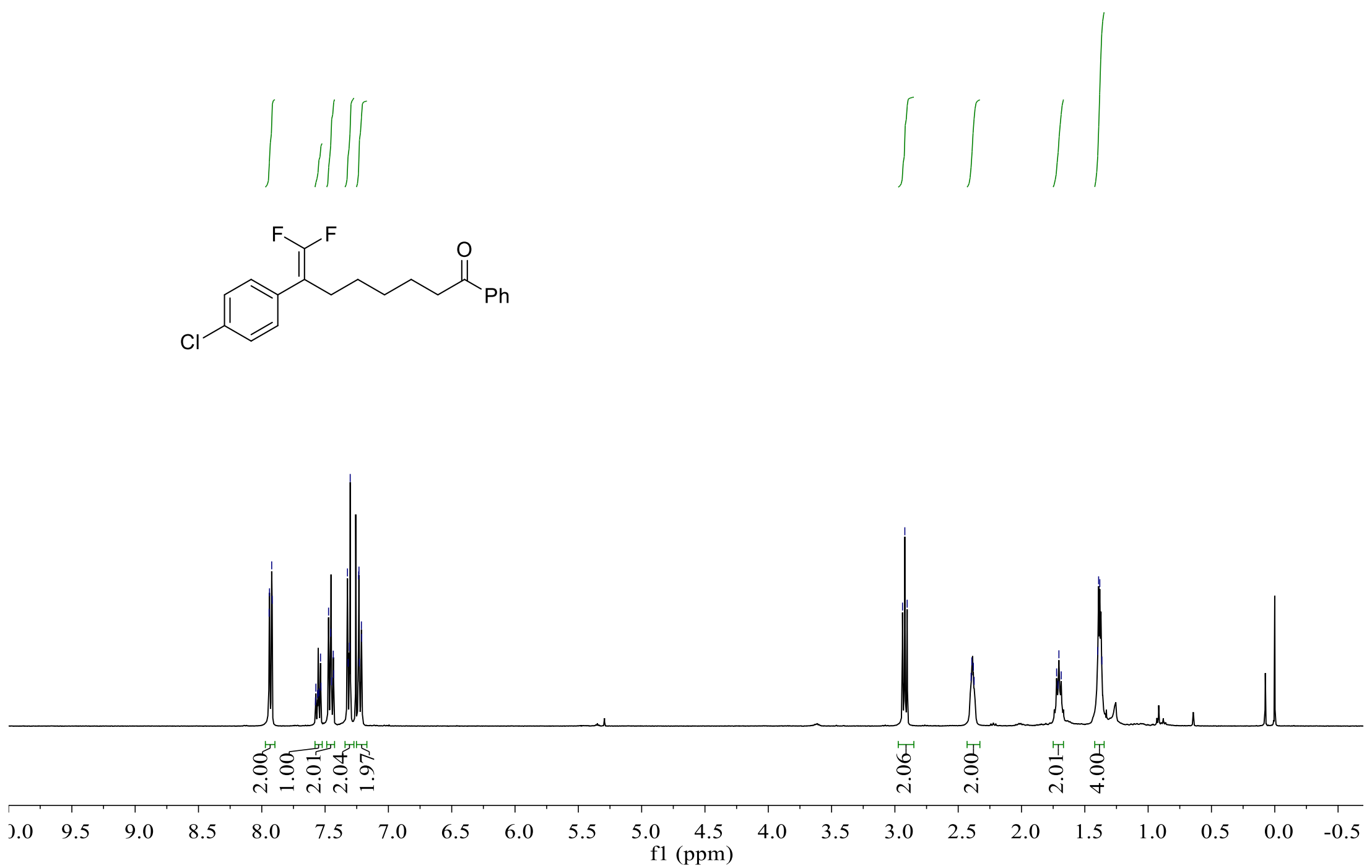
${ }^{13} \mathrm{C}\left\{{ }^{1} \mathrm{H}\right\}$ NMR-spectrum (400 MHz, $\left.\mathrm{CDCl}_{3}\right)$ of 3na

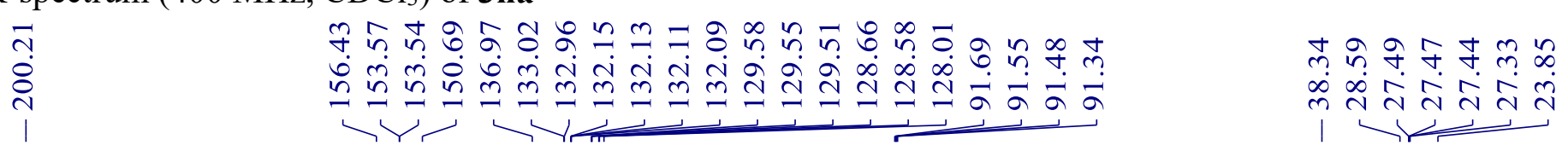
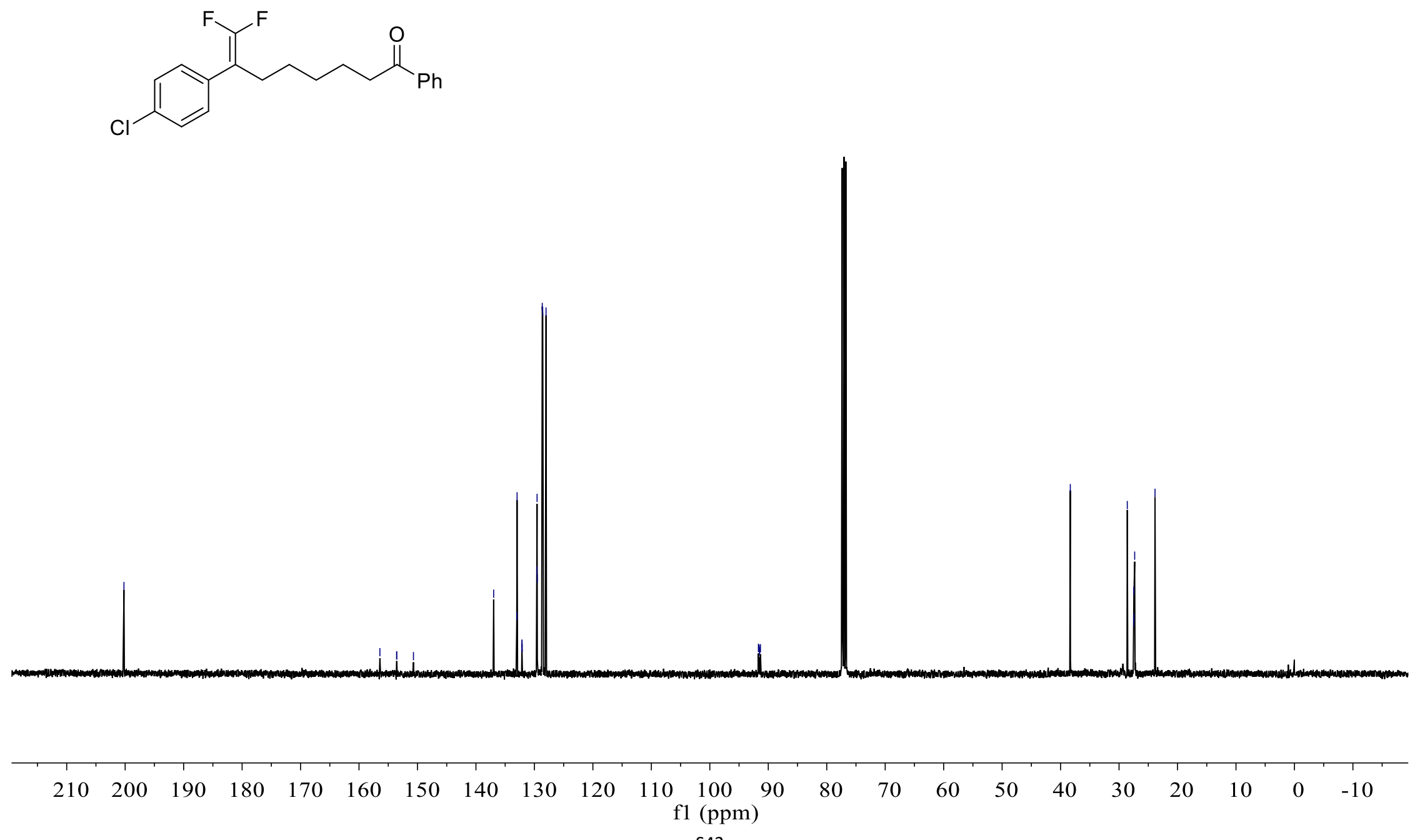
${ }^{19} \mathrm{~F}$ NMR-spectrum $\left(376 \mathrm{MHz}, \mathrm{CDCl}_{3}\right.$ ) of 3na

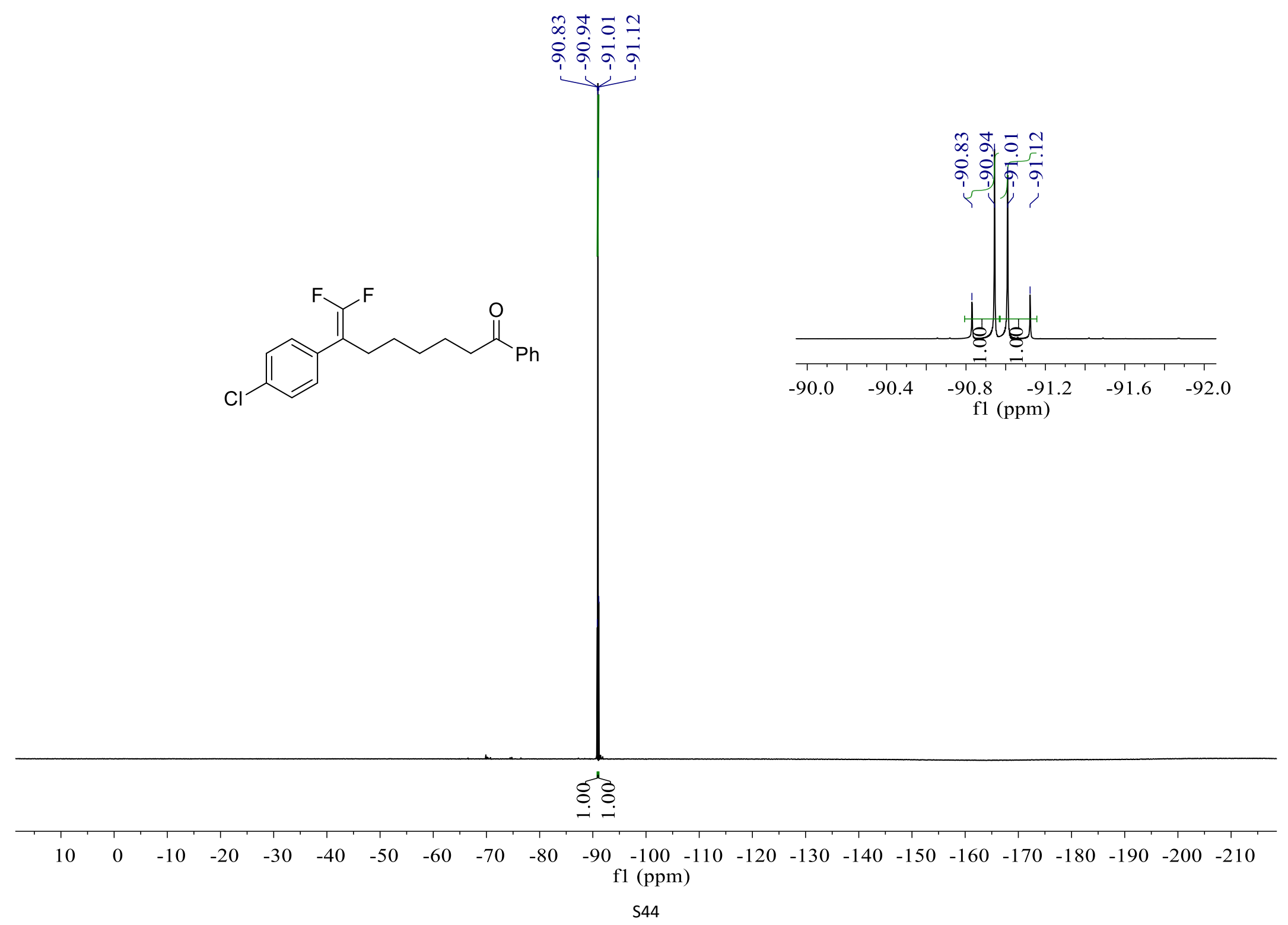




\section{${ }^{1} \mathrm{H}$ NMR-spectrum $\left(500 \mathrm{MHz}, \mathrm{CDCl}_{3}\right)$ of $\mathbf{3 0 a}$}
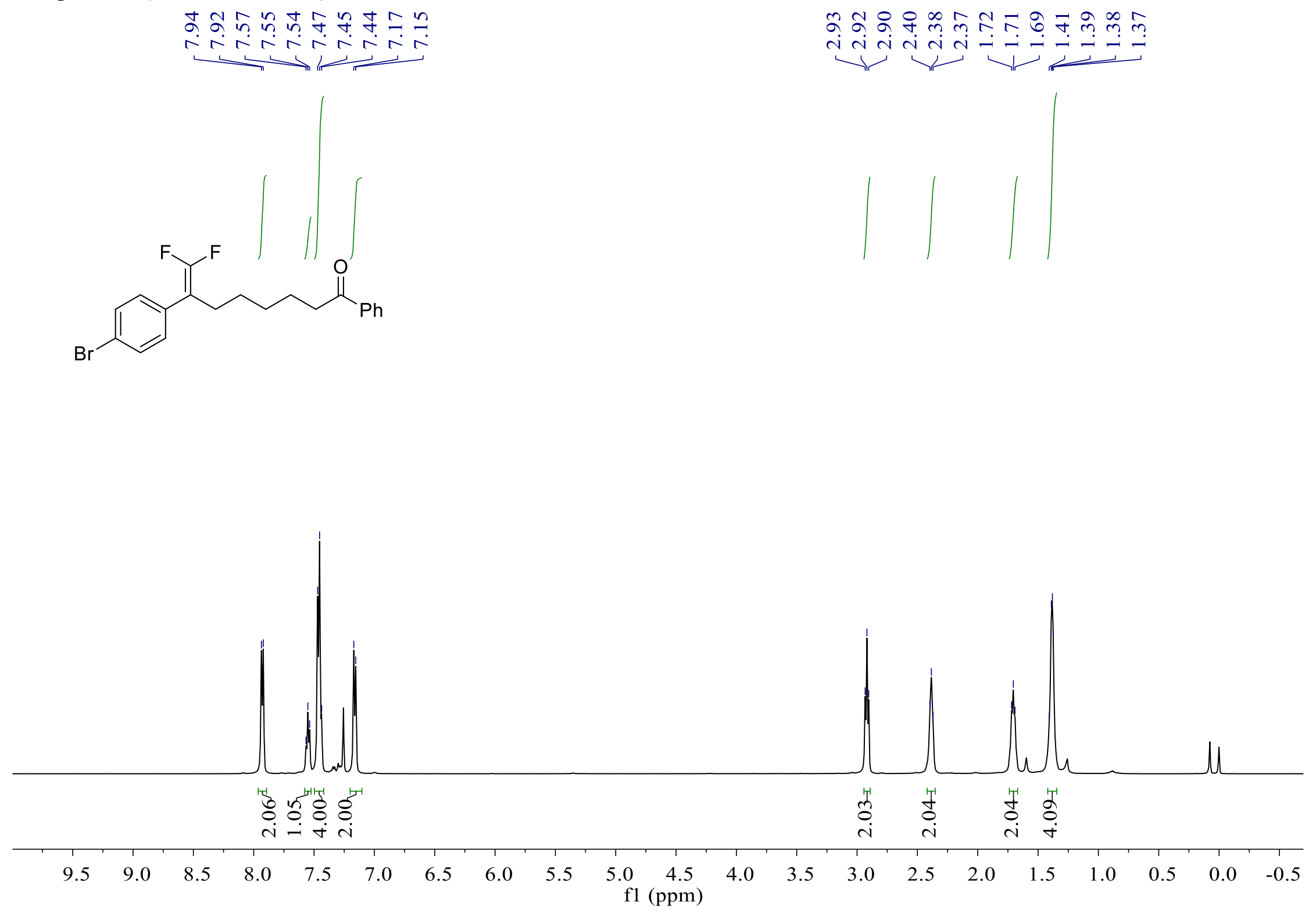

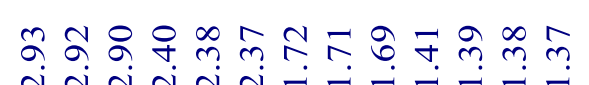

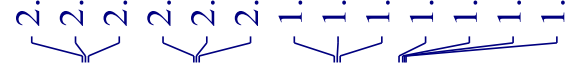

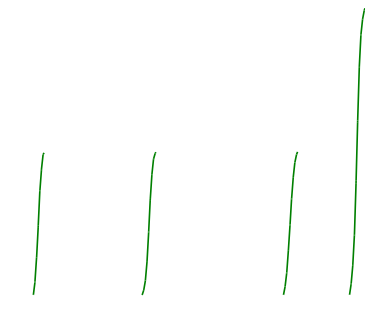

S45 
${ }^{13} \mathrm{C}\left\{{ }^{1} \mathrm{H}\right\}$ NMR-spectrum (126 MHz, $\left.\mathrm{CDCl}_{3}\right)$ of $30 a$

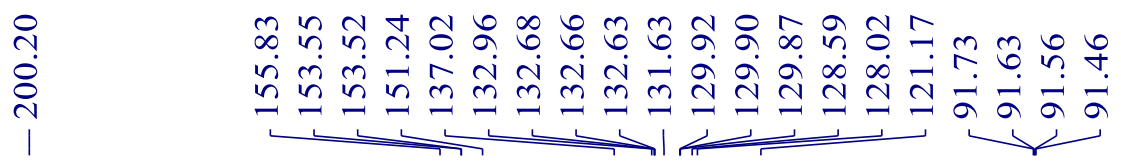

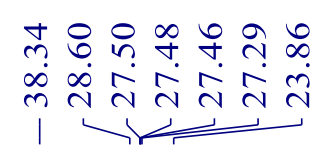<smiles>O=C(CCCCCC(=C(F)F)c1ccc(Br)cc1)c1ccccc1</smiles>
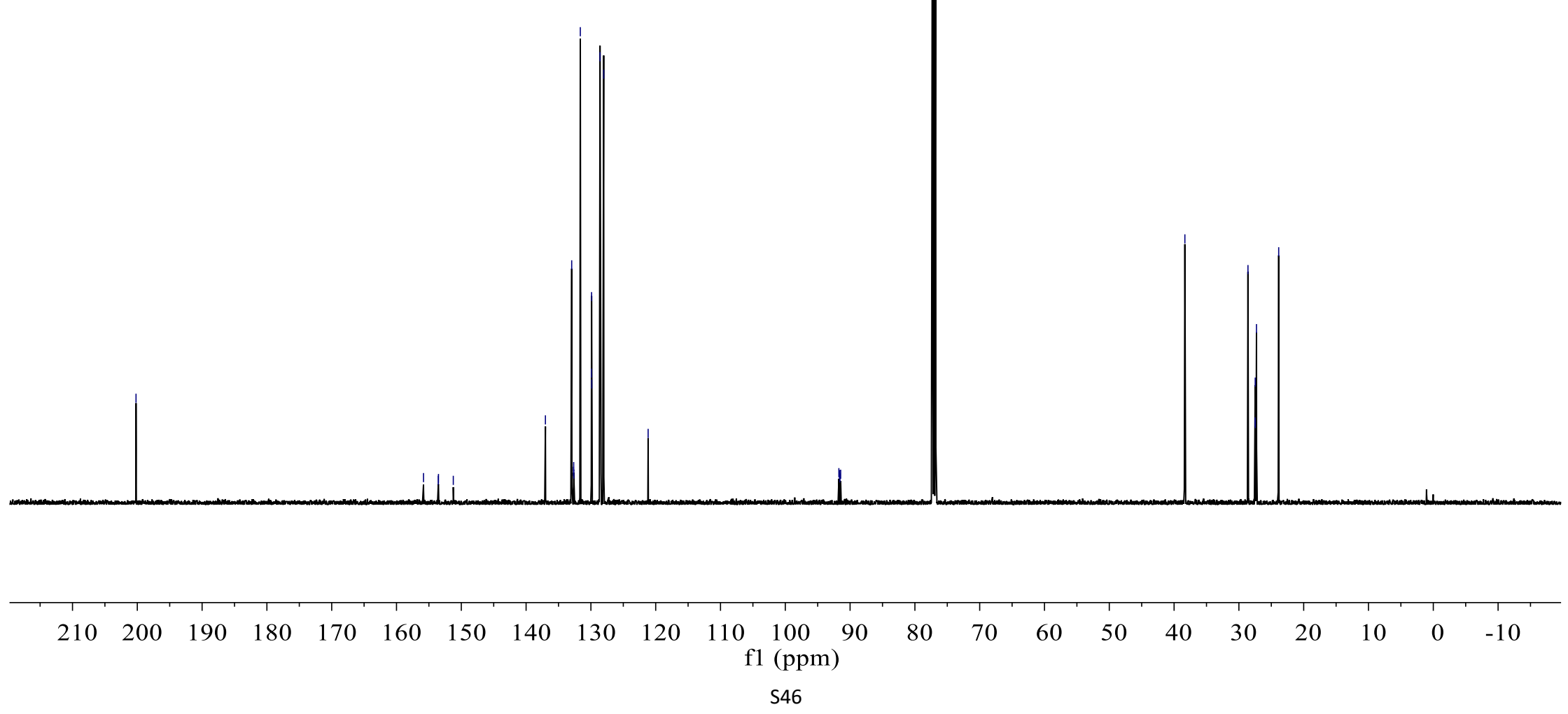
${ }^{19} \mathrm{~F}$ NMR-spectrum $\left(471 \mathrm{MHz}, \mathrm{CDCl}_{3}\right)$ of $\mathbf{3 o a}$

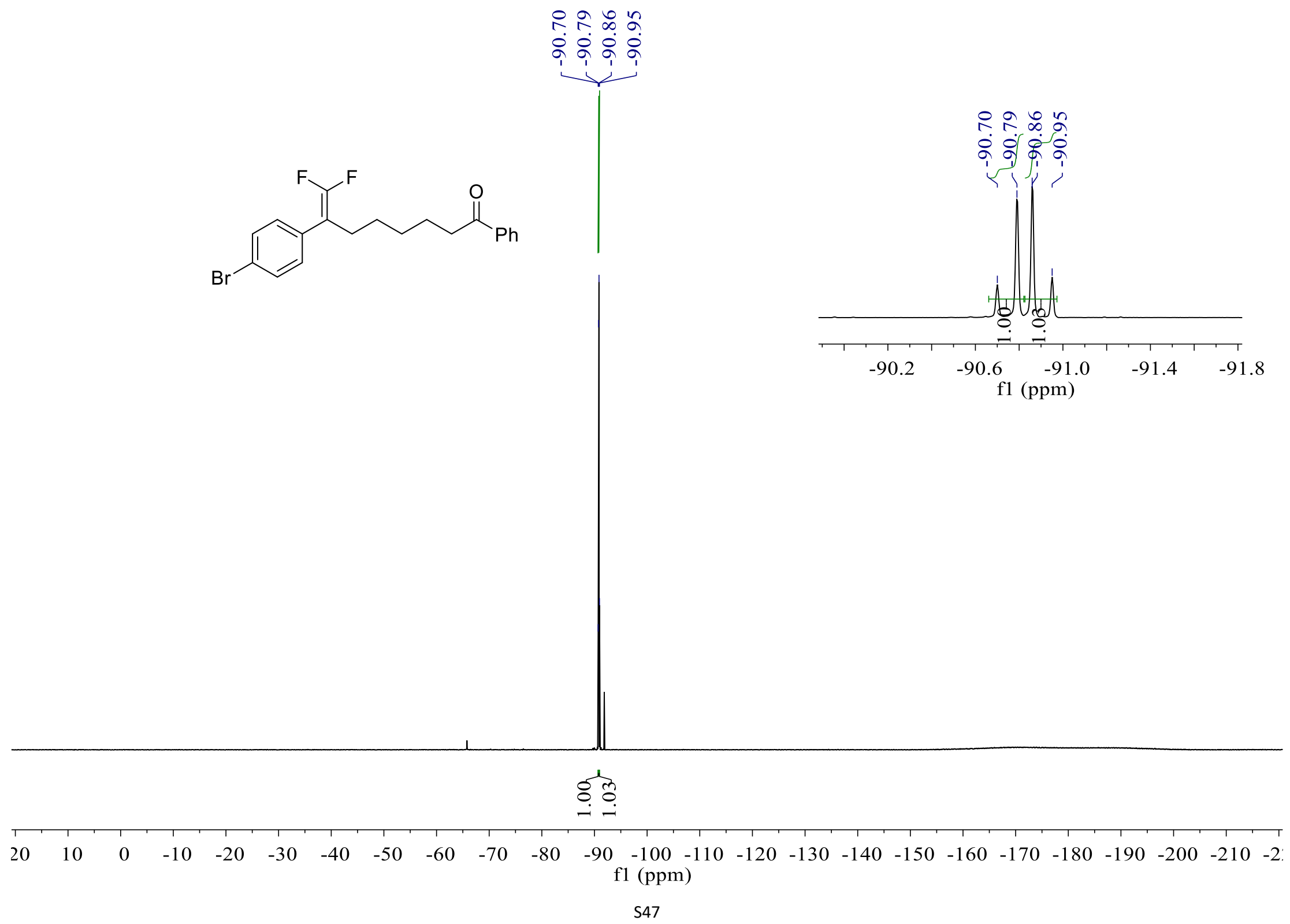




\section{${ }^{1} \mathrm{H}$ NMR-spectrum $\left(400 \mathrm{MHz}, \mathrm{CDCl}_{3}\right)$ of 3pa}

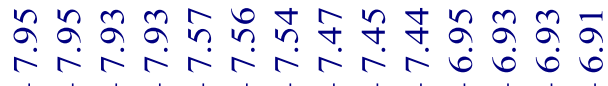

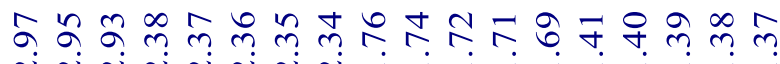
ن त
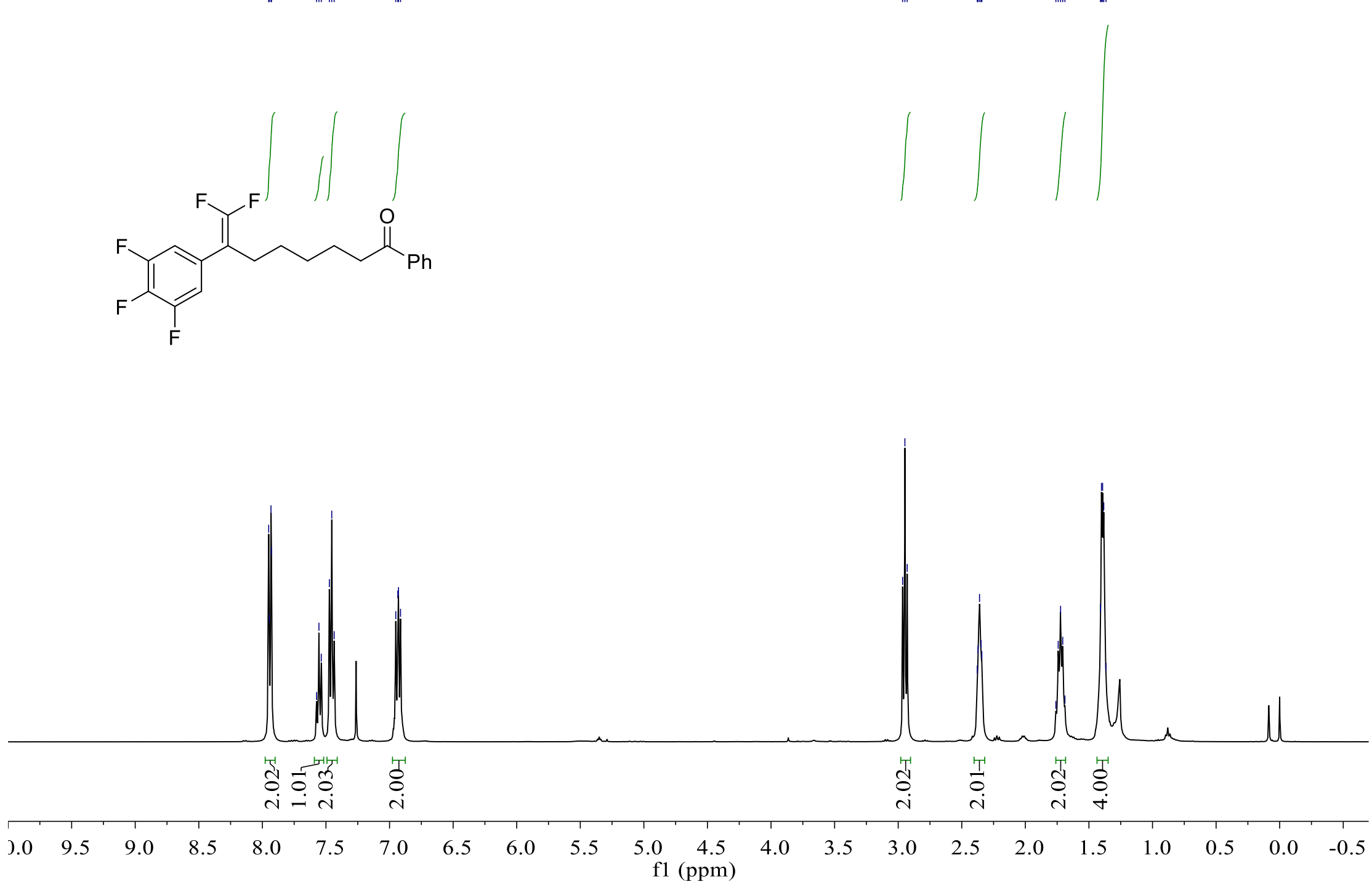

\$48 
${ }^{13} \mathrm{C}\left\{{ }^{1} \mathrm{H}\right\}$ NMR-spectrum $\left(126 \mathrm{MHz}, \mathrm{CDCl}_{3}\right)$ of $\mathbf{3 p a}$

동

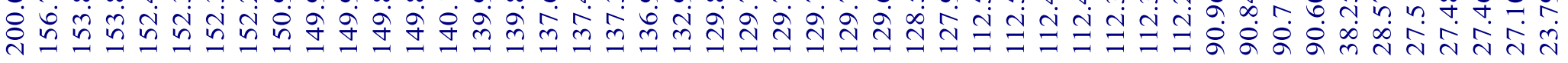
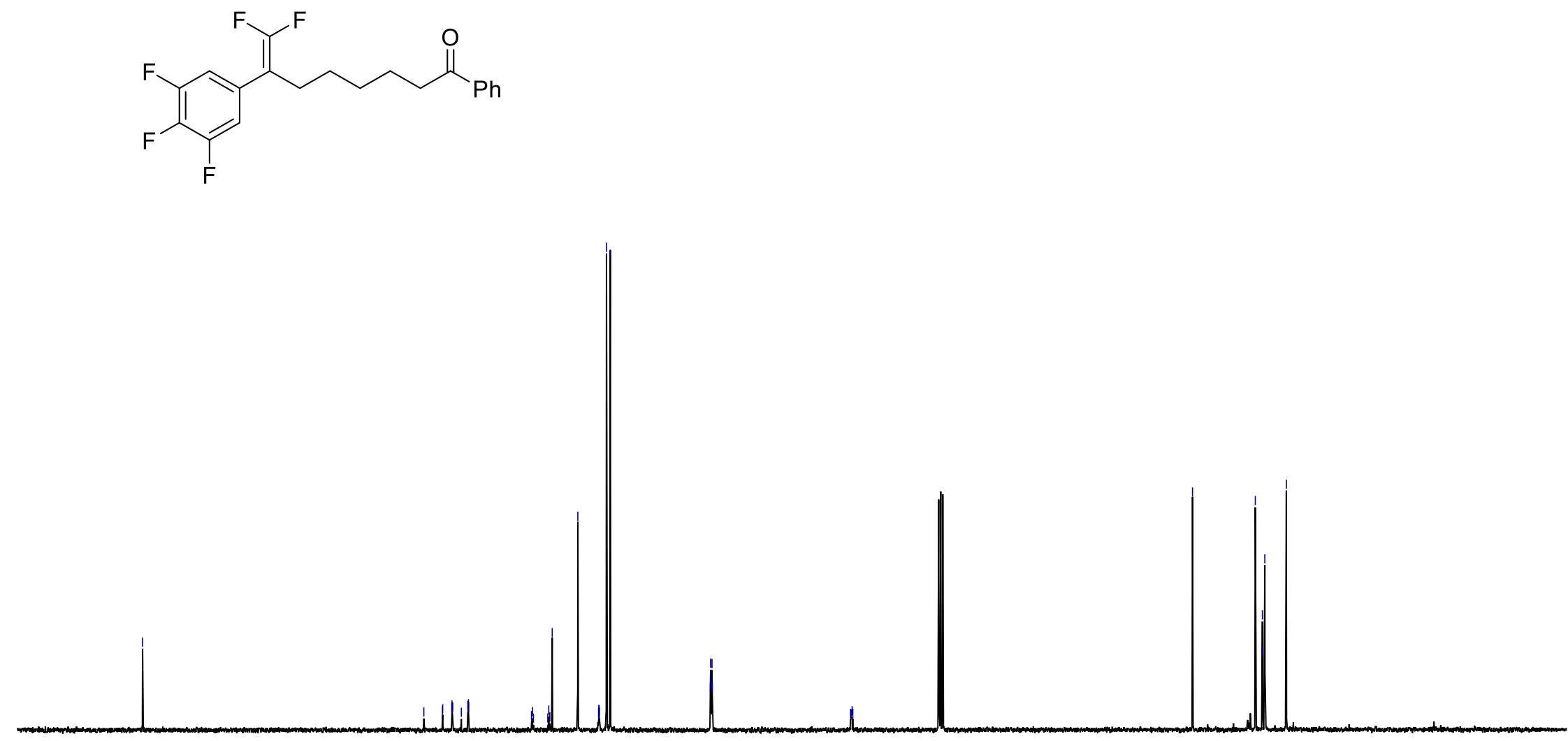

$\begin{array}{llllllllllll}210 & 200 & 190 & 180 & 170 & 160 & 150 & 140 & 130 & 120 & 110 & 100\end{array}$

f1 (ppm)

$-10$

S49 
${ }^{19} \mathrm{~F}$ NMR-spectrum $\left(376 \mathrm{MHz}, \mathrm{CDCl}_{3}\right)$ of 3 pa
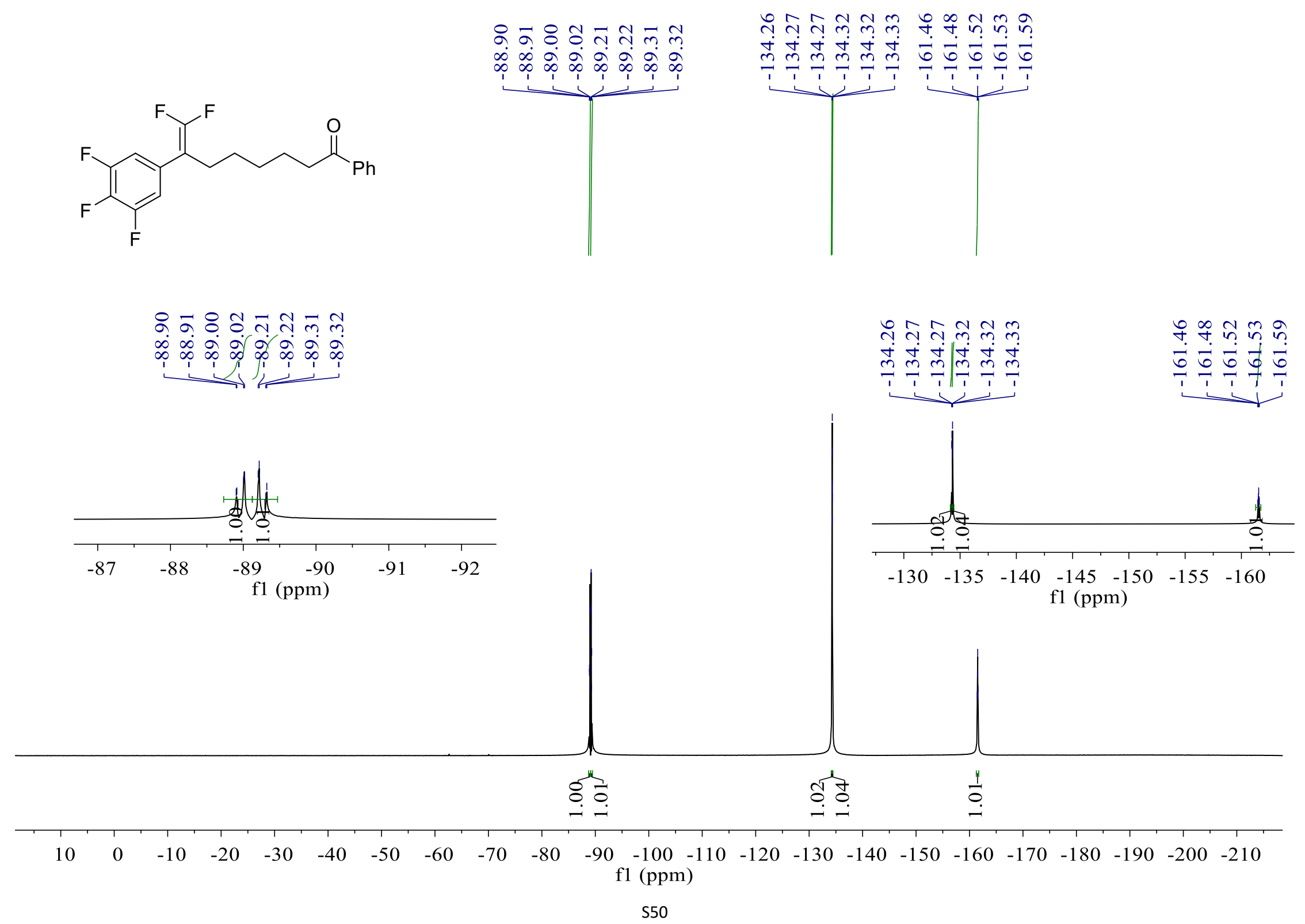


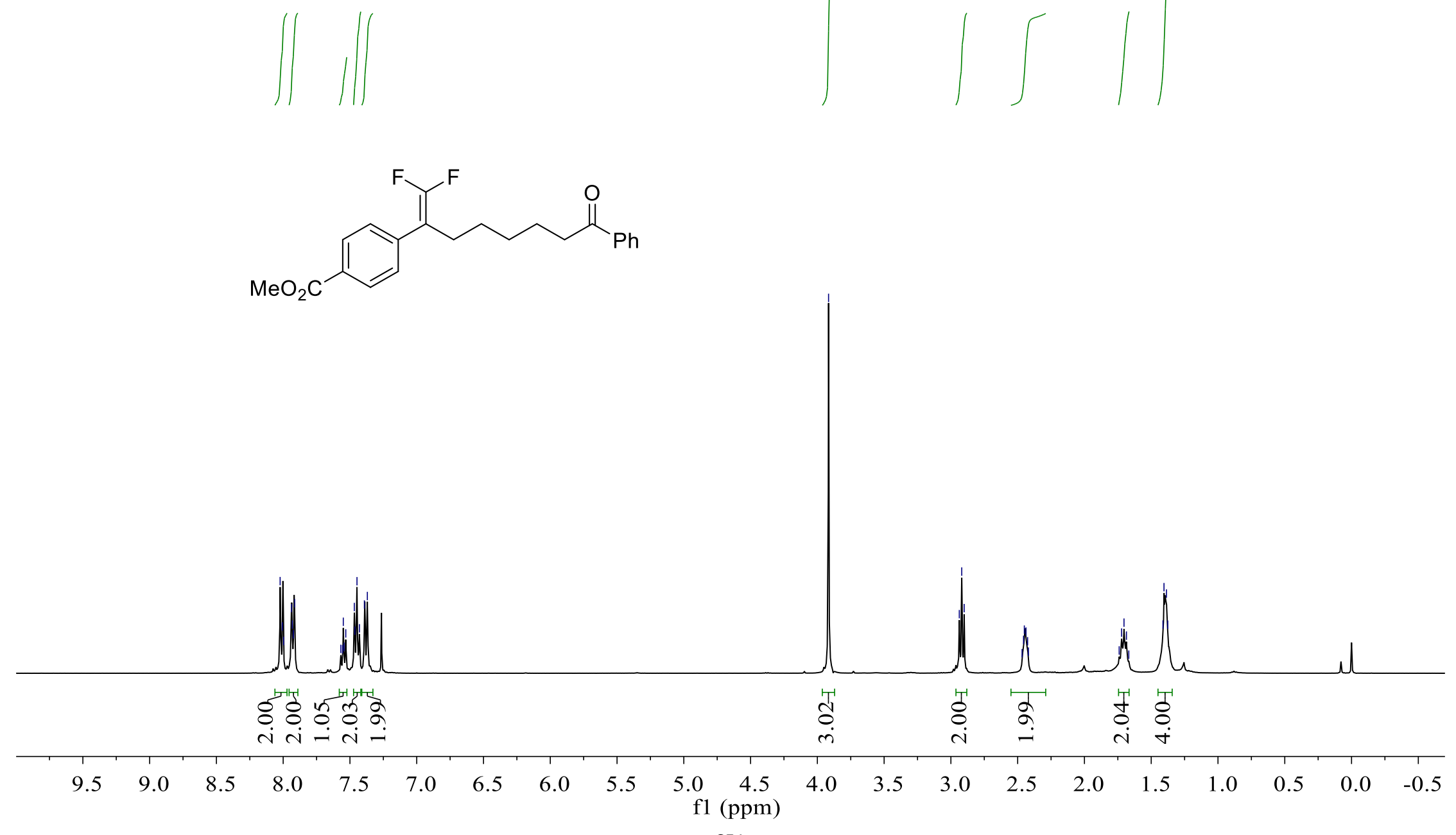


${ }^{13} \mathrm{C}\left\{{ }^{1} \mathrm{H}\right\}$ NMR-spectrum $\left(101 \mathrm{MHz}, \mathrm{CDCl}_{3}\right)$ of $\mathbf{3 q a}$

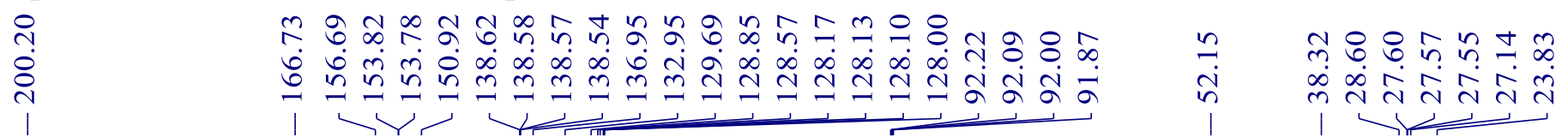

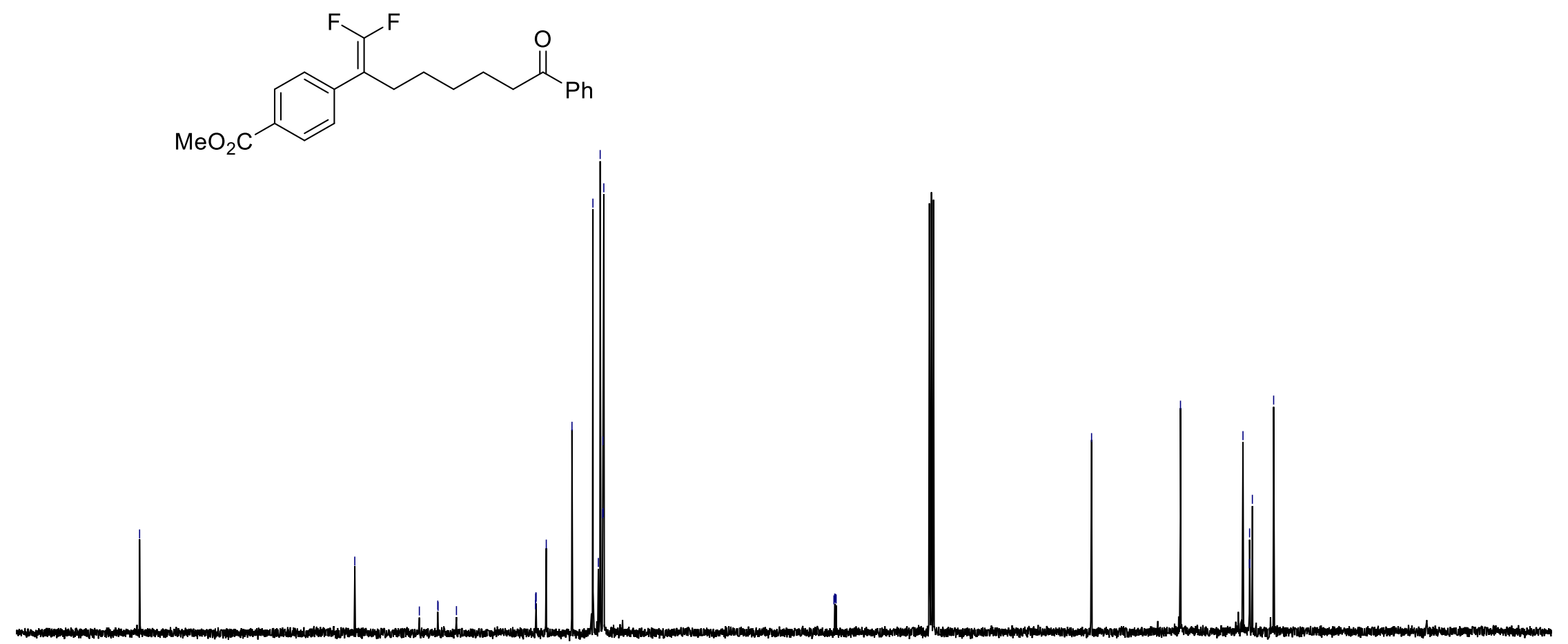

$\begin{array}{llllllllllllllllllllll}210 & 200 & 190 & 180 & 170 & 160 & 150 & 140 & 130 & 120 & \begin{array}{c}110 \\ \mathrm{fl} 1(\mathrm{ppm})\end{array} & 100 & 90 & 70 & 60 & 50 & 40 & 30 & 20 & 10 & 0 & -10\end{array}$

S52 
${ }^{19} \mathrm{~F}$ NMR-spectrum $\left(376 \mathrm{MHz}, \mathrm{CDCl}_{3}\right.$ ) of $\mathbf{3 q a}$

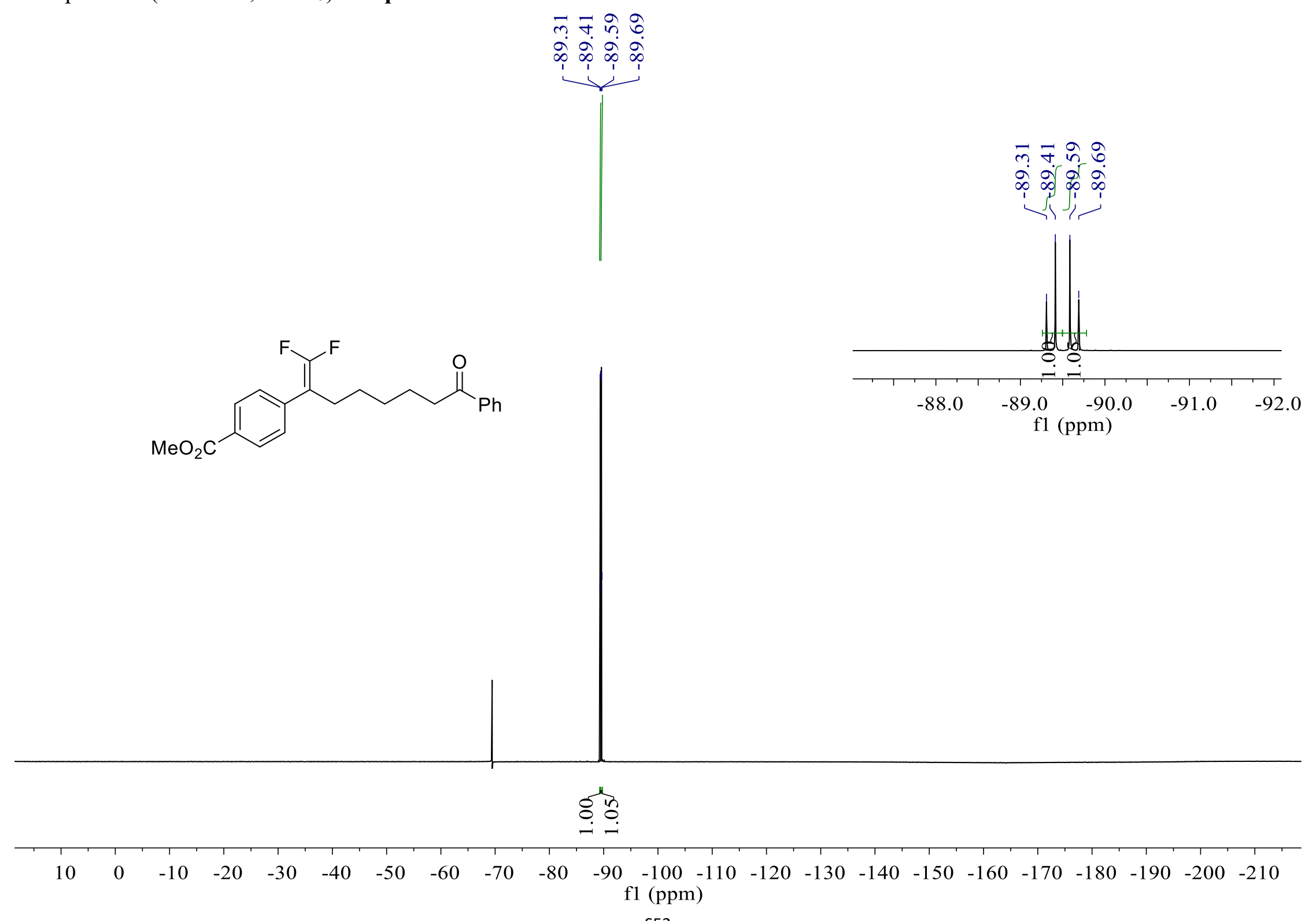




\section{${ }^{1} \mathrm{H}$ NMR-spectrum $\left(400 \mathrm{MHz}, \mathrm{CDCl}_{3}\right)$ of 3ra}

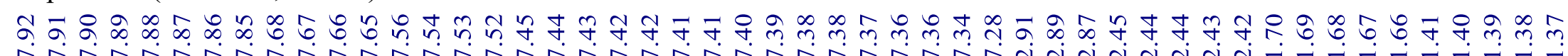
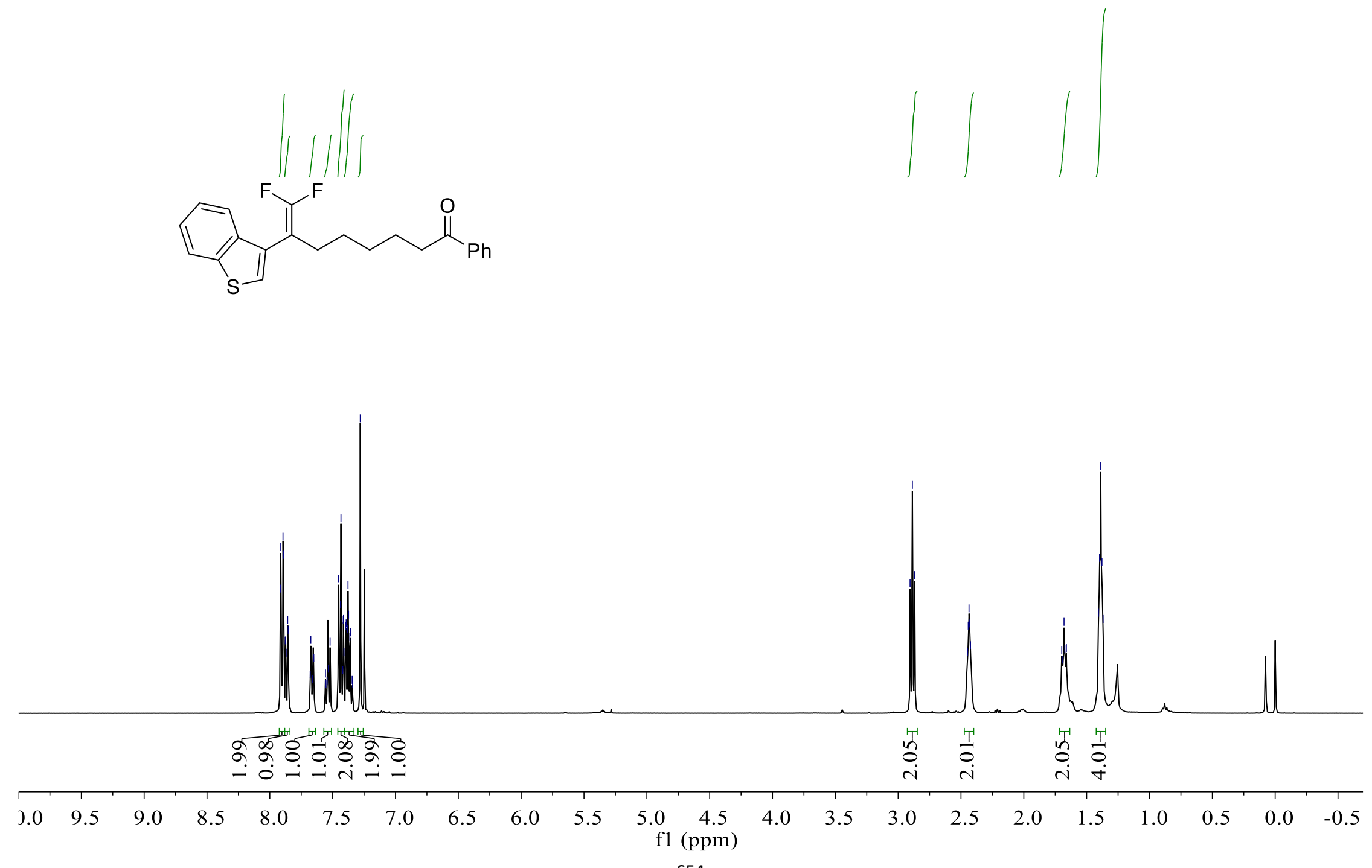

S54 
${ }^{13} \mathrm{C}\left\{{ }^{1} \mathrm{H}\right\}$ NMR-spectrum $\left(126 \mathrm{MHz}, \mathrm{CDCl}_{3}\right)$ of 3ra

$\stackrel{n}{i}$

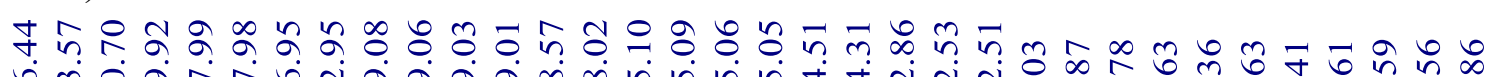

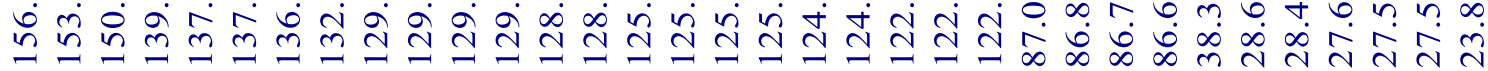

ᄀ;
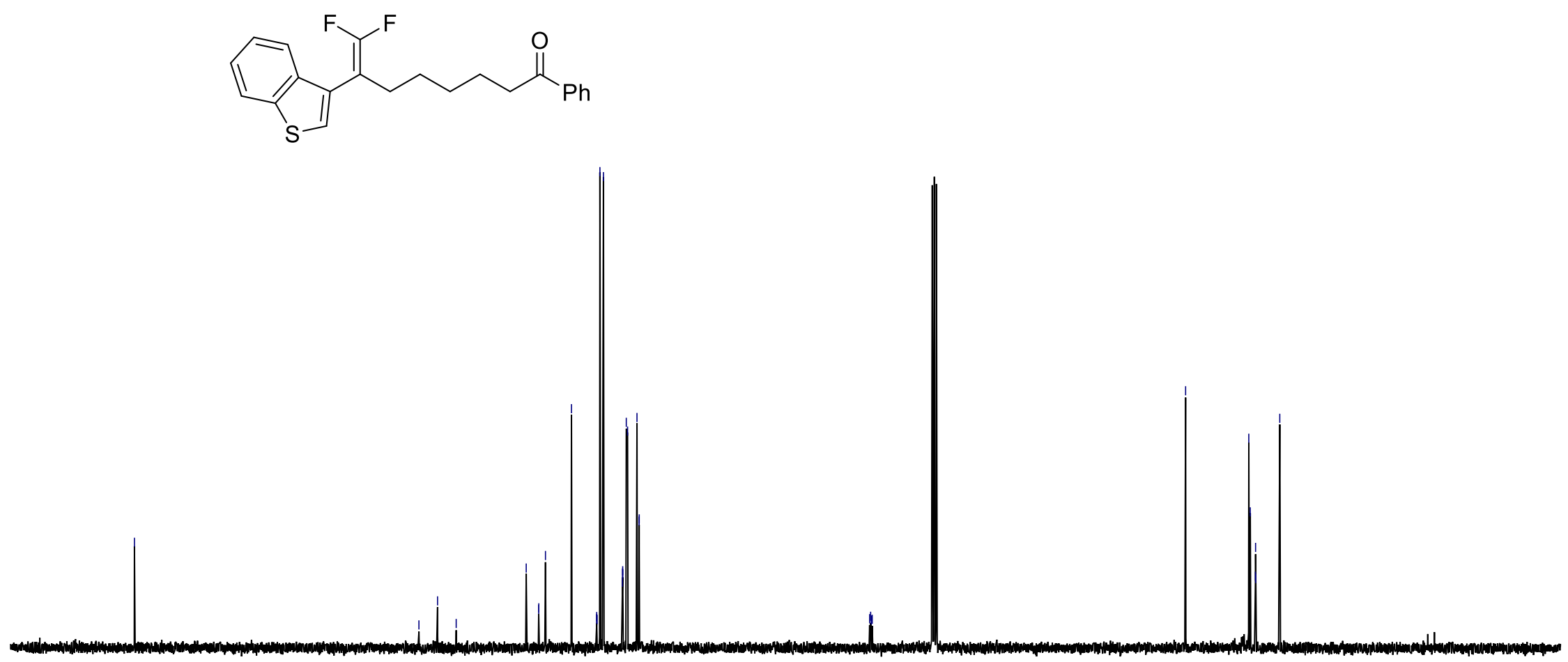

$\begin{array}{lllllllllllllllllllllll}210 & 200 & 190 & 180 & 170 & 160 & 150 & 140 & 130 & 120 & 110 & \begin{array}{c}100 \\ \mathrm{f} 1(\mathrm{ppm})\end{array} & 90 & 80 & 70 & 60 & 50 & 40 & 30 & 20 & 10 & 0 & -10\end{array}$ S55 
${ }^{19} \mathrm{~F}$ NMR-spectrum $\left(376 \mathrm{MHz}, \mathrm{CDCl}_{3}\right)$ of 3ra

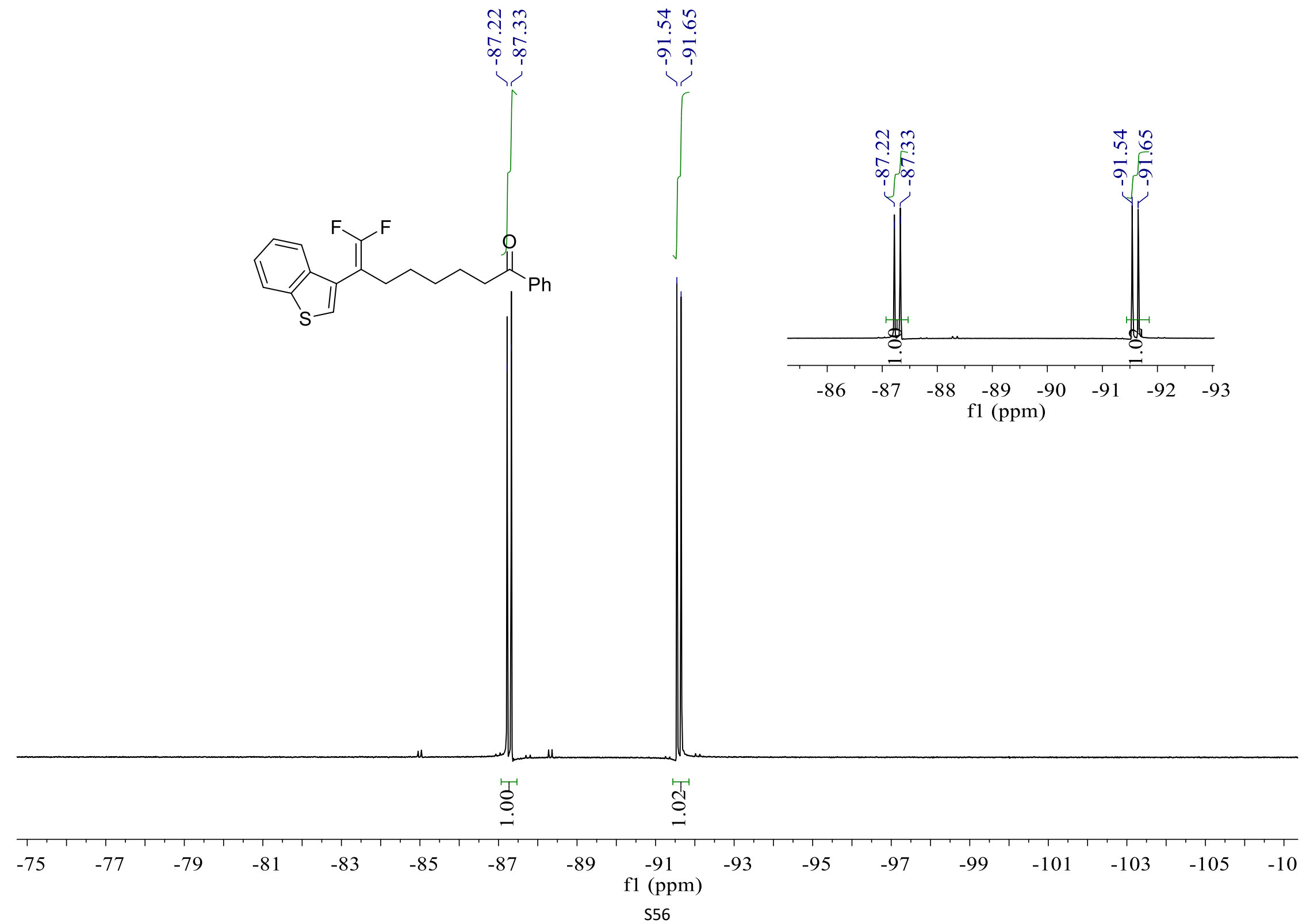




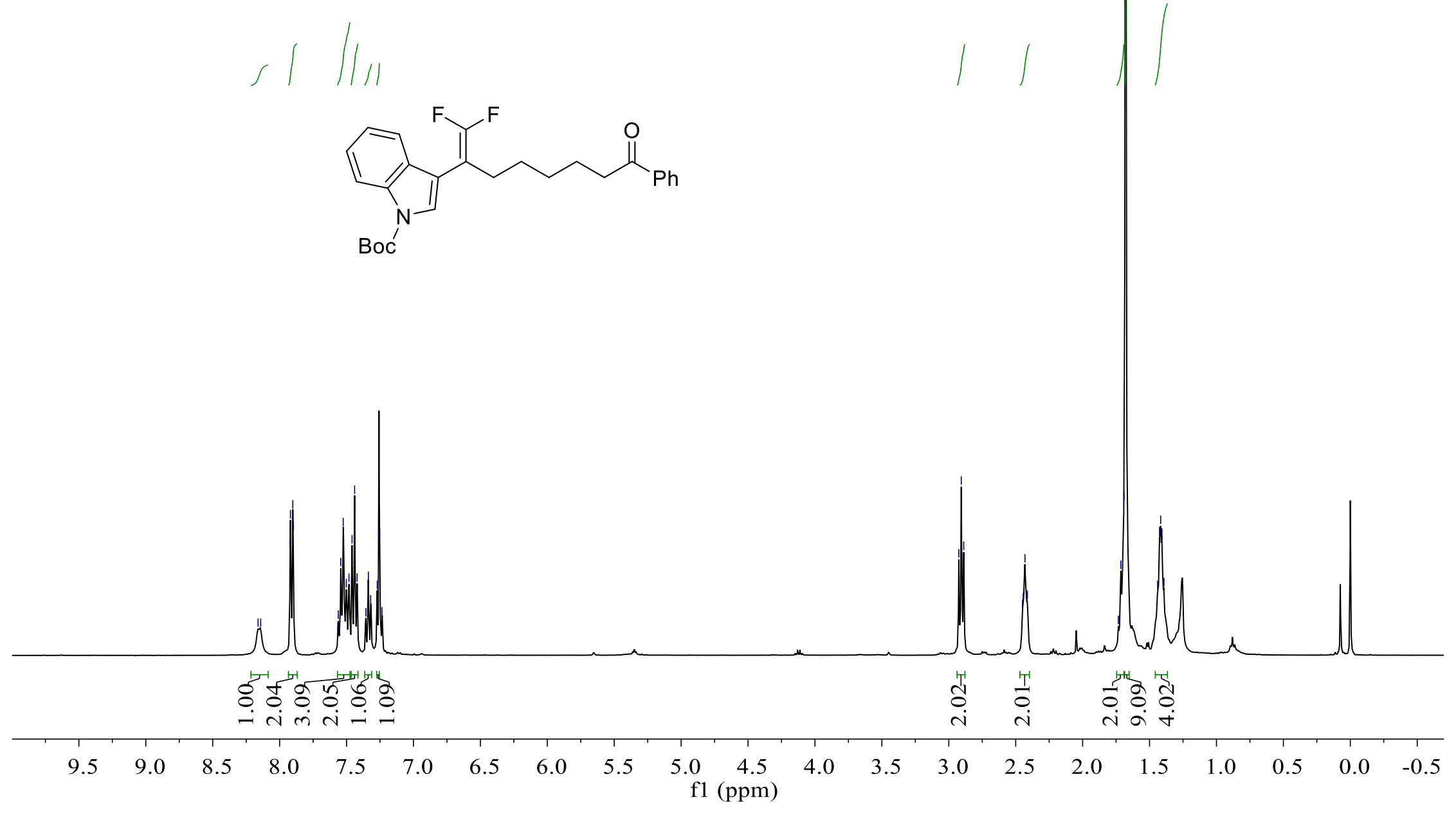

S57 
${ }^{13} \mathrm{C}\left\{{ }^{1} \mathrm{H}\right\}$ NMR-spectrum $\left(126 \mathrm{MHz}, \mathrm{CDCl}_{3}\right)$ of 3sa

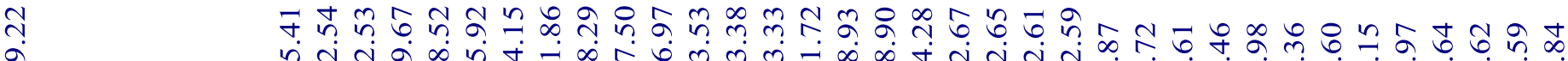

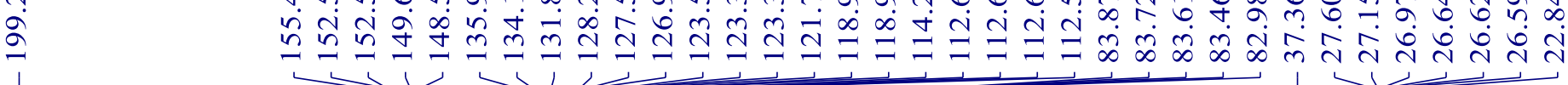
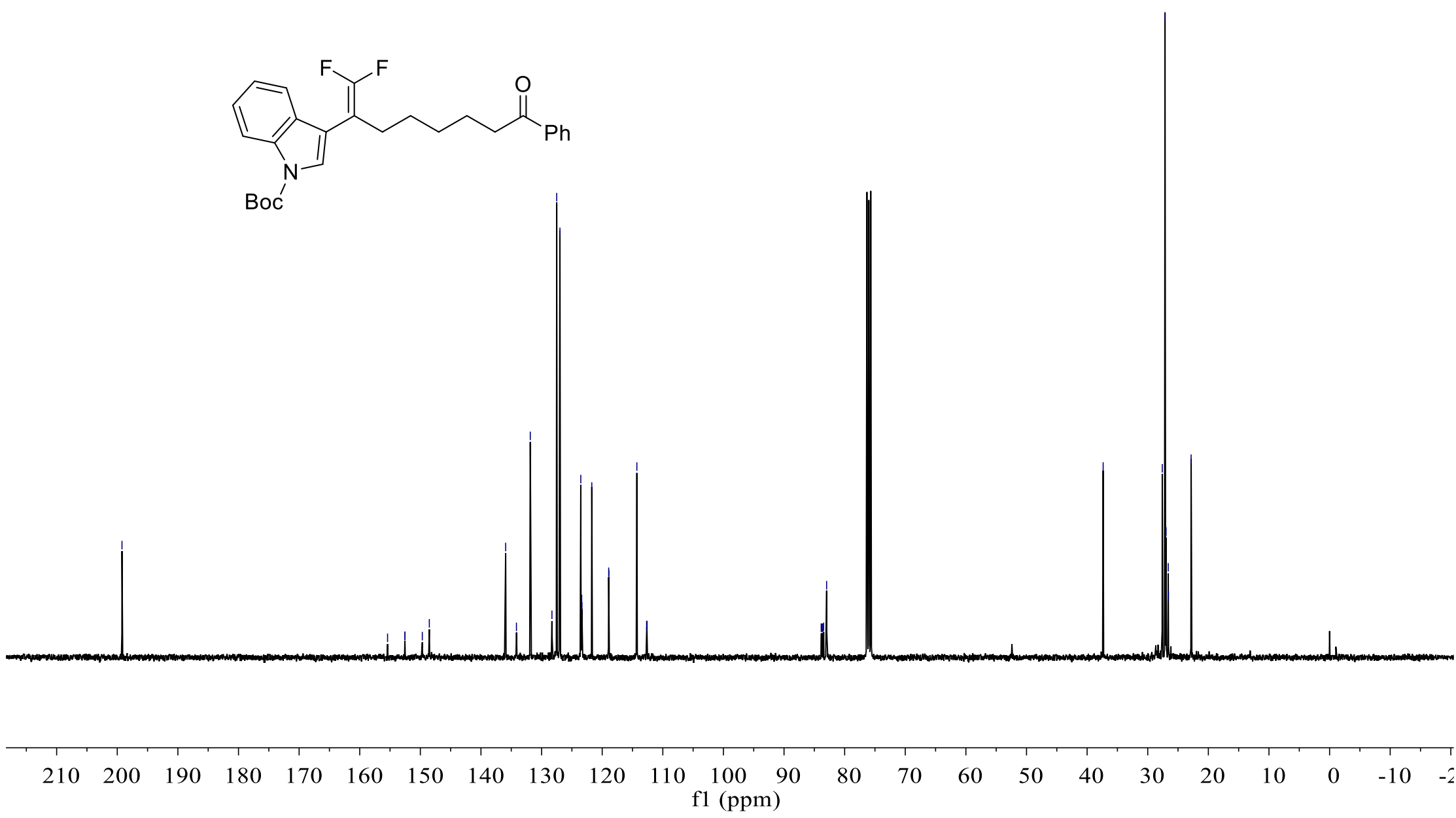

$\begin{array}{lllllllllllll}210 & 200 & 190 & 180 & 170 & 160 & 150 & 140 & 130 & 120 & 110 & 100 & 90\end{array}$

f1 (ppm) 
${ }^{19} \mathrm{H}$ NMR-spectrum $\left(376 \mathrm{MHz}, \mathrm{CDCl}_{3}\right)$ of $\mathbf{3 s a}$

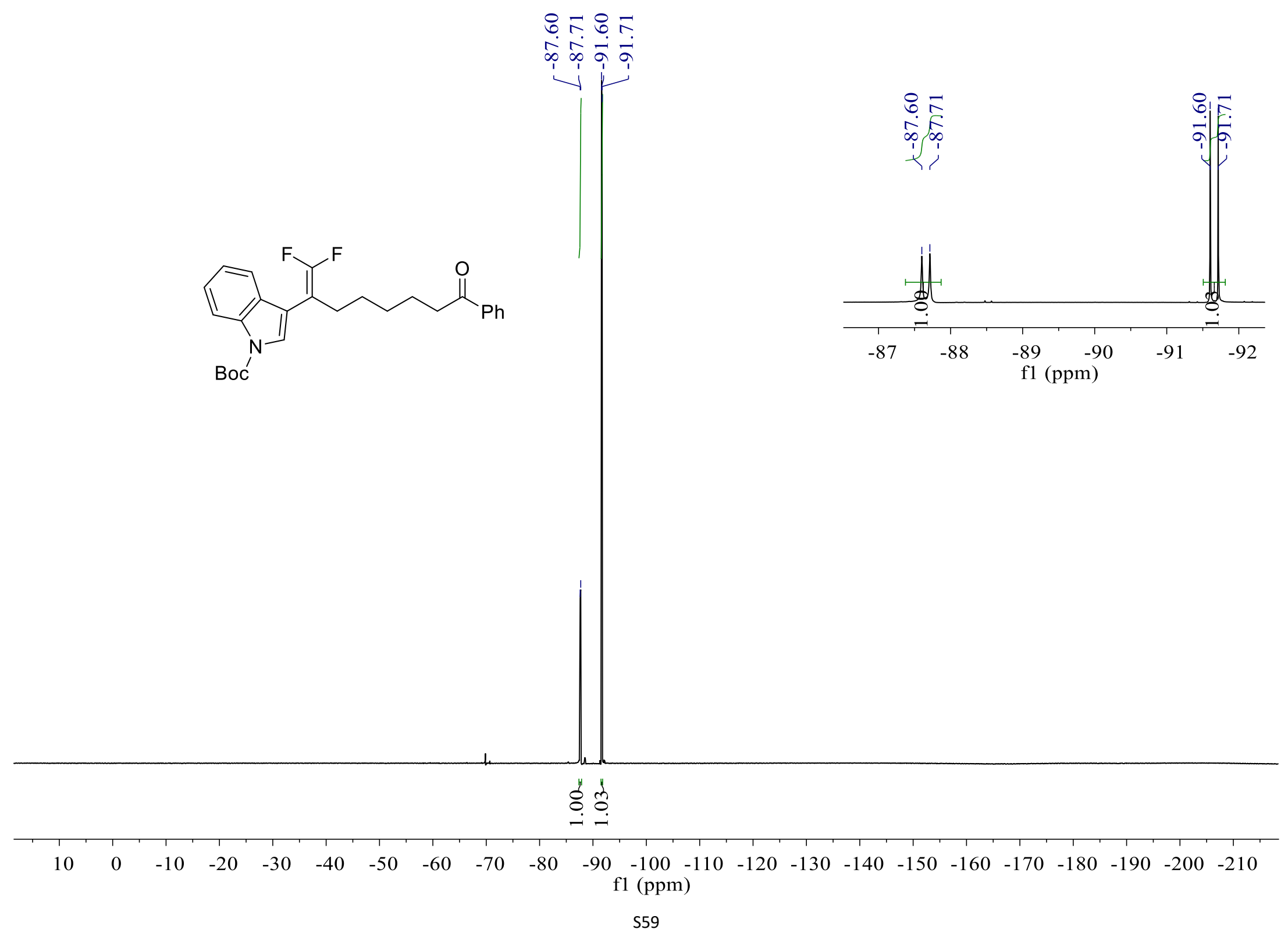




\section{${ }^{1} \mathrm{H}$ NMR-spectrum $\left(400 \mathrm{MHz}, \mathrm{CDCl}_{3}\right)$ of 3 ta}

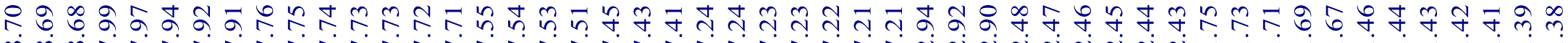

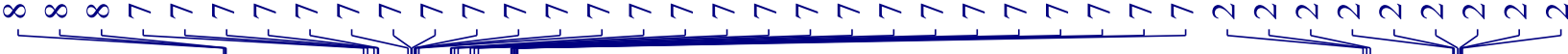

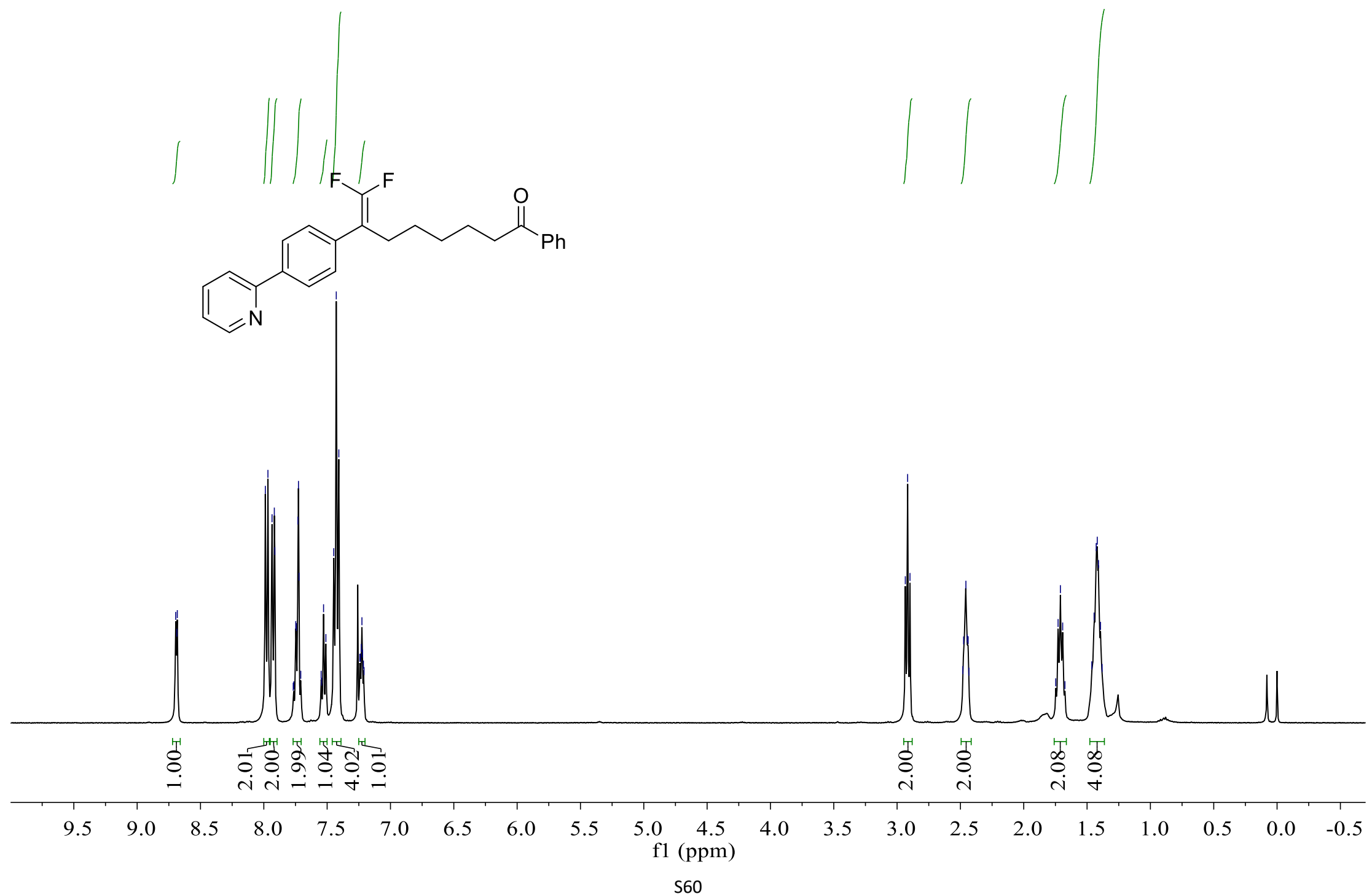


${ }^{13} \mathrm{C}\left\{{ }^{1} \mathrm{H}\right\}$ NMR-spectrum $\left(101 \mathrm{MHz}, \mathrm{CDCl}_{3}\right)$ of 3 ta

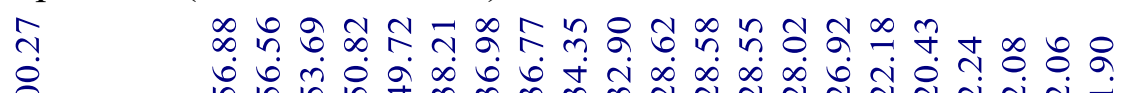

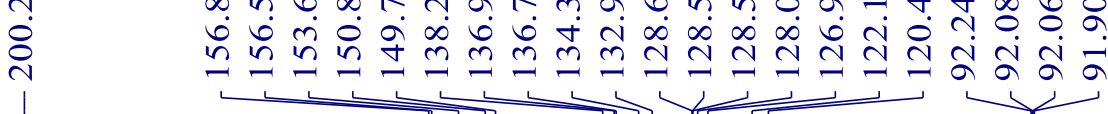

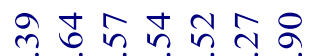

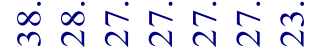

1
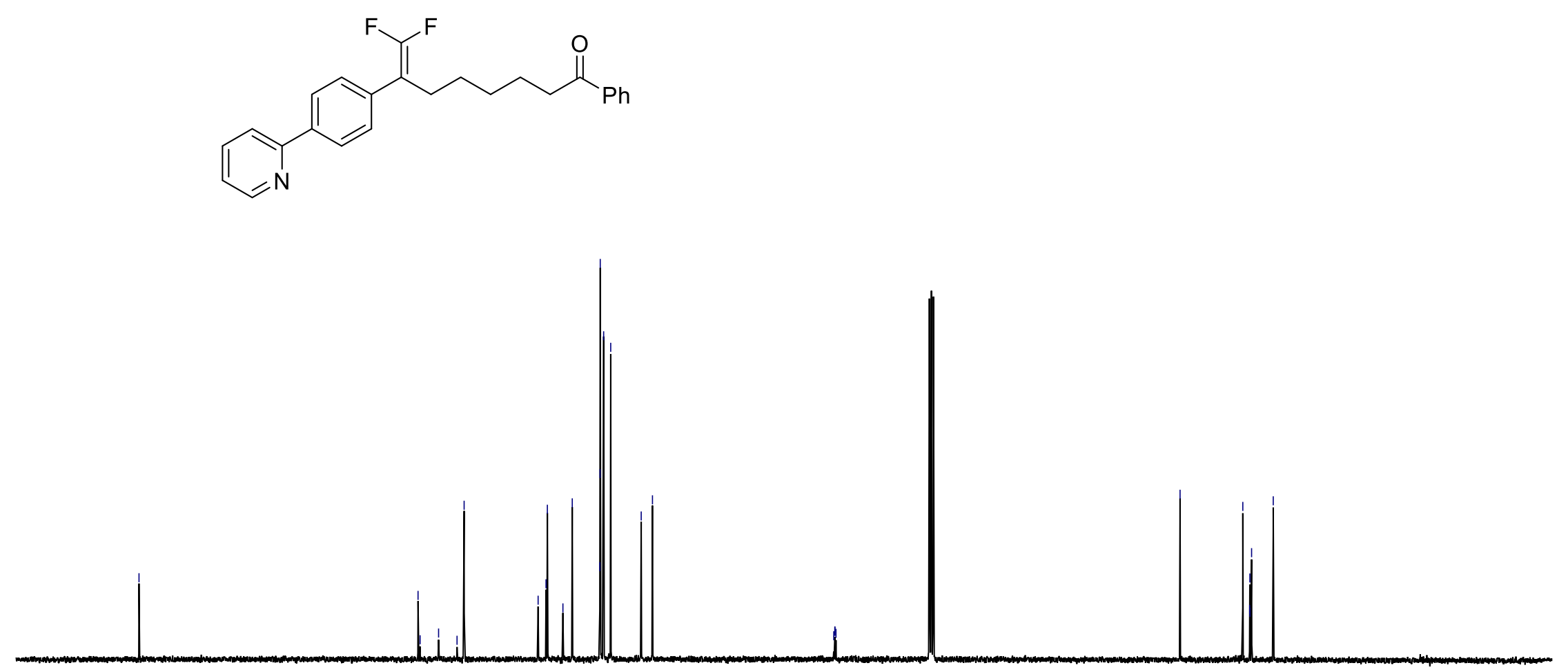

$\begin{array}{llllllllllll}210 & 200 & 190 & 180 & 170 & 160 & 150 & 140 & 130 & 120 & 110 & 100\end{array}$ f1 (ppm)

S61 
${ }^{19} \mathrm{~F}$ NMR-spectrum $\left(376 \mathrm{MHz}, \mathrm{CDCl}_{3}\right)$ of 3 ta

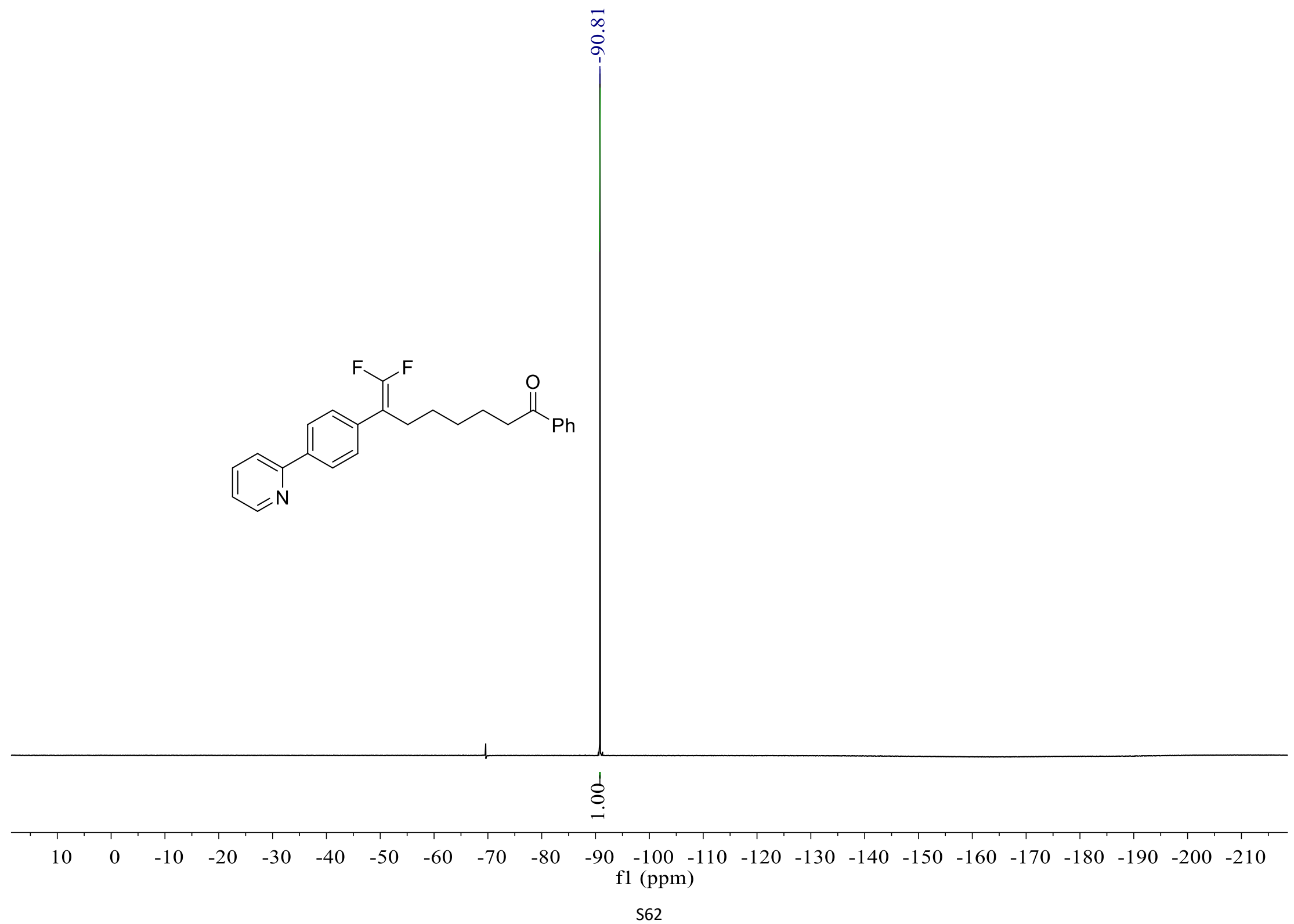




\section{${ }^{1} \mathrm{H}$ NMR-spectrum (400 MHz, $\mathrm{CDCl}_{3}$ ) of 3ua} के =

।
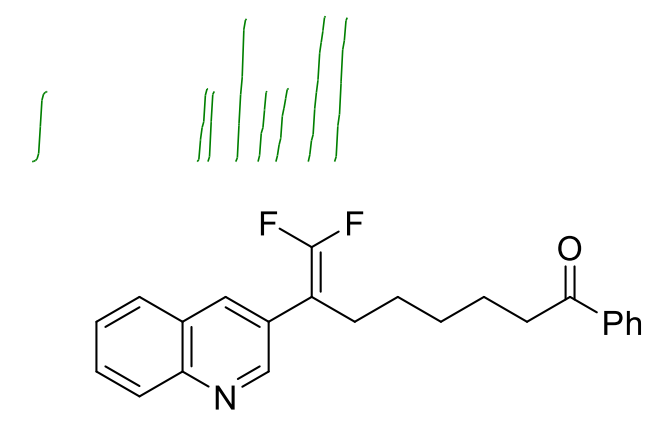

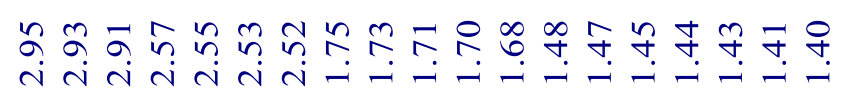
vivinju

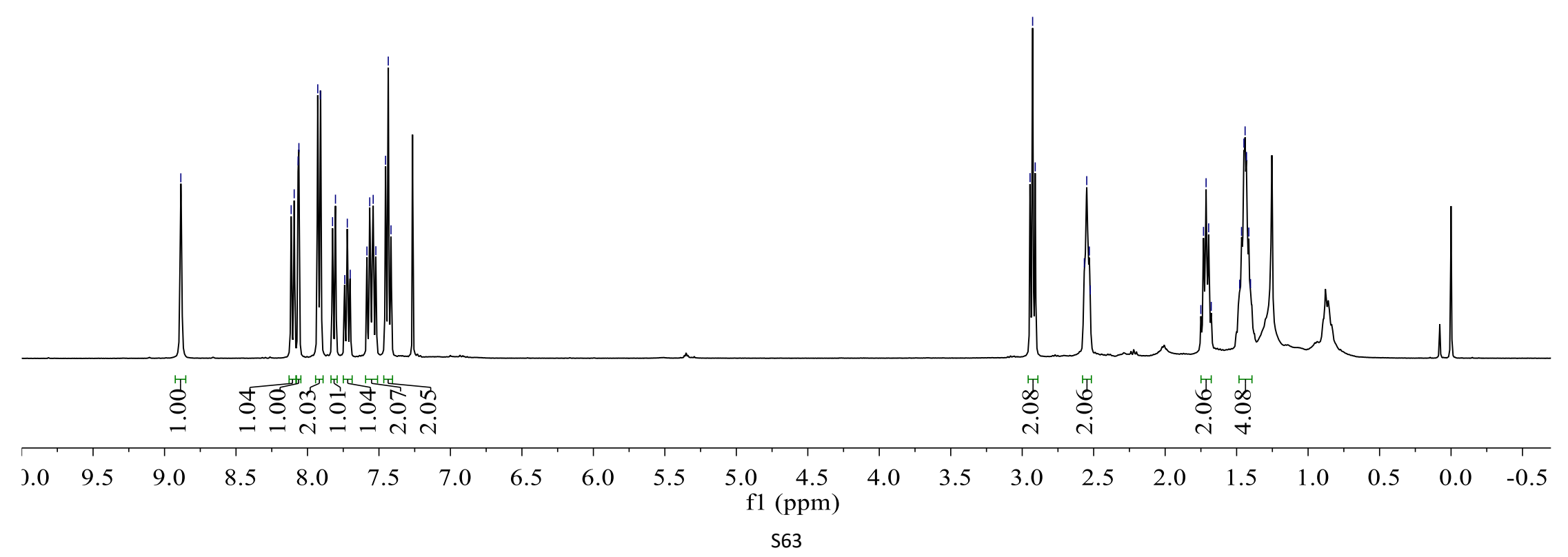


${ }^{13} \mathrm{C}\left\{{ }^{1} \mathrm{H}\right\}$ NMR-spectrum $\left(101 \mathrm{MHz}, \mathrm{CDCl}_{3}\right)$ of $\mathbf{3 u a}$

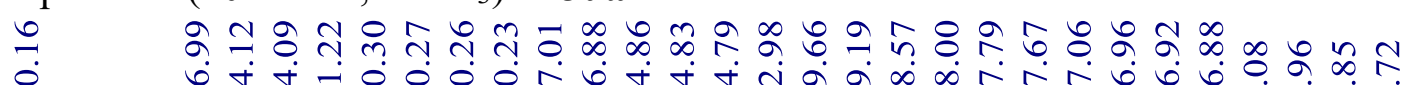

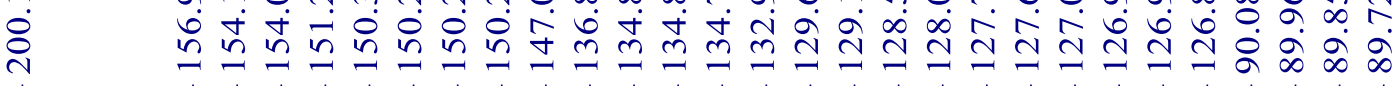

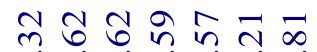

$\stackrel{\infty}{\infty} \stackrel{\infty}{\sim} \hat{\sim} \hat{\sim} \hat{\sim} \ddot{n}$
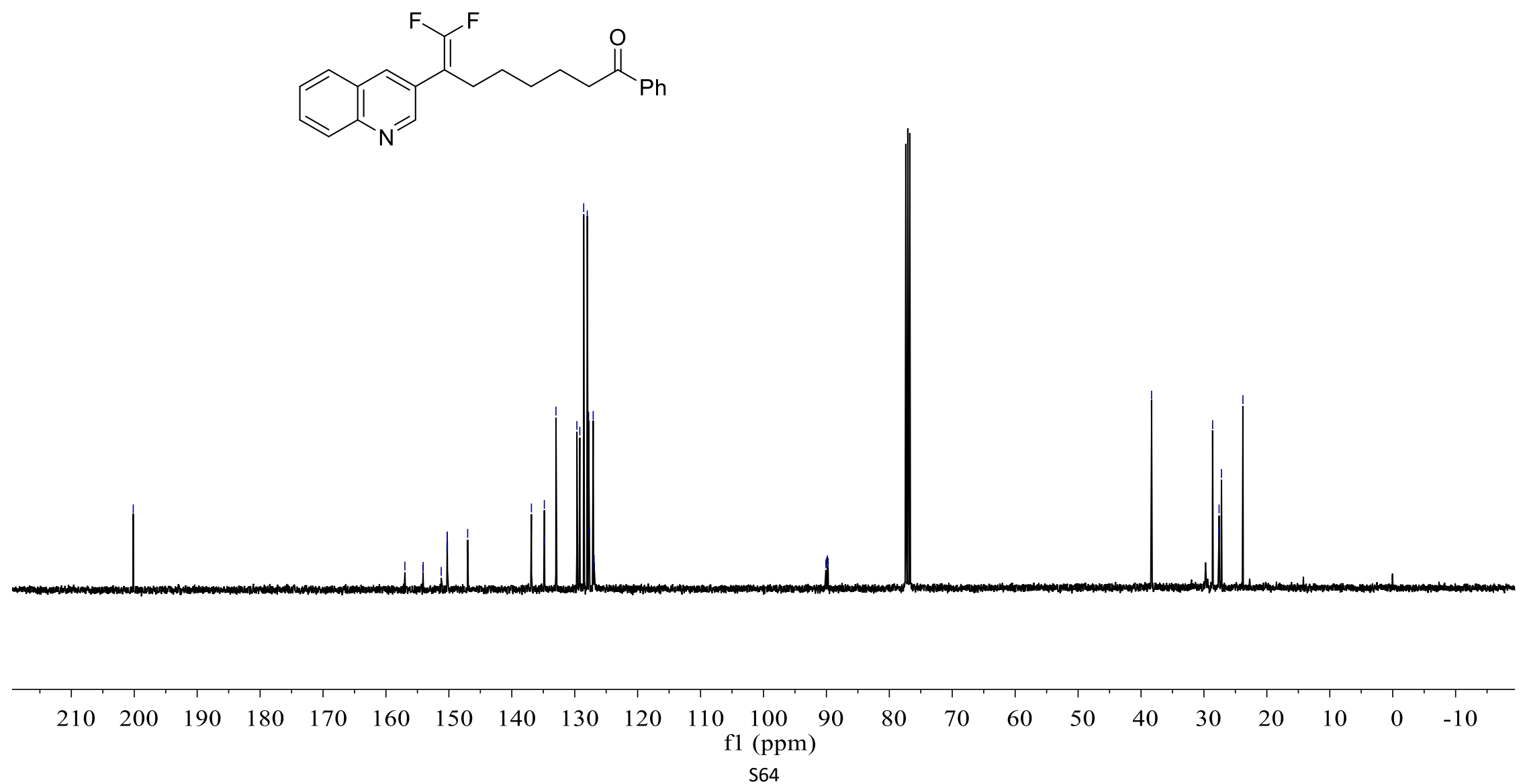
${ }^{19}$ F NMR-spectrum $\left(376 \mathrm{MHz}, \mathrm{CDCl}_{3}\right)$ of 3 ua

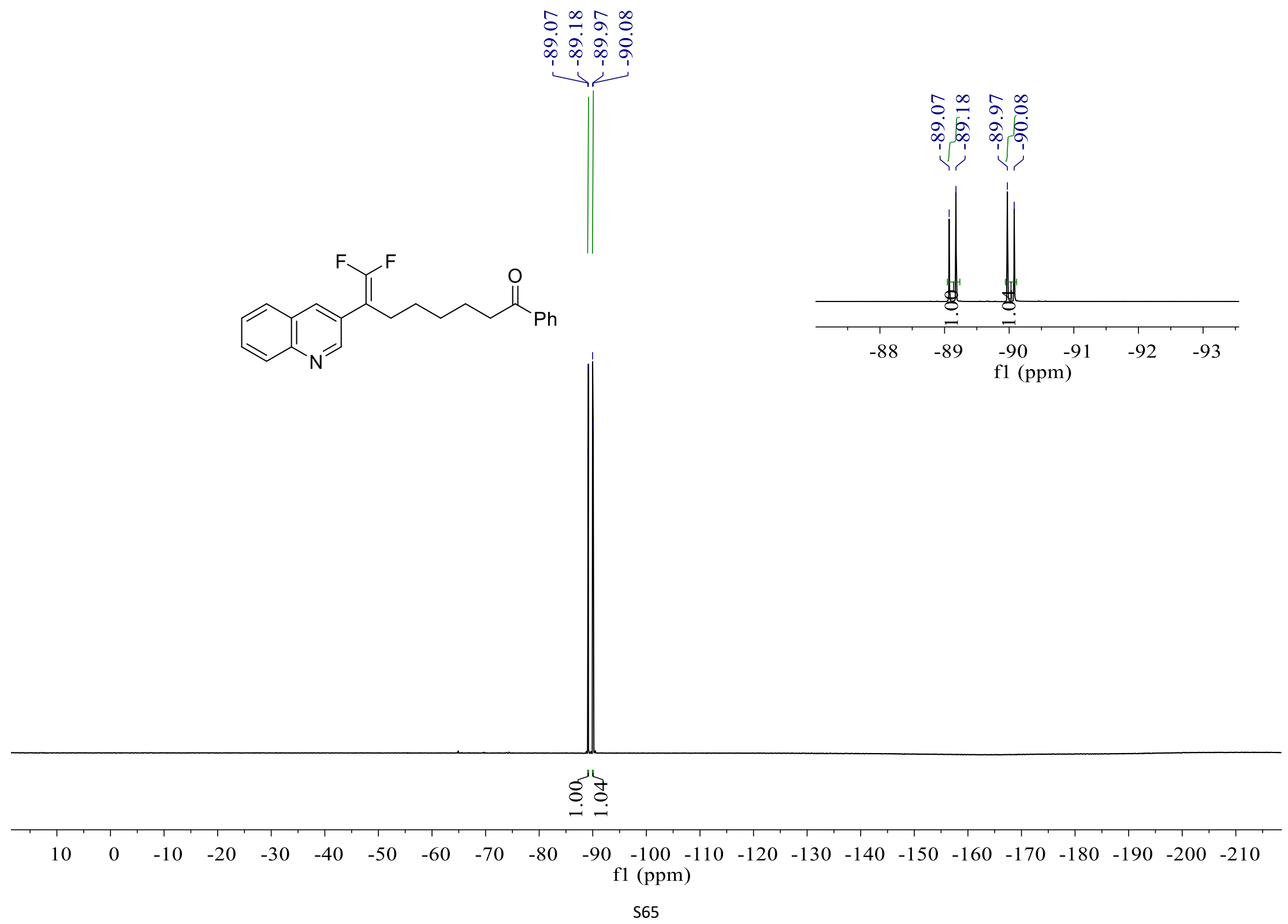




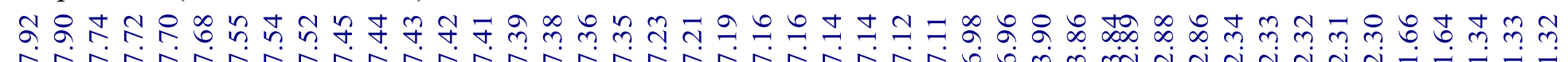

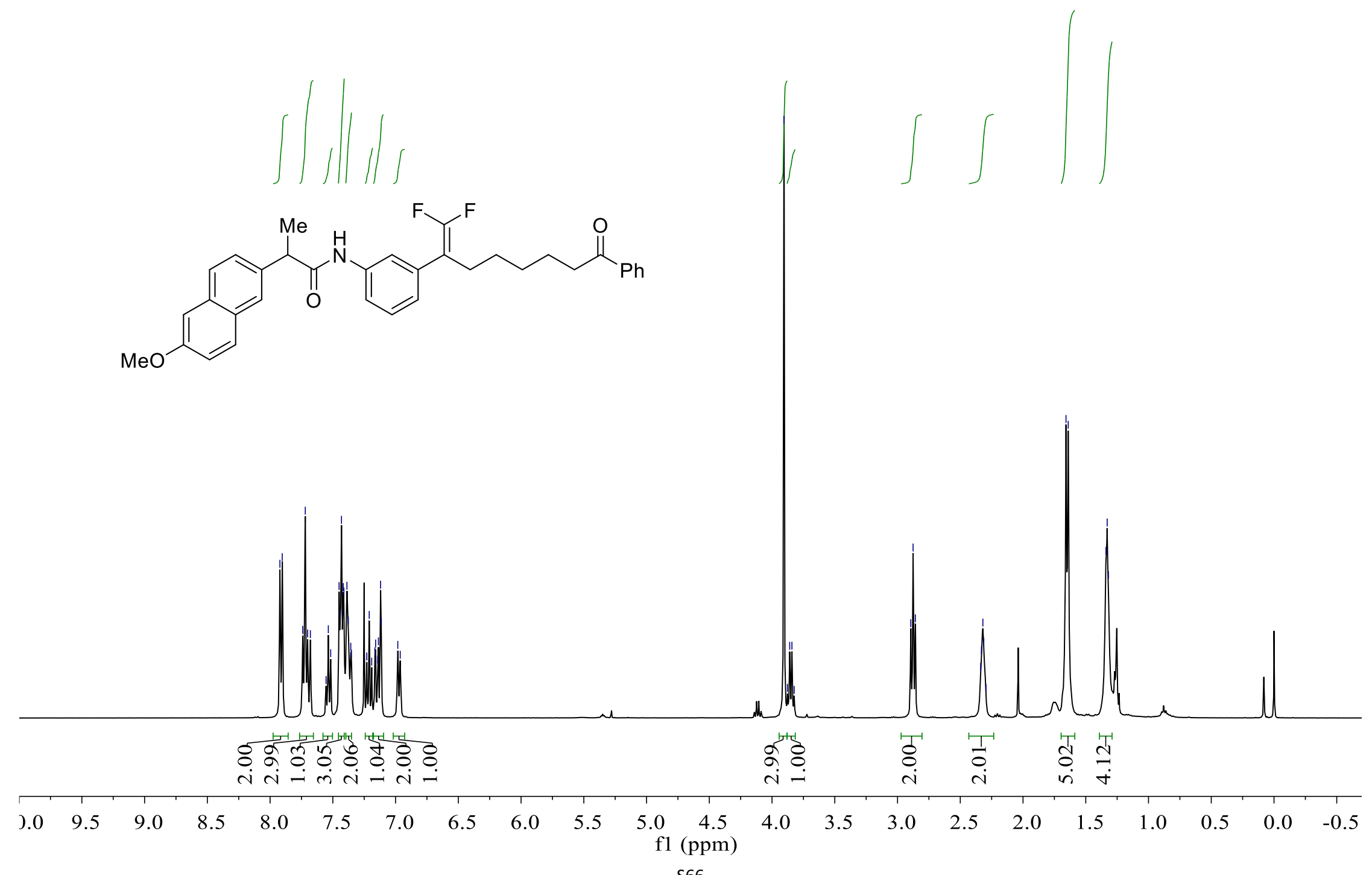


${ }^{13} \mathrm{C}\left\{{ }^{1} \mathrm{H}\right\}$ NMR-spectrum $\left(126 \mathrm{MHz}, \mathrm{CDCl}_{3}\right)$ of 3va

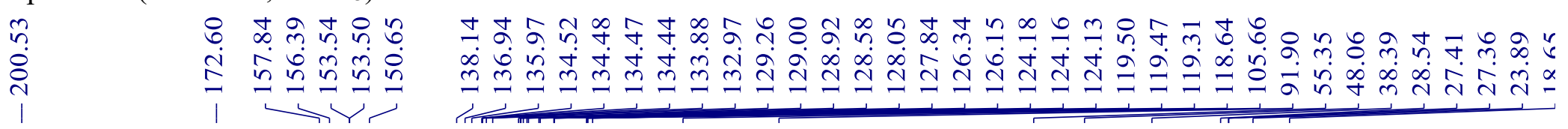

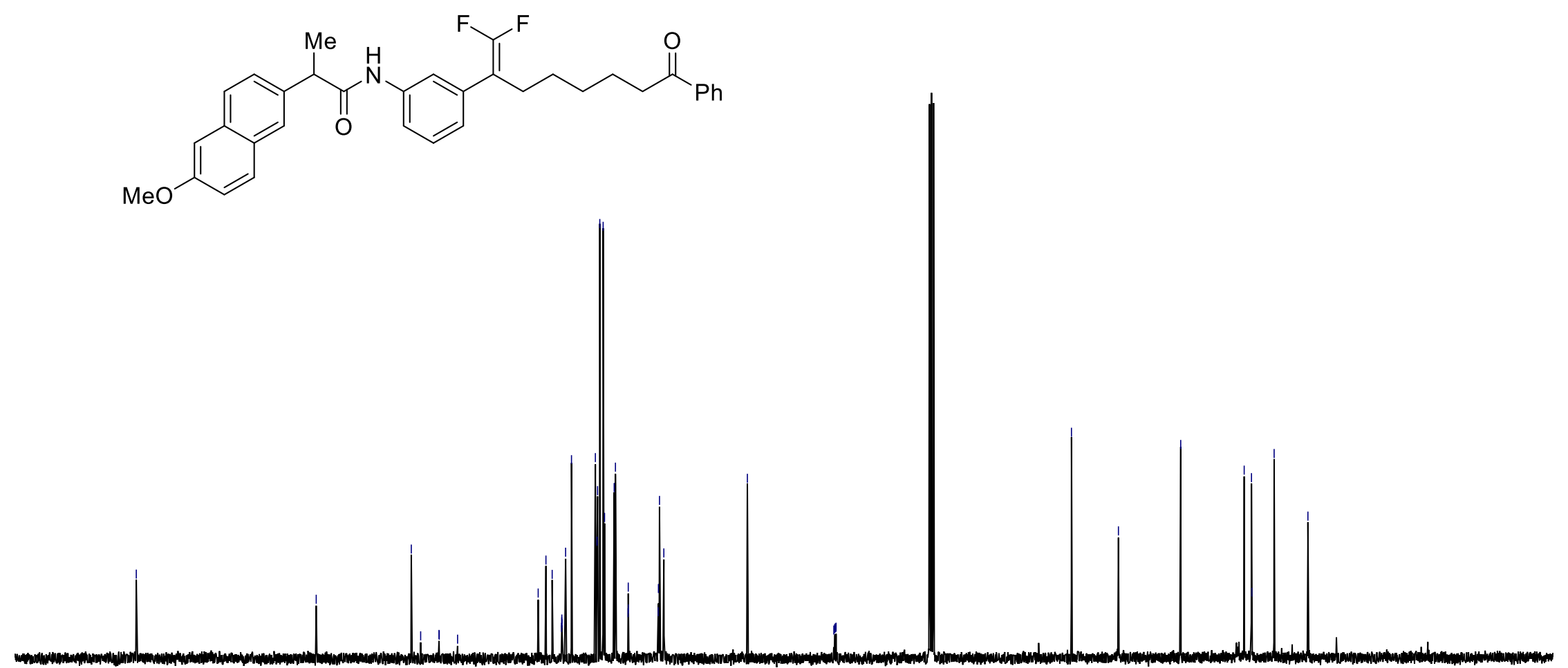

$\begin{array}{lllllllllllllllllllllll}210 & 200 & 190 & 180 & 170 & 160 & 150 & 140 & 130 & 120 & 110 \begin{array}{c}100 \\ \mathrm{f1}(\mathrm{ppm})\end{array} & 90 & 80 & 70 & 60 & 50 & 40 & 30 & 20 & 10 & 0 & -10\end{array}$

S67 
${ }^{13} \mathrm{~F}$ NMR-spectrum $\left(376 \mathrm{MHz}, \mathrm{CDCl}_{3}\right)$ of $\mathbf{3 v a}$

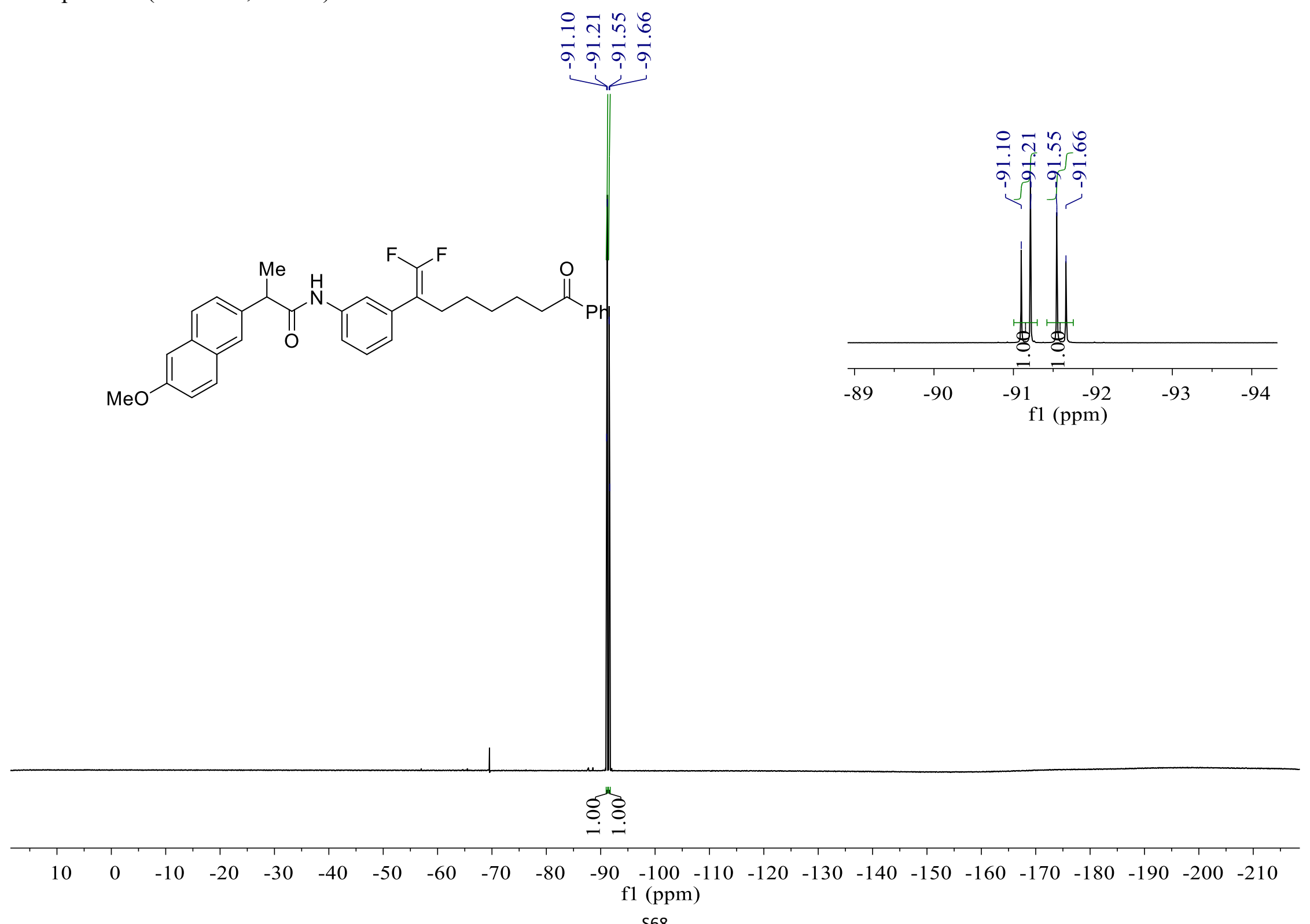
$1(\mathrm{ppm})$ 


\section{${ }^{1} \mathrm{H}$ NMR-spectrum $\left(500 \mathrm{MHz}, \mathrm{CDCl}_{3}\right)$ of $\mathbf{3 w a}$}

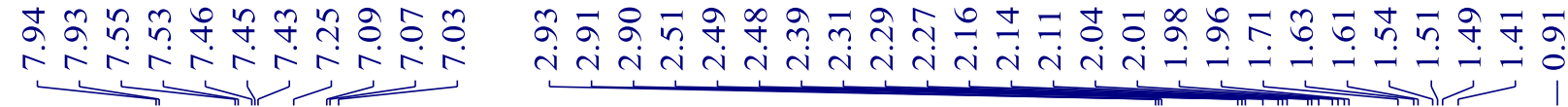

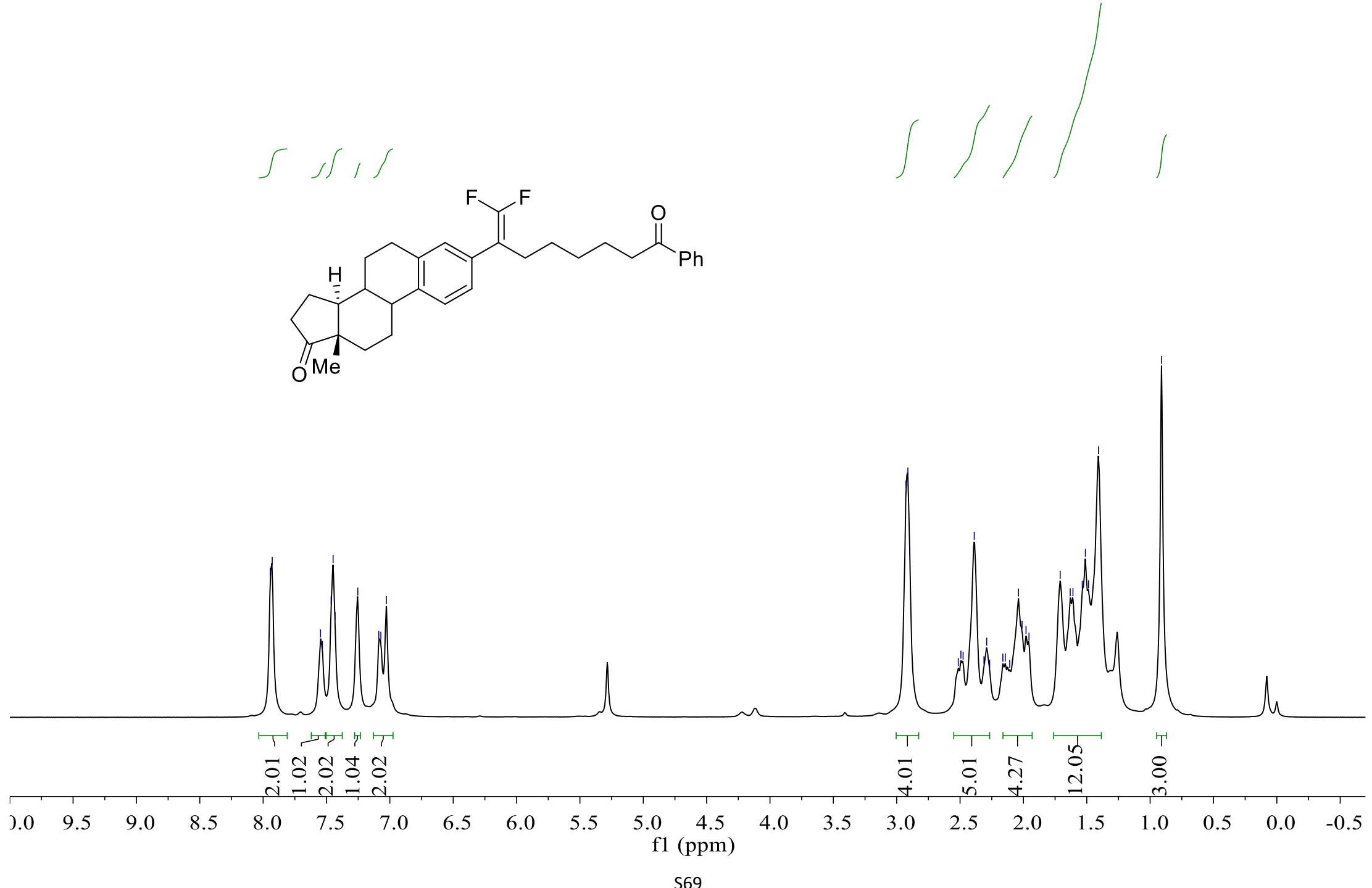

s69 
${ }^{13} \mathrm{C}\left\{{ }^{1} \mathrm{H}\right\}$ NMR-spectrum $\left(101 \mathrm{MHz}, \mathrm{CDCl}_{3}\right)$ of $\mathbf{3 w a}$

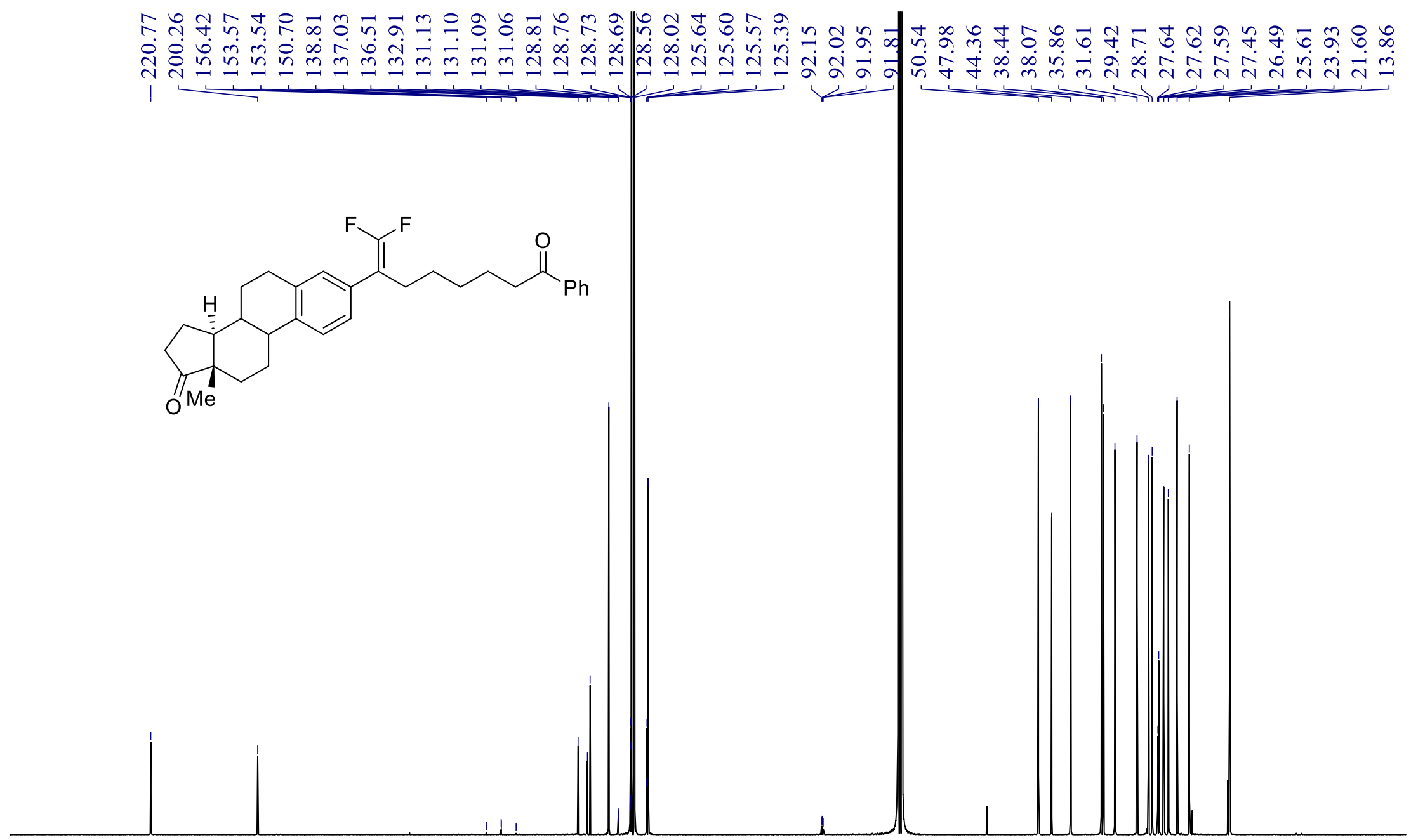

$\begin{array}{llllllllllllllllllllllllllllll}240 & 230 & 220 & 210 & 200 & 190 & 180 & 170 & 160 & 150 & 140 & 130 & 120 & 110 & 100 & 90 & 80 & 70 & 60 & 50 & 40 & 30 & 20 & 10 & 0 & -10\end{array}$ f1 (ppm) 
${ }^{19} \mathrm{~F}$ NMR-spectrum $\left(376 \mathrm{MHz}, \mathrm{CDCl}_{3}\right)$ of 3wa

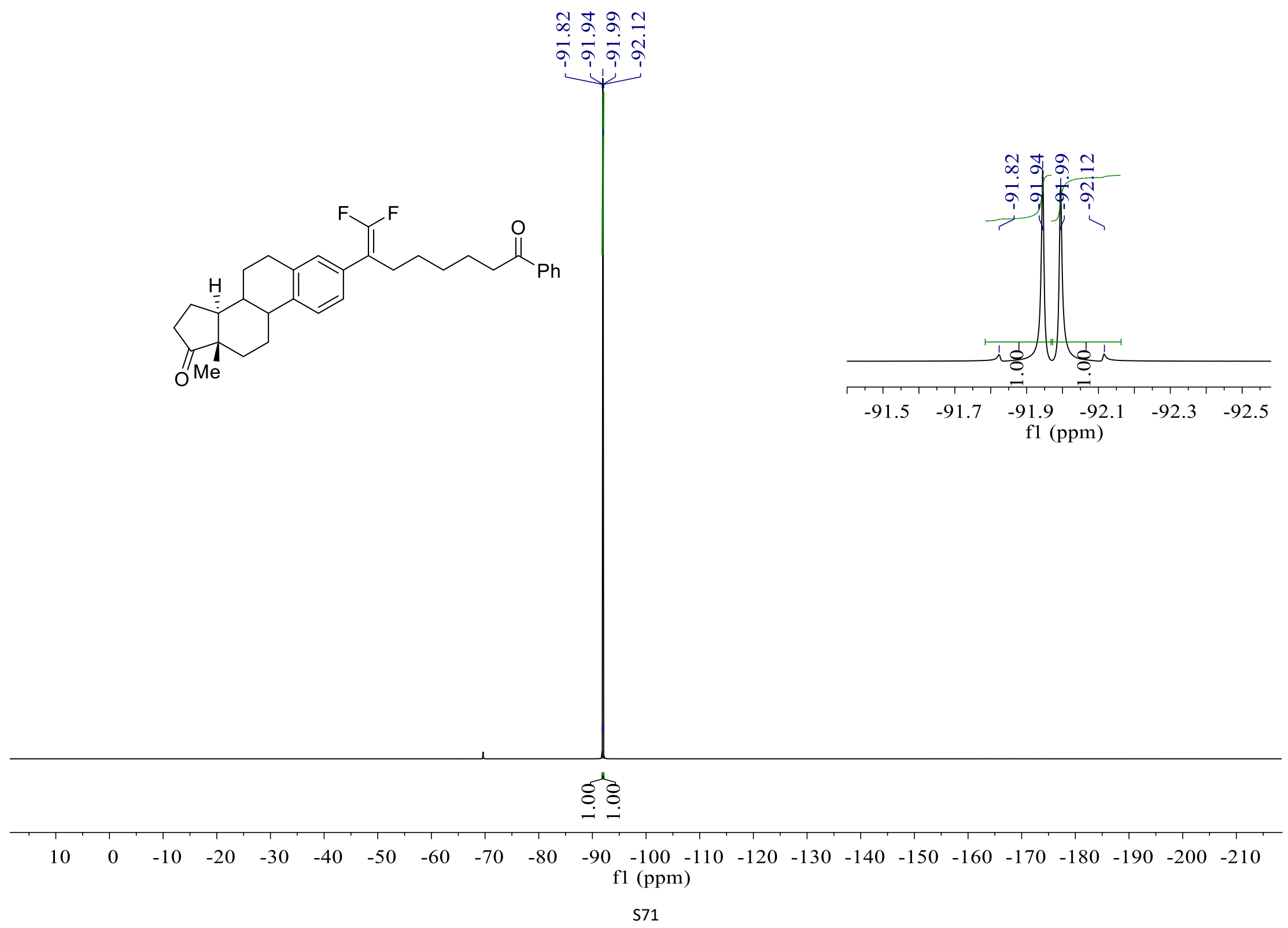




\section{${ }^{1} \mathrm{H}$ NMR-spectrum $\left(500 \mathrm{MHz}, \mathrm{CDCl}_{3}\right)$ of $\mathbf{3 a b}$}

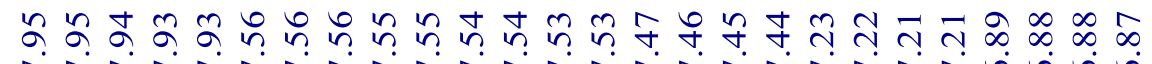

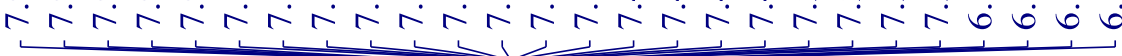

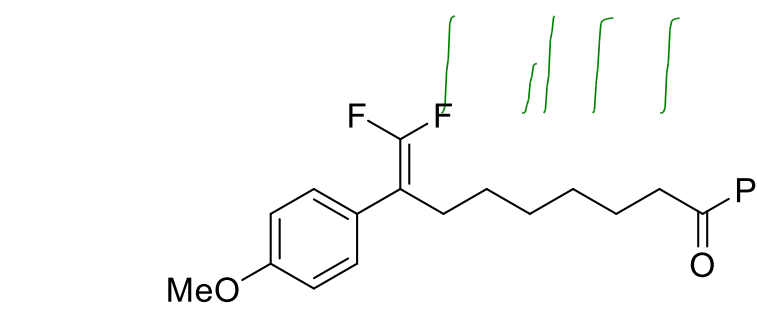

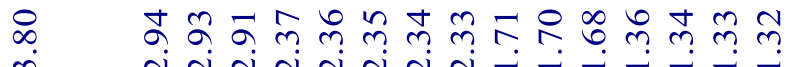

$\sqrt{4} \sqrt{4}$
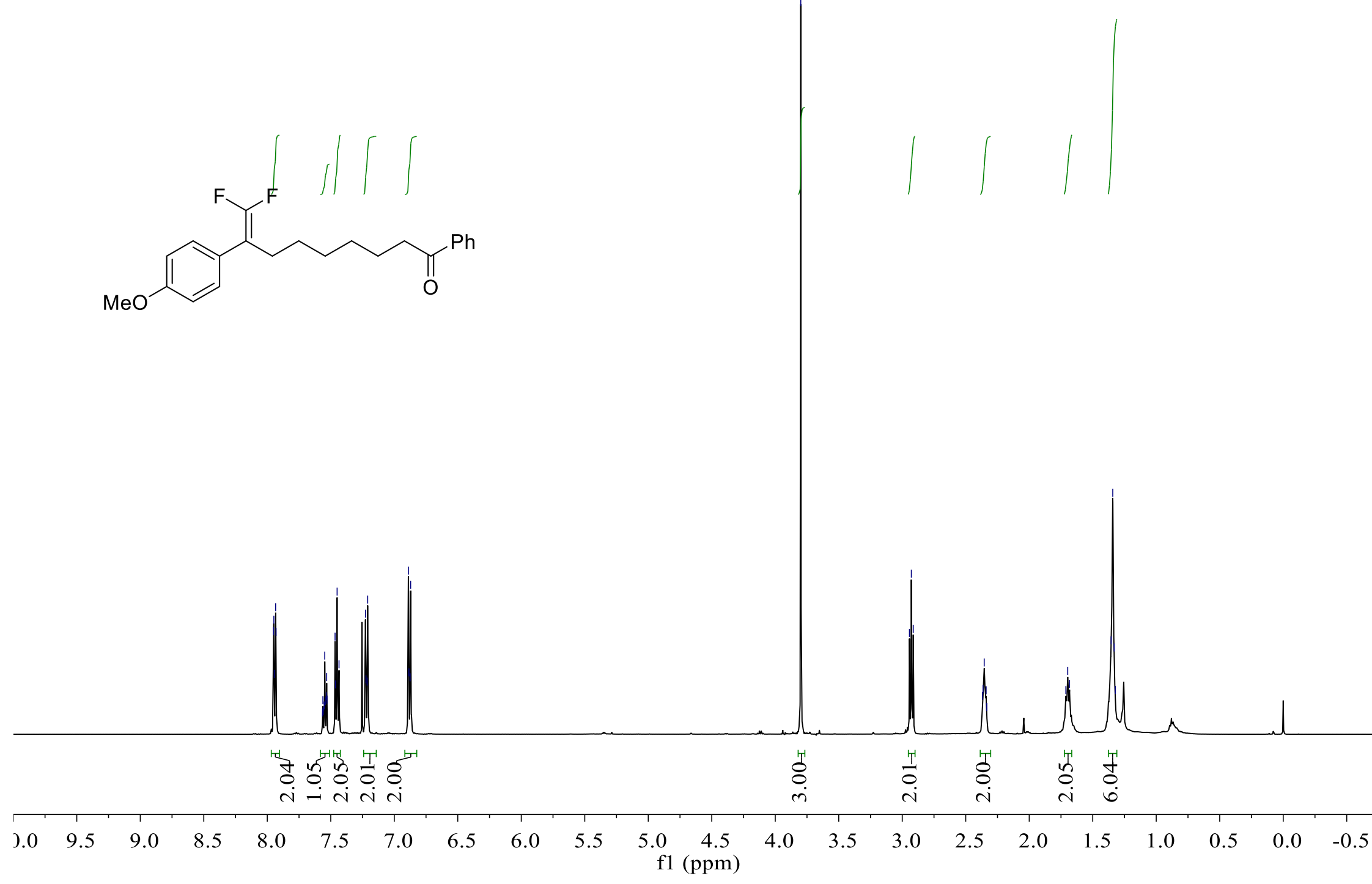

S72 
${ }^{13} \mathrm{C}\left\{{ }^{1} \mathrm{H}\right\}$ NMR-spectrum $\left(126 \mathrm{MHz}, \mathrm{CDCl}_{3}\right)$ of $\mathbf{3 a b}$

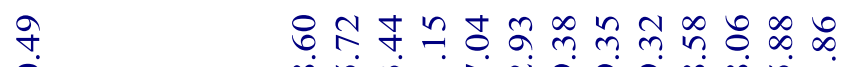

$\stackrel{8}{\stackrel{2}{1}}$

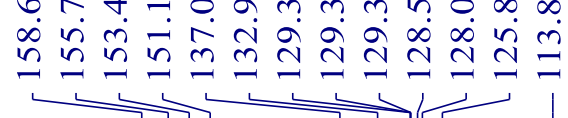

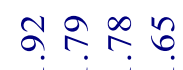

बंबेंब

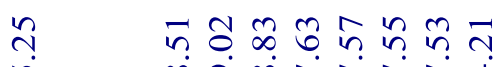

in

m तेंत्रत

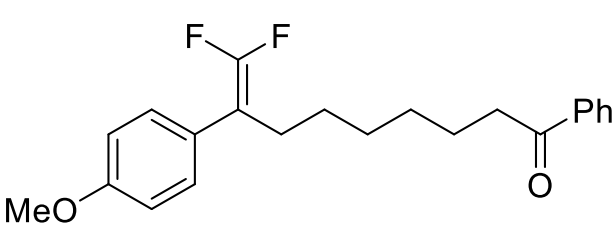

$\mathrm{MeO}$

O
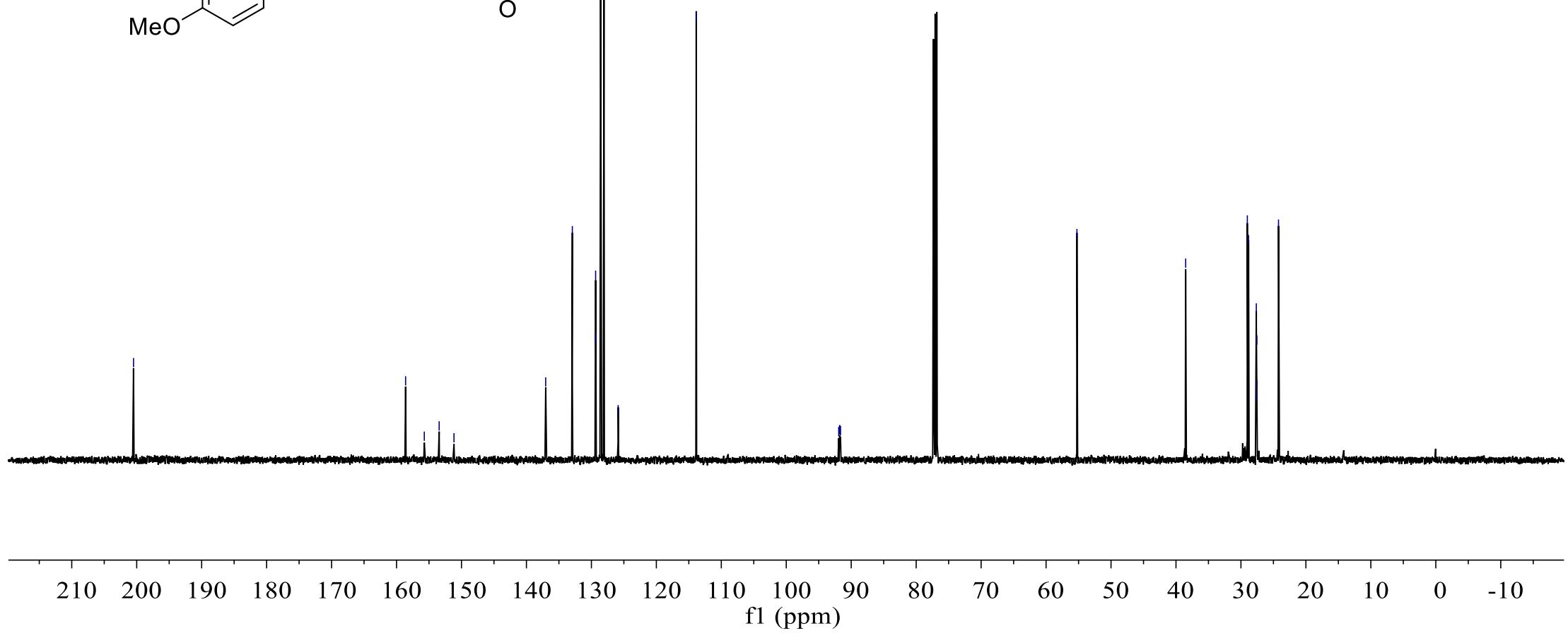

S73 
${ }^{19} \mathrm{~F}$ NMR-spectrum $\left(471 \mathrm{MHz}, \mathrm{CDCl}_{3}\right)$ of $\mathbf{3 a b}$

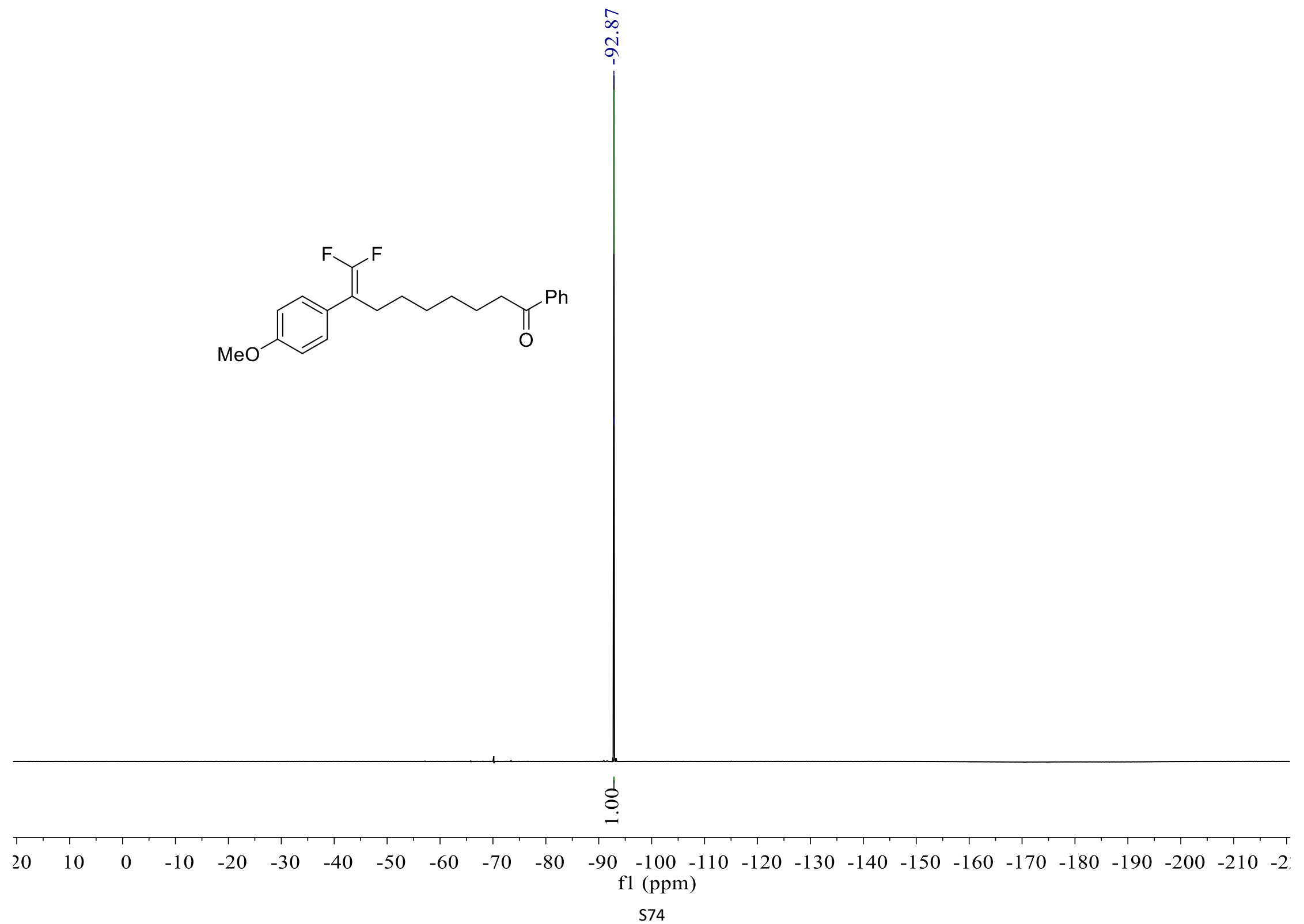




\section{${ }^{1} \mathrm{H}$ NMR-spectrum $\left(400 \mathrm{MHz}, \mathrm{CDCl}_{3}\right)$ of $\mathbf{3 a c}$}

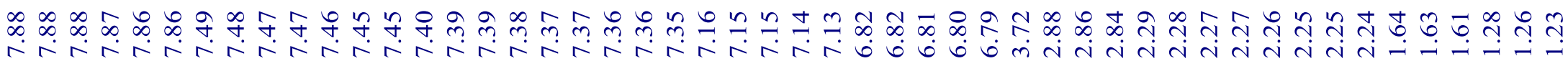

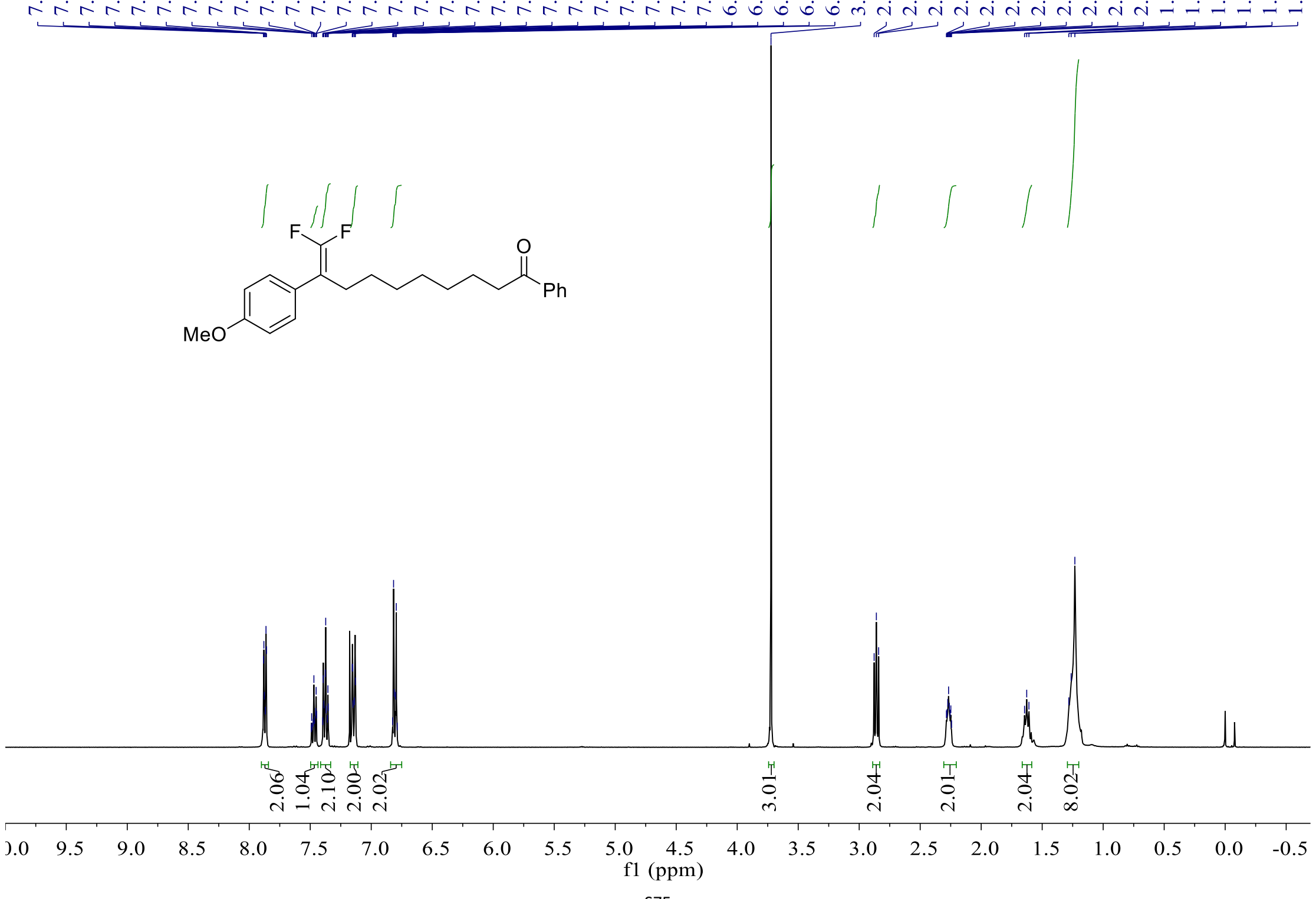

S75 
${ }^{13} \mathrm{C}\left\{{ }^{1} \mathrm{H}\right\}$ NMR-spectrum $\left(101 \mathrm{MHz}, \mathrm{CDCl}_{3}\right)$ of $\mathbf{3 a c}$

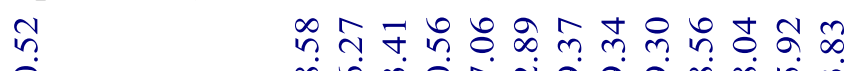

iे

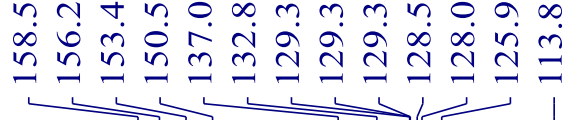

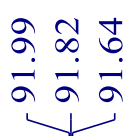

กิ

n

1

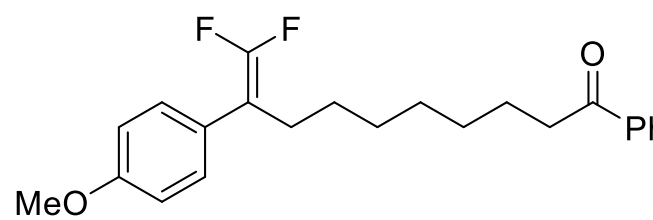

$210 \quad 200$

$200 \quad 190$

$\begin{array}{lll}180 & 170 \quad 160\end{array}$

$\begin{array}{lll}150 & 140 & 130\end{array}$

$\begin{array}{llll}20 & 110 & 100 \quad 90\end{array}$

f1 $(\mathrm{ppm})$

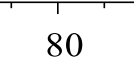

$70 \quad 60$

$60 \quad 50$

S76 
${ }^{19} \mathrm{~F}$ NMR-spectrum $\left(376 \mathrm{MHz}, \mathrm{CDCl}_{3}\right)$ of $\mathbf{3 a c}$

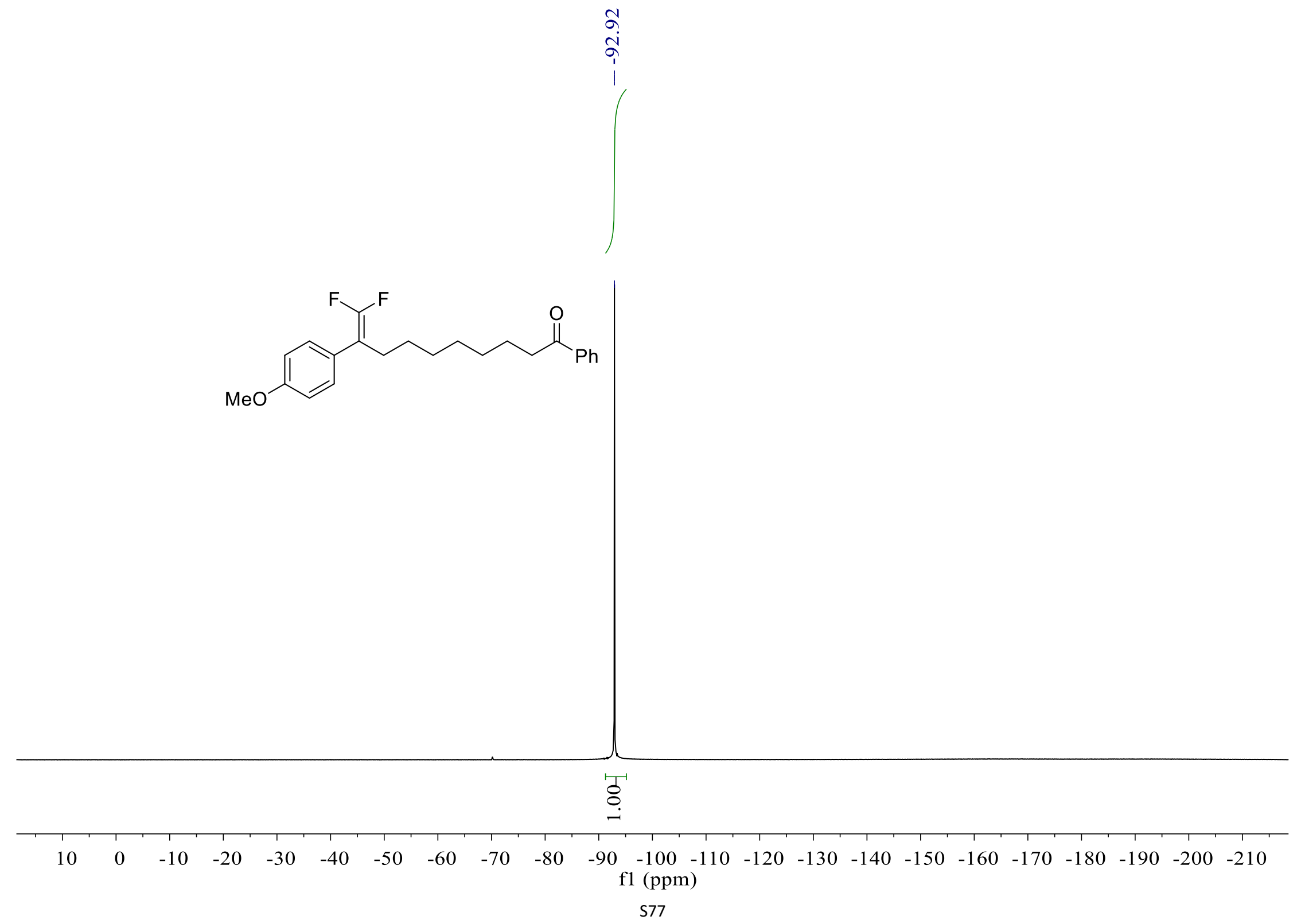




\section{${ }^{1} \mathrm{H}$ NMR-spectrum $\left(400 \mathrm{MHz}, \mathrm{CDCl}_{3}\right)$ of $\mathbf{3 a d}$}

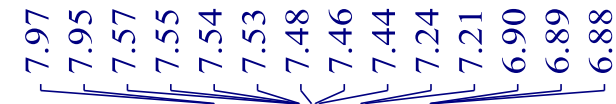

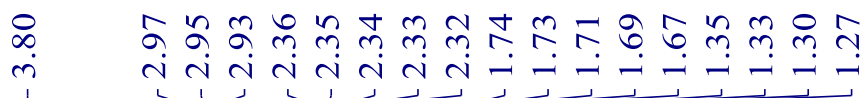

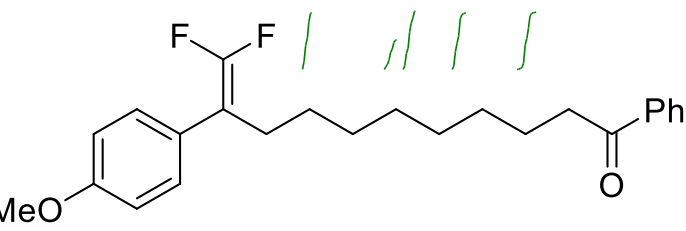

in

$\mathrm{MeO}$
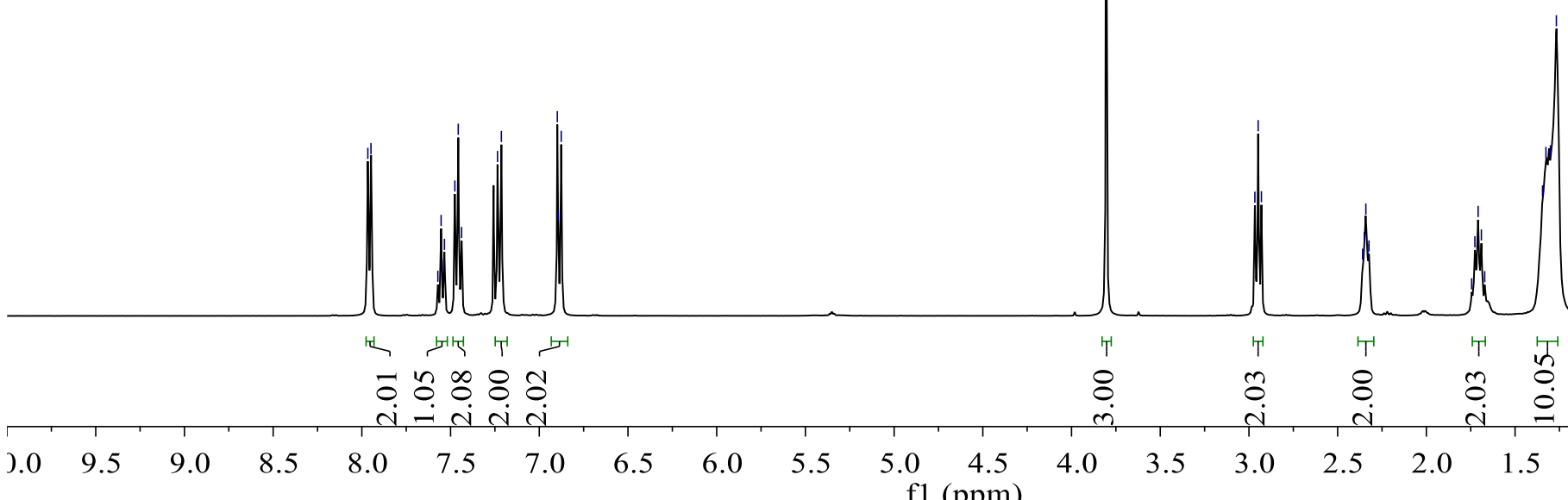
${ }^{13} \mathrm{C}\left\{{ }^{1} \mathrm{H}\right\}$ NMR-spectrum (101 MHz, $\left.\mathrm{CDCl}_{3}\right)$ of $\mathbf{3 a d}$

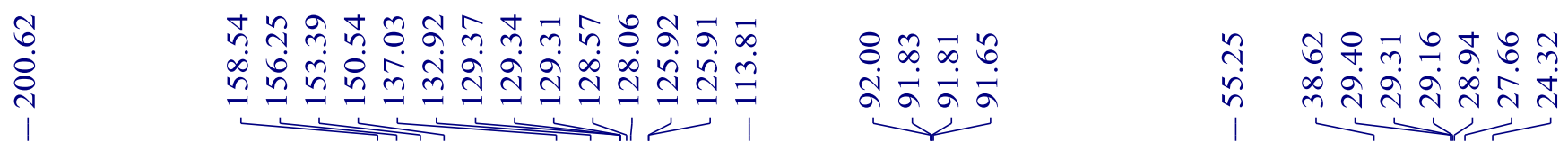<smiles>COc1ccc(C(CCCCCCCCC(=O)c2ccccc2)=C(F)F)cc1</smiles>

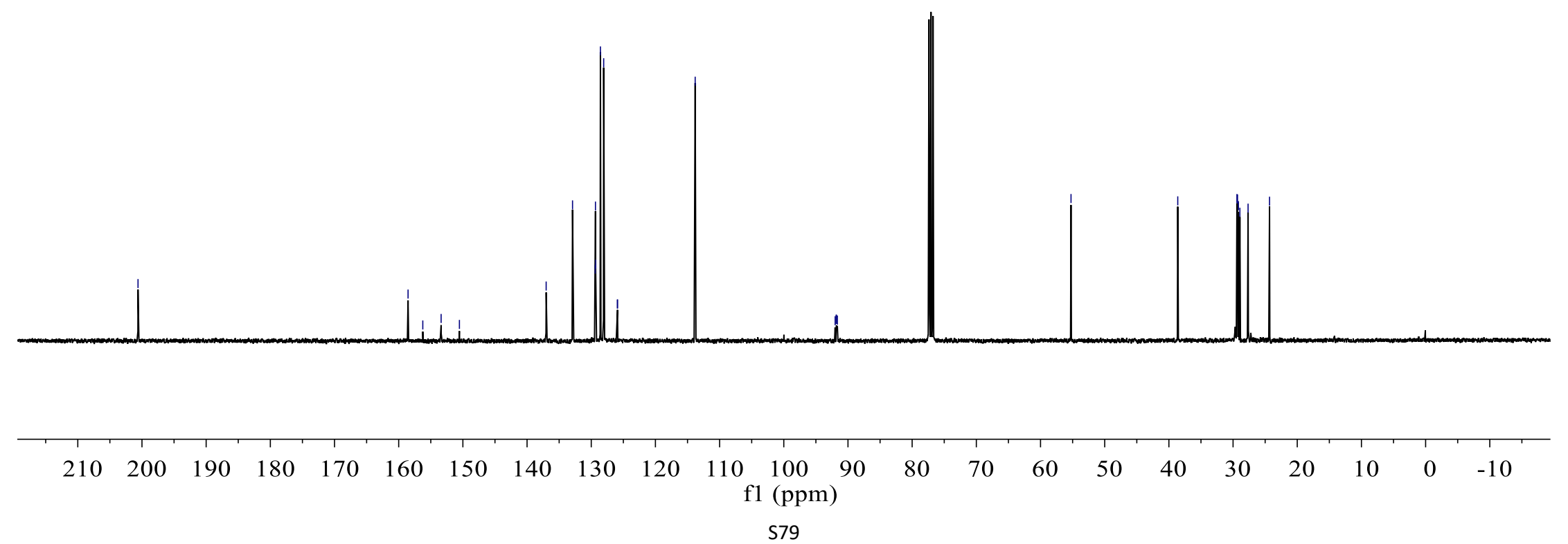


${ }^{19}$ F NMR-spectrum (376 MHz, $\mathrm{CDCl}_{3}$ ) of $\mathbf{3 a d}$

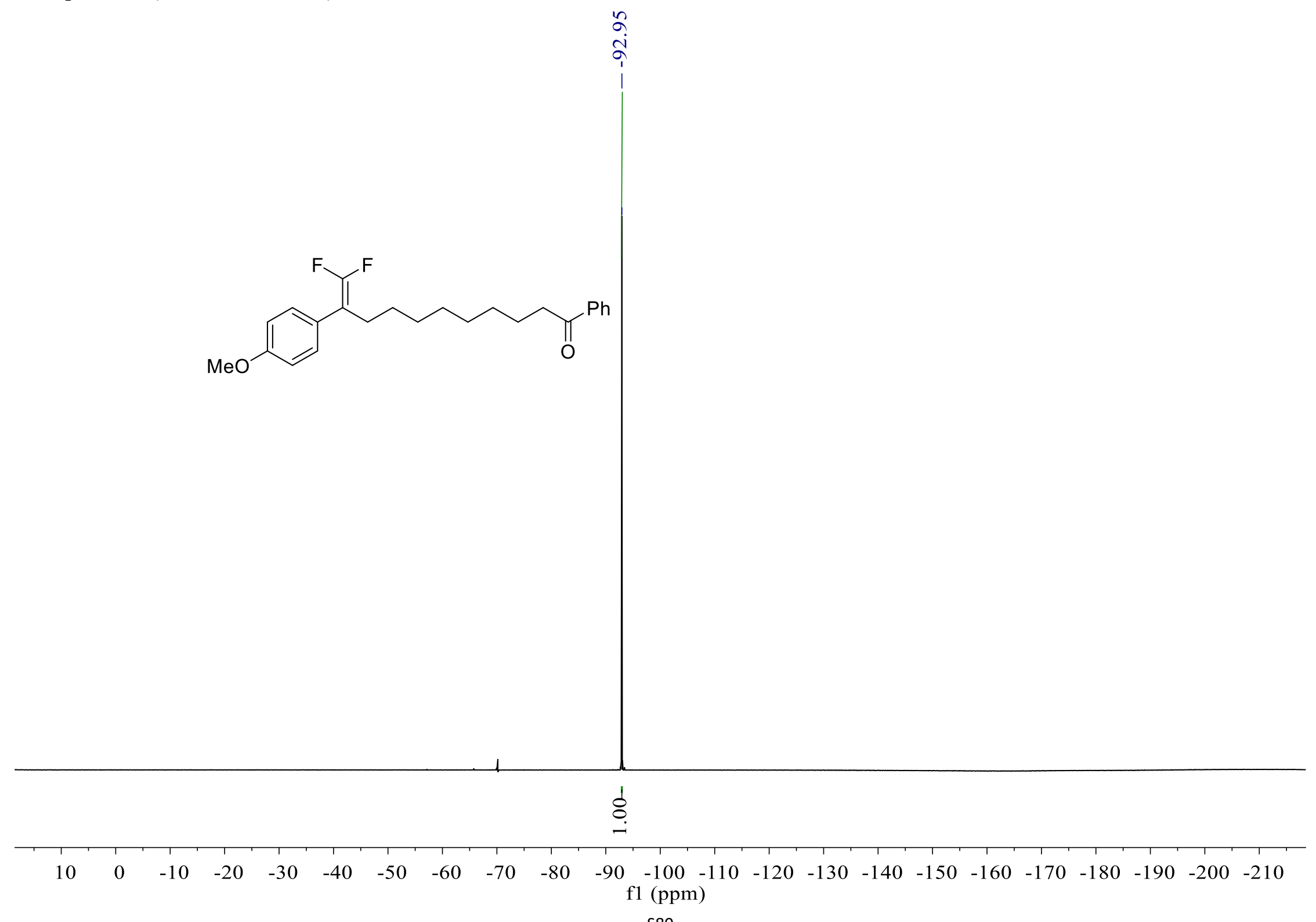

S80 


\section{${ }^{1} \mathrm{H}$ NMR-spectrum $\left(400 \mathrm{MHz}, \mathrm{CDCl}_{3}\right)$ of $\mathbf{3 a e}$}

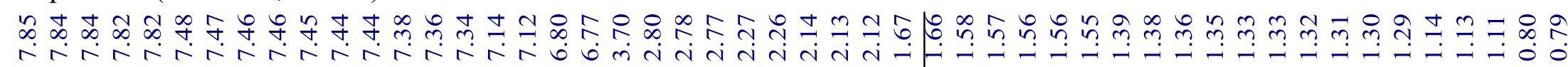

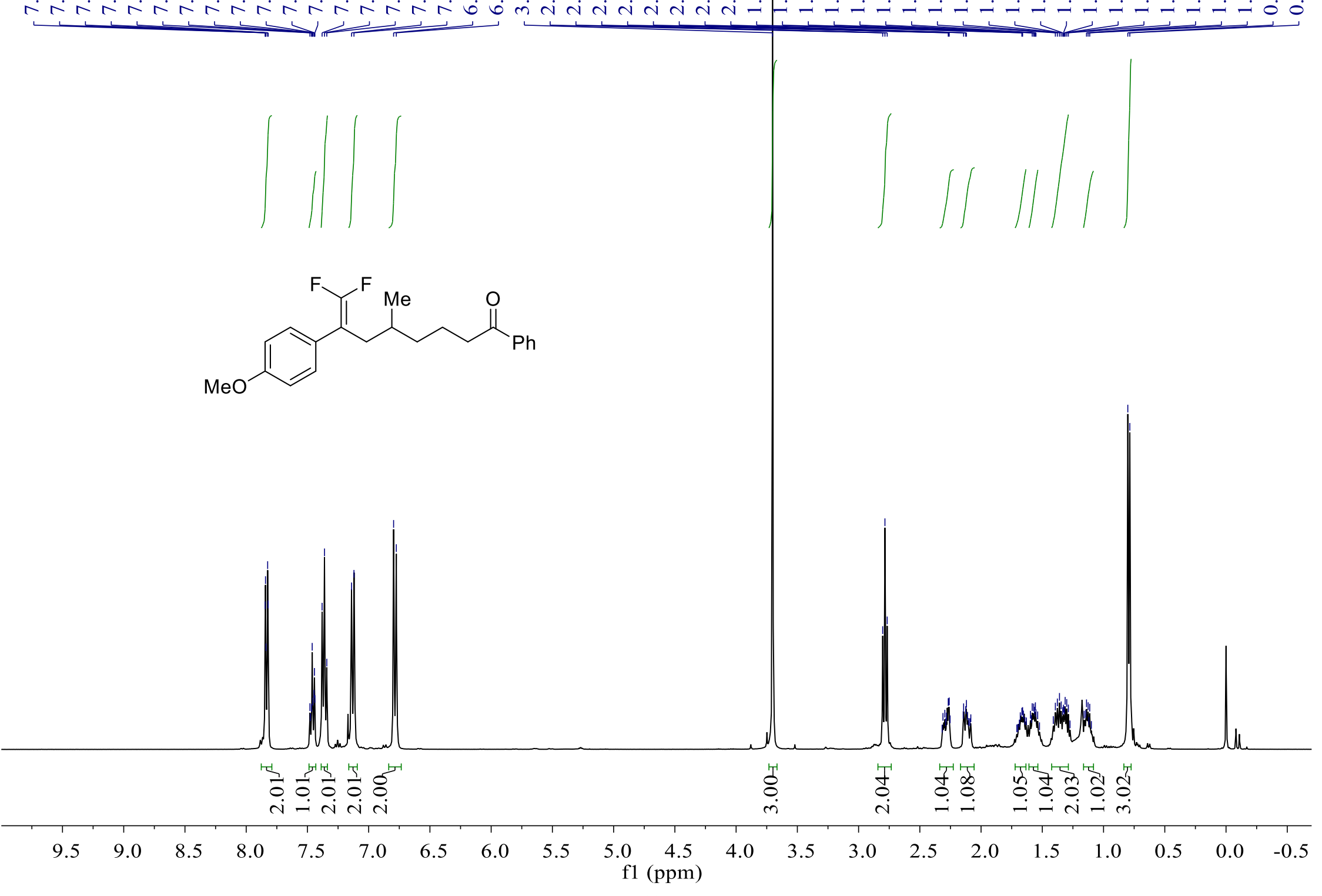

$\mathrm{s} 81$ 
${ }^{13} \mathrm{C}\left\{{ }^{1} \mathrm{H}\right\}$ NMR-spectrum (101 MHz, $\left.\mathrm{CDCl}_{3}\right)$ of 3ae

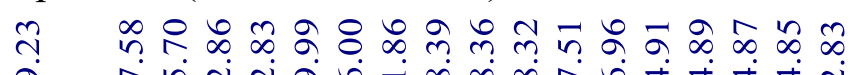

a $\quad$ in

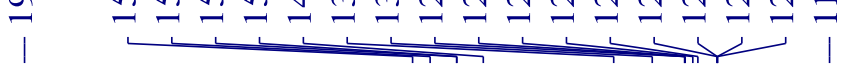

$\hat{\alpha} \infty \hat{\infty} \leqslant$

के

事

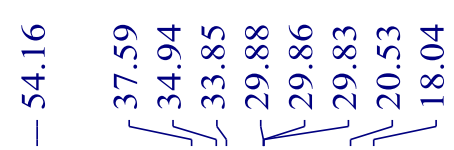

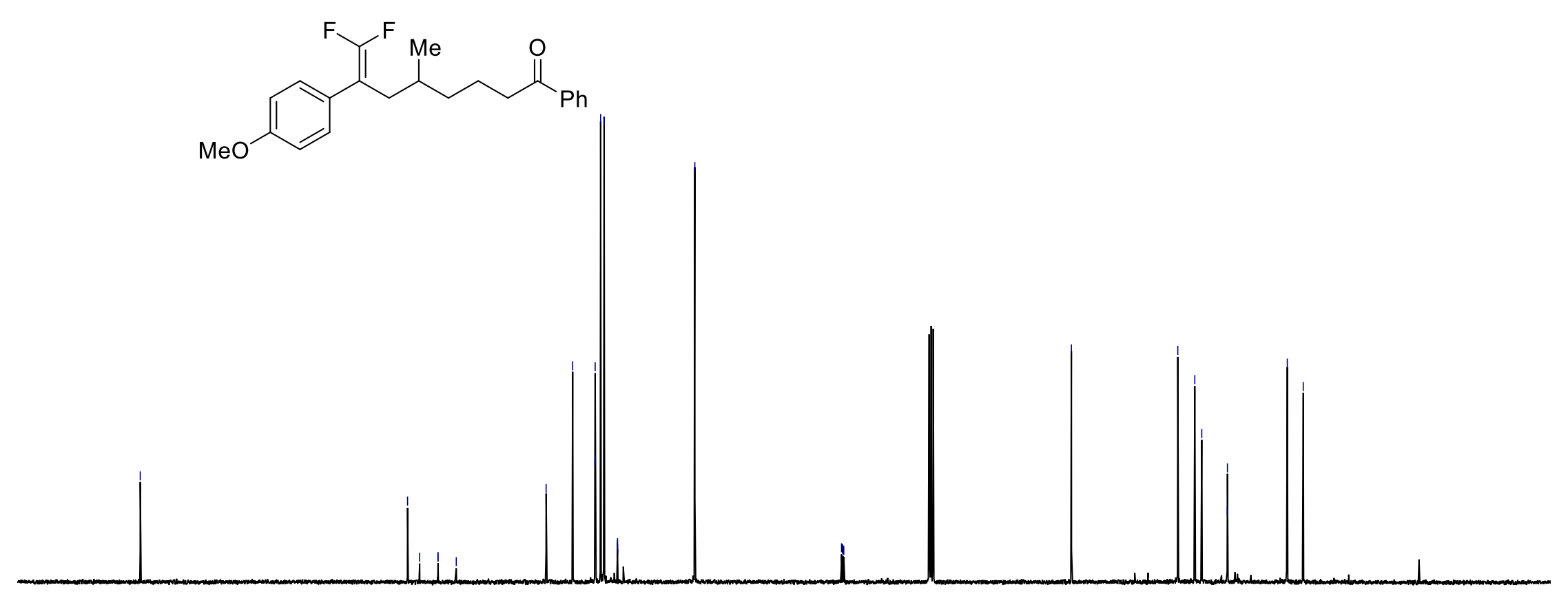

$\begin{array}{llllllllllllllllllllllll}210 & 200 & 190 & 180 & 170 & 160 & 150 & 140 & 130 & 120 & 110 & 100 & 90 & 80 & 70 & 60 & 50 & 40 & 30 & 20 & 10 & 0 & -10 & -\hat{2}\end{array}$ $\mathrm{f} 1(\mathrm{ppm})$

S82 


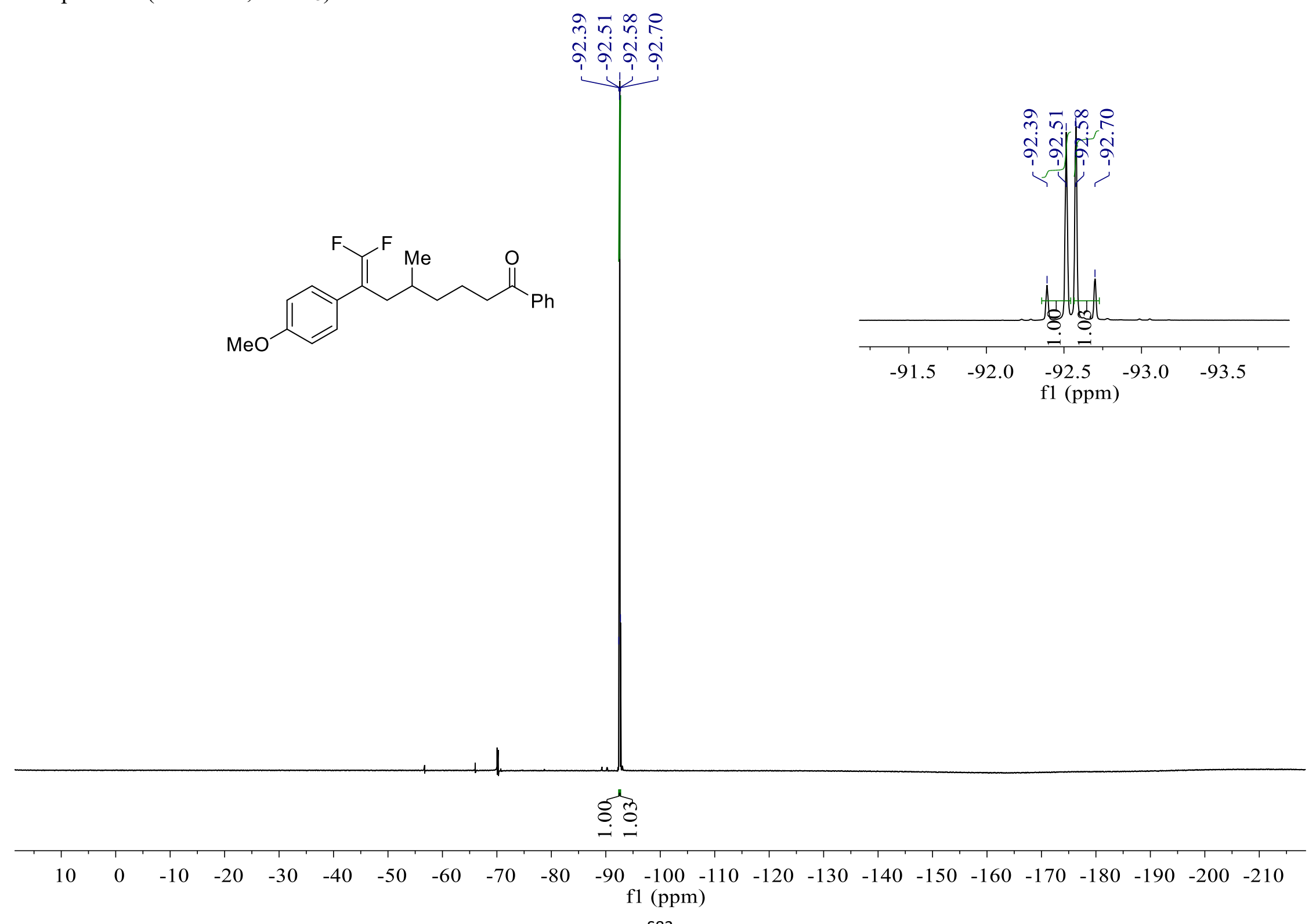

S83 


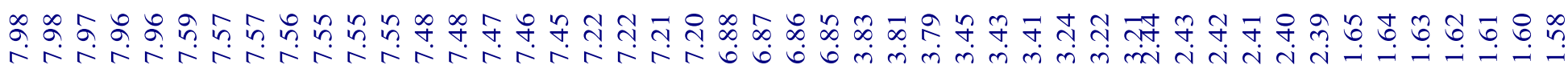

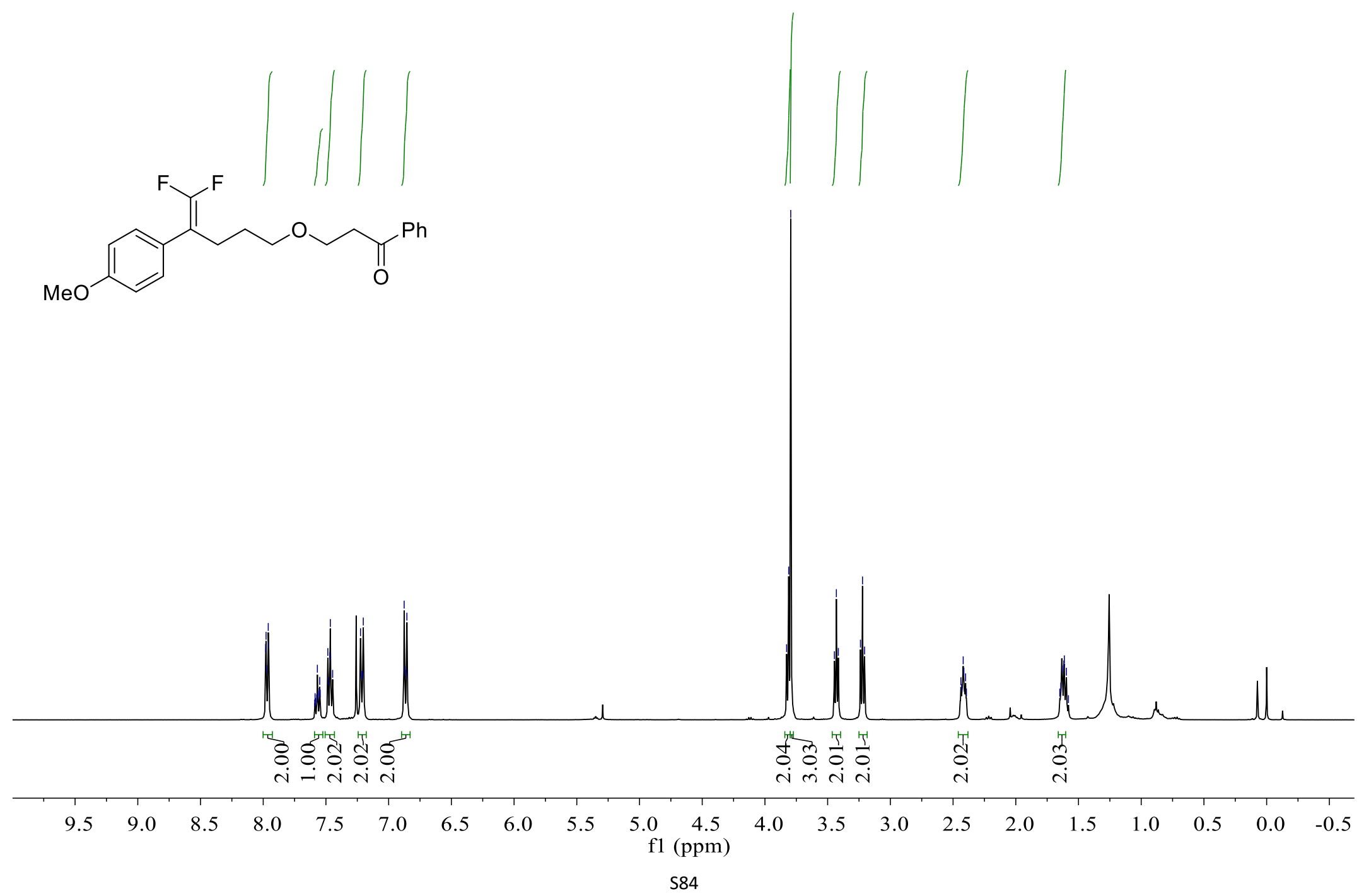


${ }^{13} \mathrm{C}\left\{{ }^{1} \mathrm{H}\right\}$ NMR-spectrum (126 MHz, $\left.\mathrm{CDCl}_{3}\right)$ of $\mathbf{3 a f}$

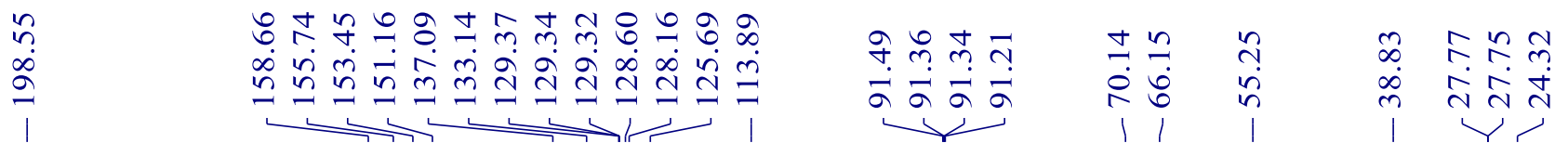

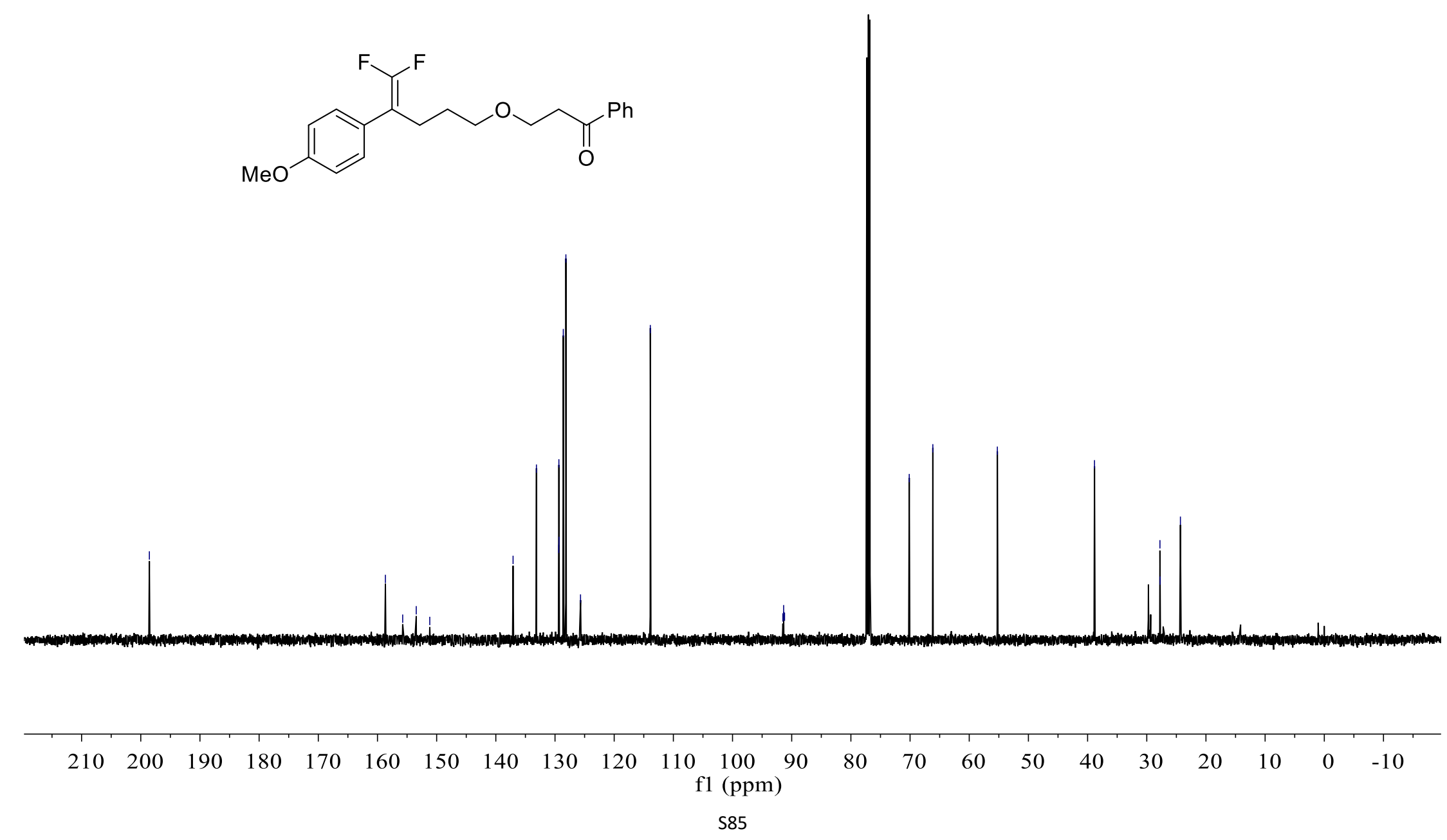


${ }^{19} \mathrm{~F}$ NMR-spectrum $\left(376 \mathrm{MHz}, \mathrm{CDCl}_{3}\right)$ of $\mathbf{3 a f}$

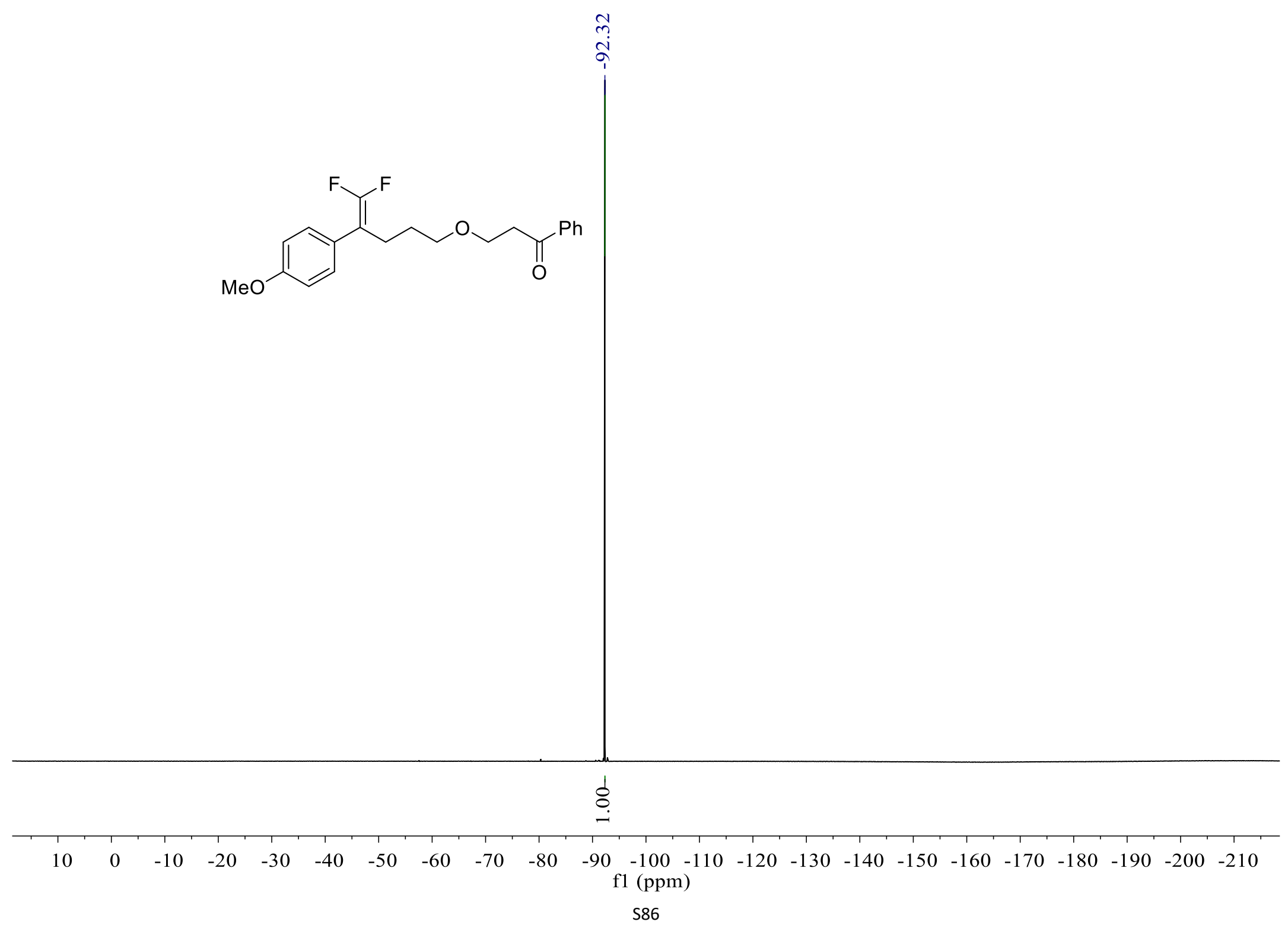




\section{${ }^{1} \mathrm{H}$ NMR-spectrum $\left(400 \mathrm{MHz}, \mathrm{CDCl}_{3}\right)$ of $\mathbf{3 a g}$}

ప

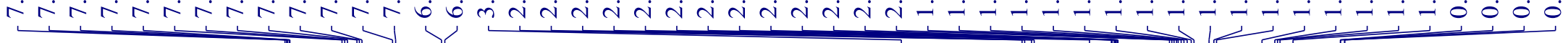

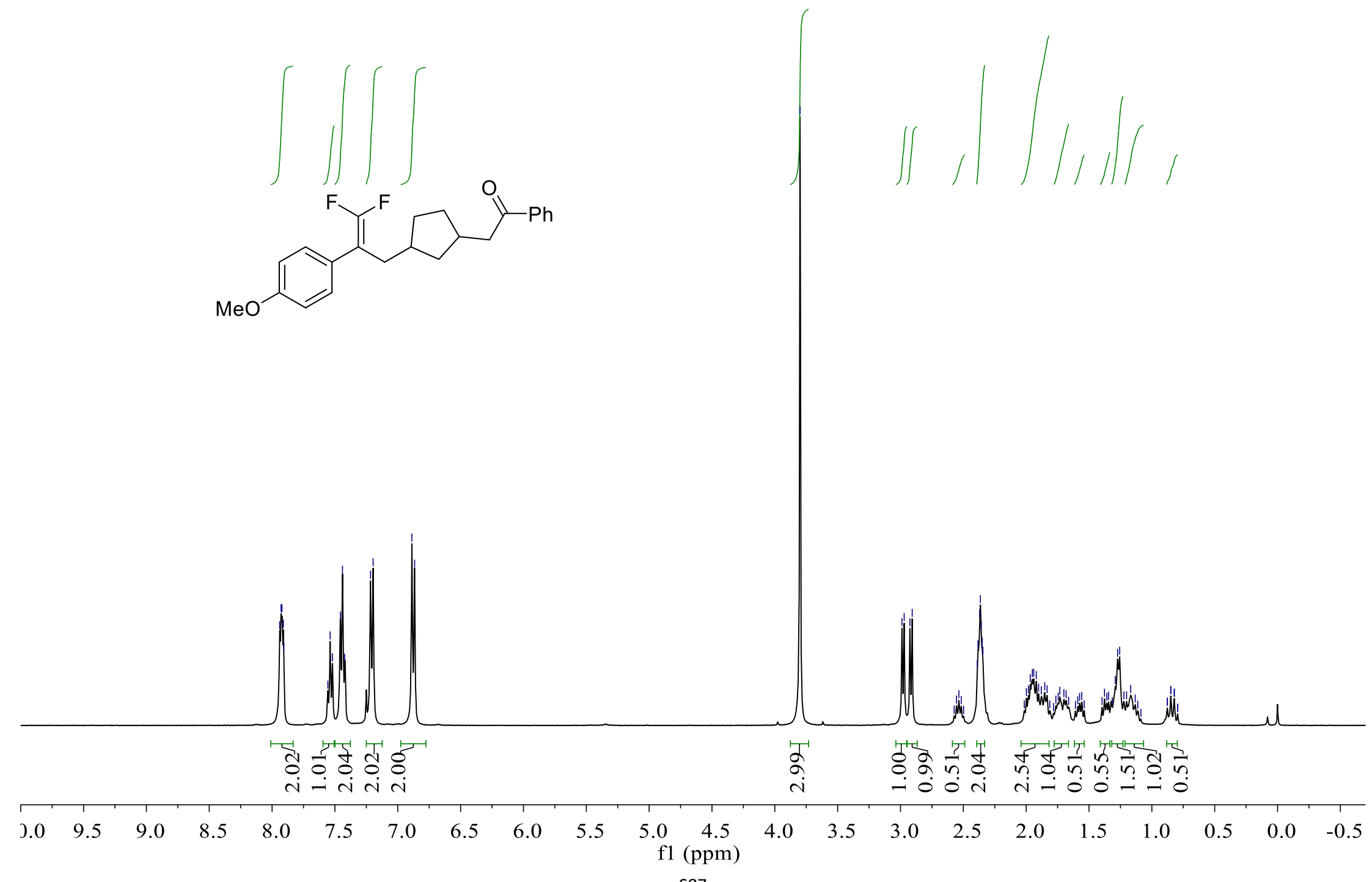


${ }^{13} \mathrm{C}\left\{{ }^{1} \mathrm{H}\right\}$ NMR-spectrum $\left(126 \mathrm{MHz}, \mathrm{CDCl}_{3}\right)$ of $\mathbf{3 a g}$
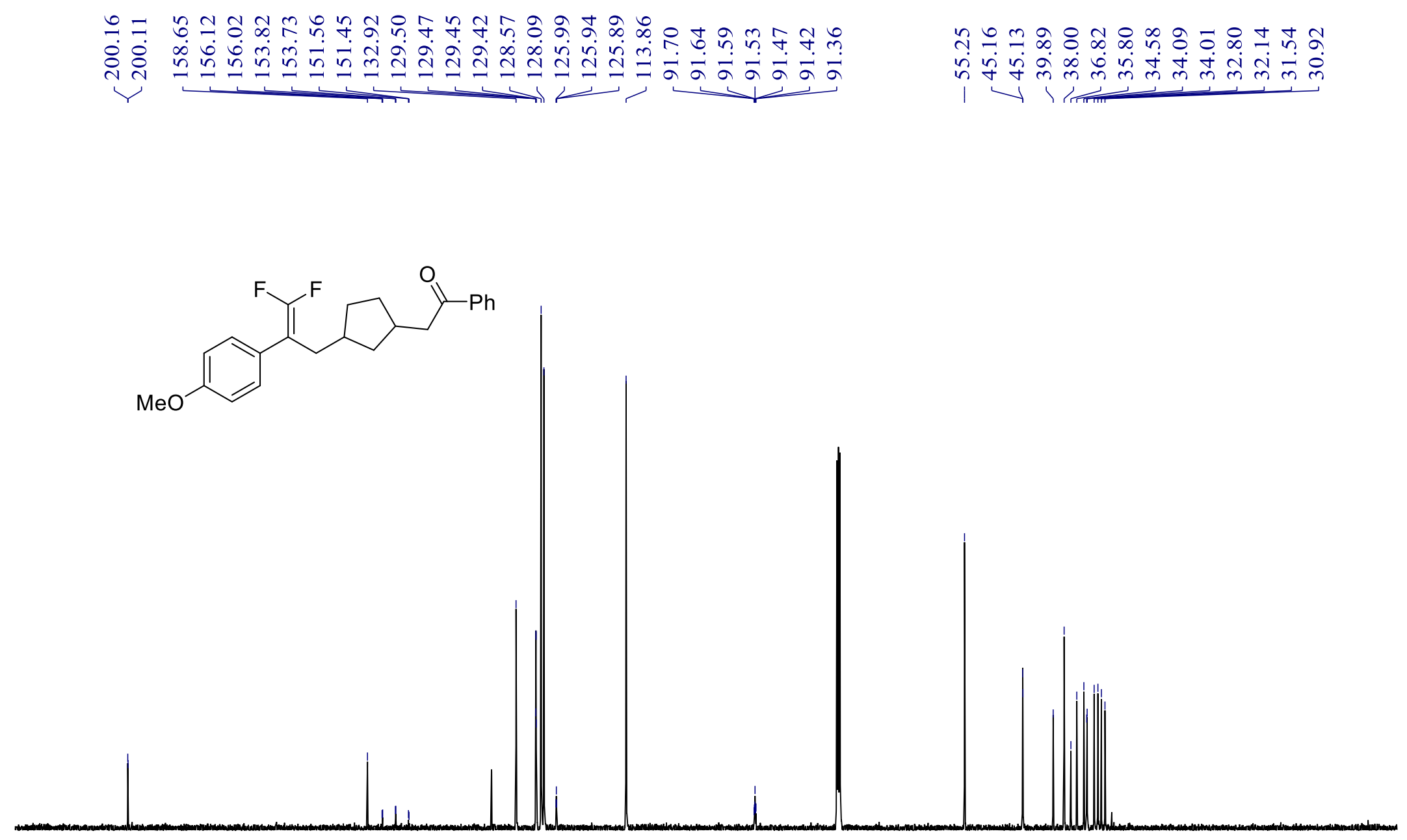

$\begin{array}{lllllllllllllllllllllll}210 & 200 & 190 & 180 & 170 & 160 & 150 & 140 & 130 & 120 & 110 & 100 & 90 & 80 & 70 & 60 & 50 & 40 & 30 & 20 & 10 & 0 & -10\end{array}$

f1 (ppm)

S88 
${ }^{19} \mathrm{~F}$ NMR-spectrum $\left(276 \mathrm{MHz}, \mathrm{CDCl}_{3}\right)$ of $\mathbf{3 a g}$

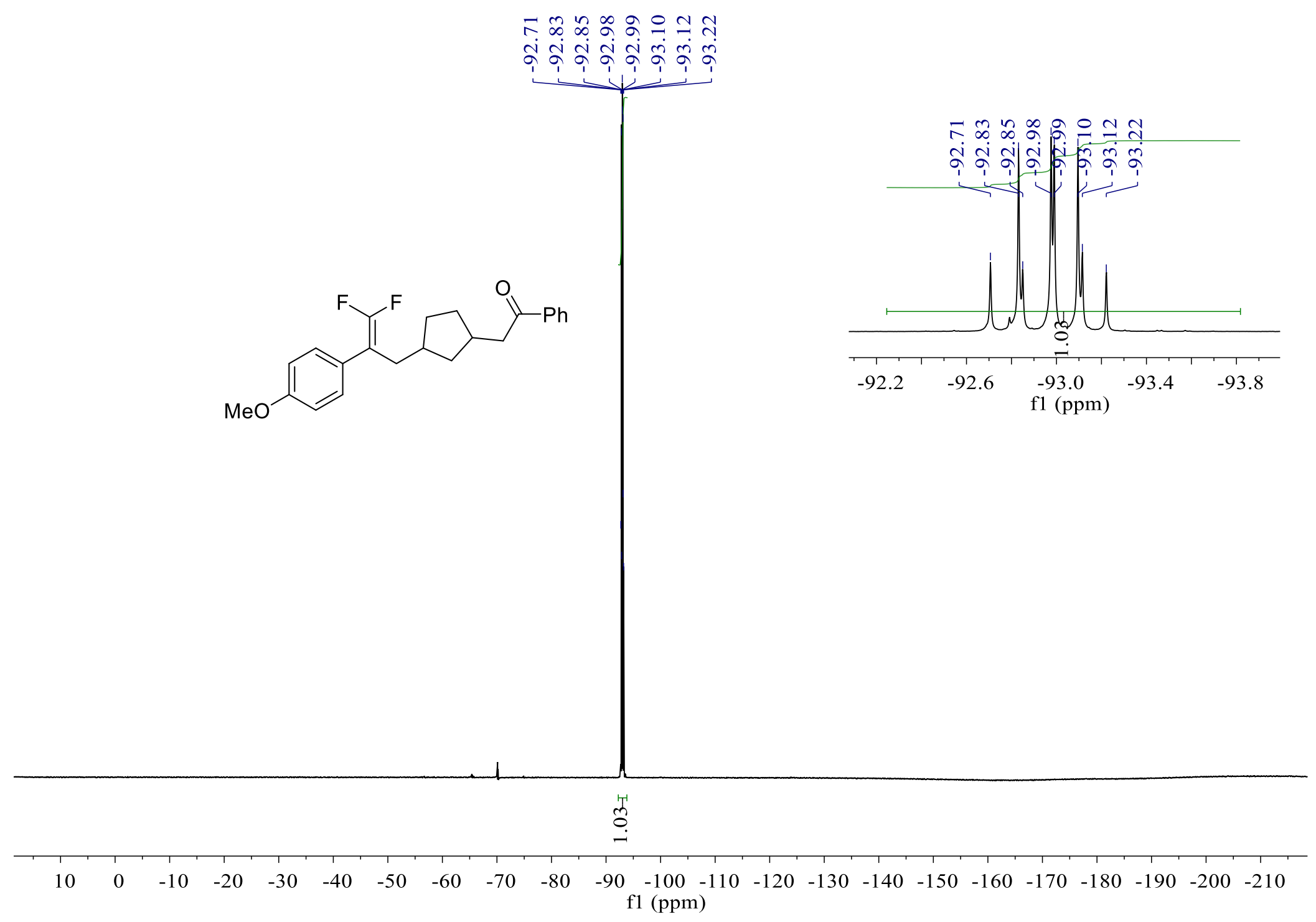


${ }^{1} \mathrm{H}$ NMR-spectrum $\left(500 \mathrm{MHz}, \mathrm{CDCl}_{3}\right)$ of $\mathbf{3 a h}$

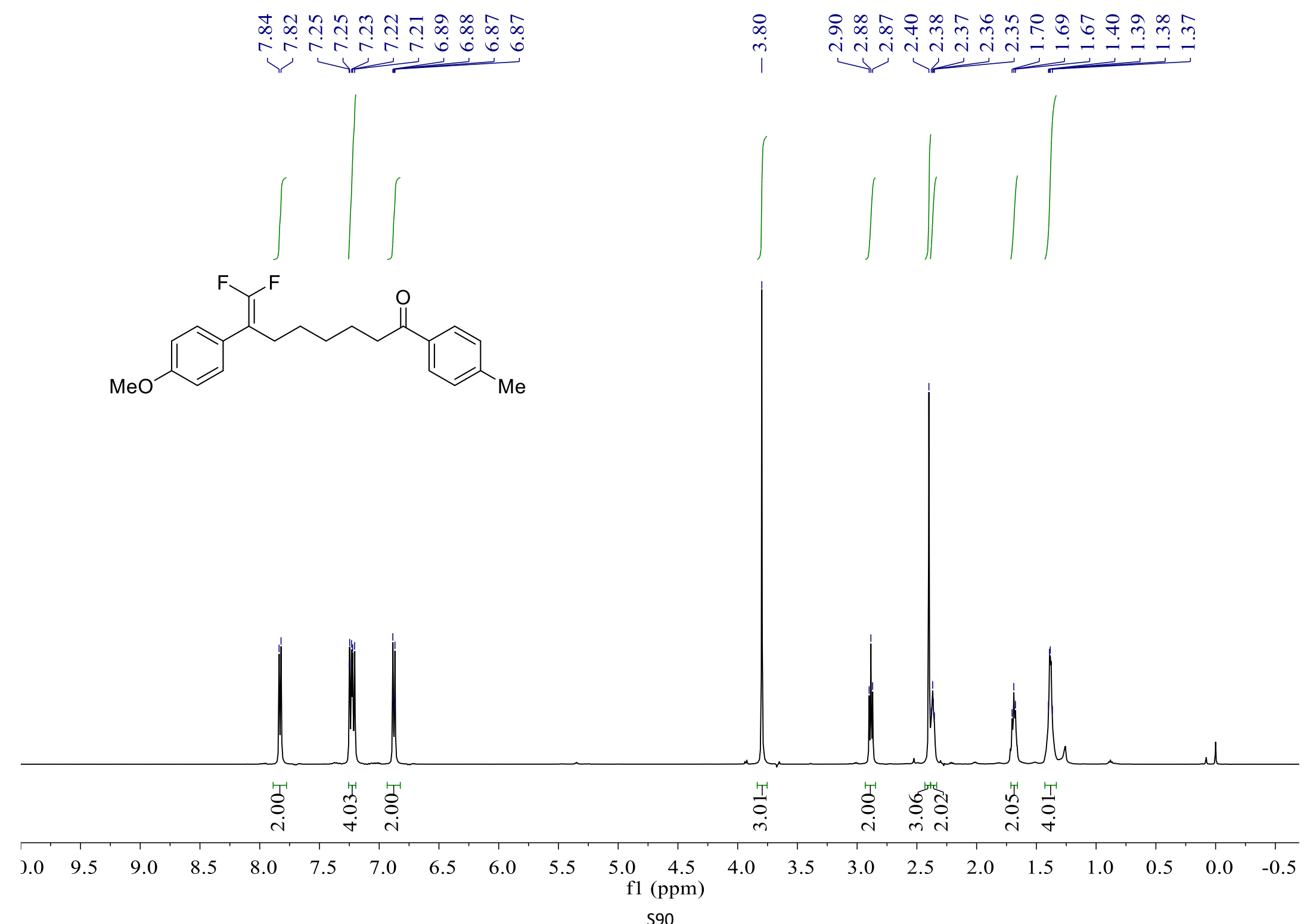


${ }^{13} \mathrm{C}\left\{{ }^{1} \mathrm{H}\right\}$ NMR-spectrum $\left(126 \mathrm{MHz}, \mathrm{CDCl}_{3}\right)$ of $\mathbf{3 a h}$

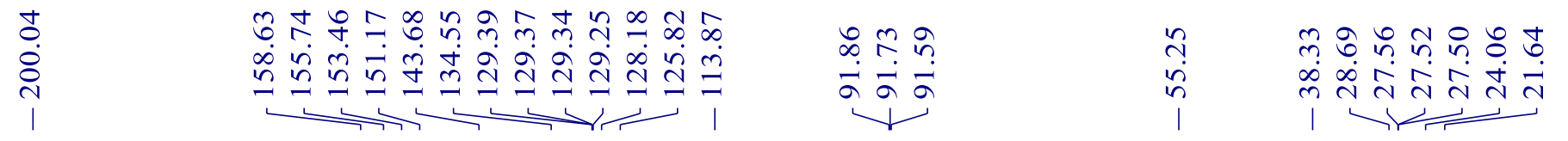

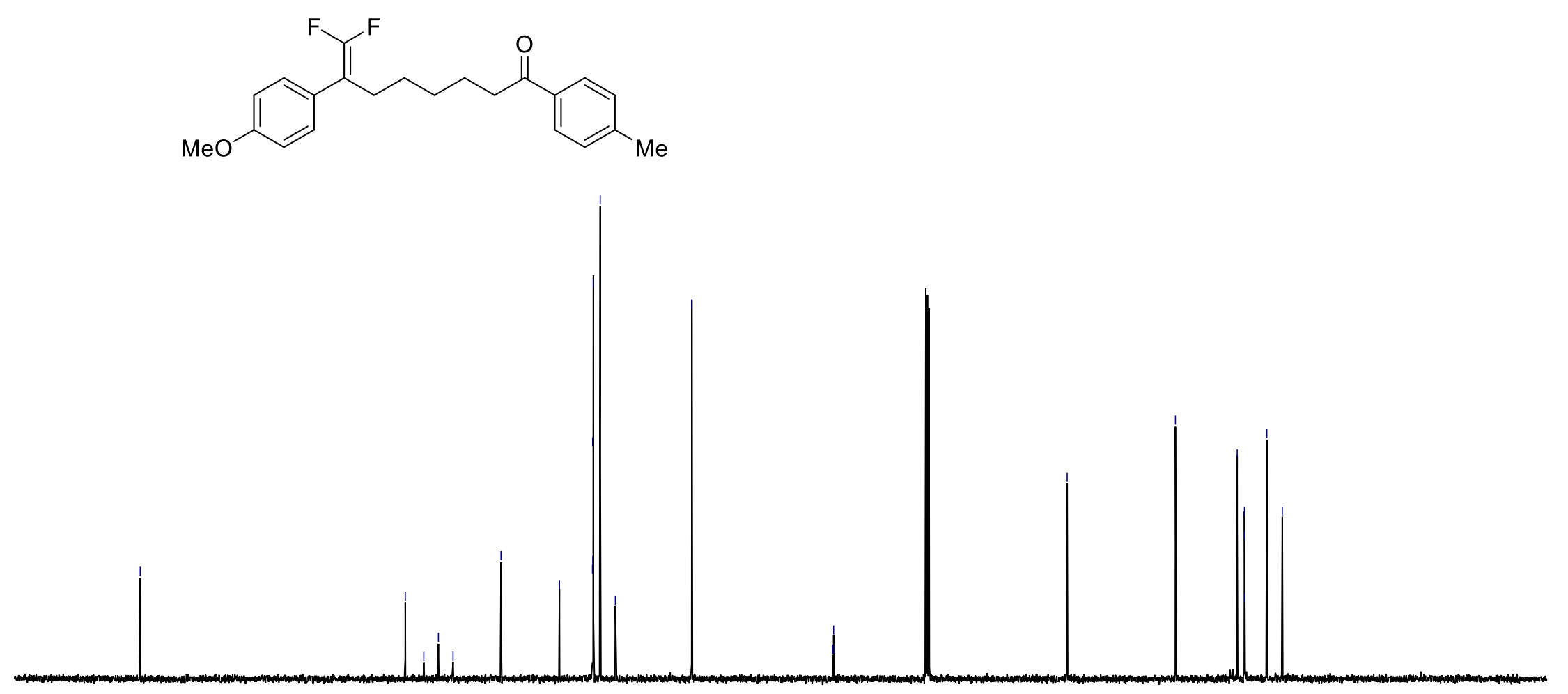

$\begin{array}{lllllllllllll}210 & 200 & 190 & 180 & 170 & 160 & 150 & 140 & 130 & 120 & 110 & 100 & 90\end{array}$

f1 (ppm)

s91 
${ }^{19} \mathrm{~F}$ NMR-spectrum (471 MHz, $\mathrm{CDCl}_{3}$ ) of $\mathbf{3 a h}$

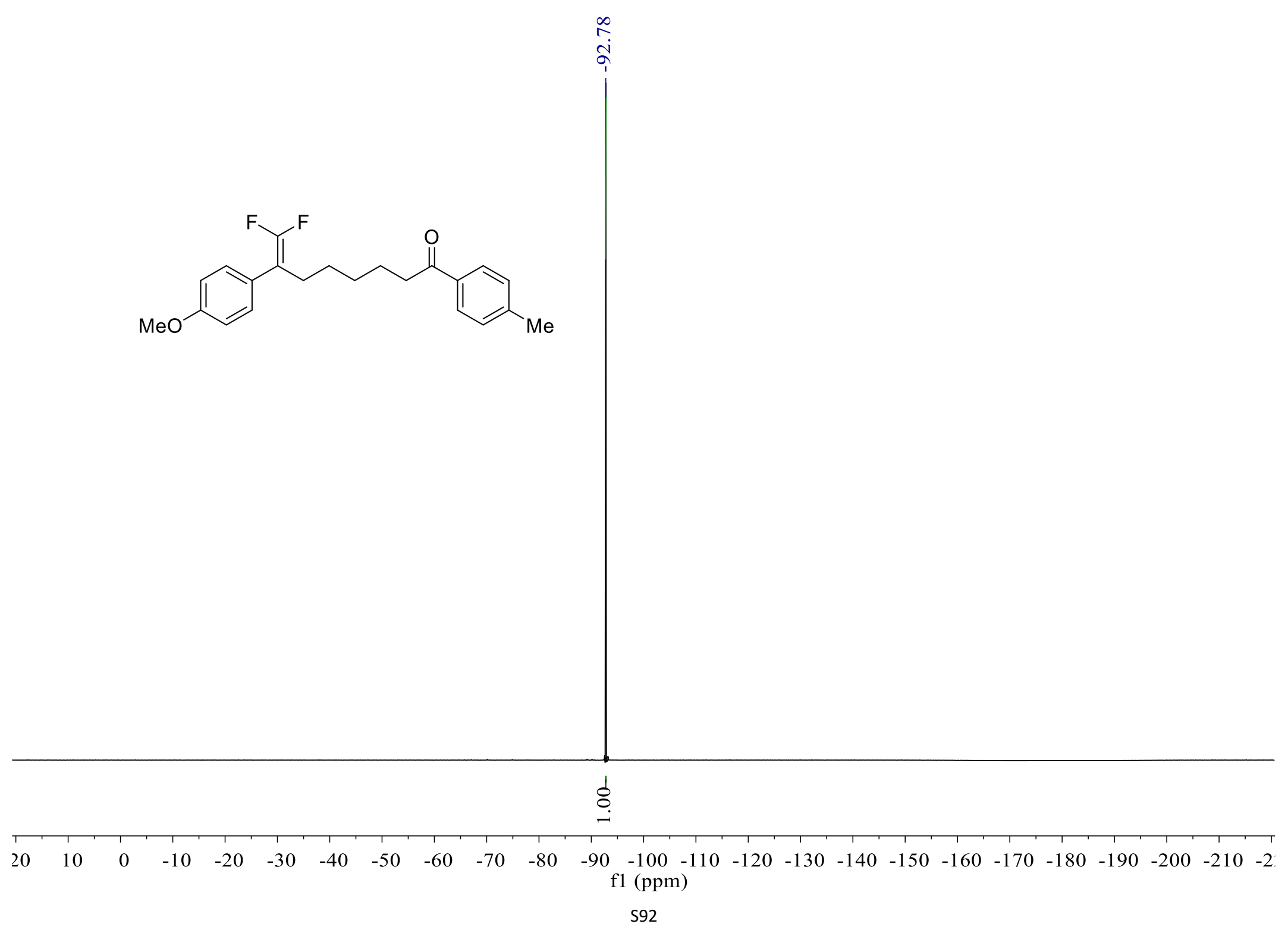


${ }^{1} \mathrm{H}$ NMR-spectrum $\left(400 \mathrm{MHz}, \mathrm{CDCl}_{3}\right)$ of $\mathbf{3 a i}$

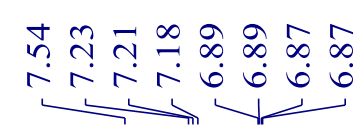

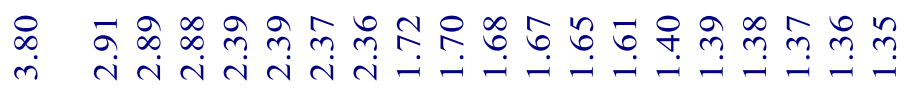

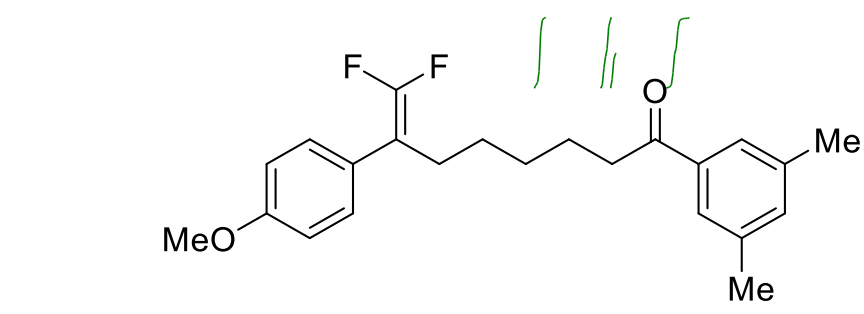

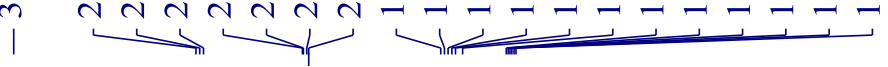

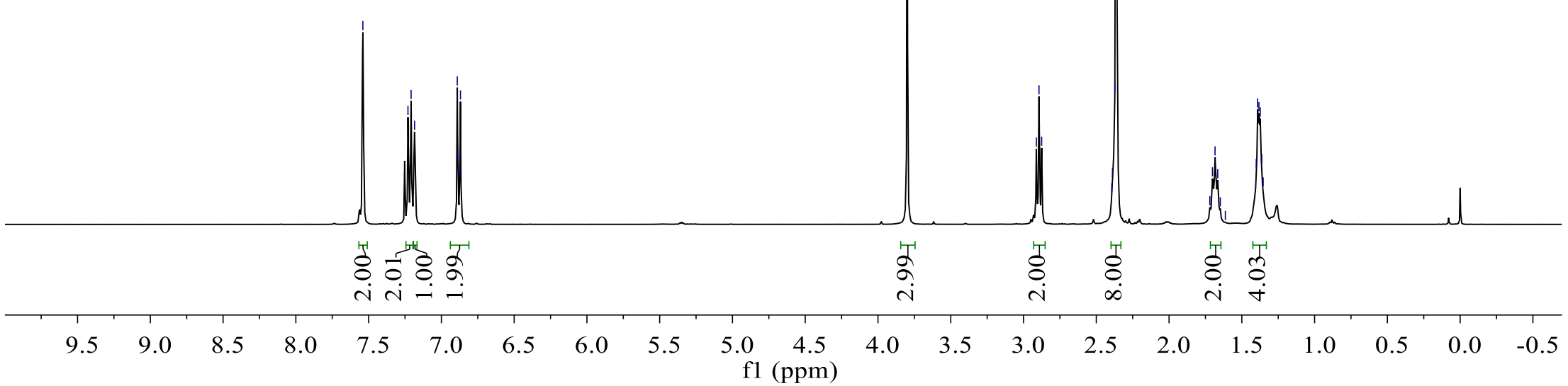

593 
${ }^{13} \mathrm{C}\left\{{ }^{1} \mathrm{H}\right\}$ NMR-spectrum $\left(400 \mathrm{MHz}, \mathrm{CDCl}_{3}\right)$ of 3ai

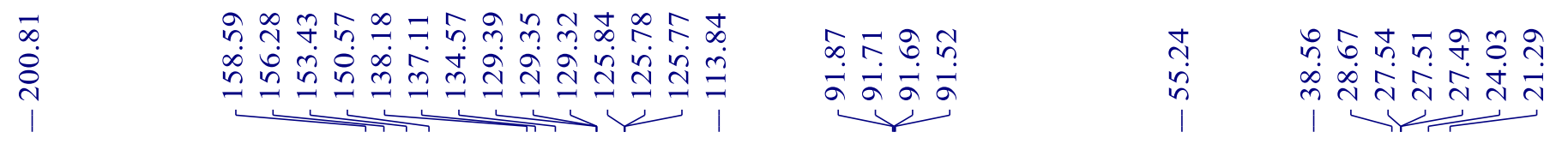<smiles>COc1ccc(C(CCCCCC(=O)c2cc(C)cc(C)c2)=C(F)F)cc1</smiles>

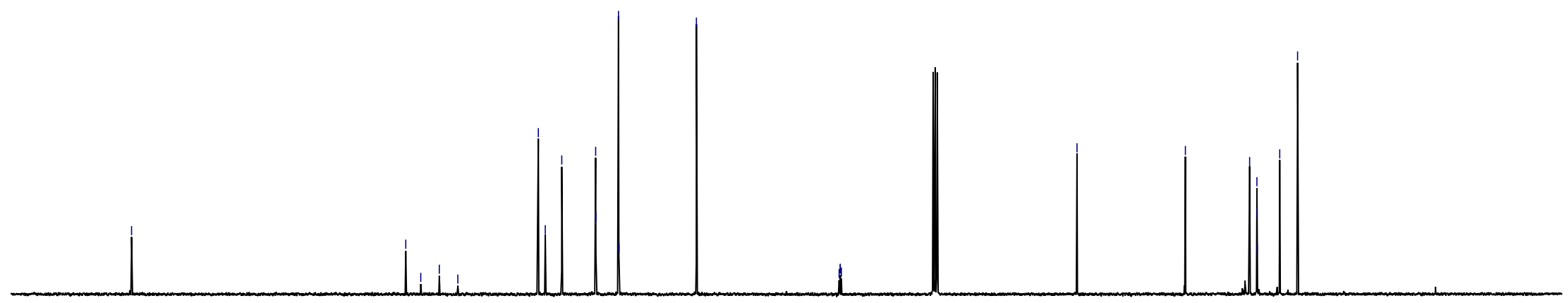

$\begin{array}{lllllllllllll}210 & 200 & 190 & 180 & 170 & 160 & 150 & 140 & 130 & 120 & 110 & 100 & 90\end{array}$

$\mathrm{f} 1(\mathrm{pp}$ 
${ }^{19} \mathrm{~F}$ NMR-spectrum $\left(376 \mathrm{MHz}, \mathrm{CDCl}_{3}\right)$ of $\mathbf{3 a i}$

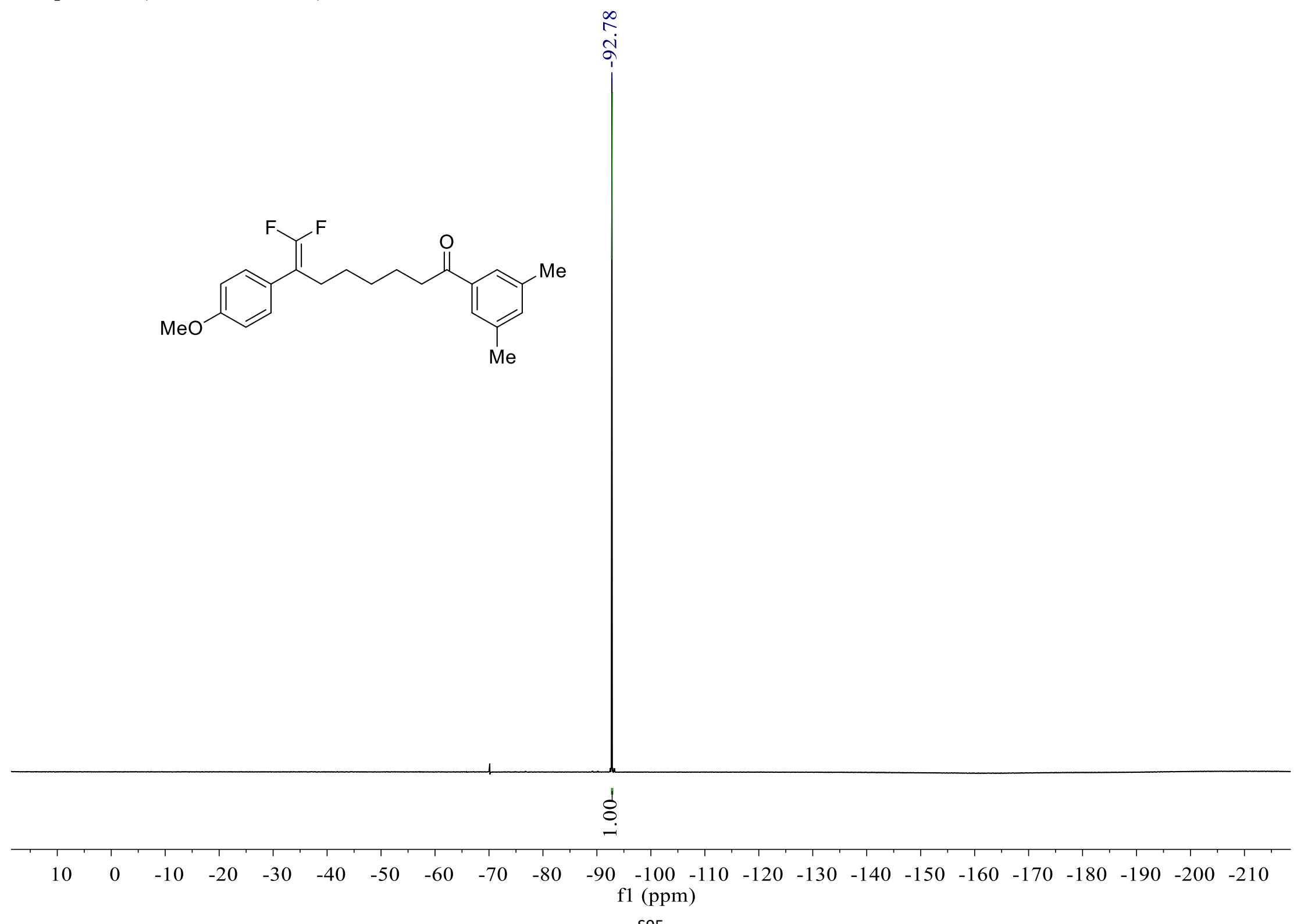

S95 


\section{${ }^{1} \mathrm{H}$ NMR-spectrum (500 MHz, $\mathrm{CDCl}_{3}$ ) of 3aj}

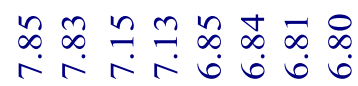

$\pi r+0.000$

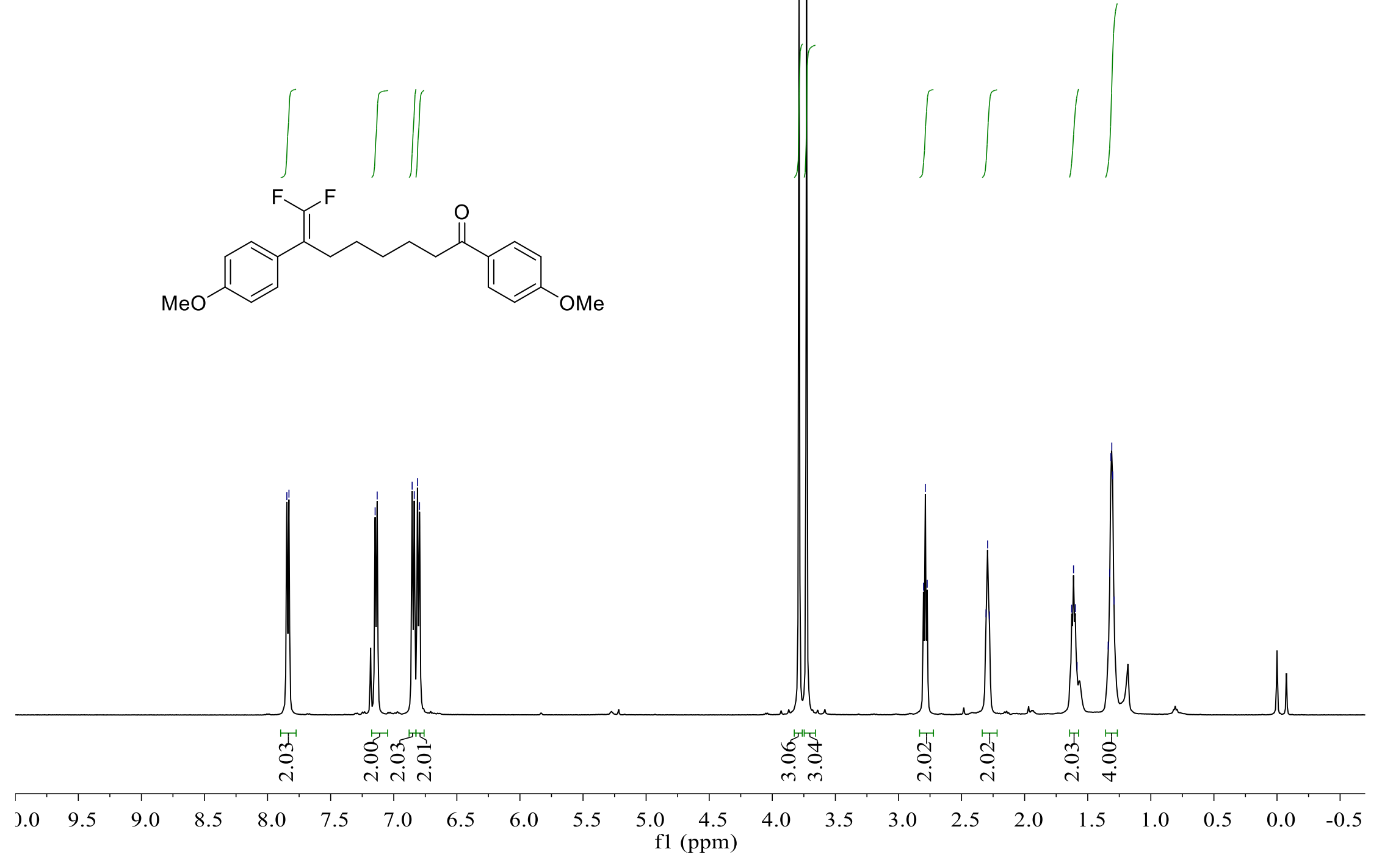

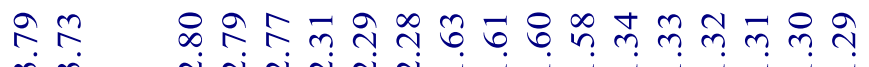

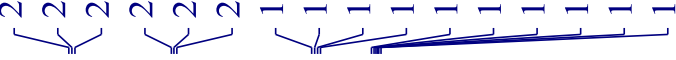

s96 
${ }^{13} \mathrm{C}\left\{{ }^{1} \mathrm{H}\right\}$ NMR-spectrum $\left(126 \mathrm{MHz}, \mathrm{CDCl}_{3}\right)$ of $\mathbf{3 a j}$

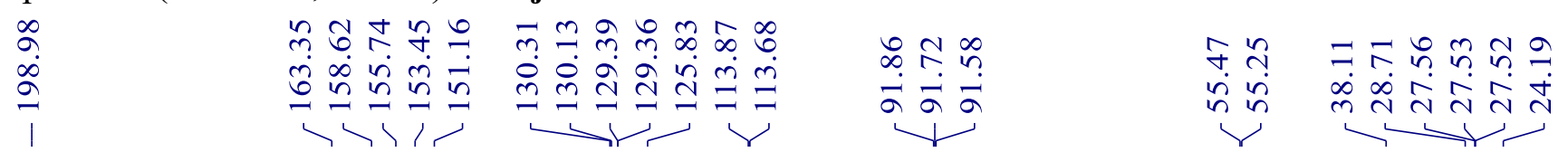<smiles>COc1ccc(C(=O)CCCCCC(=C(F)F)c2ccc(OC)cc2)cc1</smiles>

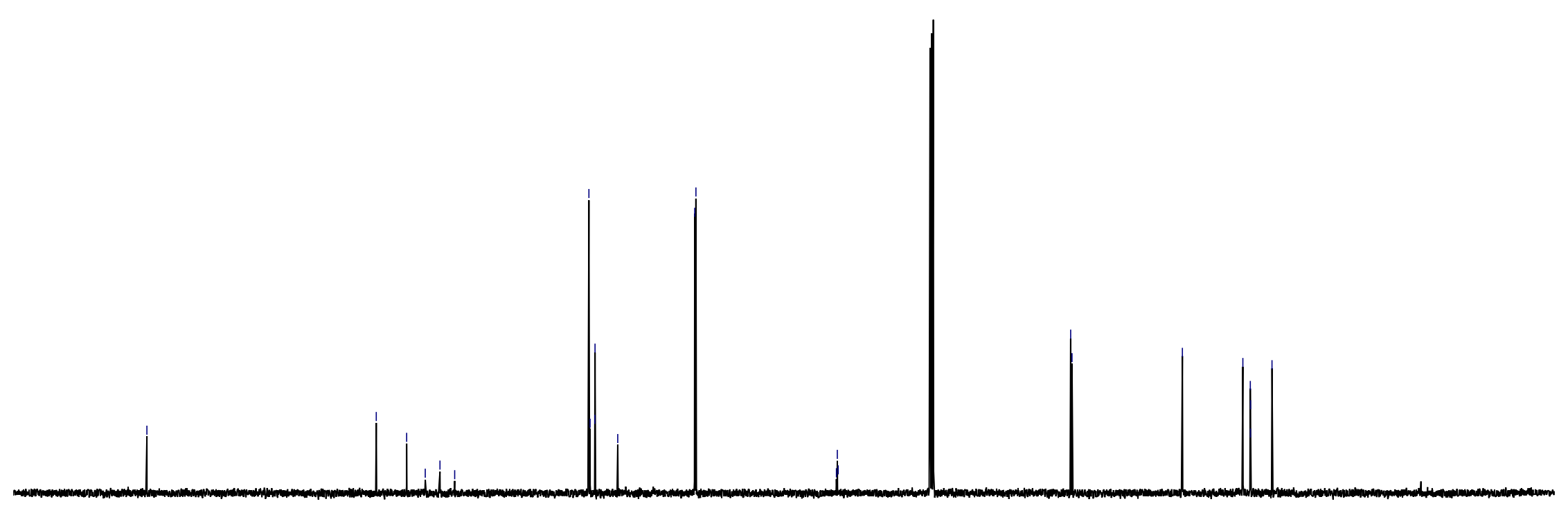

$\begin{array}{cccccccccccccccccccccccc}210 & 200 & 190 & 180 & 170 & 160 & 150 & 140 & 130 & 120 & 110 \underset{\mathrm{f} 1(\mathrm{ppm})}{100} & 90 & 80 & 70 & 60 & 50 & 40 & 30 & 20 & 10 & 0 & -10\end{array}$ s97 
${ }^{19} \mathrm{~F}$ NMR-spectrum $\left(376 \mathrm{MHz}, \mathrm{CDCl}_{3}\right)$ of $\mathbf{3 a j}$

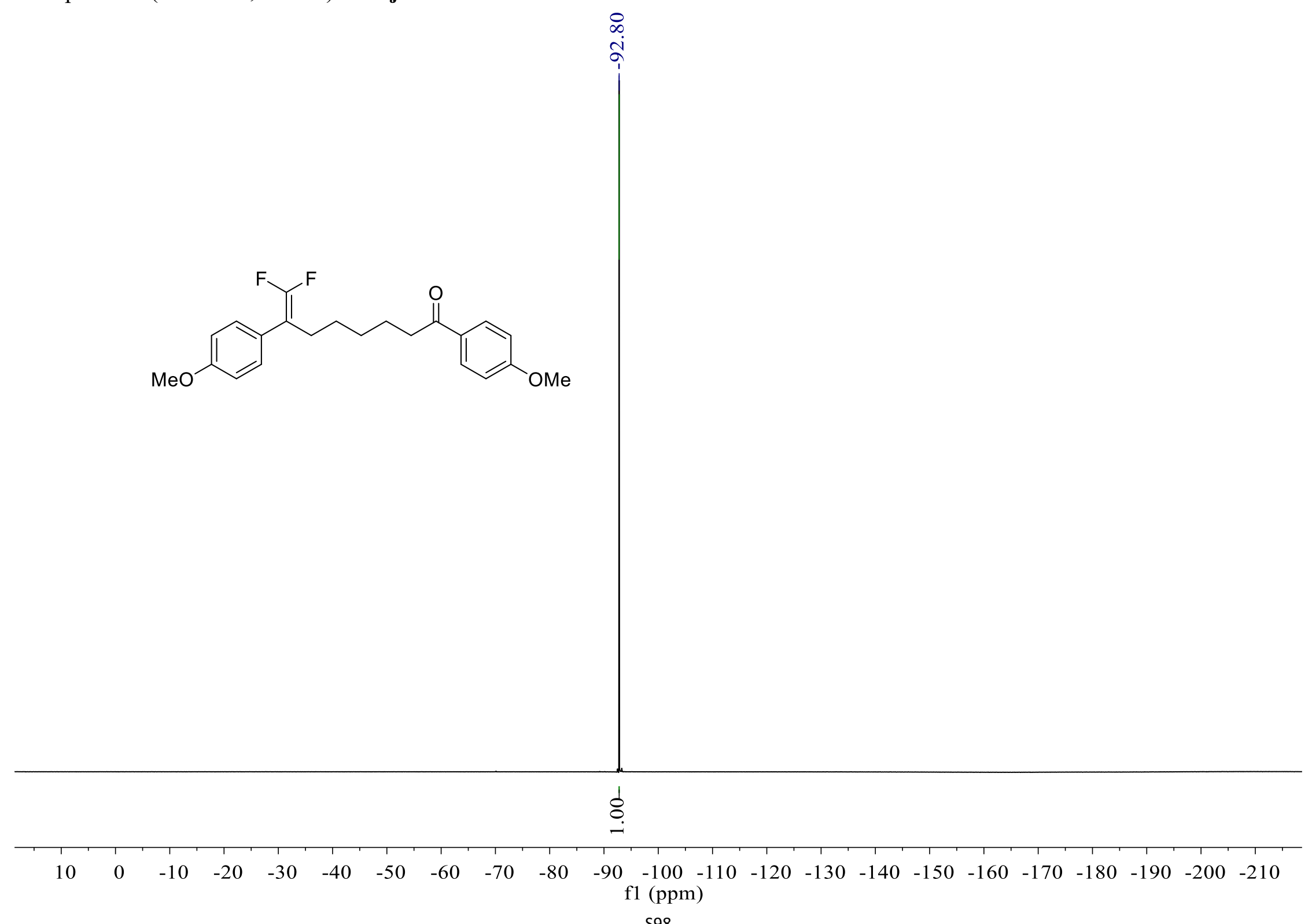

s98 
${ }^{1} \mathrm{H}$ NMR-spectrum (400 MHz, $\mathrm{CDCl}_{3}$ ) of $\mathbf{3 a k}$

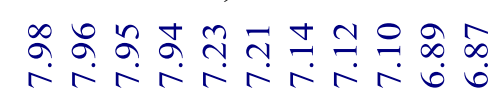

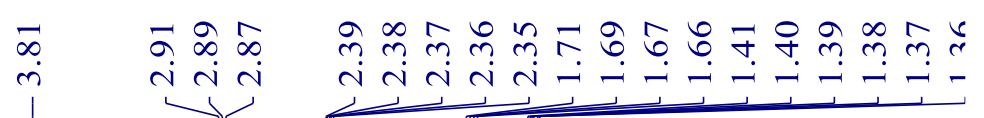

$\sqrt{5}+5 \pi 500$
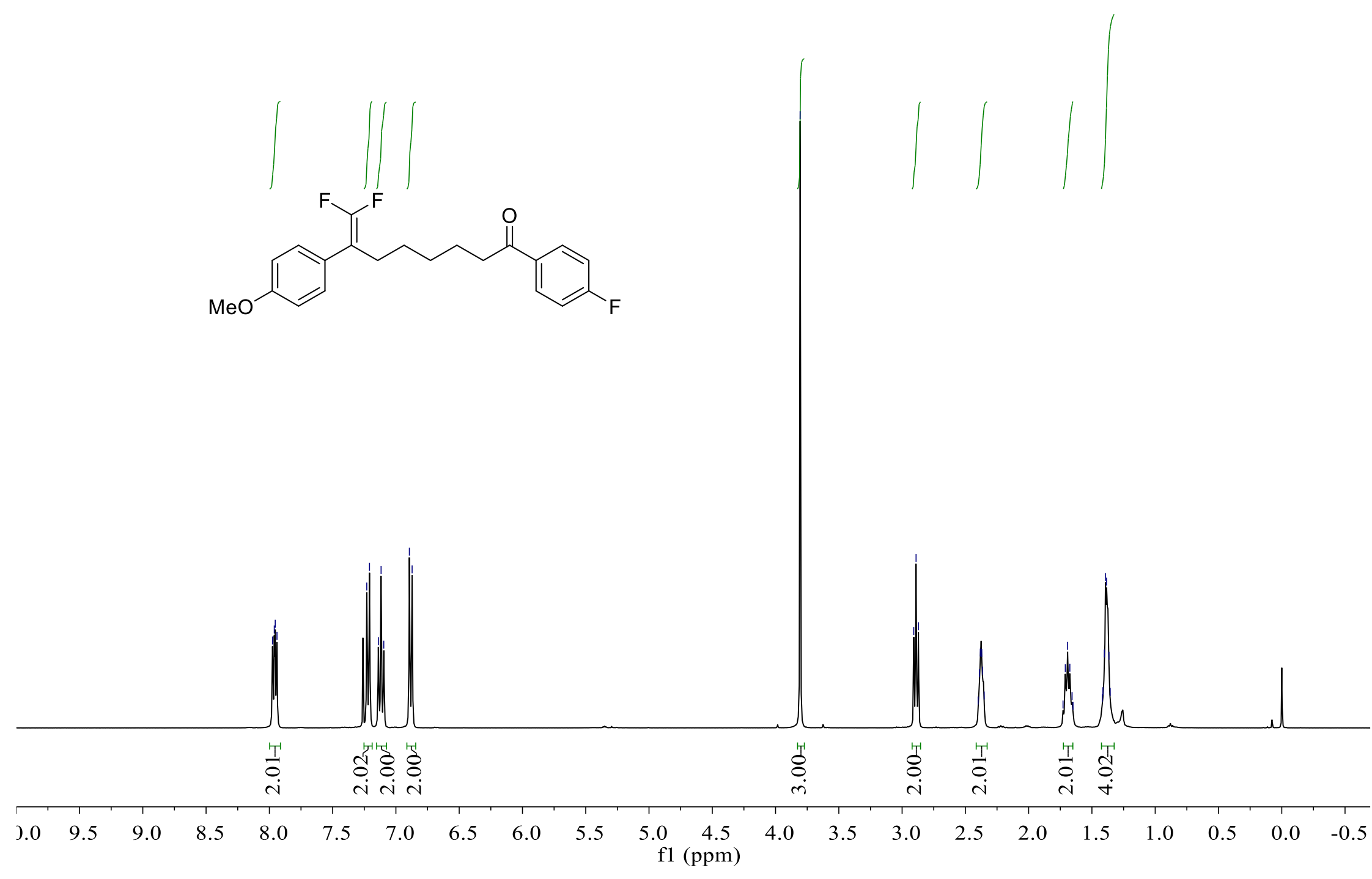

s99 
${ }^{13} \mathrm{C}\left\{{ }^{1} \mathrm{H}\right\}$ NMR-spectrum (101 MHz, $\left.\mathrm{CDCl}_{3}\right)$ of $\mathbf{3 a k}$

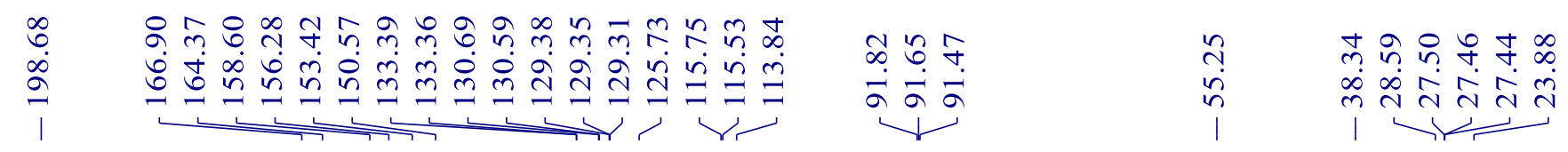

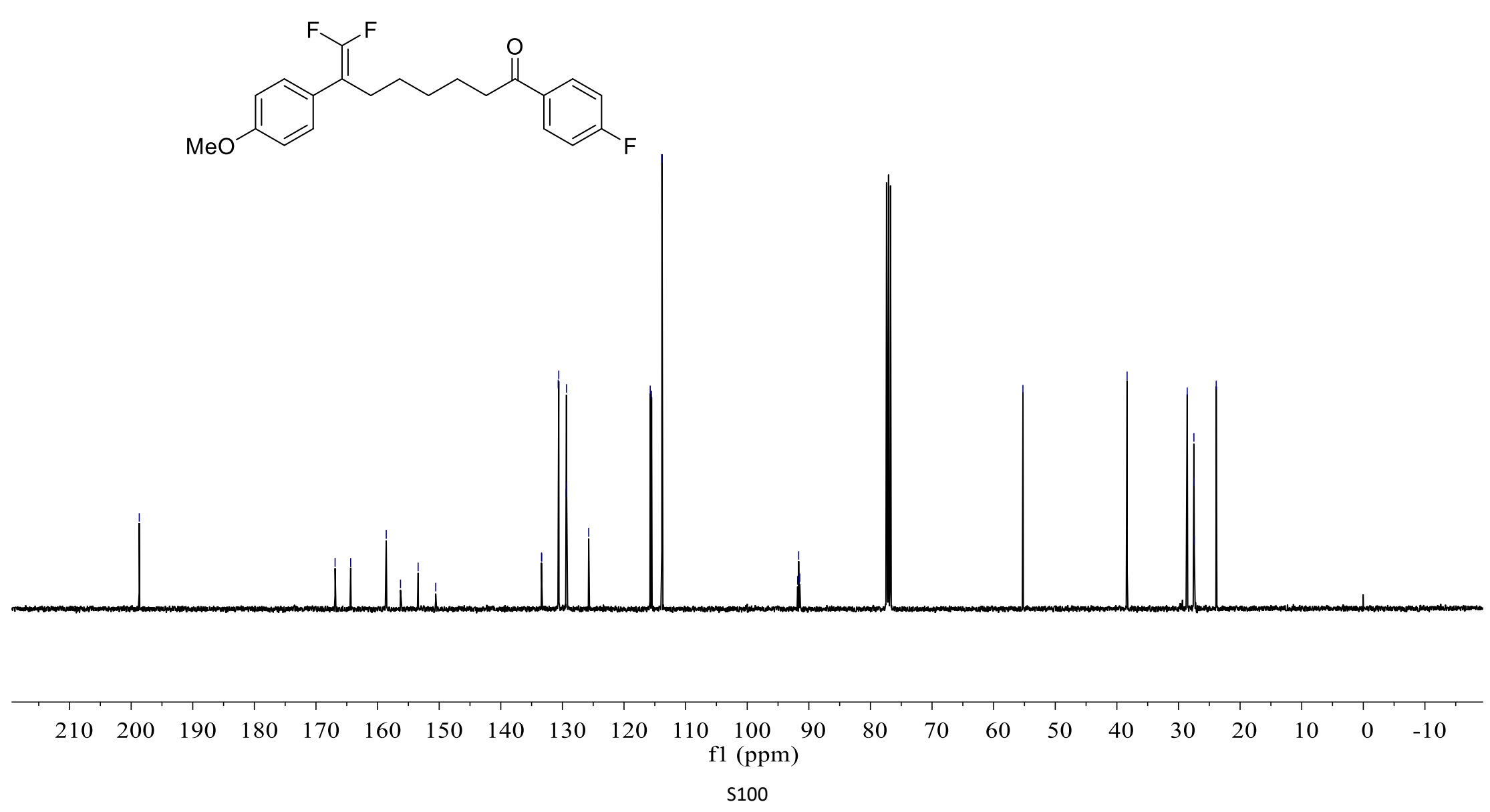


${ }^{19}$ F NMR-spectrum (376 MHz, $\mathrm{CDCl}_{3}$ ) of $\mathbf{3 a k}$

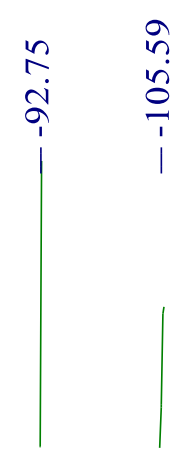

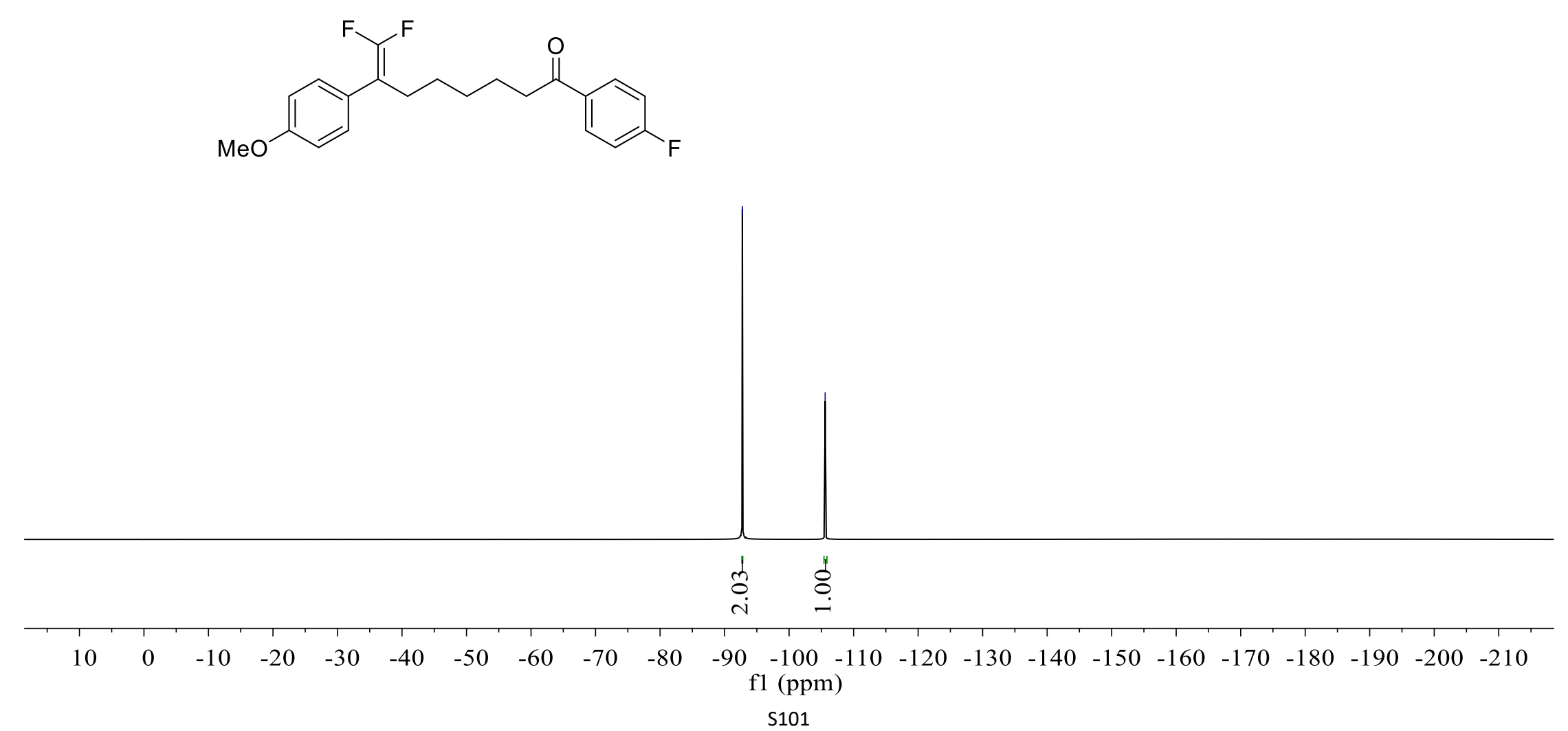




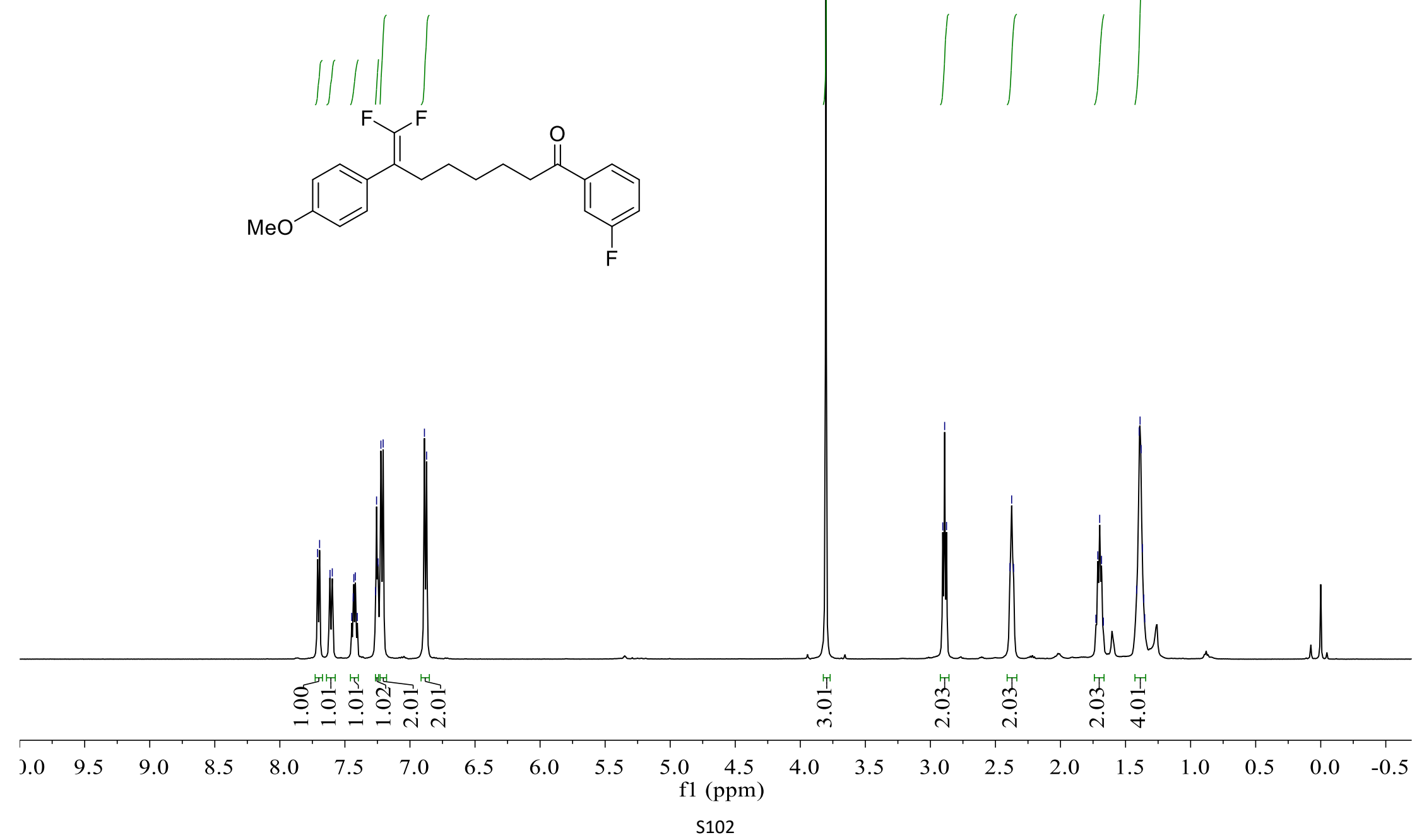


${ }^{13} \mathrm{C}\left\{{ }^{1} \mathrm{H}\right\}$ NMR-spectrum $\left(126 \mathrm{MHz}, \mathrm{CDCl}_{3}\right)$ of $\mathbf{3 a l}$

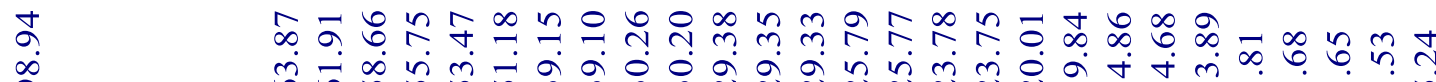

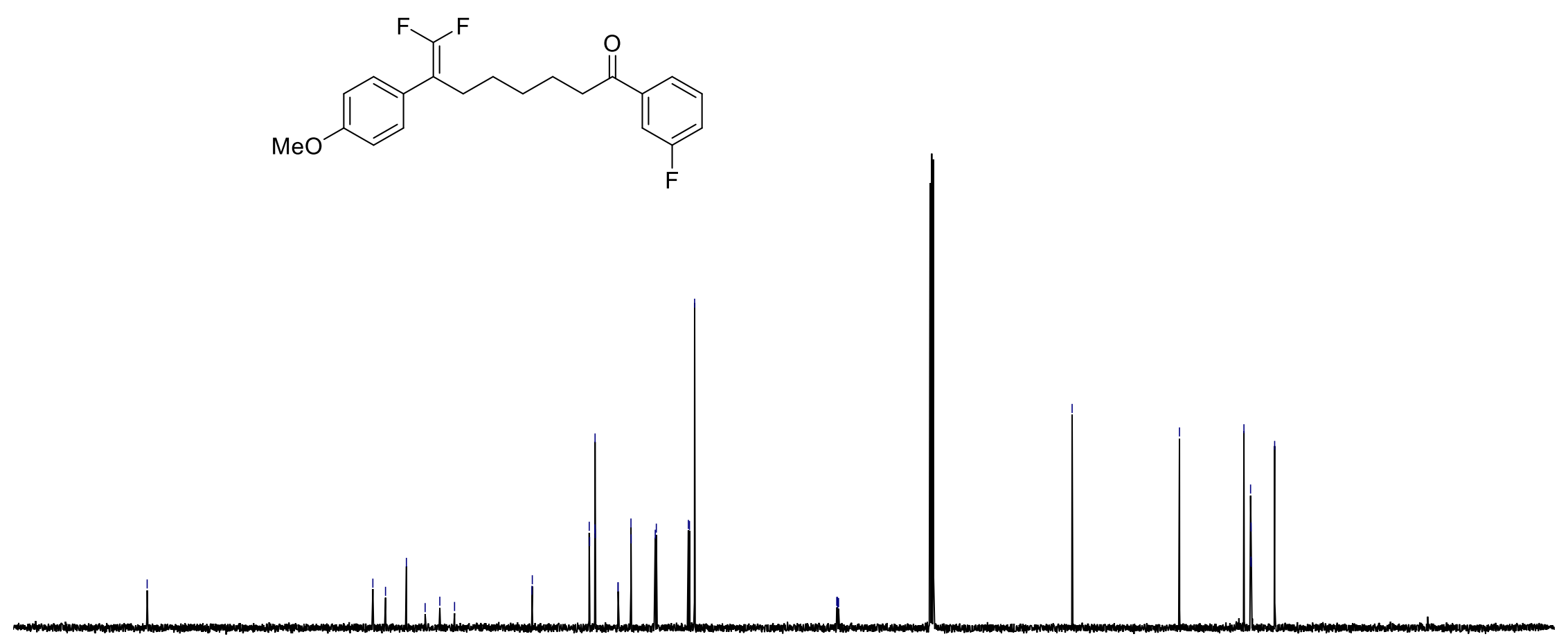

$\begin{array}{llllllllllll}210 & 200 & 190 & 180 & 170 & 160 & 150 & 140 & 130 & 120 & 110 & 100\end{array}$ f1 (ppm)

S103 
${ }^{19} \mathrm{~F}$ NMR-spectrum $\left(471 \mathrm{MHz}, \mathrm{CDCl}_{3}\right)$ of $\mathbf{3 a l}$

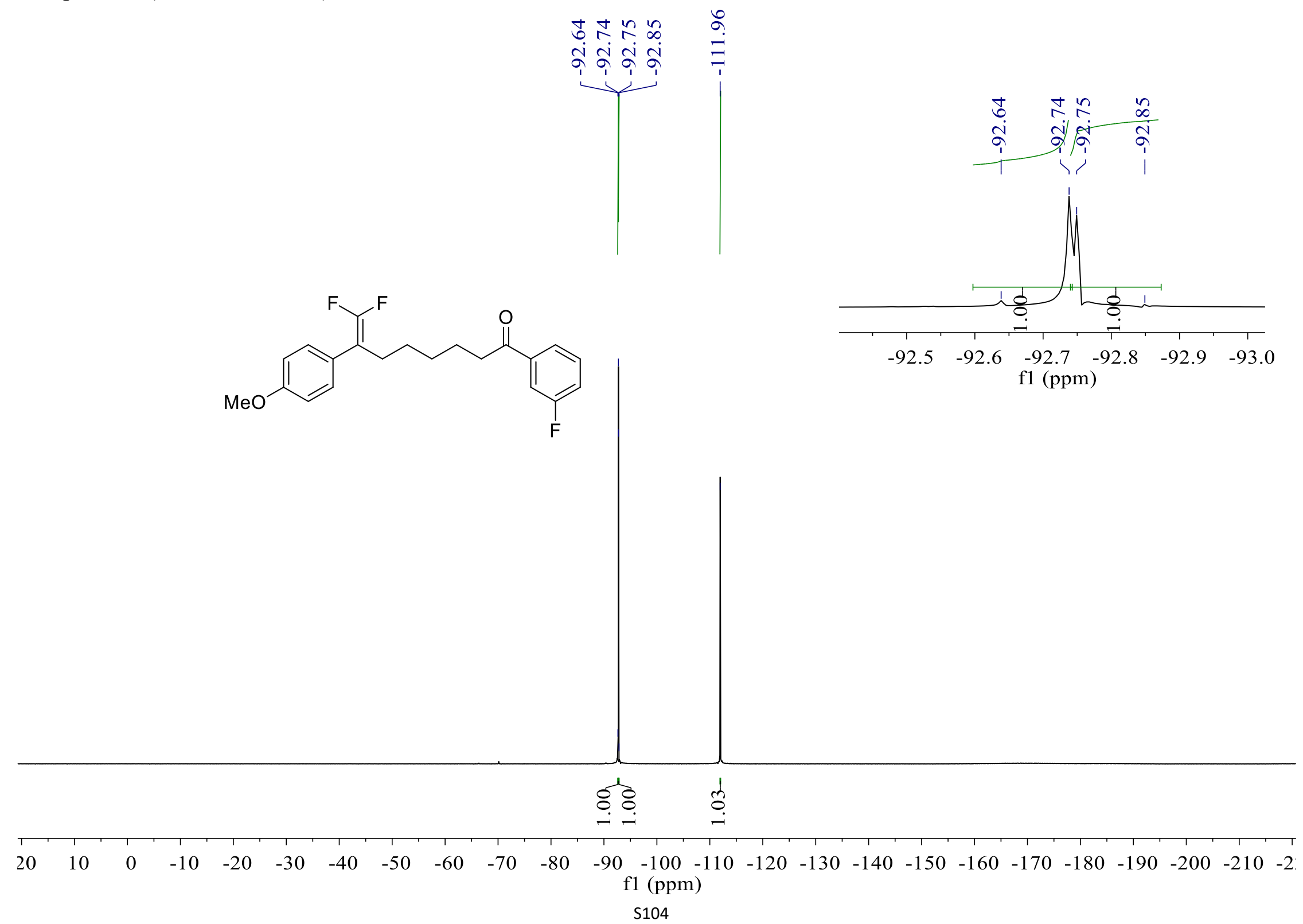




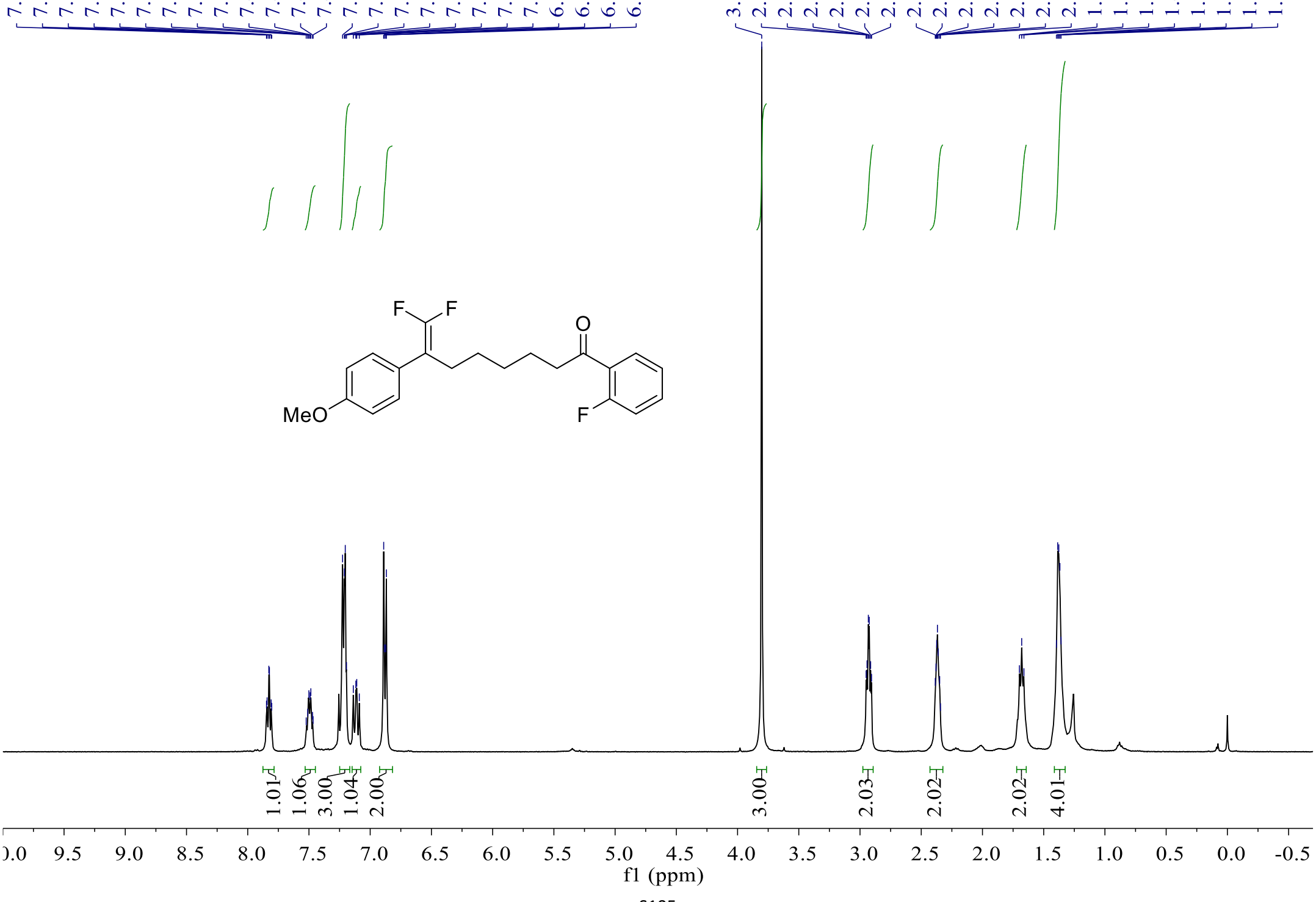


${ }^{13} \mathrm{C}\left\{{ }^{1} \mathrm{H}\right\}$ NMR-spectrum $\left(126 \mathrm{MHz}, \mathrm{CDCl}_{3}\right)$ of $\mathbf{3 a m}$
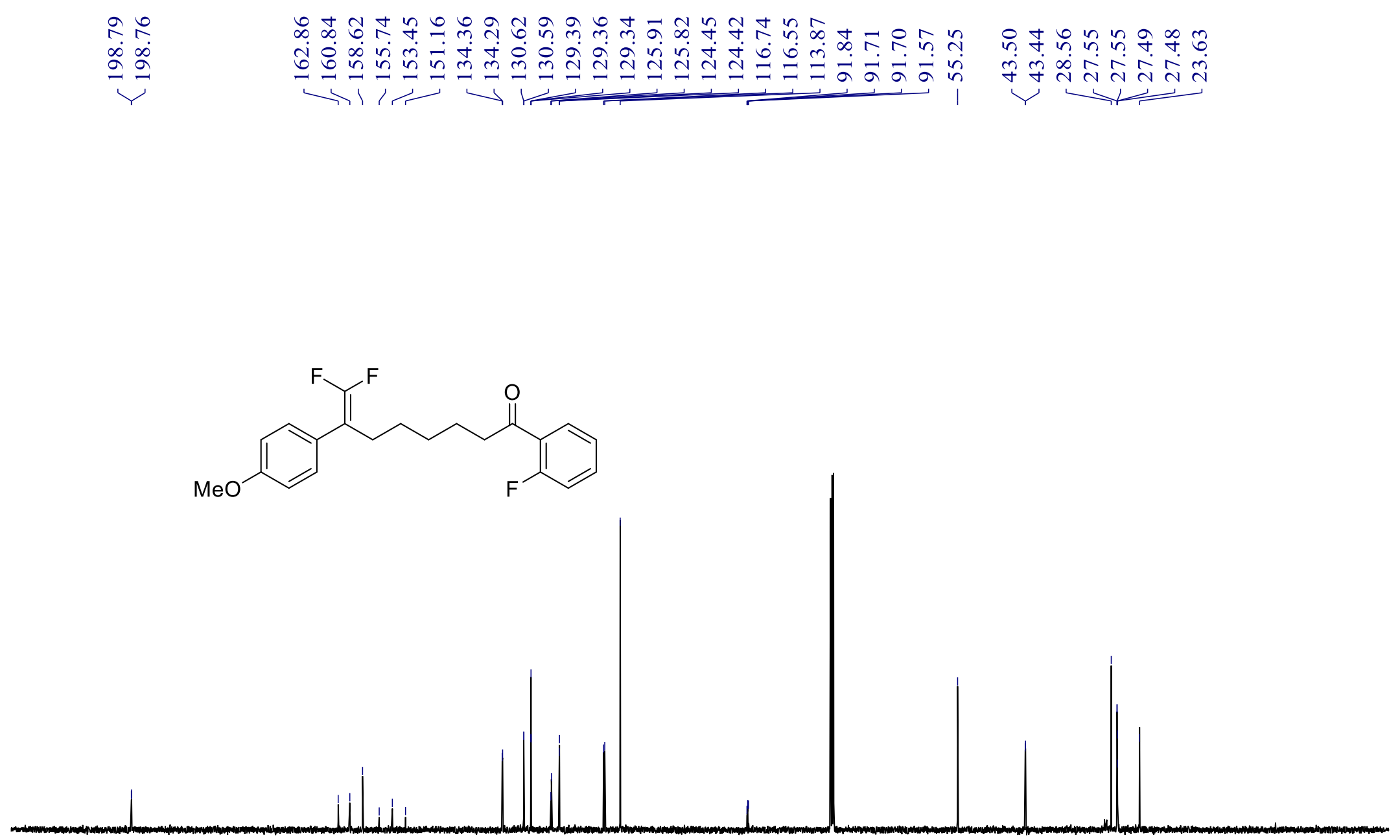

$\begin{array}{llllllllllllllllllllll}210 & 200 & 190 & 180 & 170 & 160 & 150 & 140 & 130 & 120 & 110 \begin{array}{c}100 \\ \mathrm{f} 1(\mathrm{ppm})\end{array} & 90 & 80 & 70 & 60 & 50 & 40 & 30 & 20 & 10 & 0 & -10\end{array}$ S106 
${ }^{19} \mathrm{~F}$ NMR-spectrum $\left(376 \mathrm{MHz}, \mathrm{CDCl}_{3}\right)$ of $\mathbf{3 a m}$

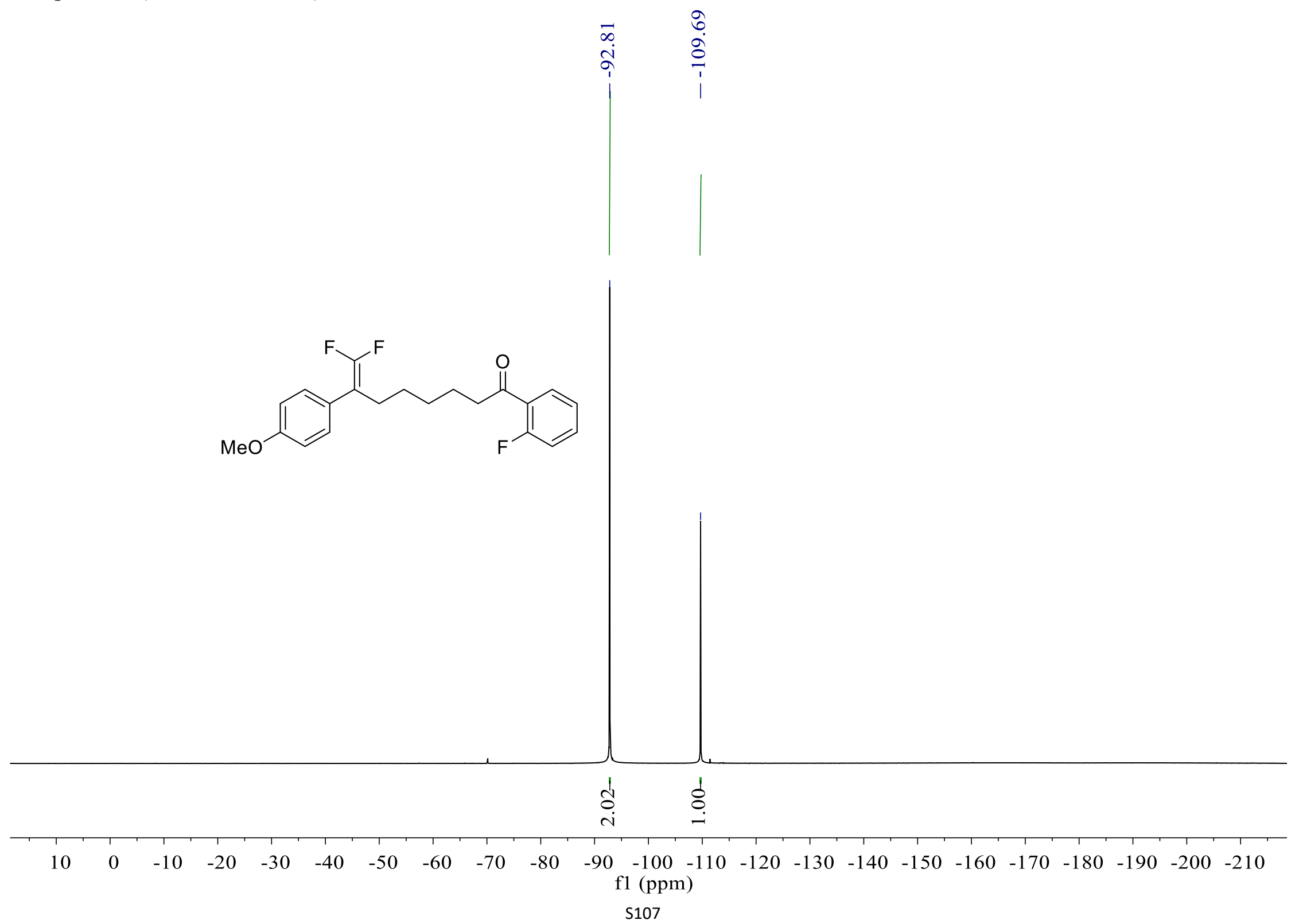




\section{${ }^{1} \mathrm{H}$ NMR-spectrum (400 MHz, $\mathrm{CDCl}_{3}$ ) of 3an}

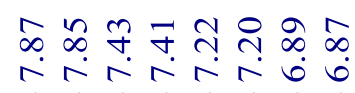

iriario

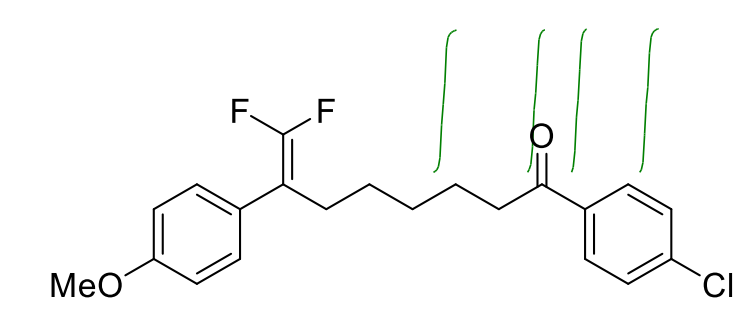

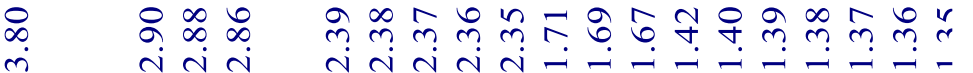

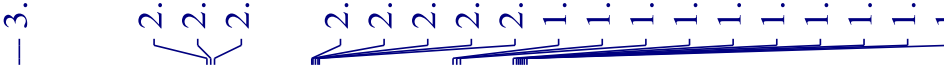

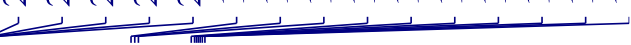

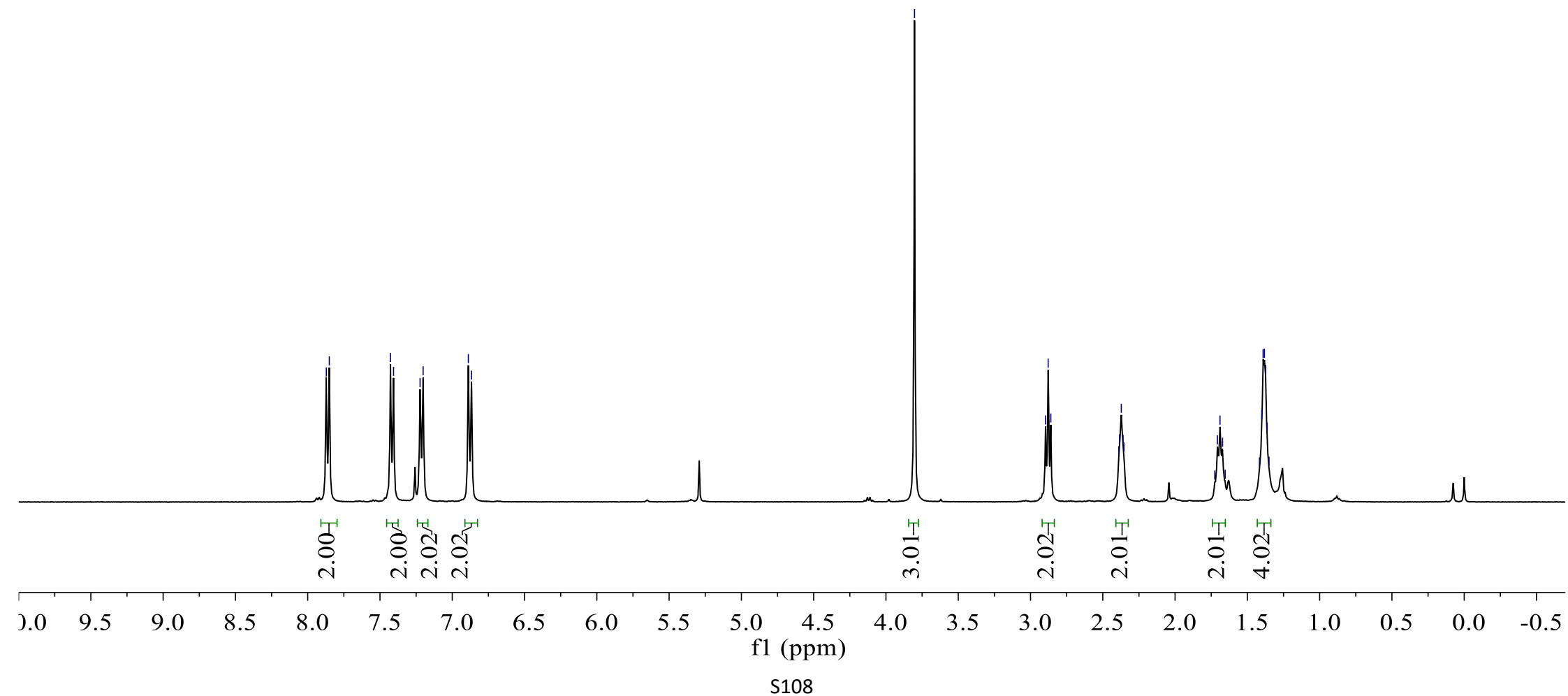


${ }^{13} \mathrm{C}\left\{{ }^{1} \mathrm{H}\right\}$ NMR-spectrum $\left(126 \mathrm{MHz}, \mathrm{CDCl}_{3}\right)$ of $\mathbf{3 a n}$

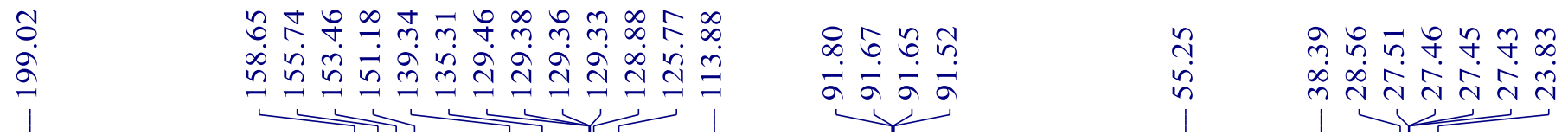

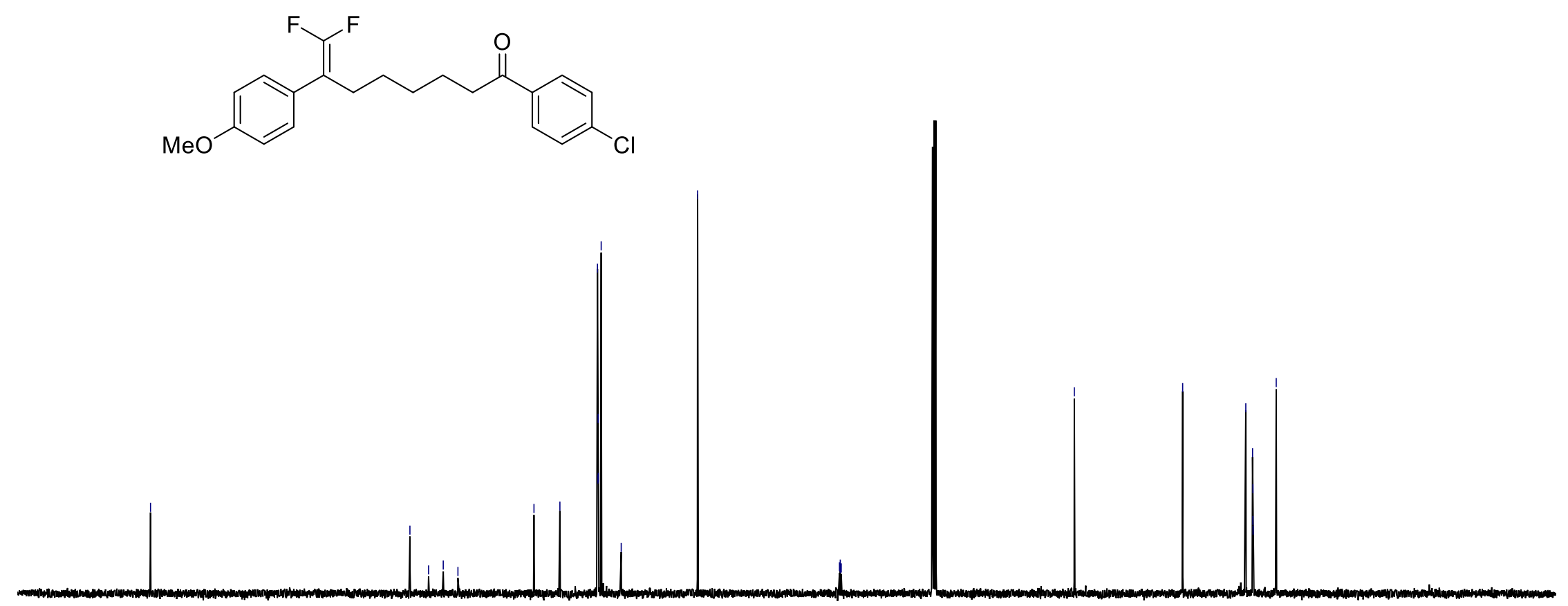

$\begin{array}{llllllllllllllllllllllll}210 & 200 & 190 & 180 & 170 & 160 & 150 & 140 & 130 & 120 & \begin{array}{c}110 \\ \mathrm{f} 1(\mathrm{ppm})\end{array}\left(\begin{array}{ll}100 \\ (\mathrm{pmm})\end{array}\right. & 80 & 70 & 60 & 50 & 40 & 30 & 20 & 10 & 0 & -10\end{array}$ S109 
${ }^{19} \mathrm{~F}$ NMR-spectrum $\left(376 \mathrm{MHz}, \mathrm{CDCl}_{3}\right)$ of $\mathbf{3 a n}$

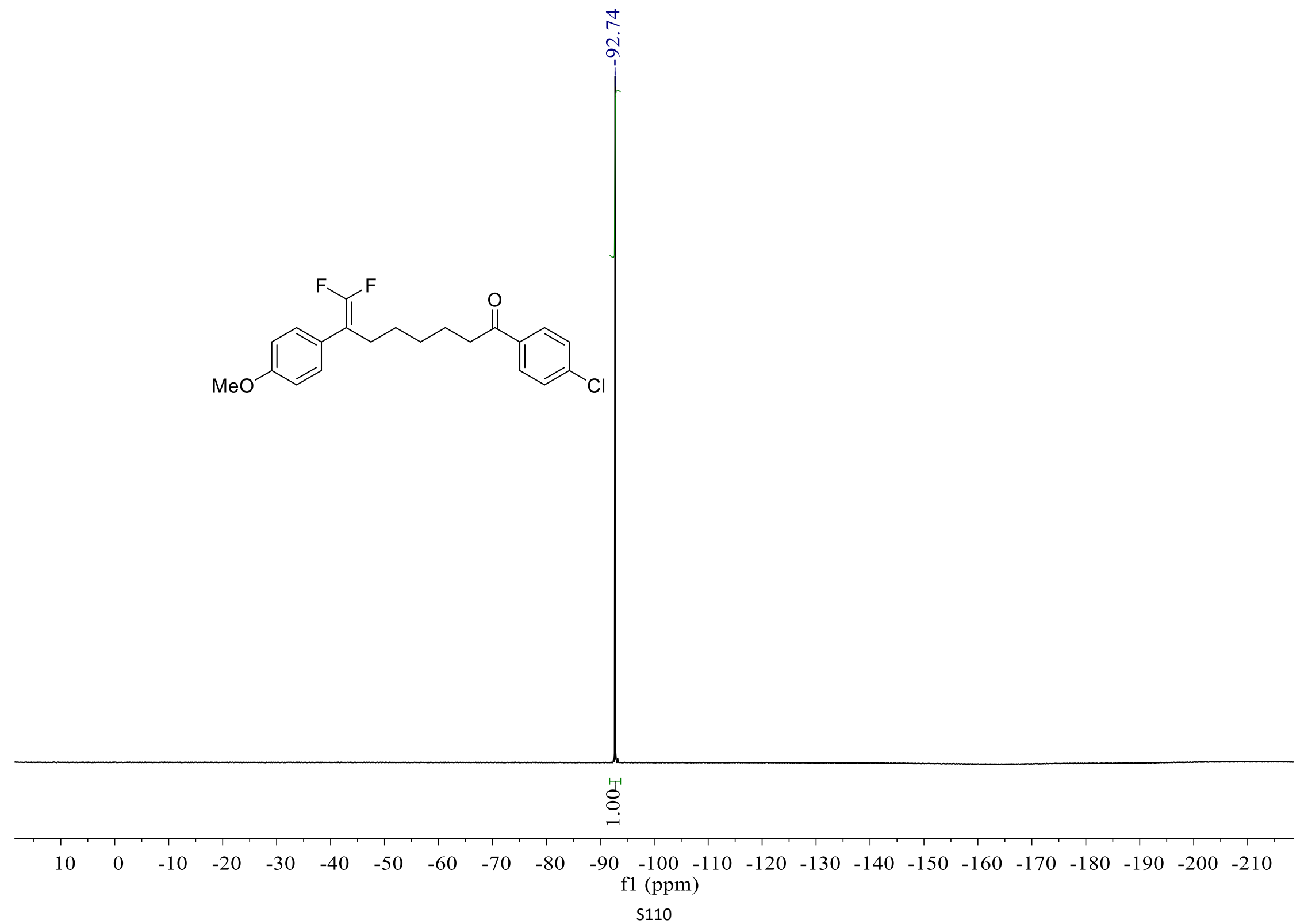




\section{${ }^{1} \mathrm{H}$ NMR-spectrum (400 MHz, $\mathrm{CDCl}_{3}$ ) of $\mathbf{3 a o}$}

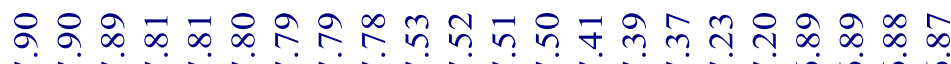

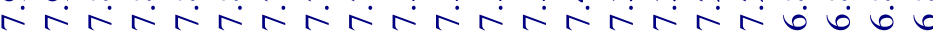

\& $\quad \overrightarrow{0}$ a

$>>$
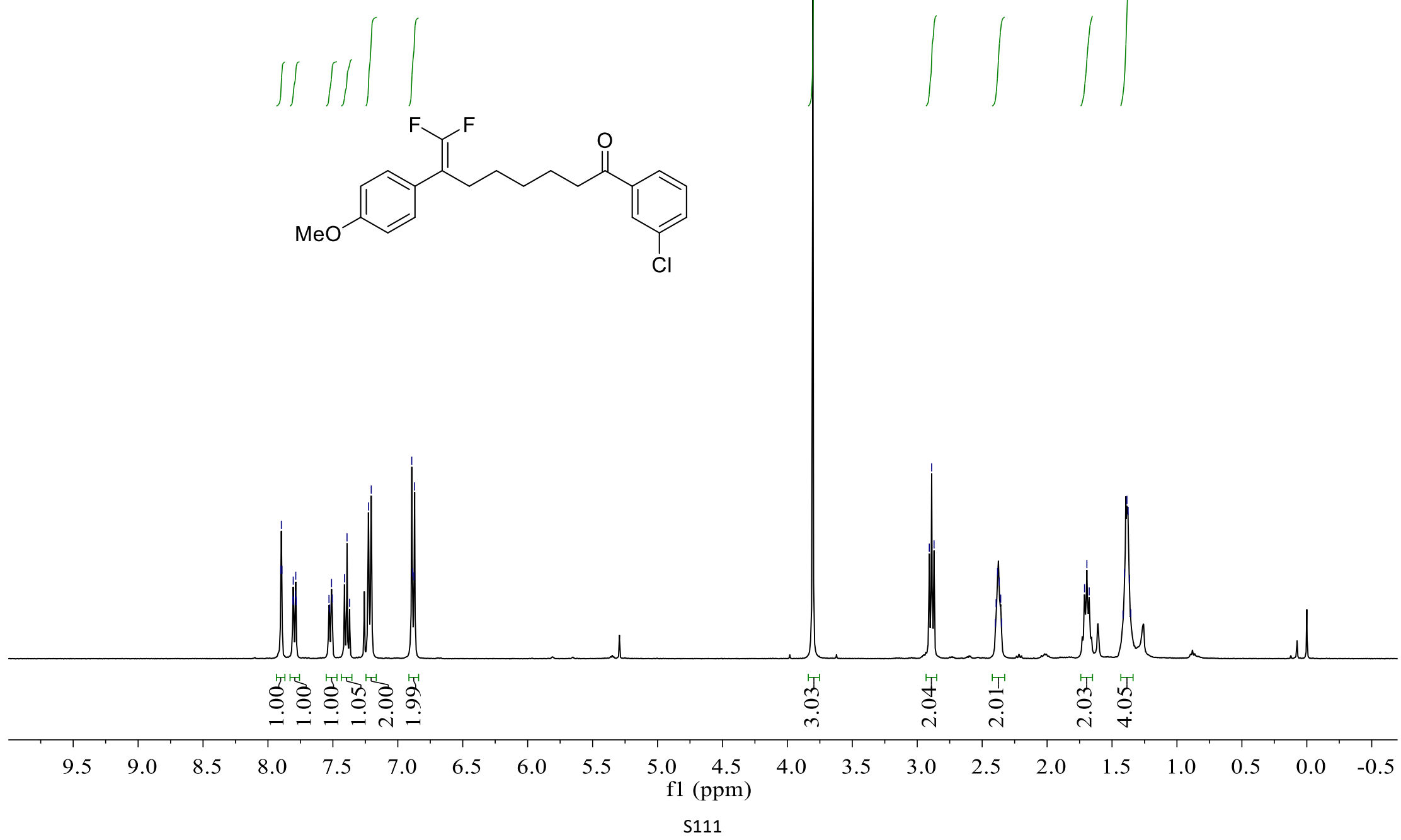

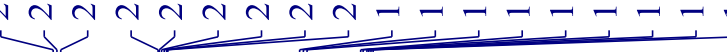


${ }^{13} \mathrm{C}\left\{{ }^{1} \mathrm{H}\right\}$ NMR-spectrum $\left(126 \mathrm{MHz}, \mathrm{CDCl}_{3}\right)$ of $\mathbf{3 a o}$

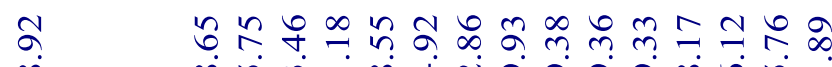

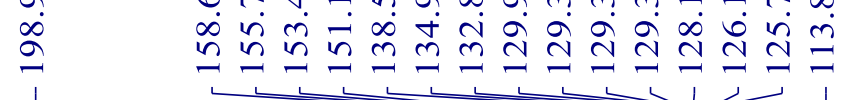

शิ

बंबेंब

กูก

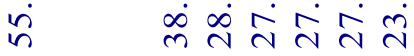

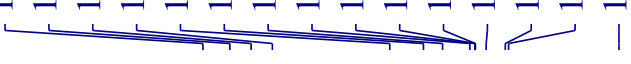

$\sqrt{n+4}$

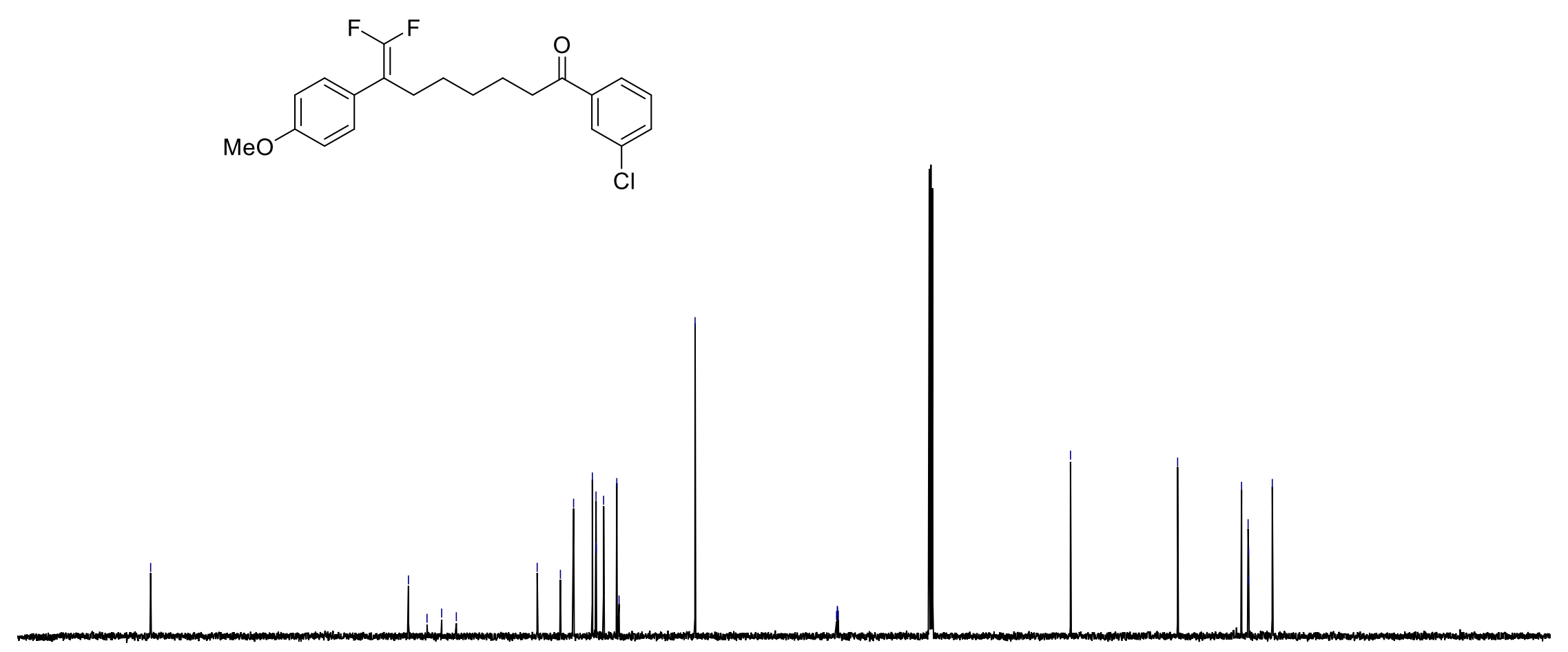

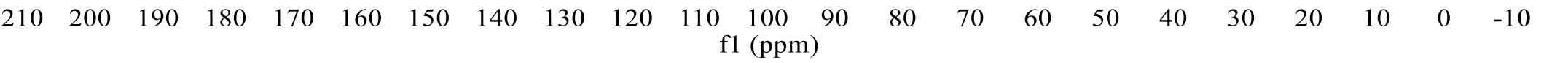
S112 
${ }^{19} \mathrm{~F}$ NMR-spectrum $\left(376 \mathrm{MHz}, \mathrm{CDCl}_{3}\right)$ of $\mathbf{3 a o}$

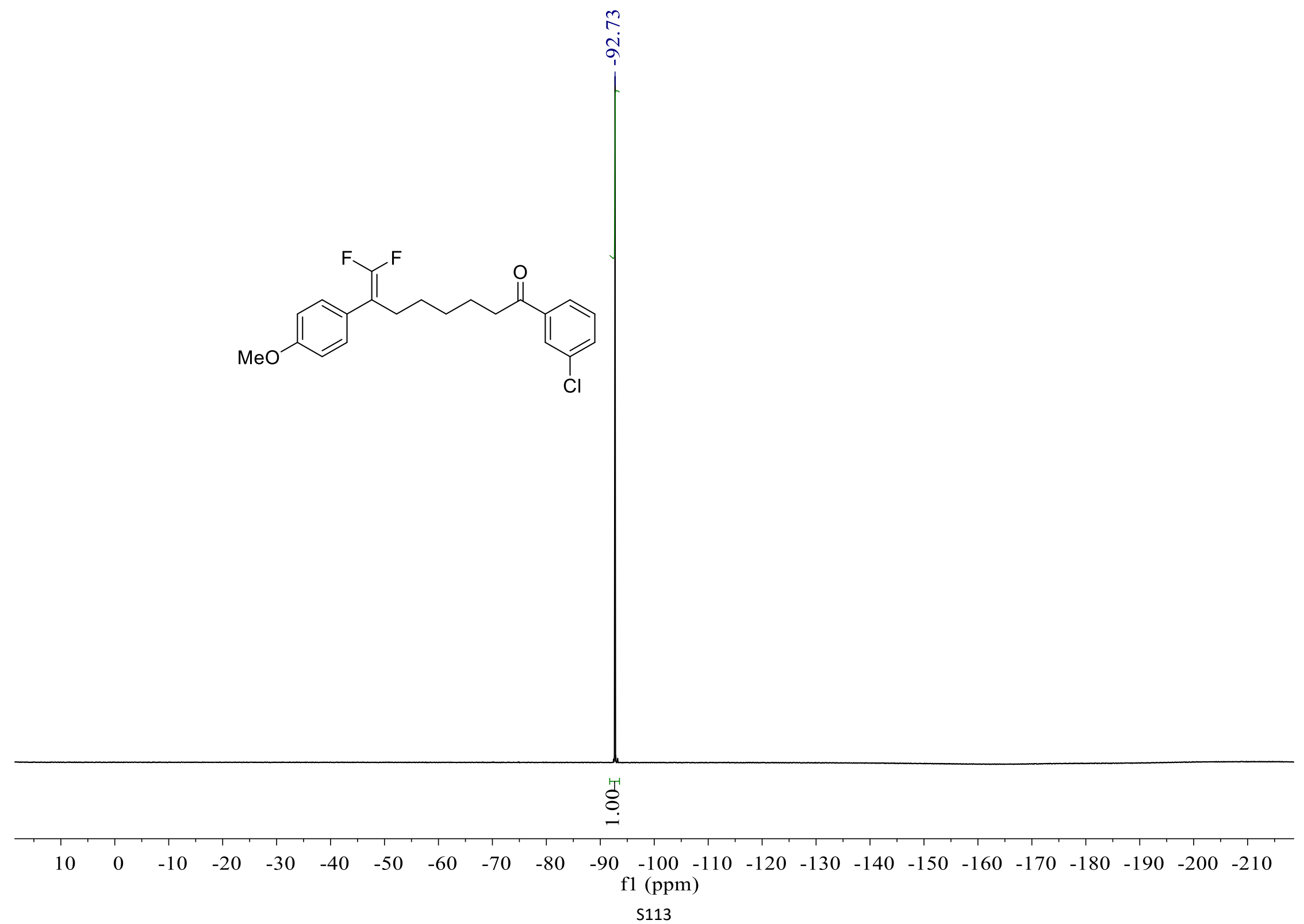




\section{${ }^{1} \mathrm{H}$ NMR-spectrum $\left(400 \mathrm{MHz}, \mathrm{CDCl}_{3}\right)$ of $\mathbf{3 a p}$}

ถูำ

in

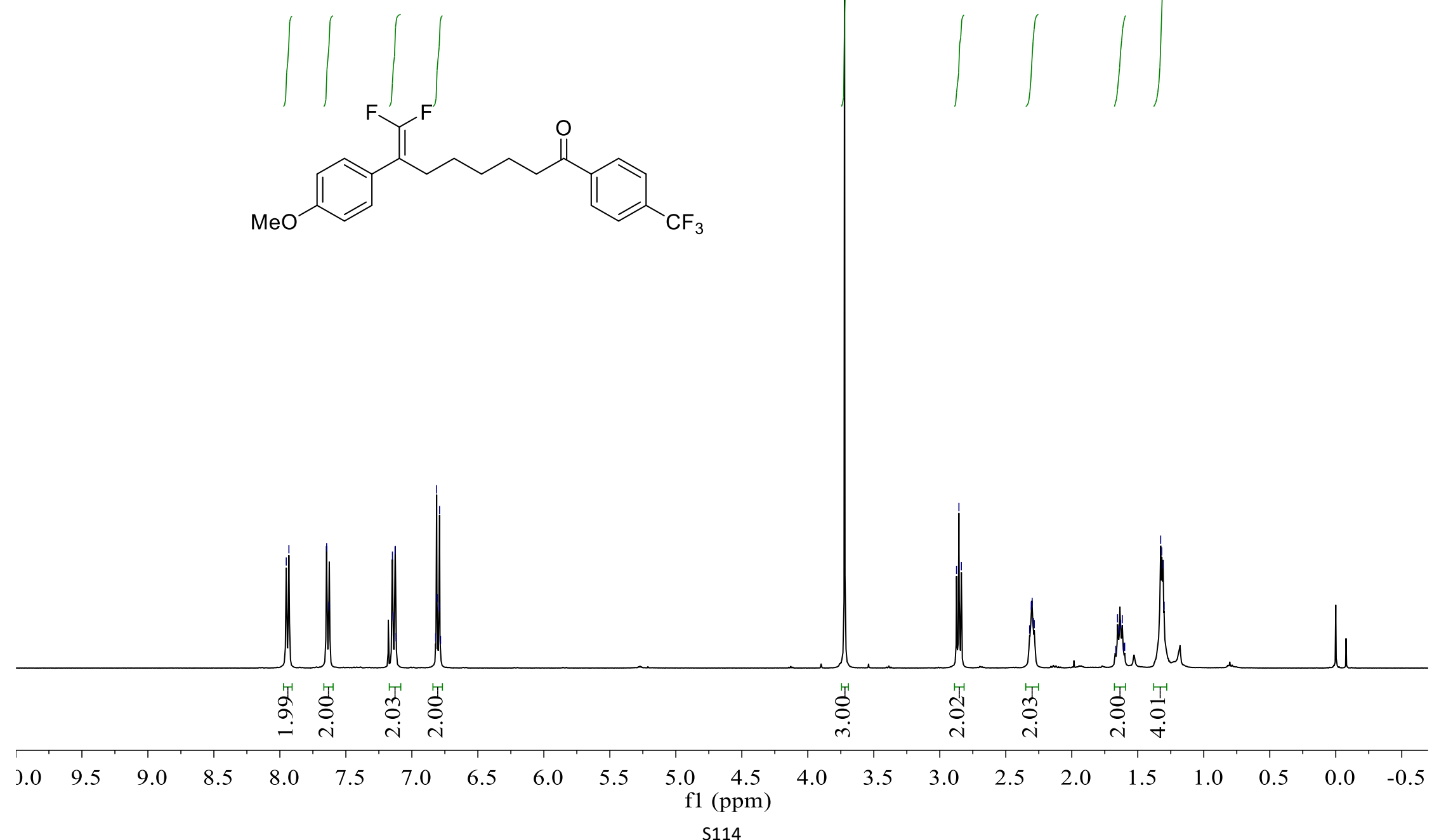

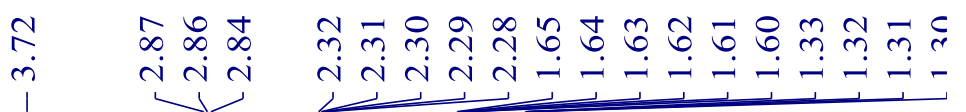

12

S114 
${ }^{13} \mathrm{C}\left\{{ }^{1} \mathrm{H}\right\}$ NMR-spectrum $\left(126 \mathrm{MHz}, \mathrm{CDCl}_{3}\right)$ of 3ap

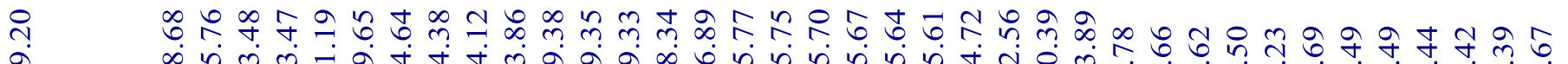

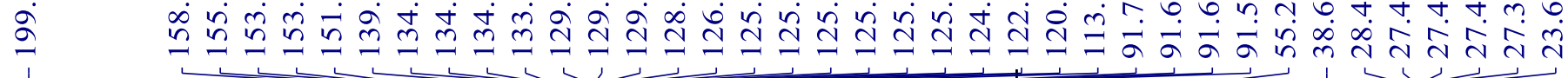

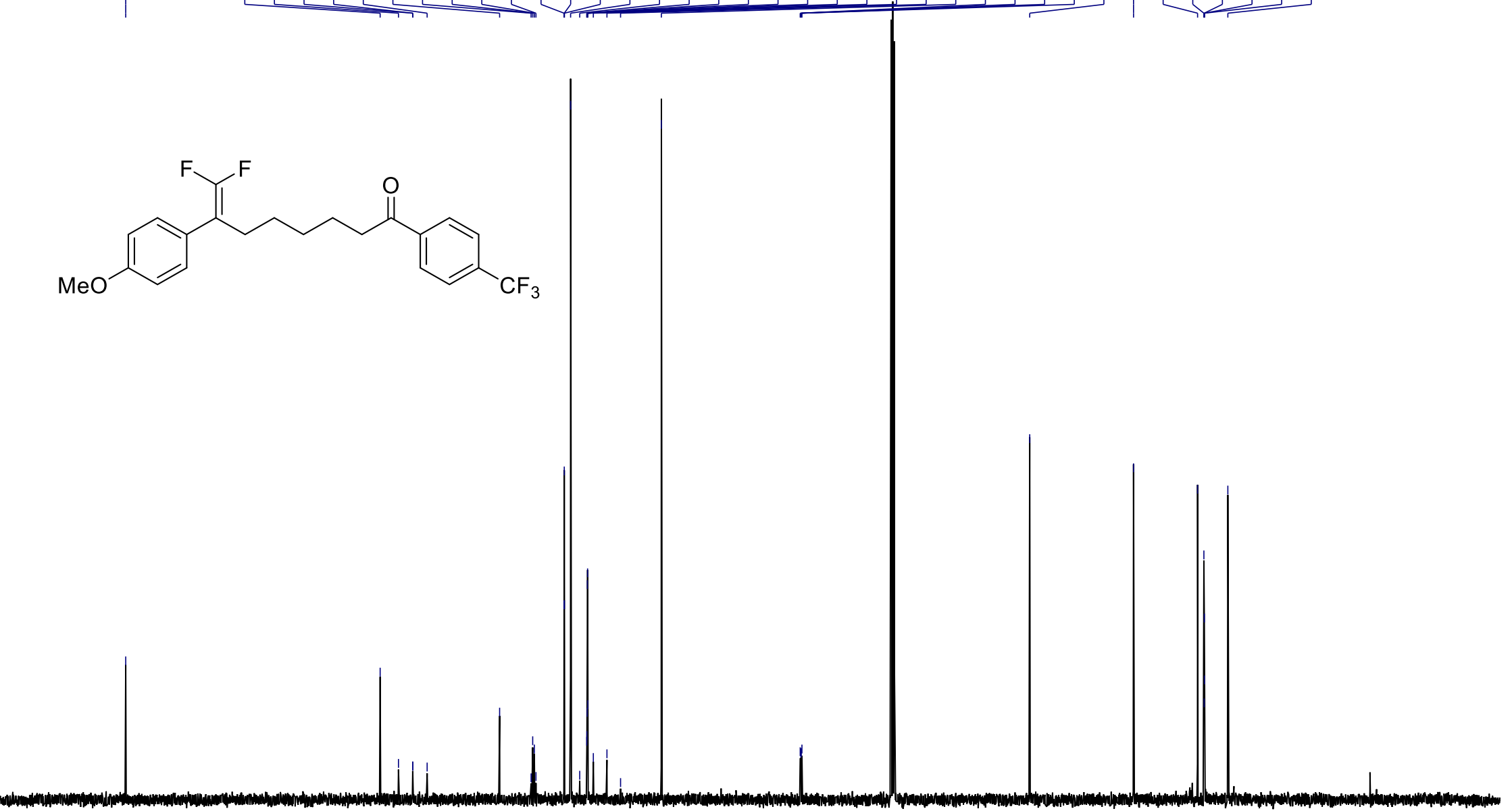

$\begin{array}{llllllllllllllllllllllll}210 & 200 & 190 & 180 & 170 & 160 & 150 & 140 & 130 & 120 & 110 & 100 & 90 & 80 & 70 & 60 & 50 & 40 & 30 & 20 & 10 & 0 & -10\end{array}$ S115 
${ }^{19} \mathrm{~F}$ NMR-spectrum $\left(376 \mathrm{MHz}, \mathrm{CDCl}_{3}\right.$ ) of 3ap

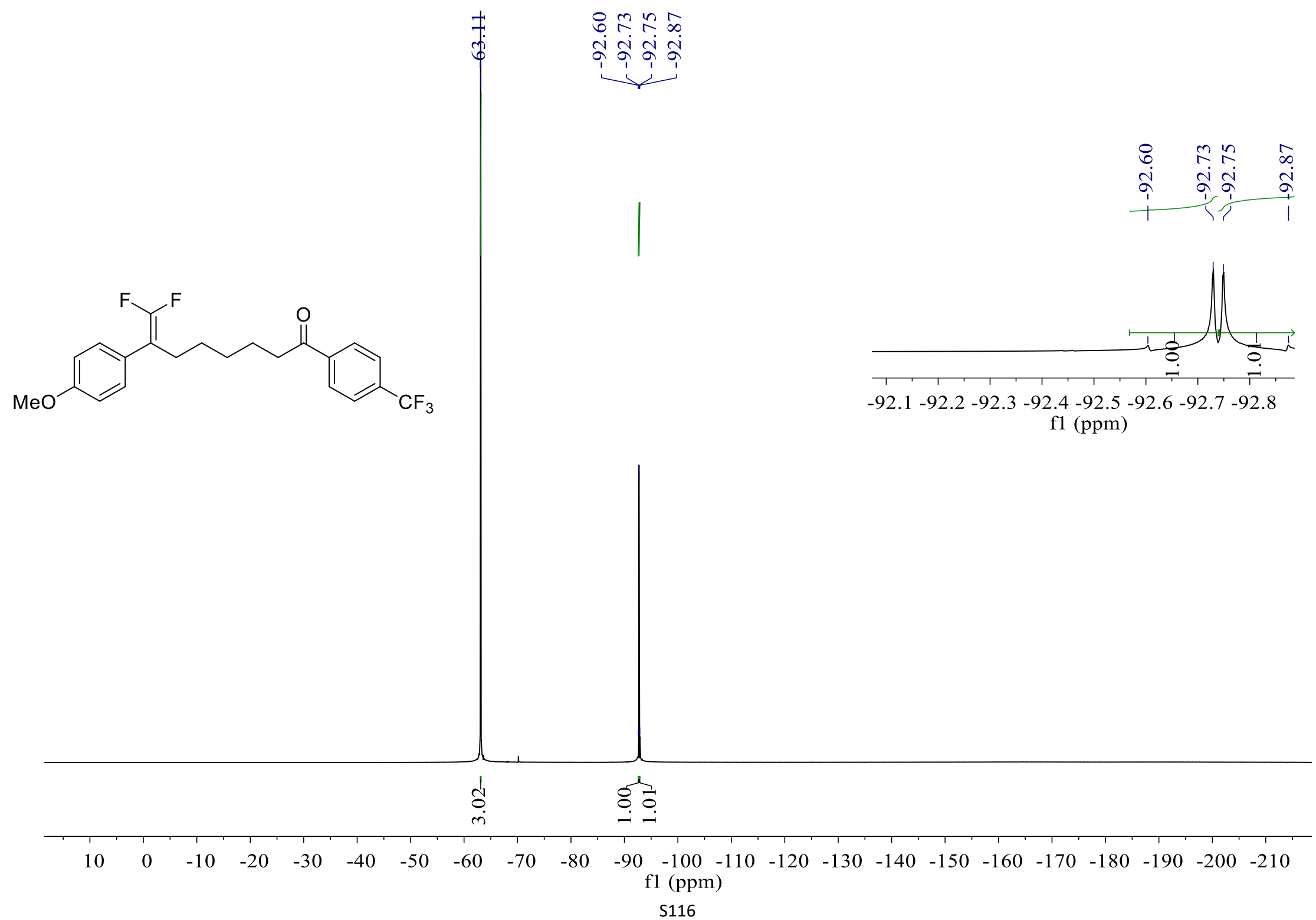




\section{${ }^{1} \mathrm{H}$ NMR-spectrum $\left(500 \mathrm{MHz}, \mathrm{CDCl}_{3}\right.$ ) of $\mathbf{3 a q}$}

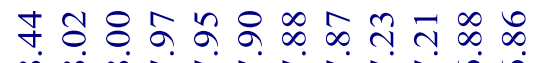

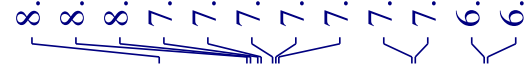
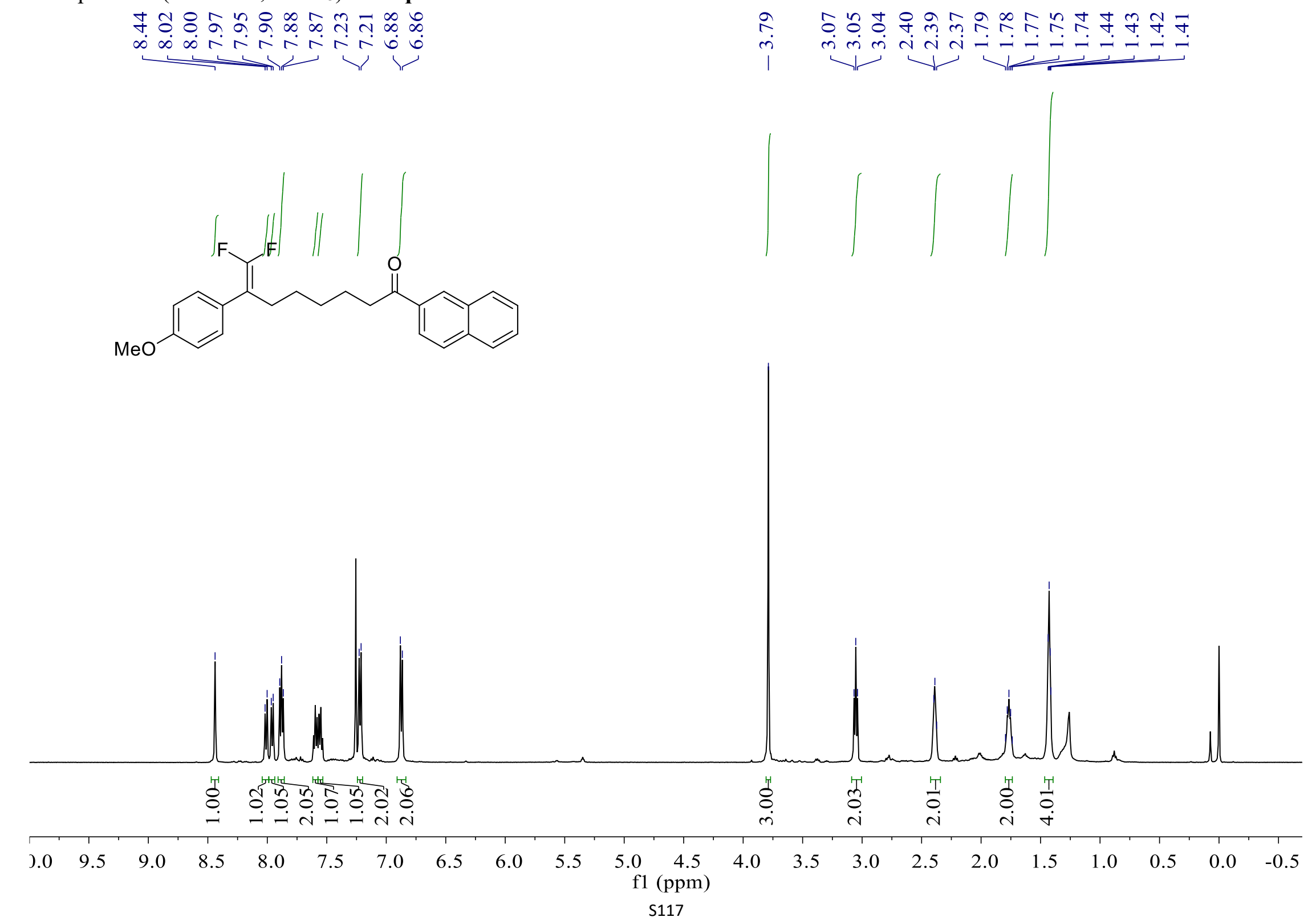
${ }^{13} \mathrm{C}\left\{{ }^{1} \mathrm{H}\right\}$ NMR-spectrum $\left(126 \mathrm{MHz}, \mathrm{CDCl}_{3}\right)$ of $\mathbf{3 a p}$

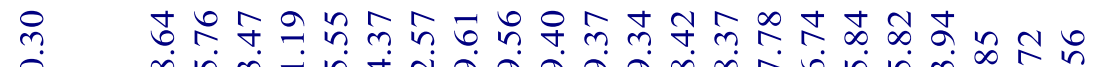

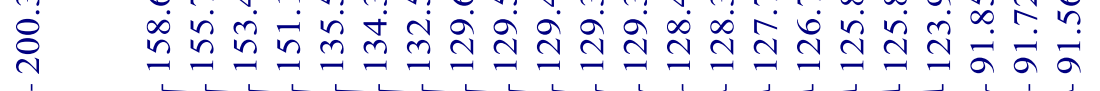
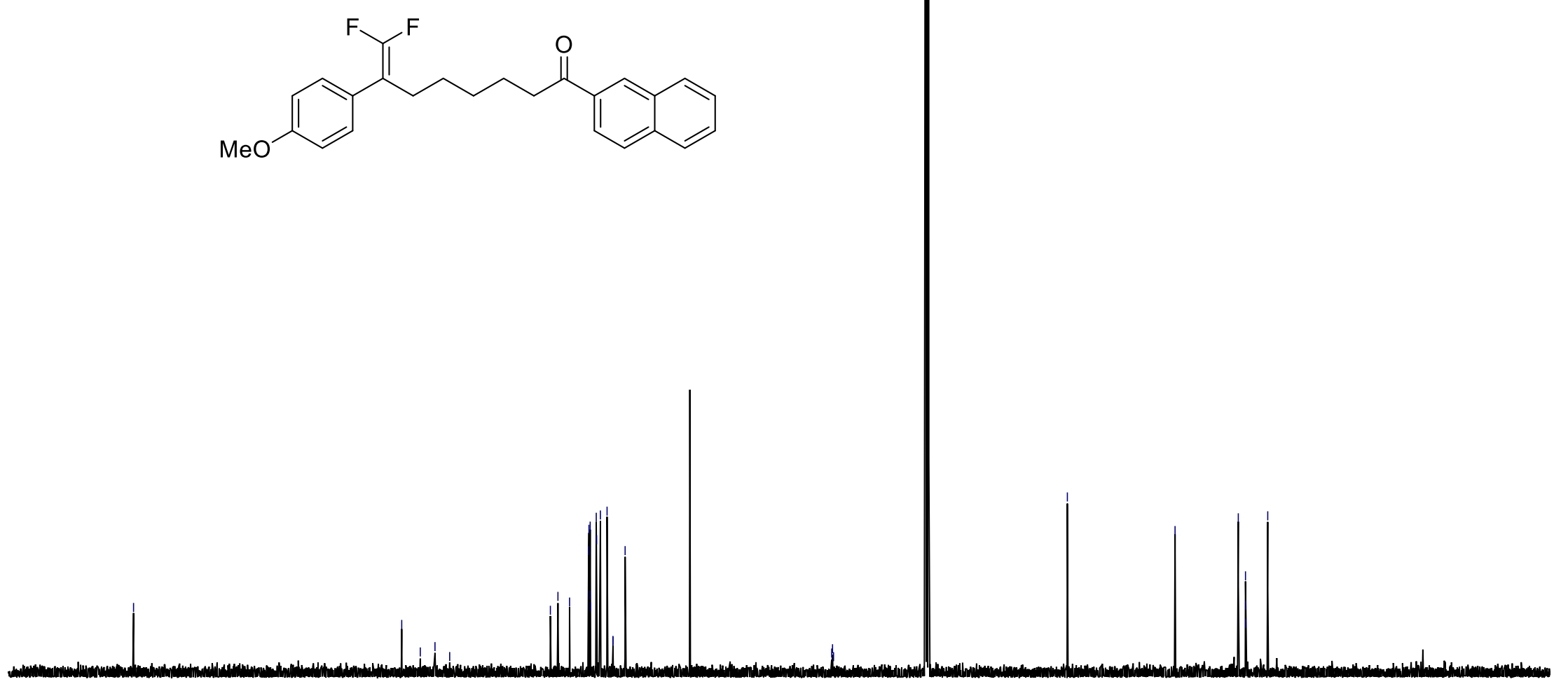

$\begin{array}{lllllllllllll}210 & 200 & 190 & 180 & 170 & 160 & 150 & 140 & 130 & 120 & 110 & 100 & 90\end{array}$

f1 (ppm)

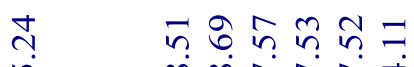

in $\quad m \infty i$

1 
${ }^{19} \mathrm{~F}$ NMR-spectrum $\left(471 \mathrm{MHz}, \mathrm{CDCl}_{3}\right.$ ) of $\mathbf{3 a q}$

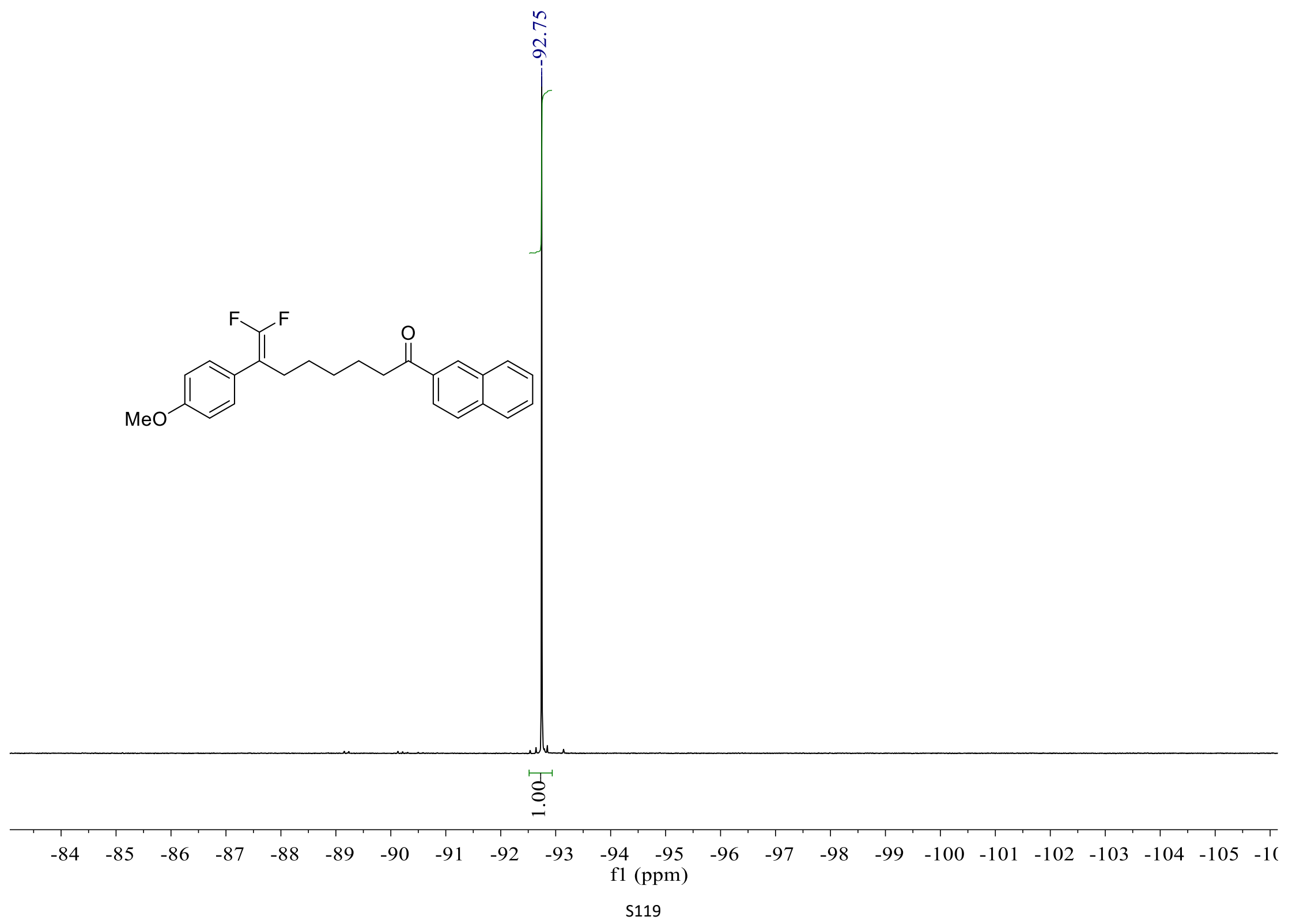




\section{${ }^{1} \mathrm{H}$ NMR-spectrum $\left(500 \mathrm{MHz}, \mathrm{CDCl}_{3}\right)$ of $\mathbf{3 a r}$}

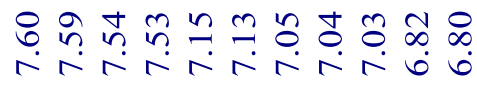

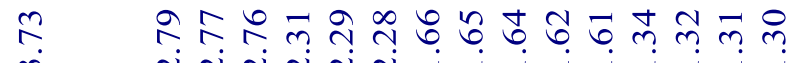

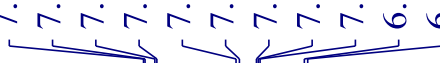

$\dot{1} \quad$ iñ
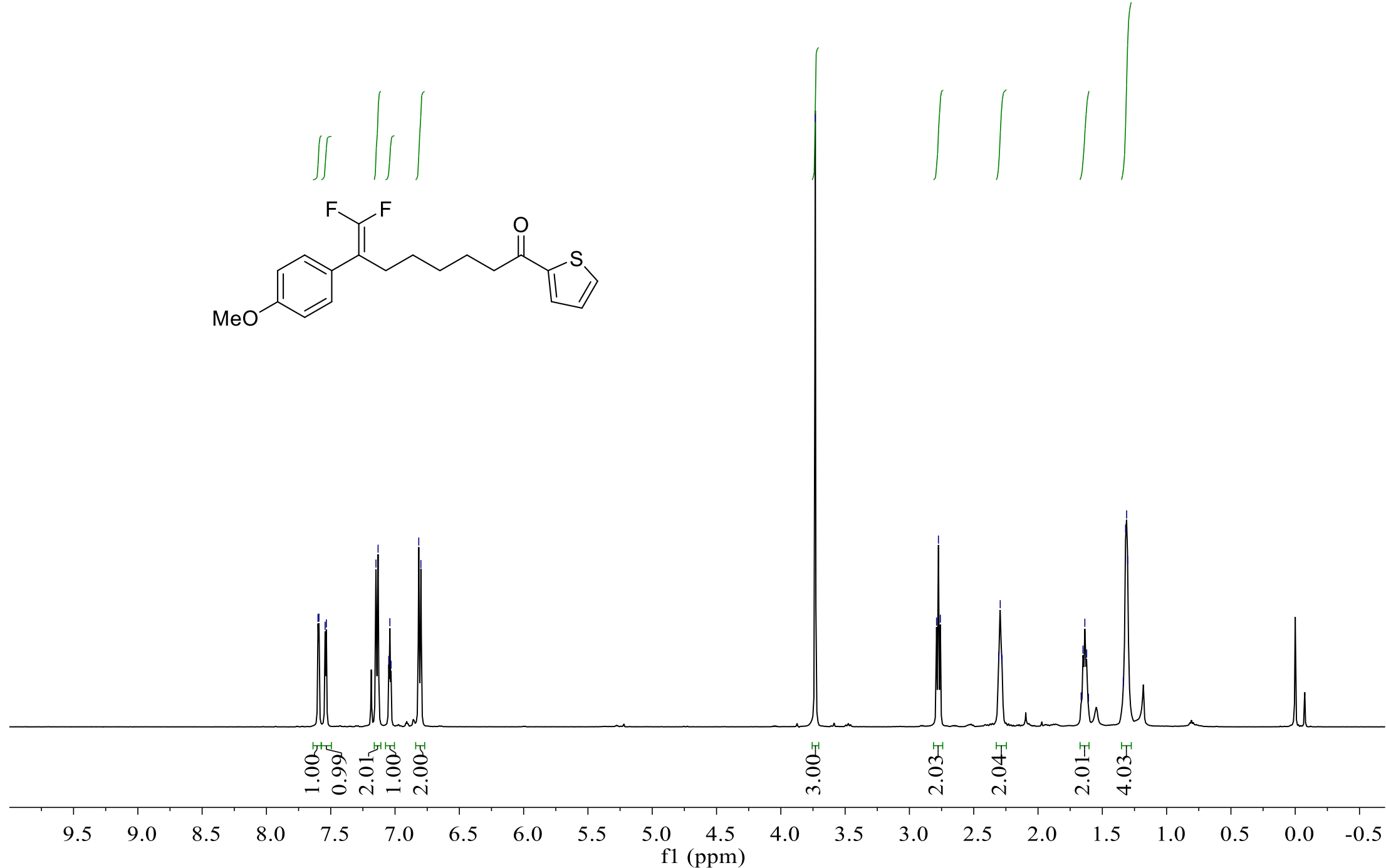
${ }^{13} \mathrm{C}\left\{{ }^{1} \mathrm{H}\right\}$ NMR-spectrum $\left(126 \mathrm{MHz}, \mathrm{CDCl}_{3}\right)$ of $\mathbf{3 a r}$

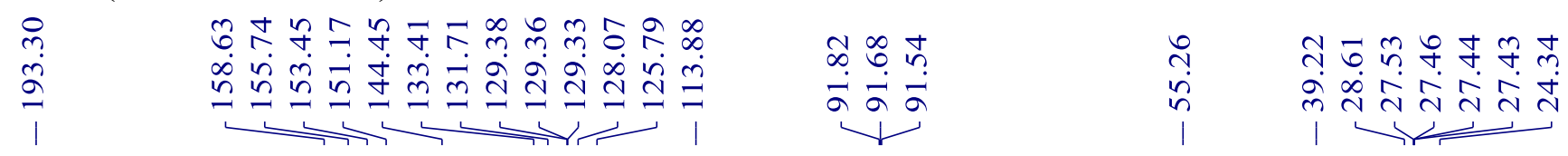

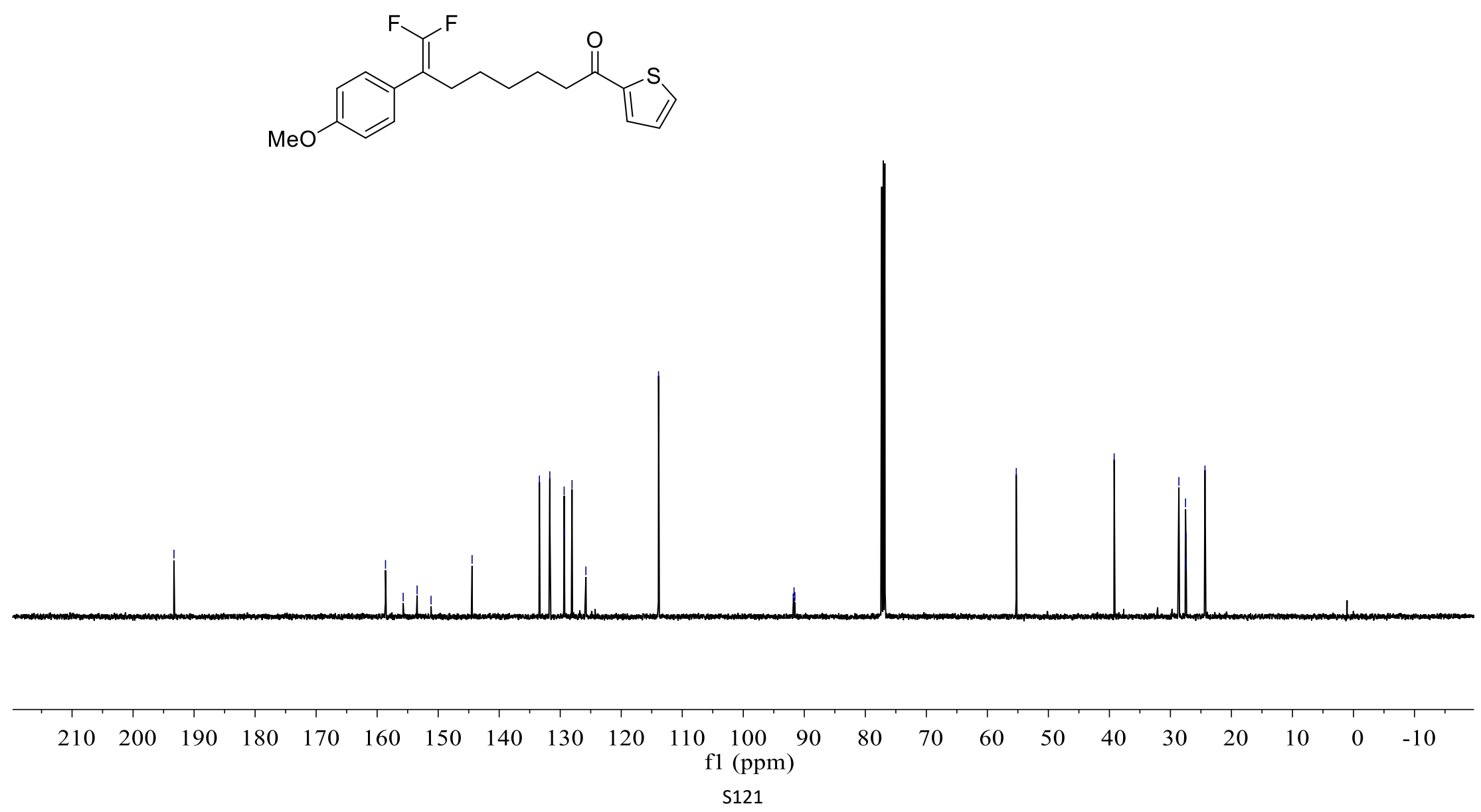


${ }^{19} \mathrm{~F}$ NMR-spectrum $\left(376 \mathrm{MHz}, \mathrm{CDCl}_{3}\right)$ of $\mathbf{3 a r}$

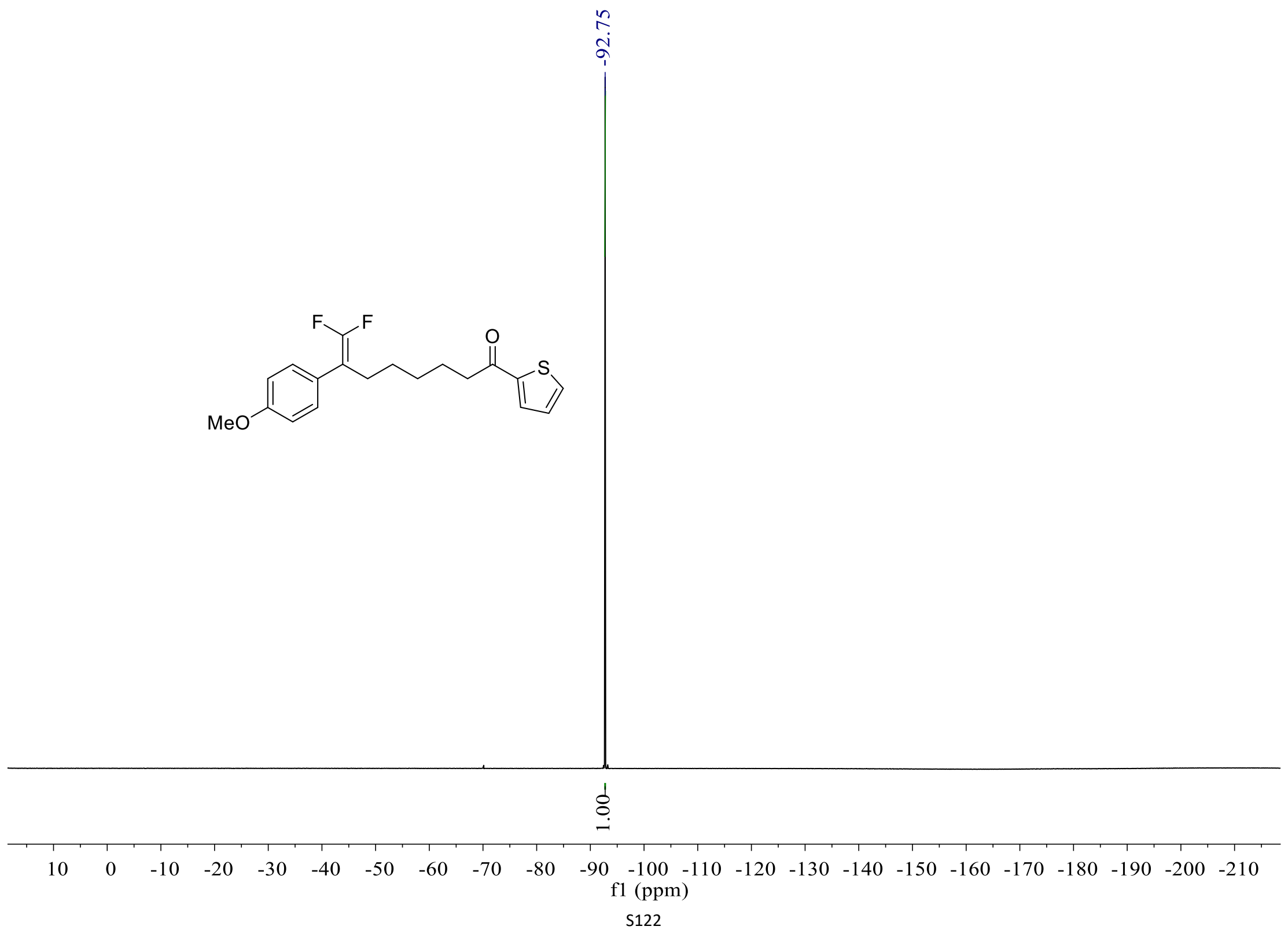




\section{${ }^{1} \mathrm{H}$ NMR-spectrum (500 MHz, $\mathrm{CDCl}_{3}$ ) of 3as}

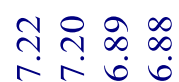

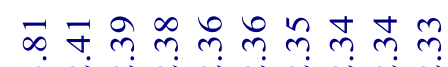

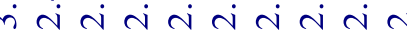

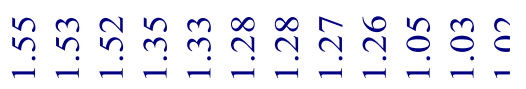

i)
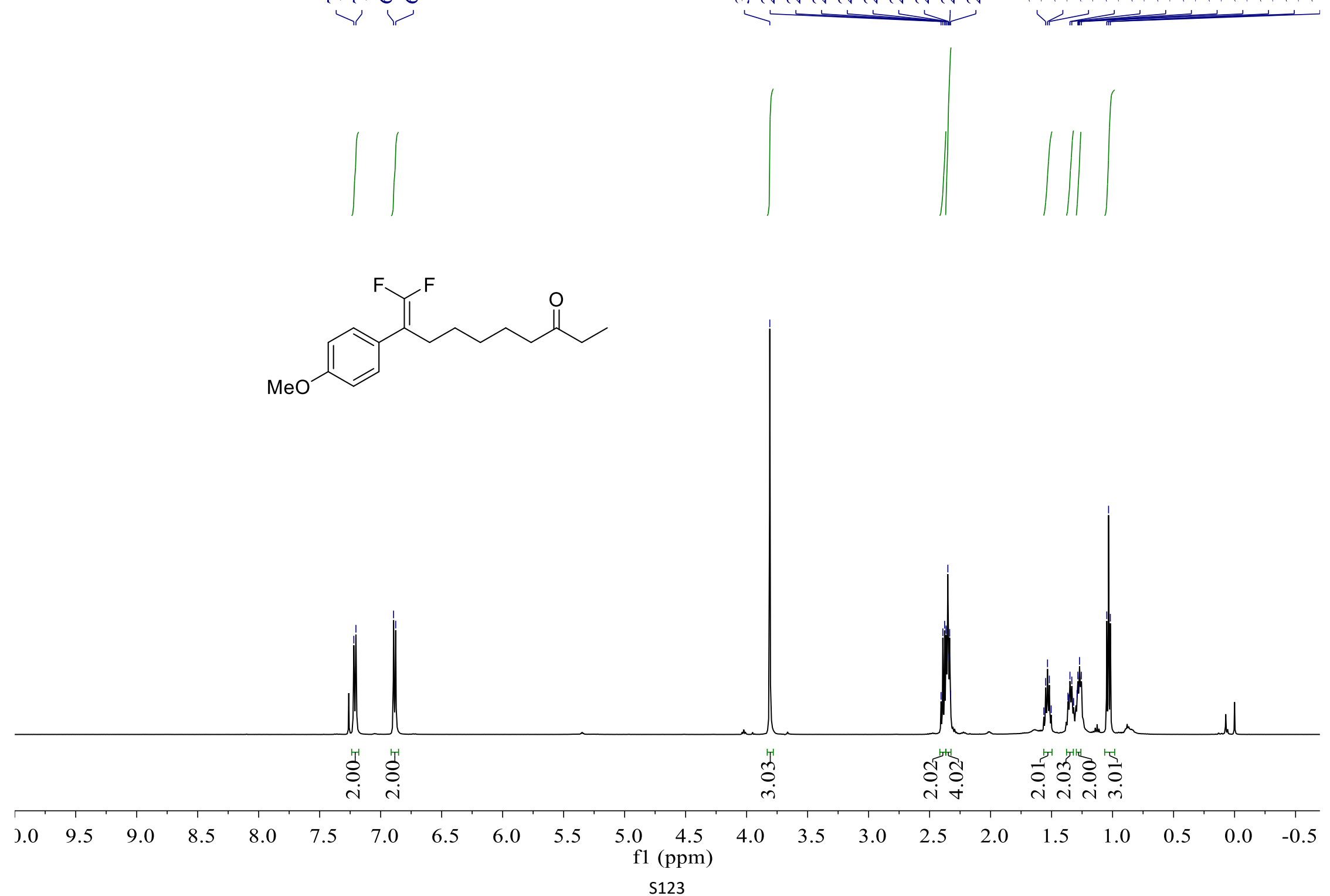
${ }^{13} \mathrm{C}\left\{{ }^{1} \mathrm{H}\right\}$ NMR-spectrum $\left(126 \mathrm{MHz}, \mathrm{CDCl}_{3}\right)$ of 3as

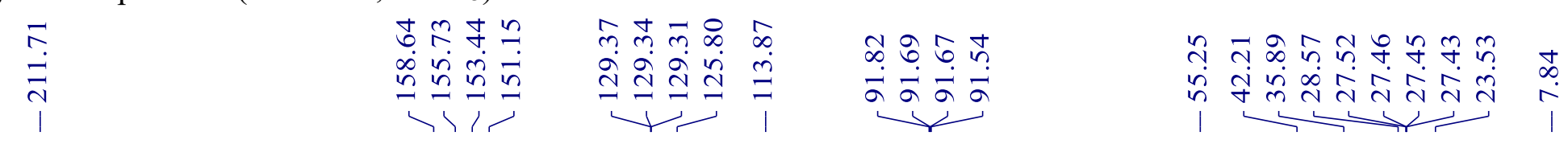

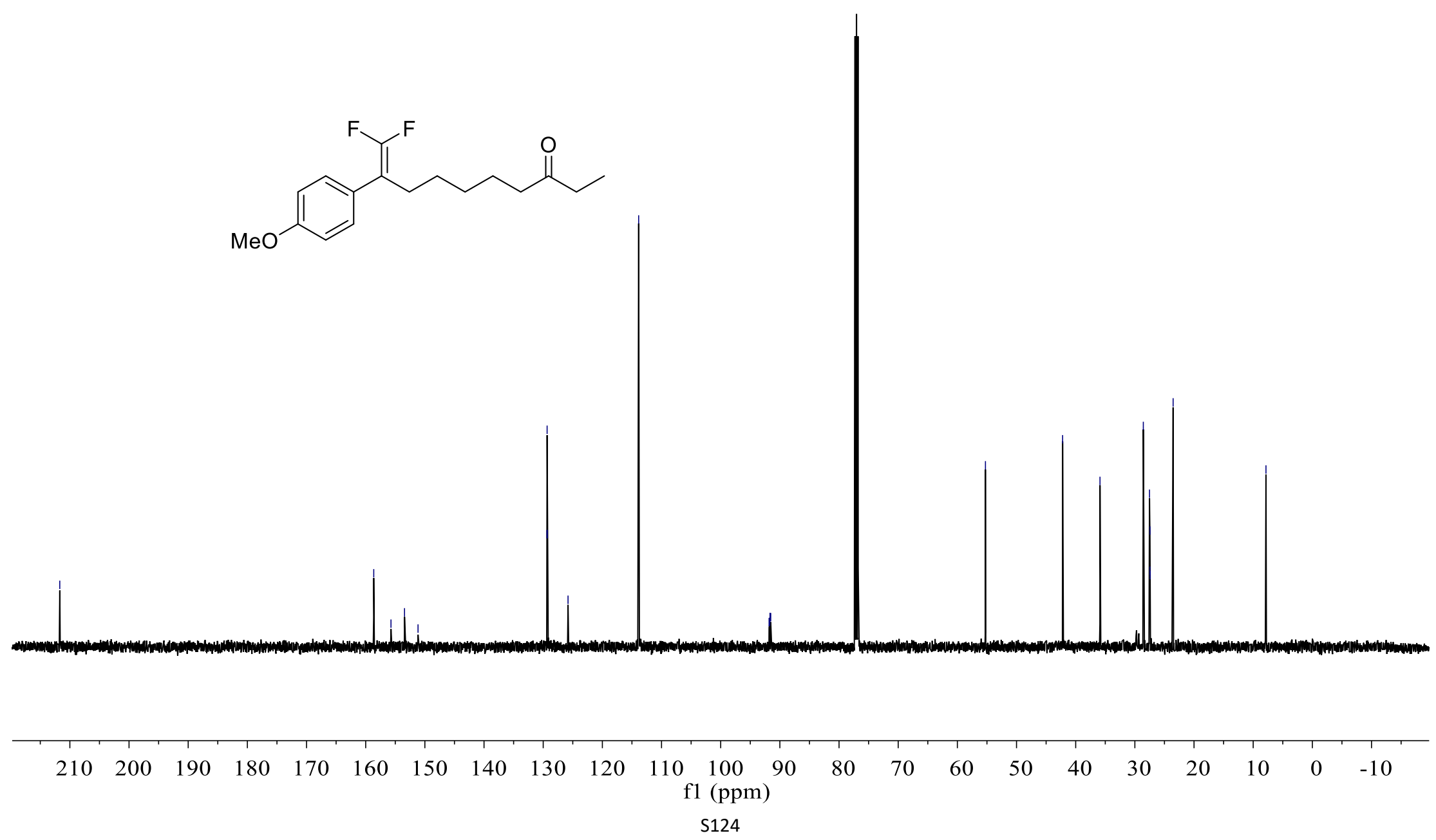


${ }^{19} \mathrm{~F}$ NMR-spectrum $\left(471 \mathrm{MHz}, \mathrm{CDCl}_{3}\right)$ of 3as

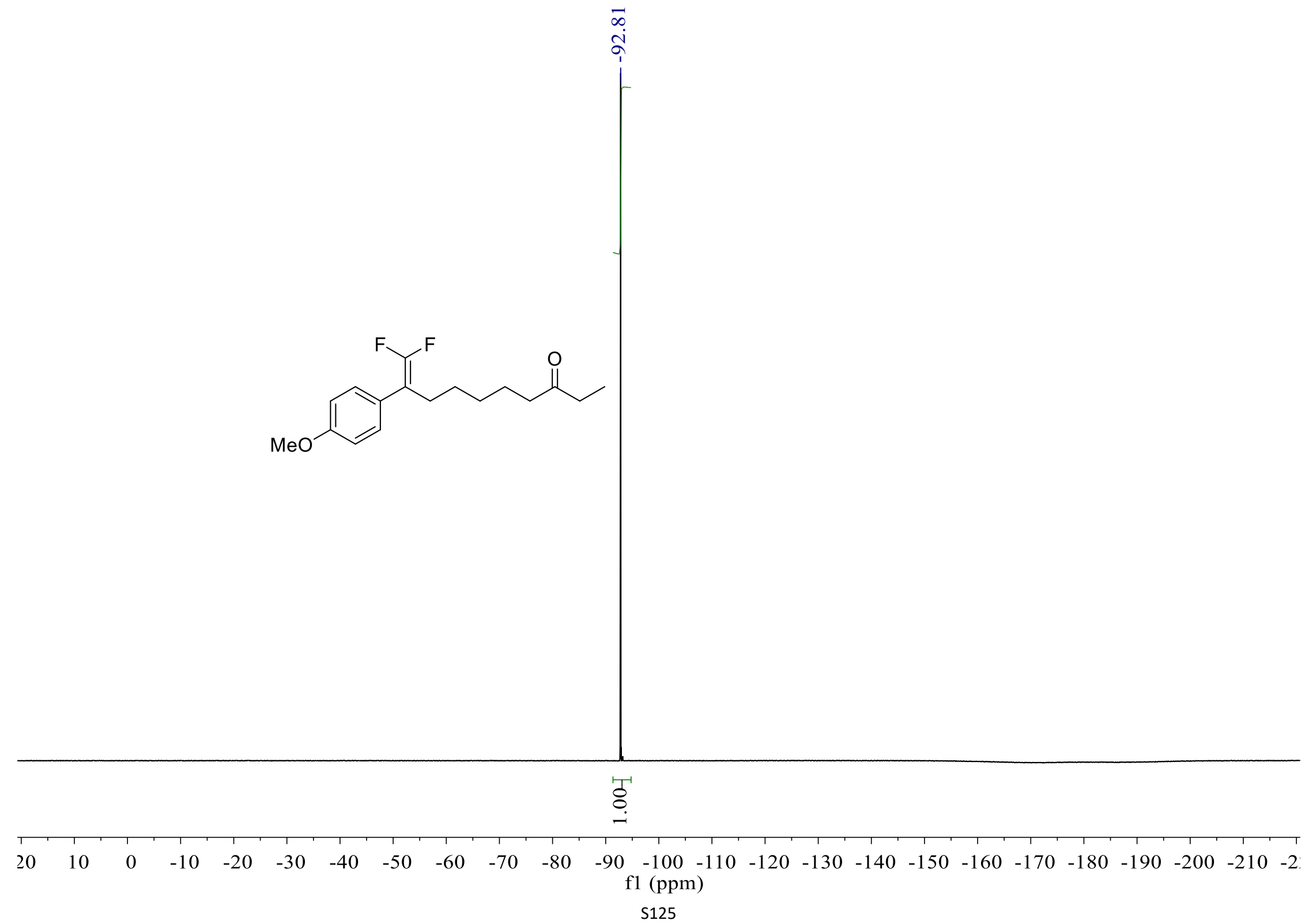




\section{${ }^{1} \mathrm{H}$ NMR-spectrum $\left(500 \mathrm{MHz}, \mathrm{CDCl}_{3}\right)$ of $\mathbf{4 a}$}

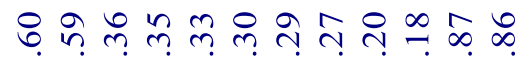

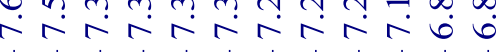

(1)

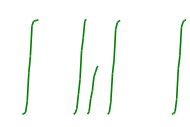

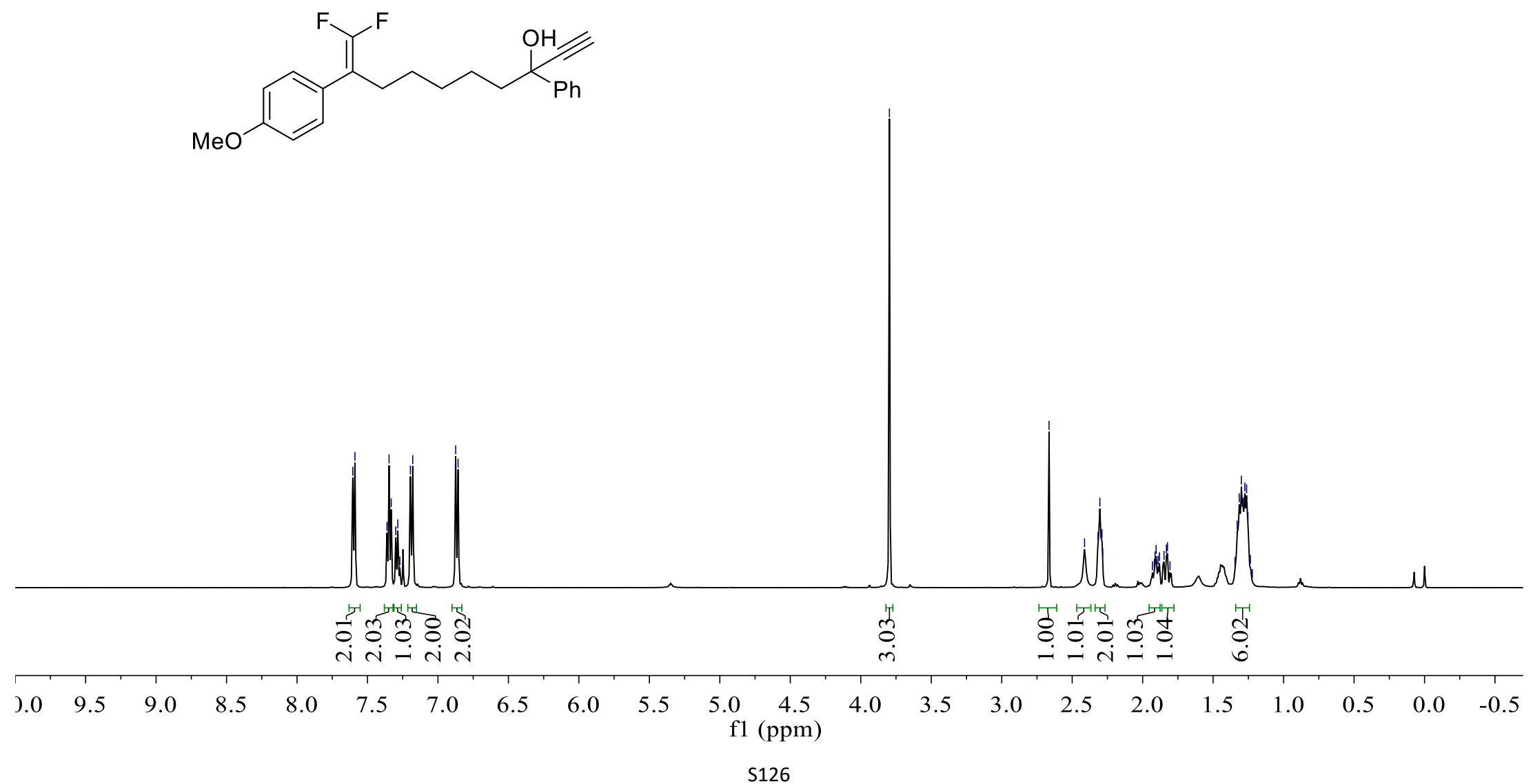

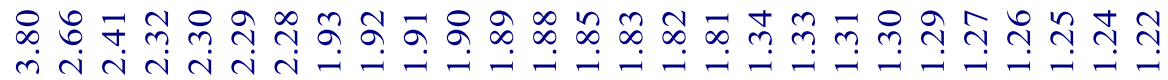

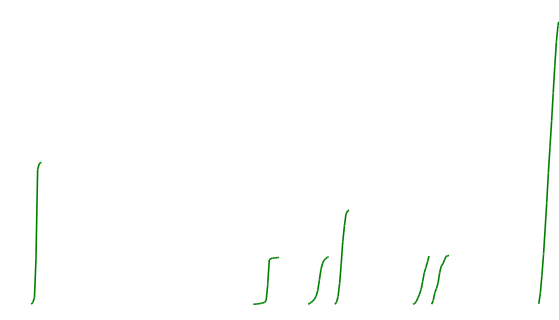


${ }^{13} \mathrm{C}\left\{{ }^{1} \mathrm{H}\right\}$ NMR-spectrum $\left(126 \mathrm{MHz}, \mathrm{CDCl}_{3}\right)$ of $\mathbf{4 a}$

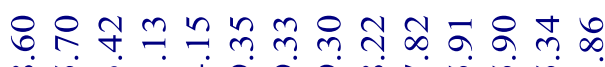

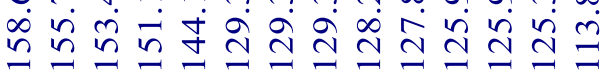

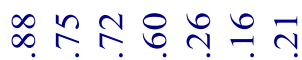

बंबढँ

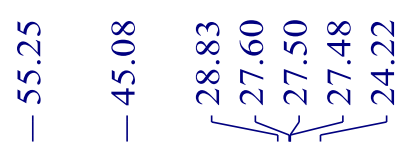

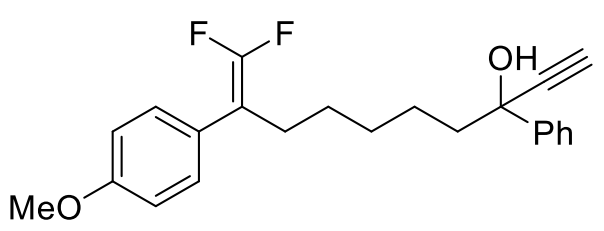

fl (ppm)

S127 
${ }^{19} \mathrm{~F}$ NMR-spectrum $\left(376 \mathrm{MHz}, \mathrm{CDCl}_{3}\right)$ of $\mathbf{4 a}$

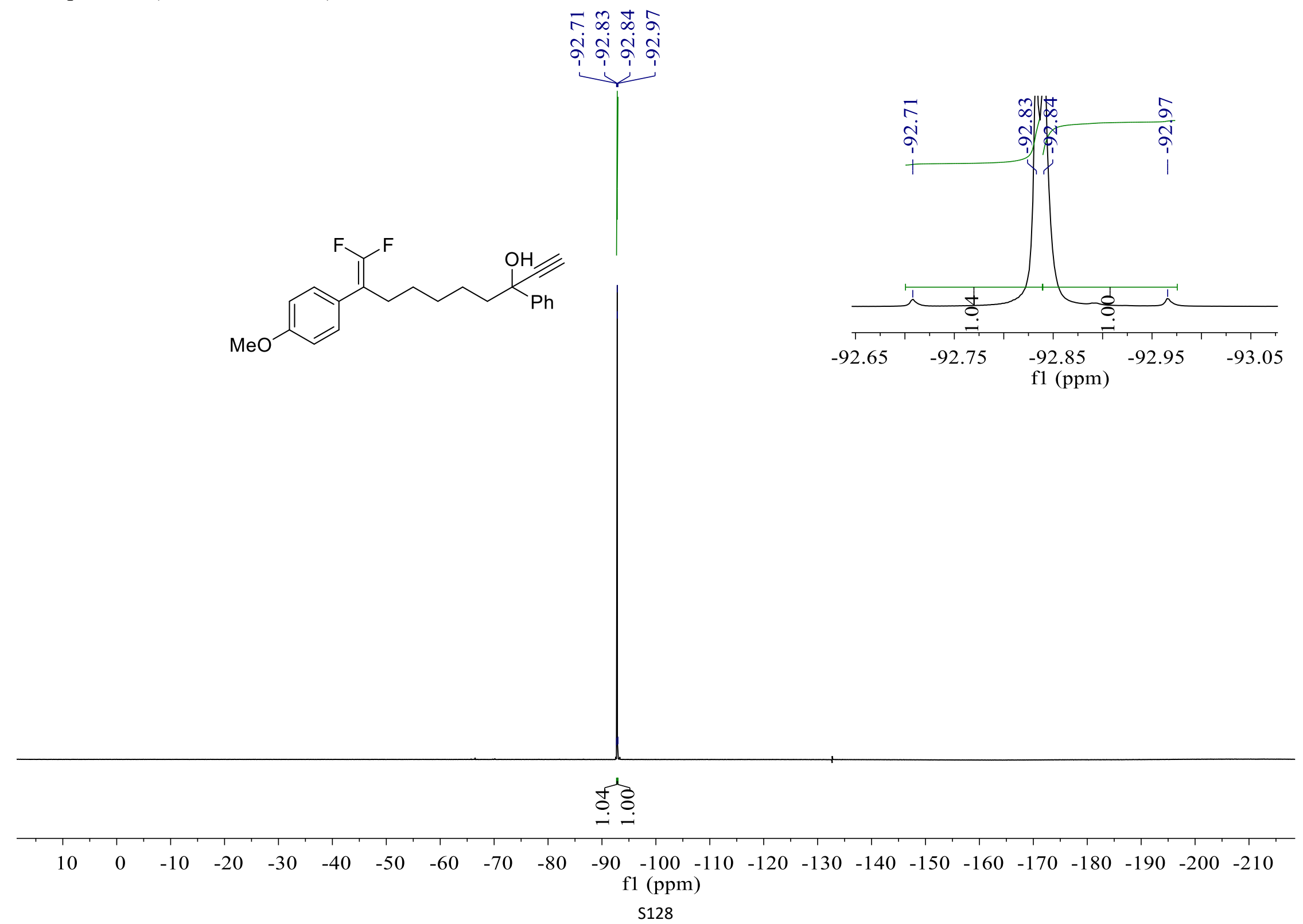




\section{${ }^{1} \mathrm{H}$ NMR-spectrum $\left(400 \mathrm{MHz}, \mathrm{CDCl}_{3}\right)$ of $\mathbf{4 b}$}

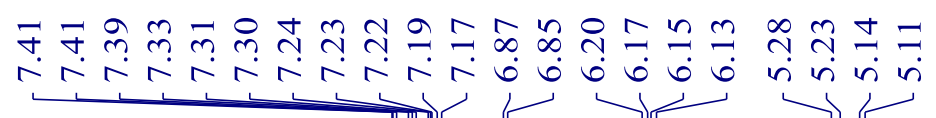

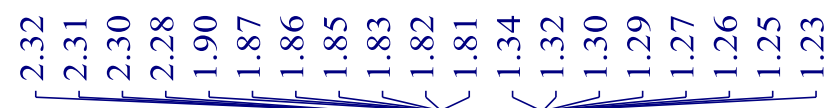

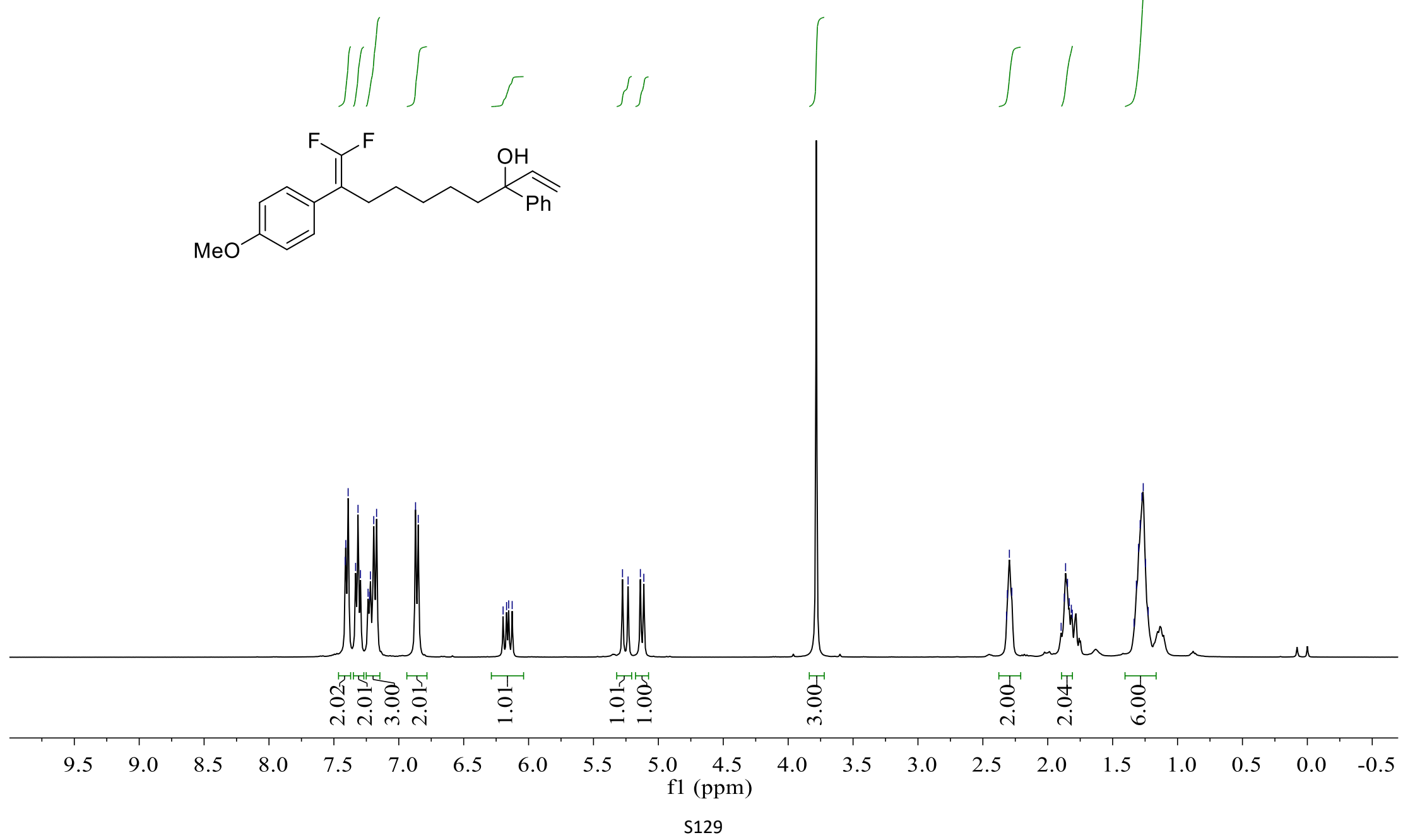


${ }^{13} \mathrm{C}\left\{{ }^{1} \mathrm{H}\right\}$ NMR-spectrum $\left(126 \mathrm{MHz}, \mathrm{CDCl}_{3}\right)$ of $\mathbf{4 b}$

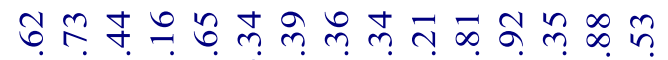

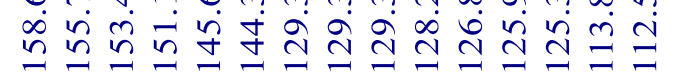

质

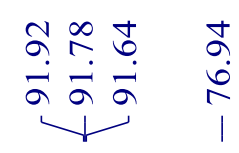

กับ

in
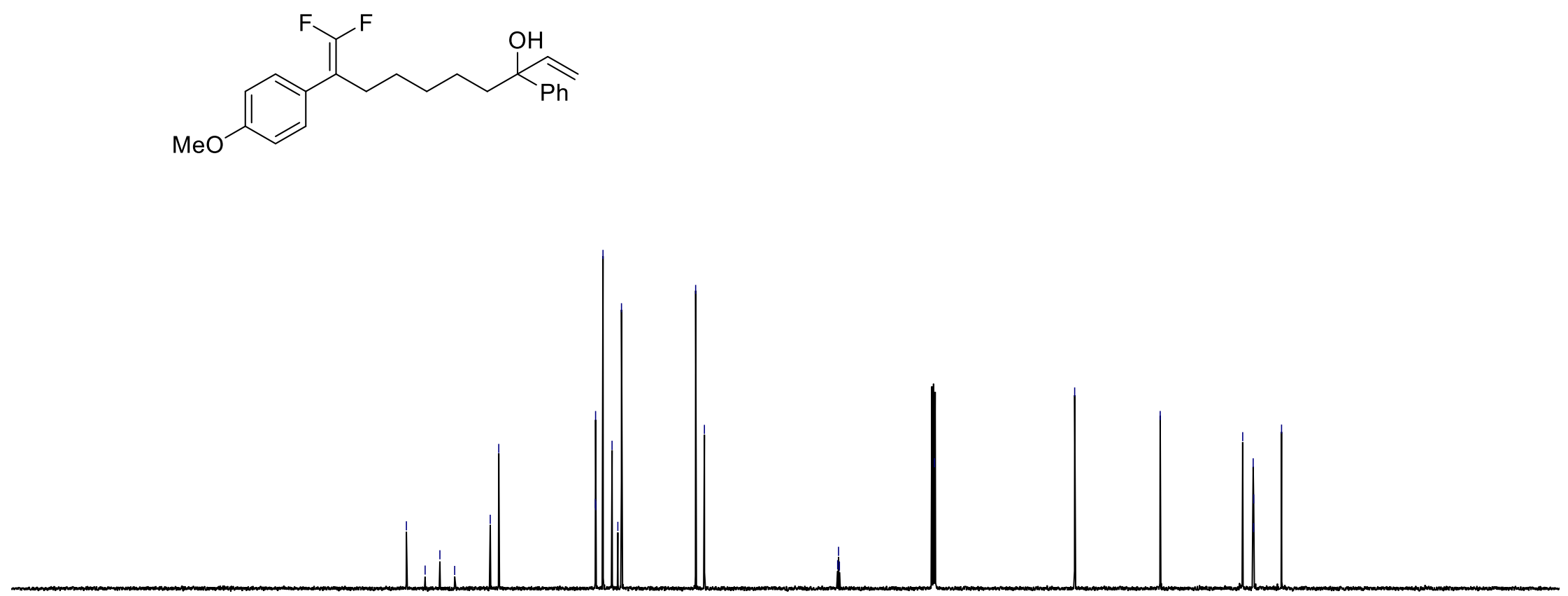

$\begin{array}{llllllllllll}210 & 200 & 190 & 180 & 170 & 160 & 150 & 140 & 130 & 120 & 110 & 100\end{array}$

f1 $(\mathrm{ppm})$

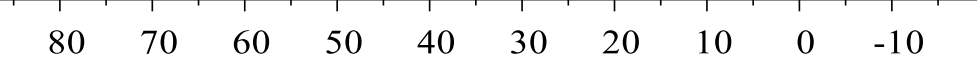

S130 
${ }^{19} \mathrm{~F}$ NMR-spectrum $\left(126 \mathrm{MHz}, \mathrm{CDCl}_{3}\right)$ of $\mathbf{4 b}$

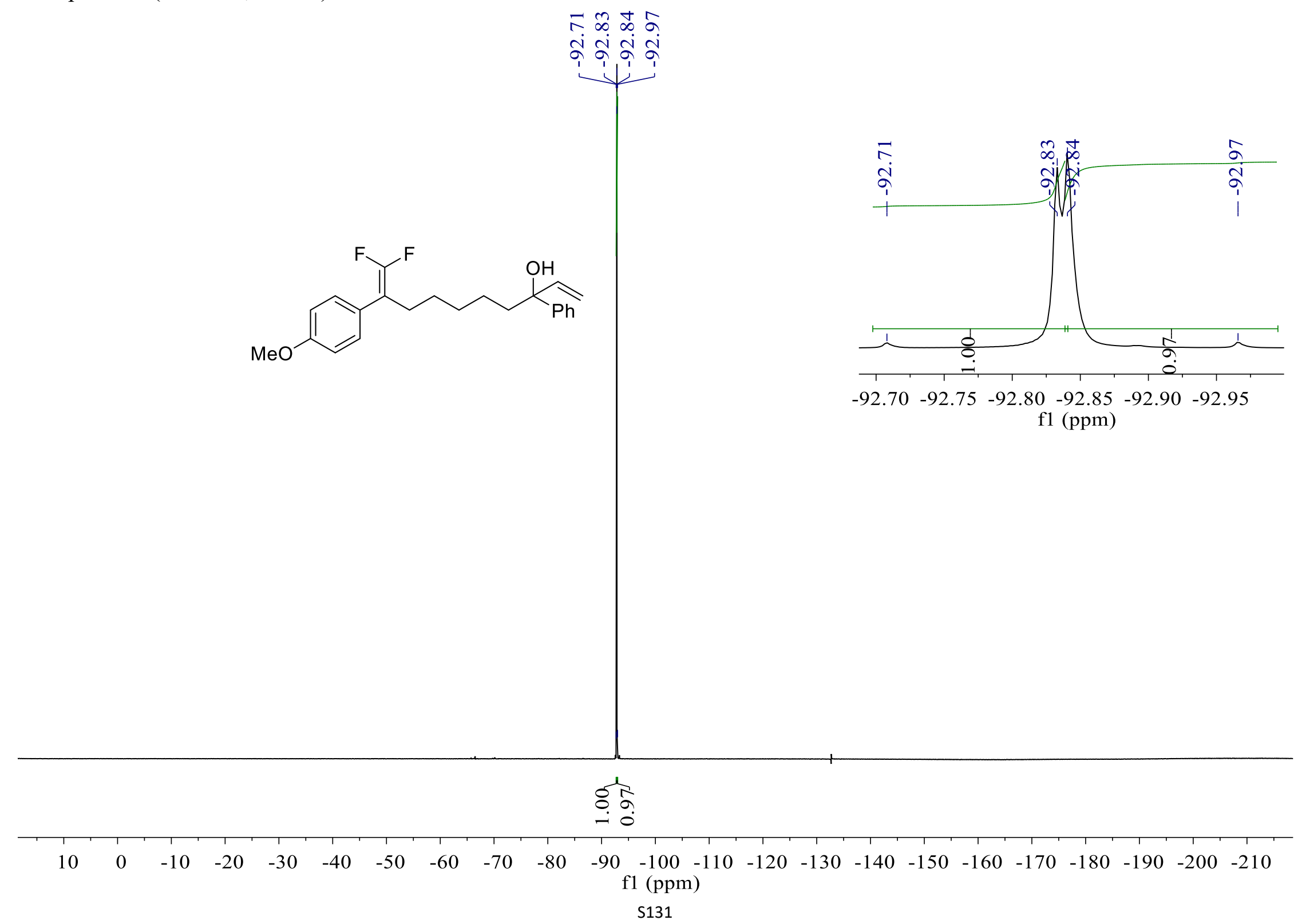




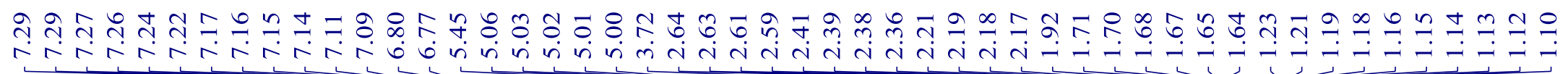

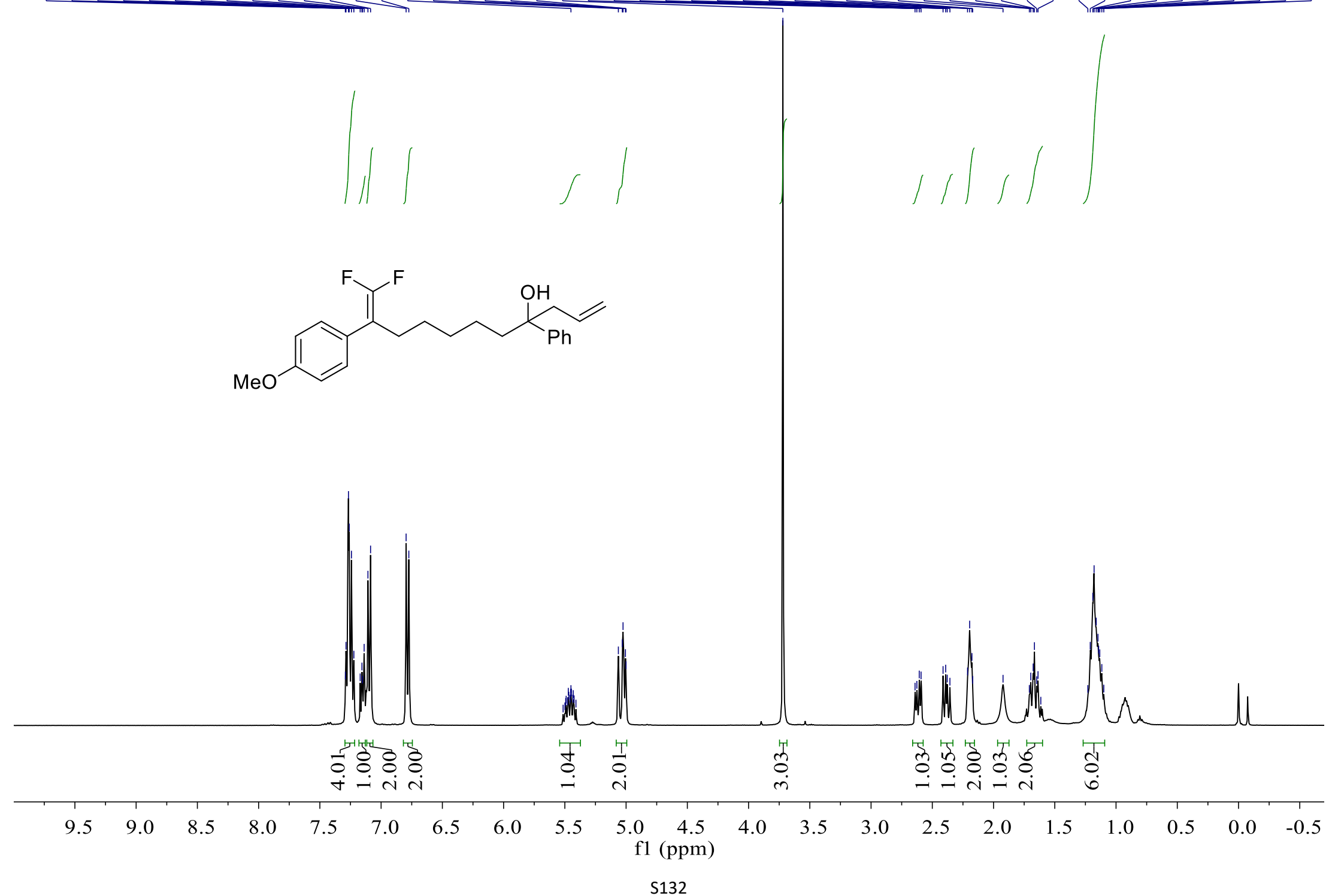


${ }^{13} \mathrm{C}\left\{{ }^{1} \mathrm{H}\right\}$ NMR-spectrum $\left(101 \mathrm{MHz}, \mathrm{CDCl}_{3}\right)$ of $\mathbf{4 c}$

के पे

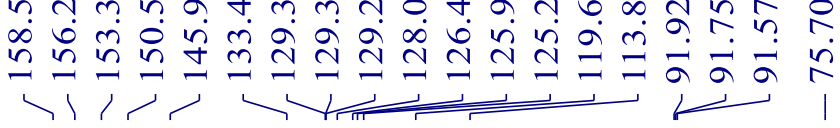

ป⿻ำ

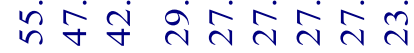

,$<1$ 约

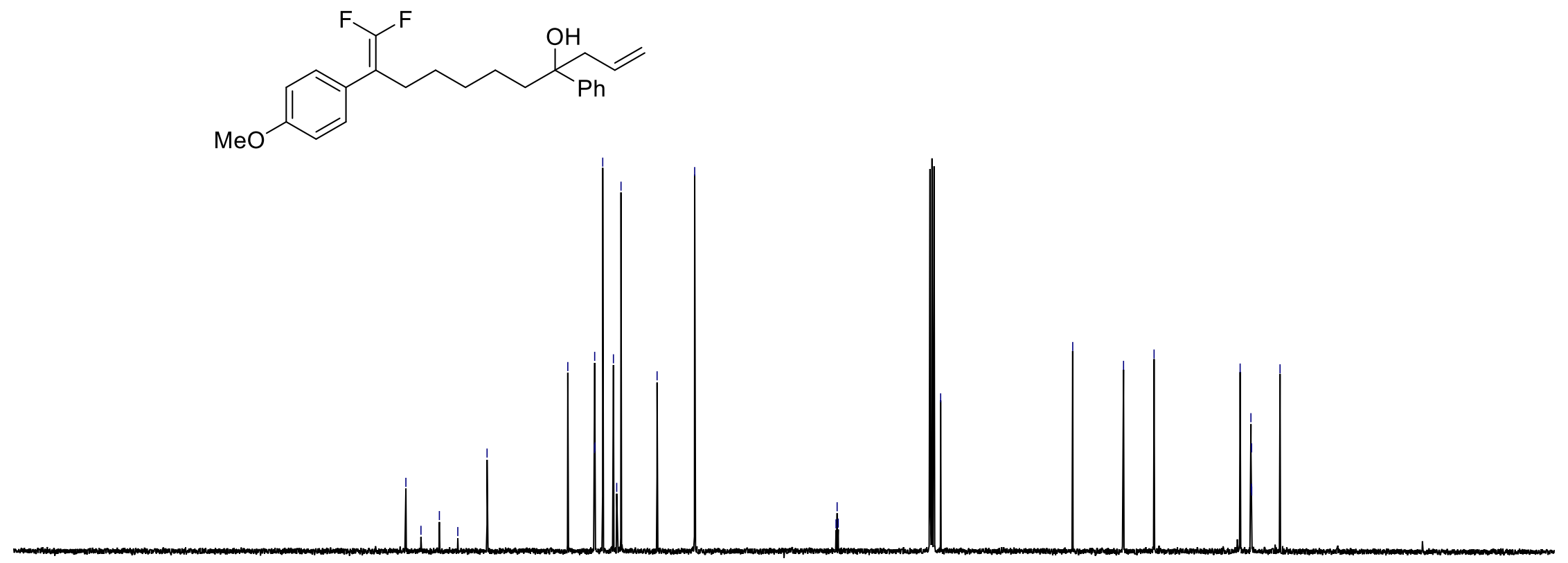

$\begin{array}{cccccccccccccccccccccccc}210 & 200 & 190 & 180 & 170 & 160 & 150 & 140 & 130 & 120 & 110 \begin{array}{c}100 \\ \mathrm{f} 1(\mathrm{ppm})\end{array} & 90 & 80 & 70 & 60 & 50 & 40 & 30 & 20 & 10 & 0 & -10\end{array}$ S133 
${ }^{19} \mathrm{~F}$ NMR-spectrum $\left(376 \mathrm{MHz}, \mathrm{CDCl}_{3}\right)$ of $\mathbf{4 c}$

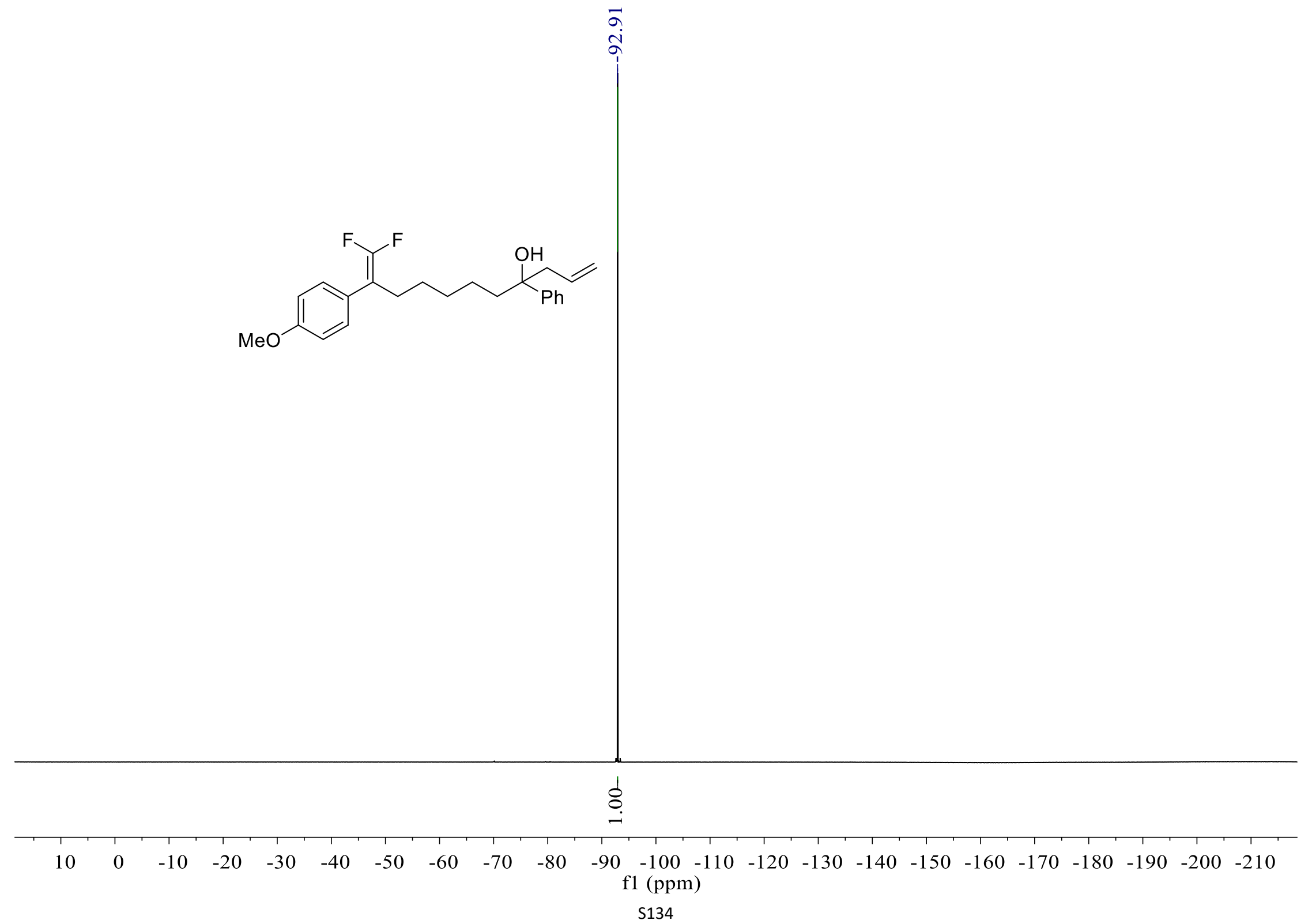




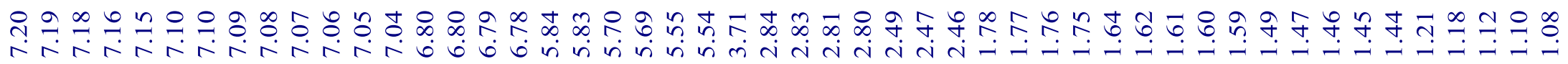

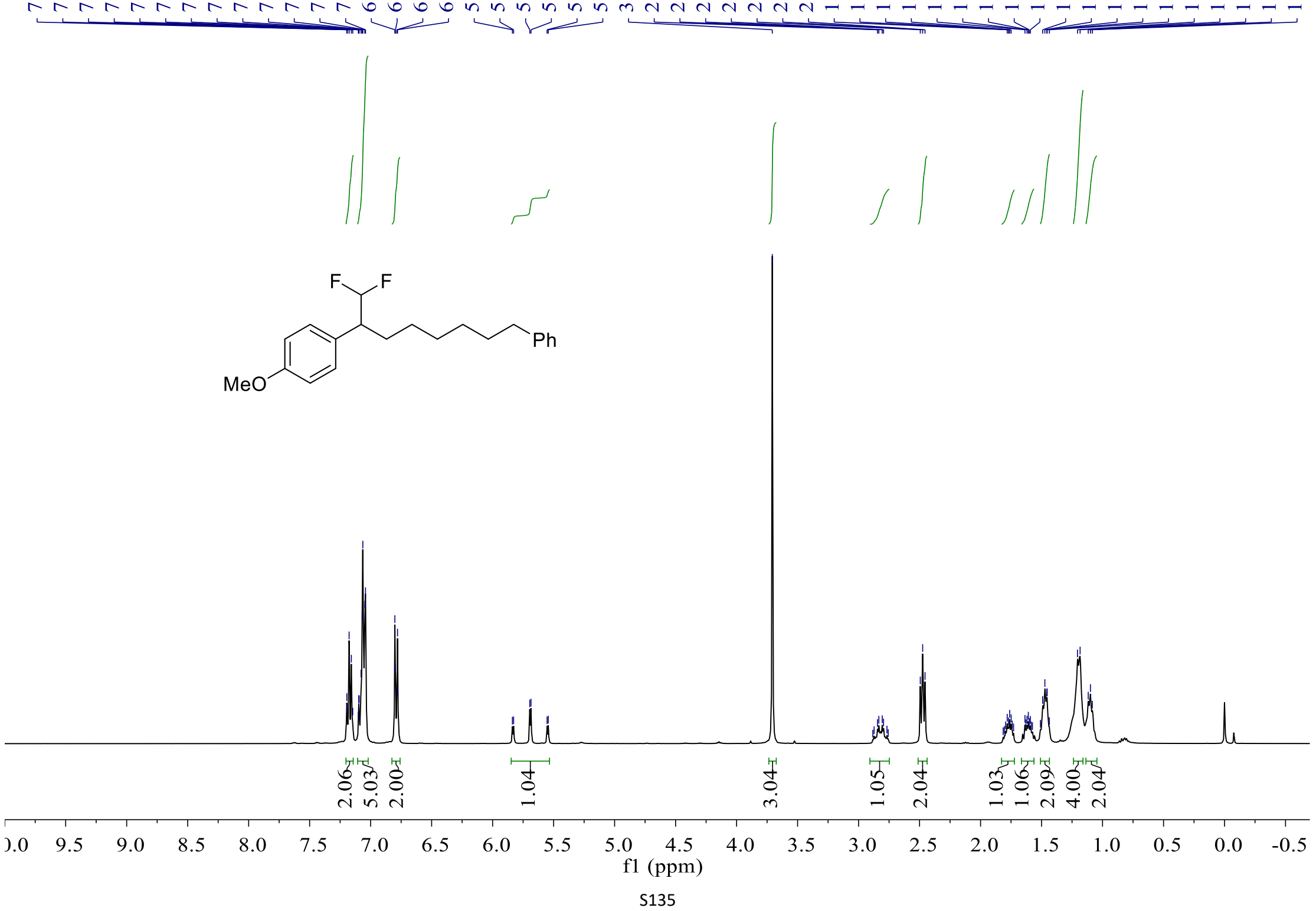


${ }^{13} \mathrm{C}\left\{{ }^{1} \mathrm{H}\right\}$ NMR-spectrum $\left(126 \mathrm{MHz}, \mathrm{CDCl}_{3}\right)$ of $\mathbf{5}$

$$
\begin{aligned}
& \text { ค }
\end{aligned}
$$

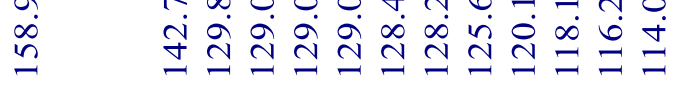

$$
\begin{aligned}
& \text { ปั่ }
\end{aligned}
$$

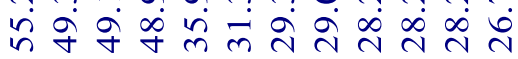
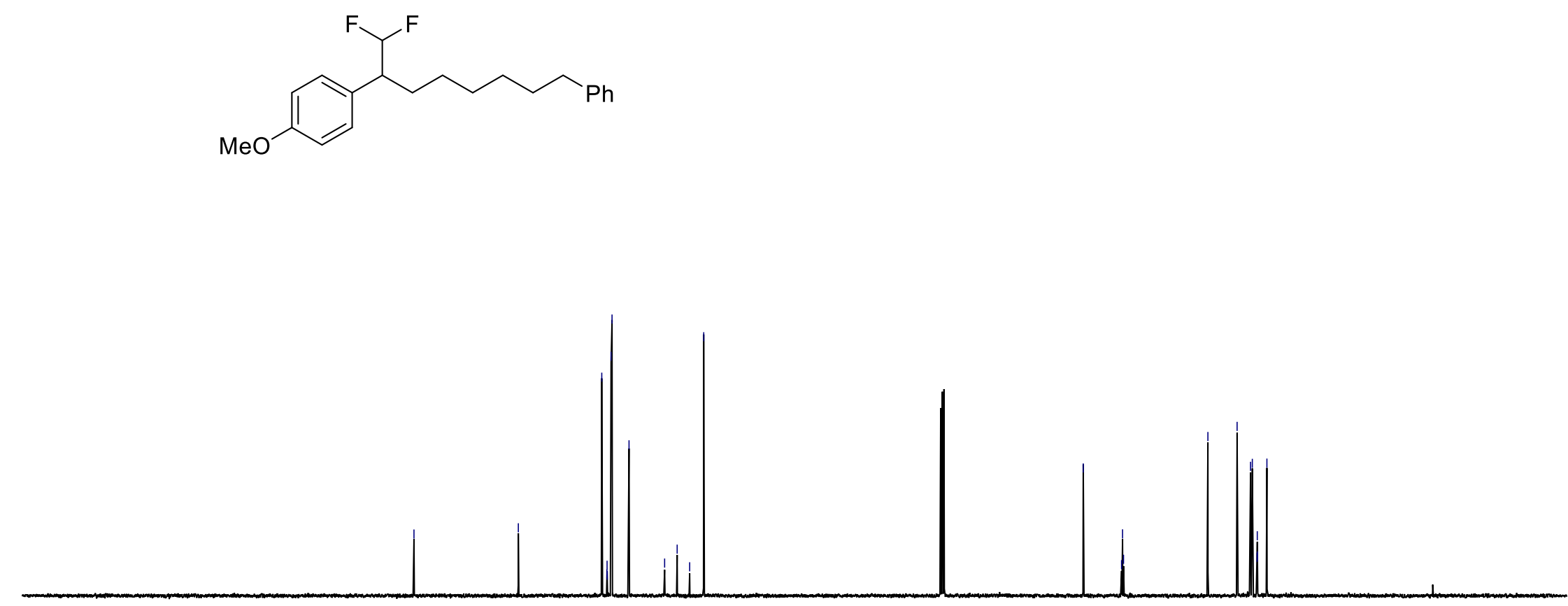

$\begin{array}{rrrrrrrrrrr}210 & 200 & 190 & 180 & 170 & 160 & 150 & 140 & 130 & 120 & 110 \begin{array}{l}100 \\ \text { f1 }(\mathrm{ppm})\end{array}\end{array}$

S136 
${ }^{19} \mathrm{~F}$ NMR-spectrum $\left(376 \mathrm{MHz}, \mathrm{CDCl}_{3}\right)$ of $\mathbf{5}$

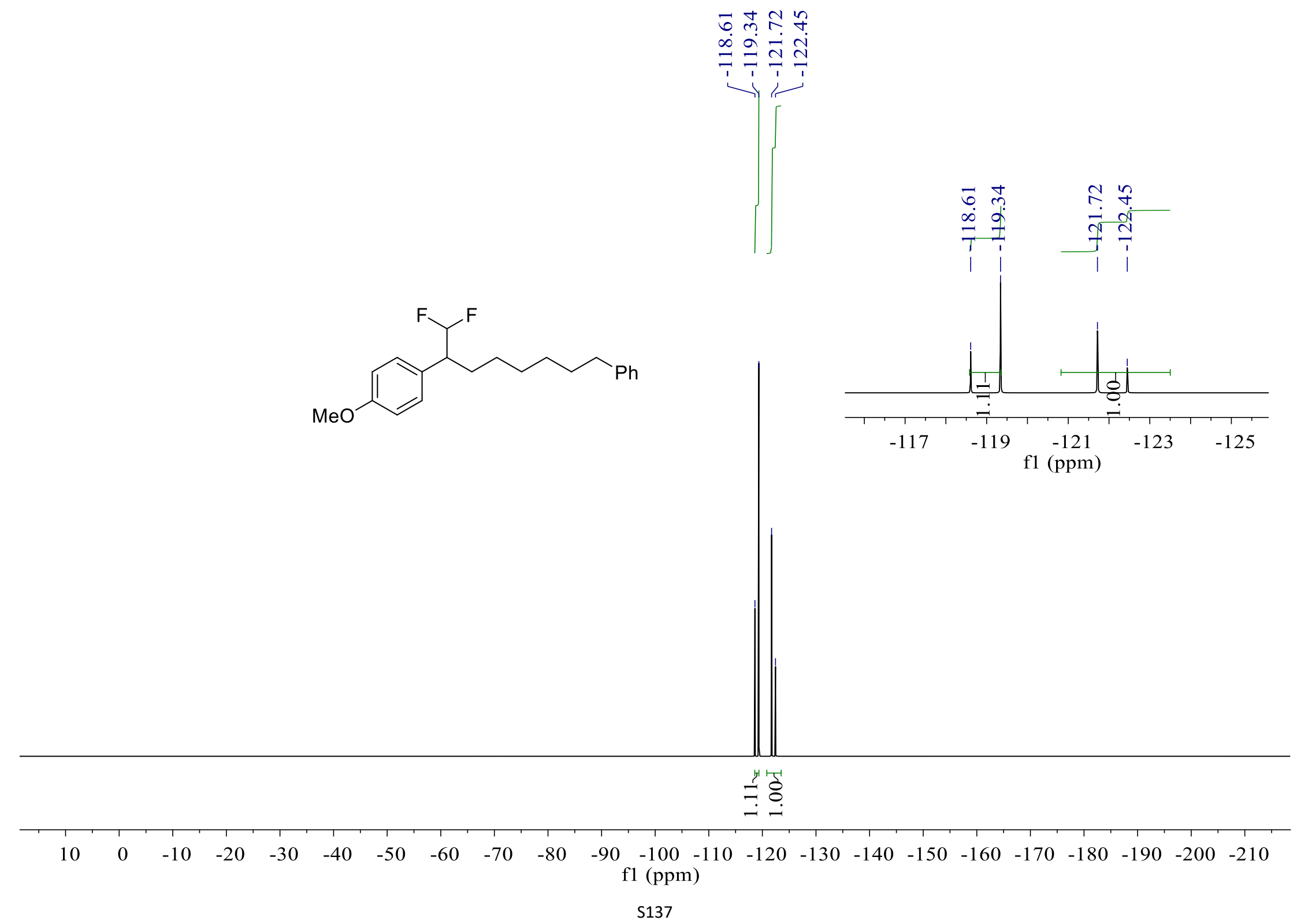




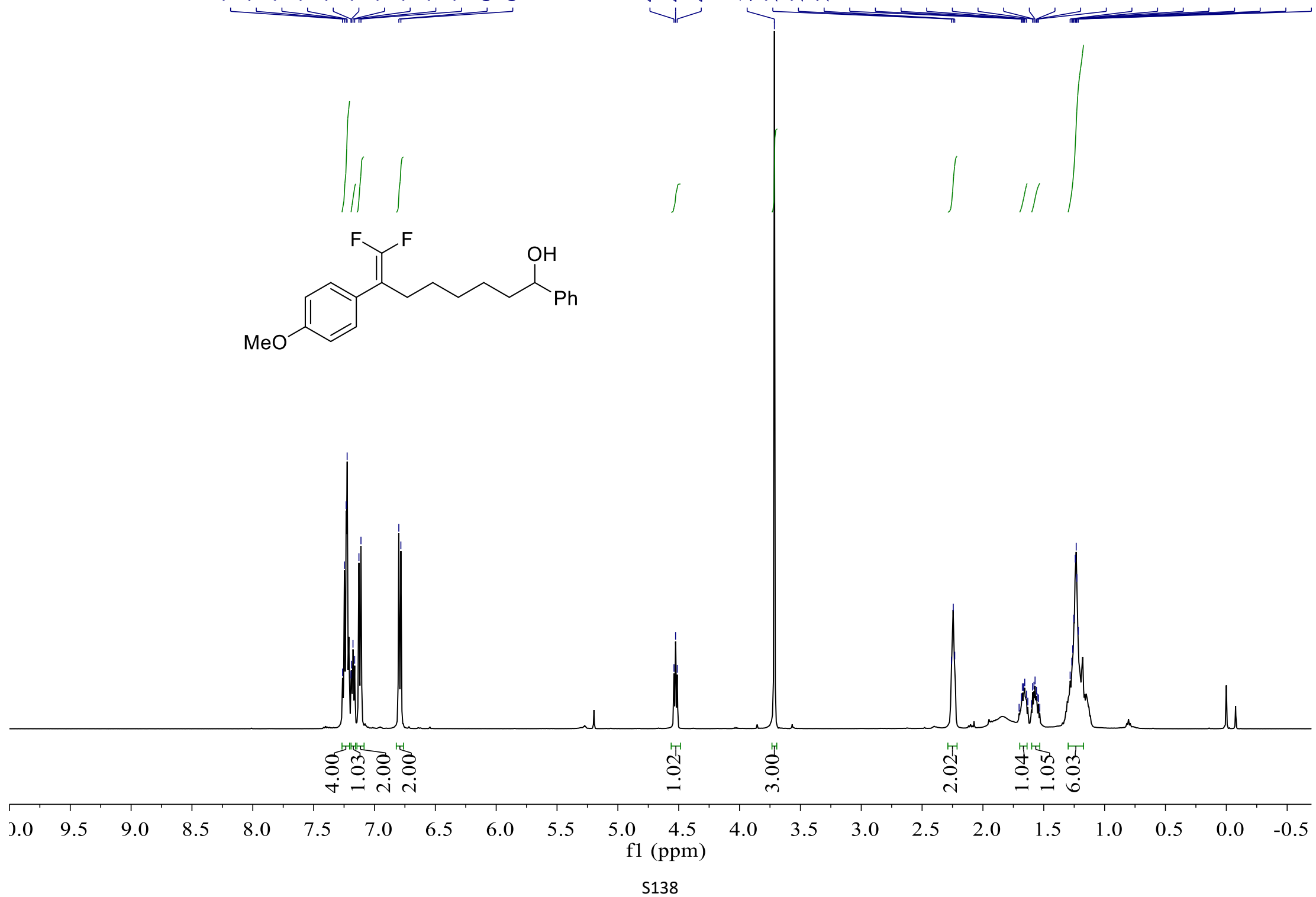

サंர 
${ }^{13} \mathrm{C}\left\{{ }^{1} \mathrm{H}\right\}$ NMR-spectrum $\left(126 \mathrm{MHz}, \mathrm{CDCl}_{3}\right)$ of $\mathbf{6}$

ํํำำ

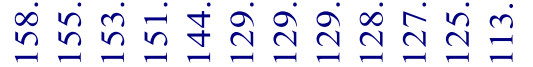

च ।

ลูก बें

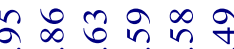

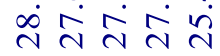

$\sqrt{4 \pi y}$

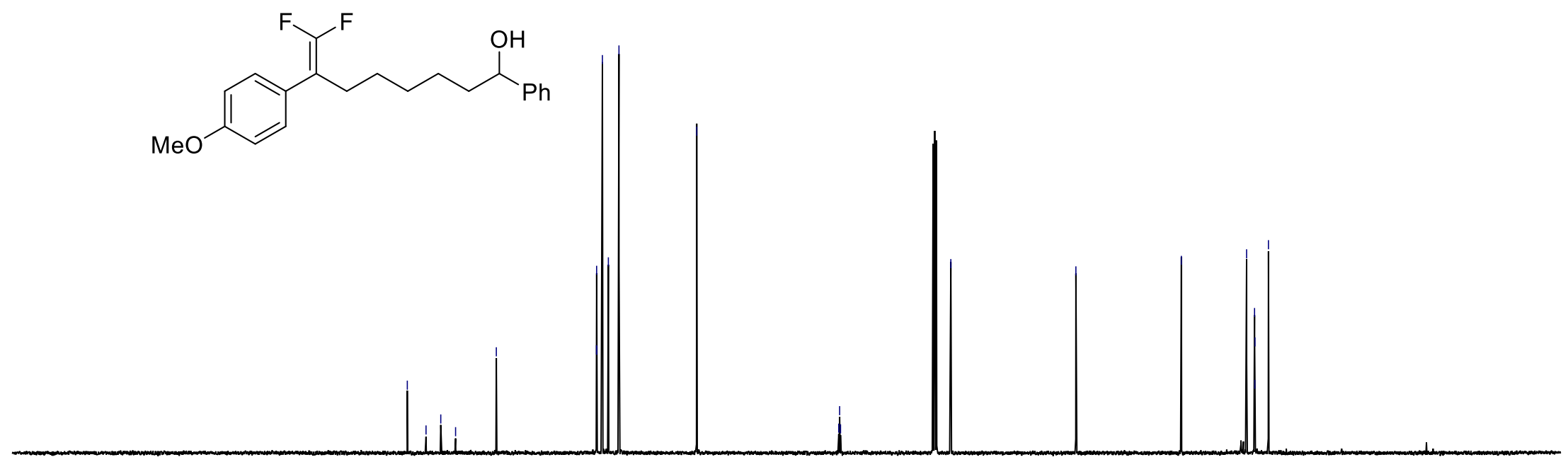

$\begin{array}{lllllllllllll}210 & 200 & 190 & 180 & 170 & 160 & 150 & 140 & 130 & 120 & 110 & 100\end{array}$

f1 (ppm)

S139 
${ }^{19} \mathrm{~F}$ NMR-spectrum $\left(376 \mathrm{MHz}, \mathrm{CDCl}_{3}\right)$ of $\mathbf{6}$

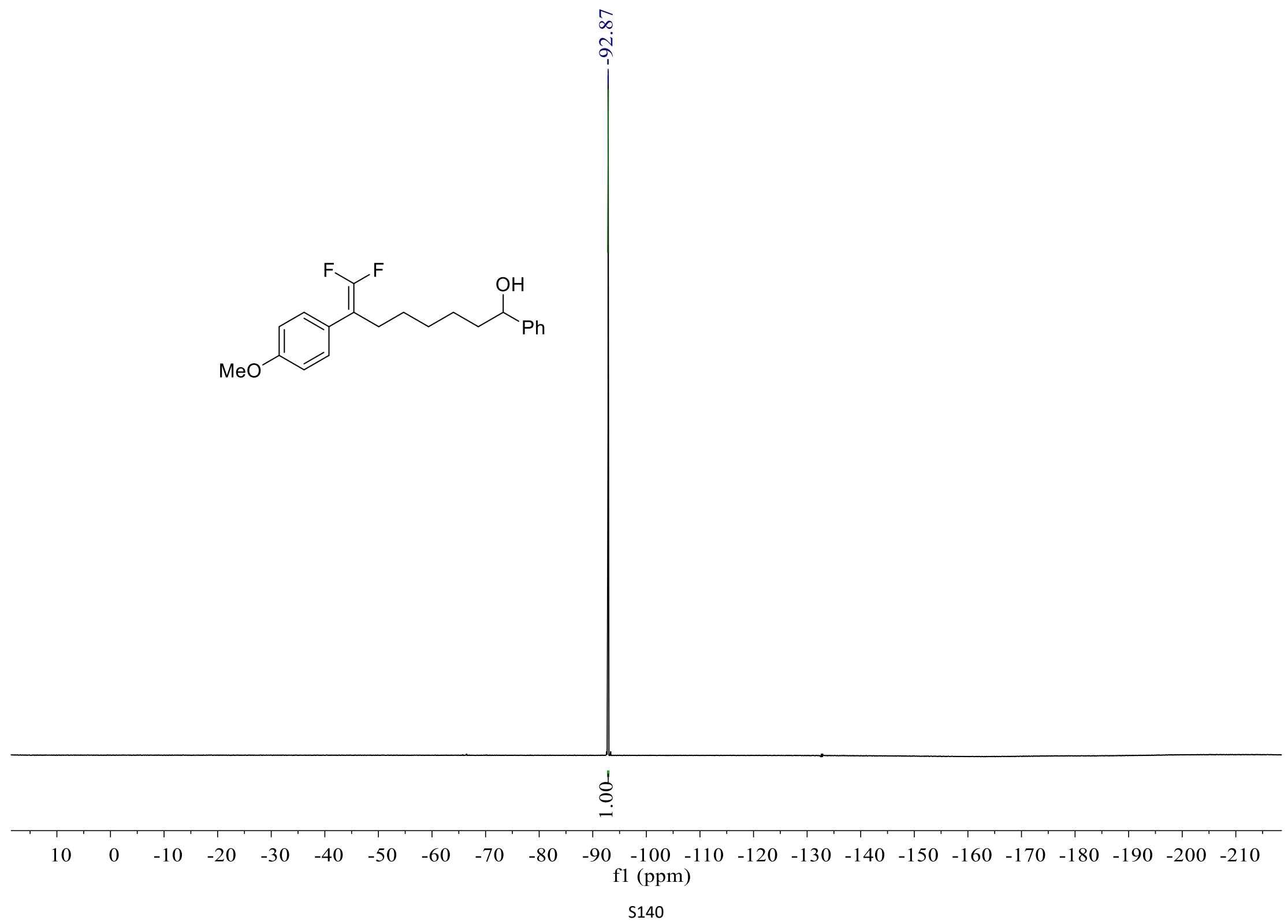




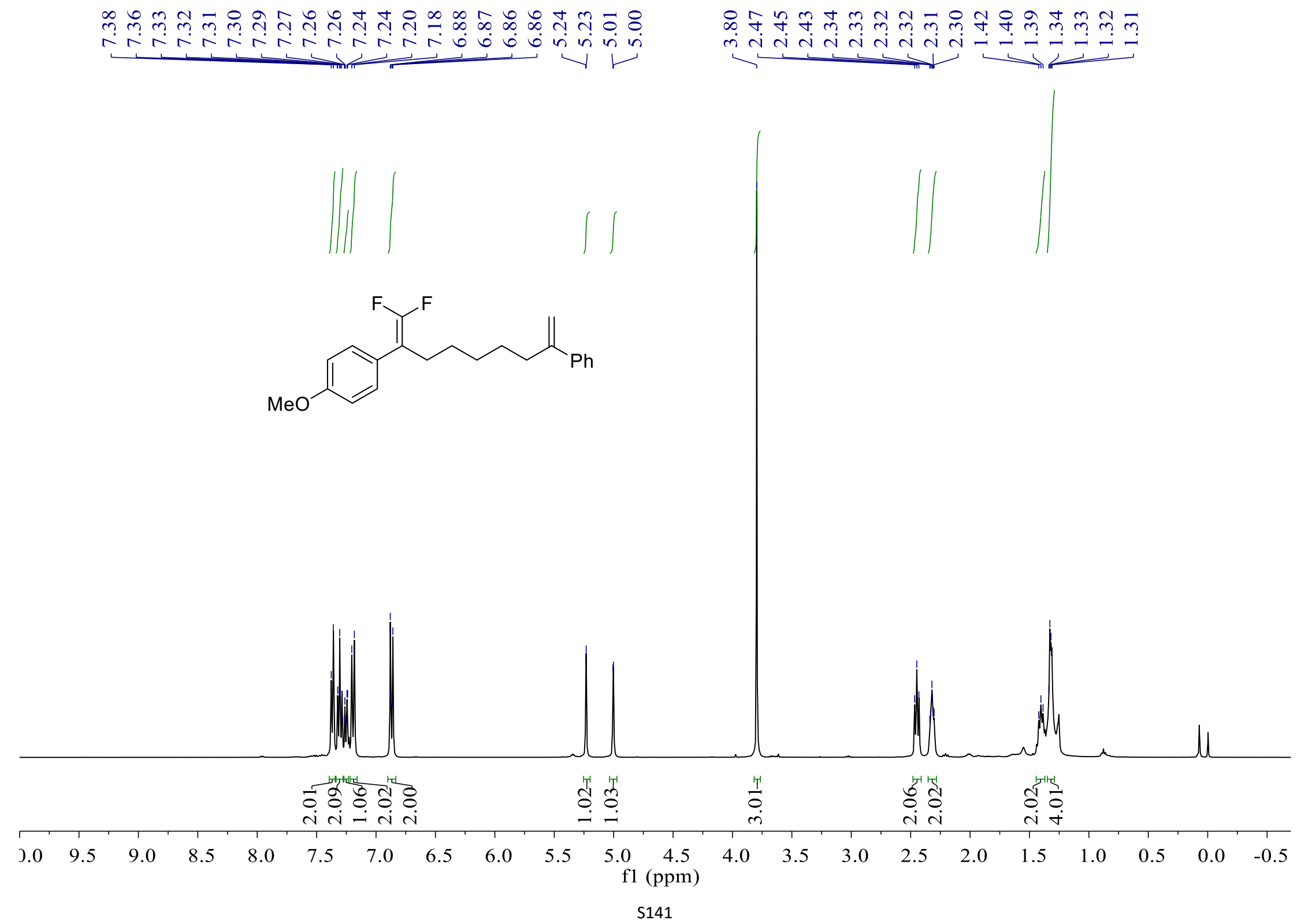


${ }^{13} \mathrm{C}\left\{{ }^{1} \mathrm{H}\right\}$ NMR-spectrum $\left(101 \mathrm{MHz}, \mathrm{CDCl}_{3}\right)$ of 7

\begin{tabular}{|c|c|c|c|}
\hline 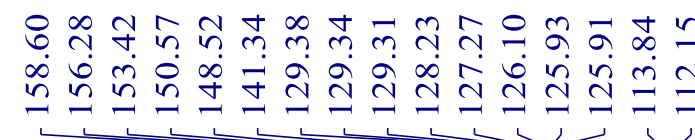 & 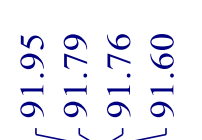 & 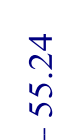 & 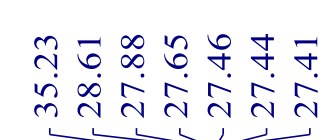 \\
\hline
\end{tabular}

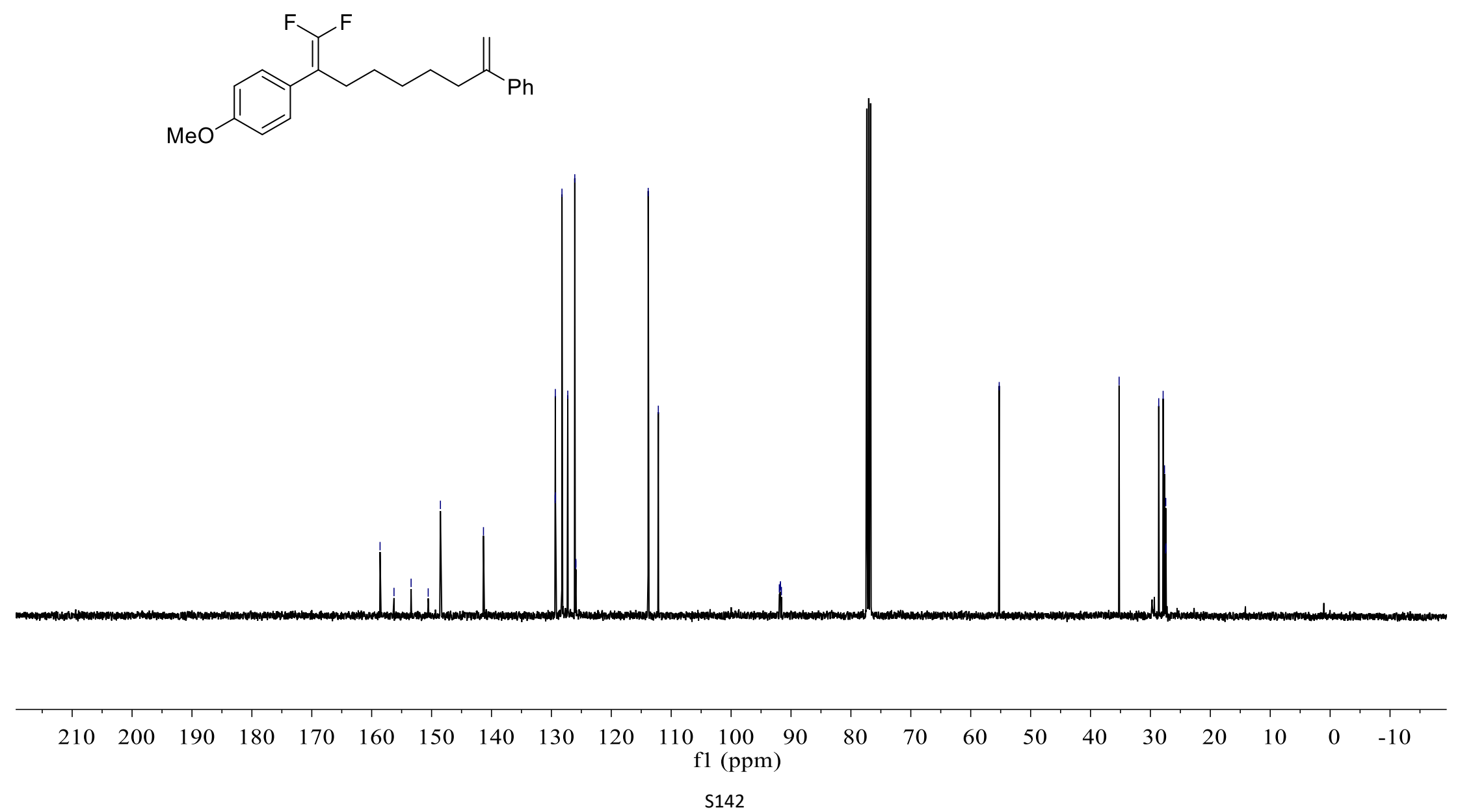


${ }^{19} \mathrm{~F}$ NMR-spectrum $\left(376 \mathrm{MHz}, \mathrm{CDCl}_{3}\right.$ ) of 7

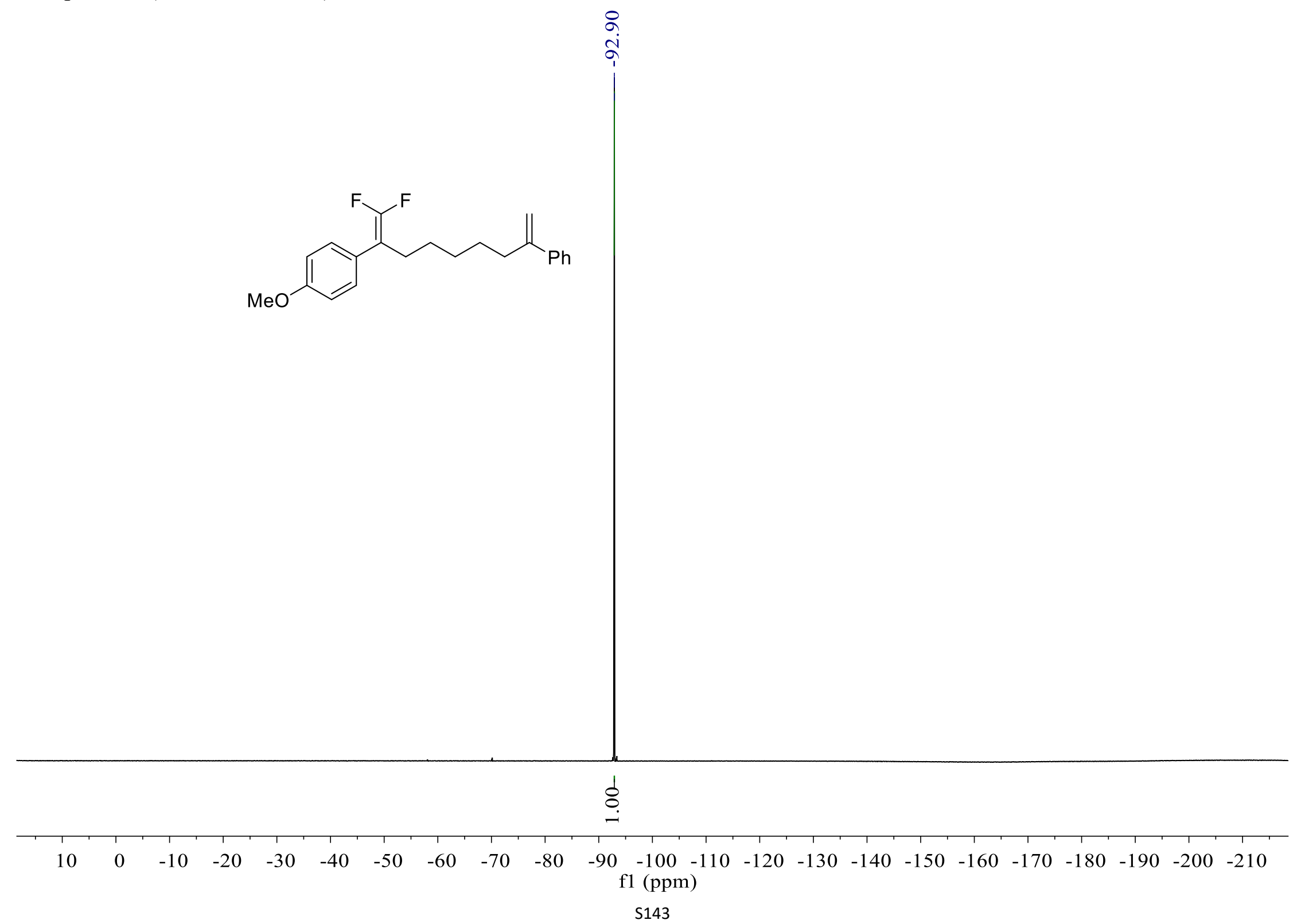




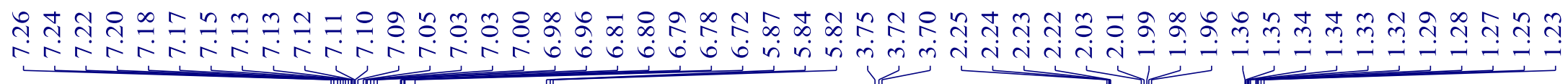
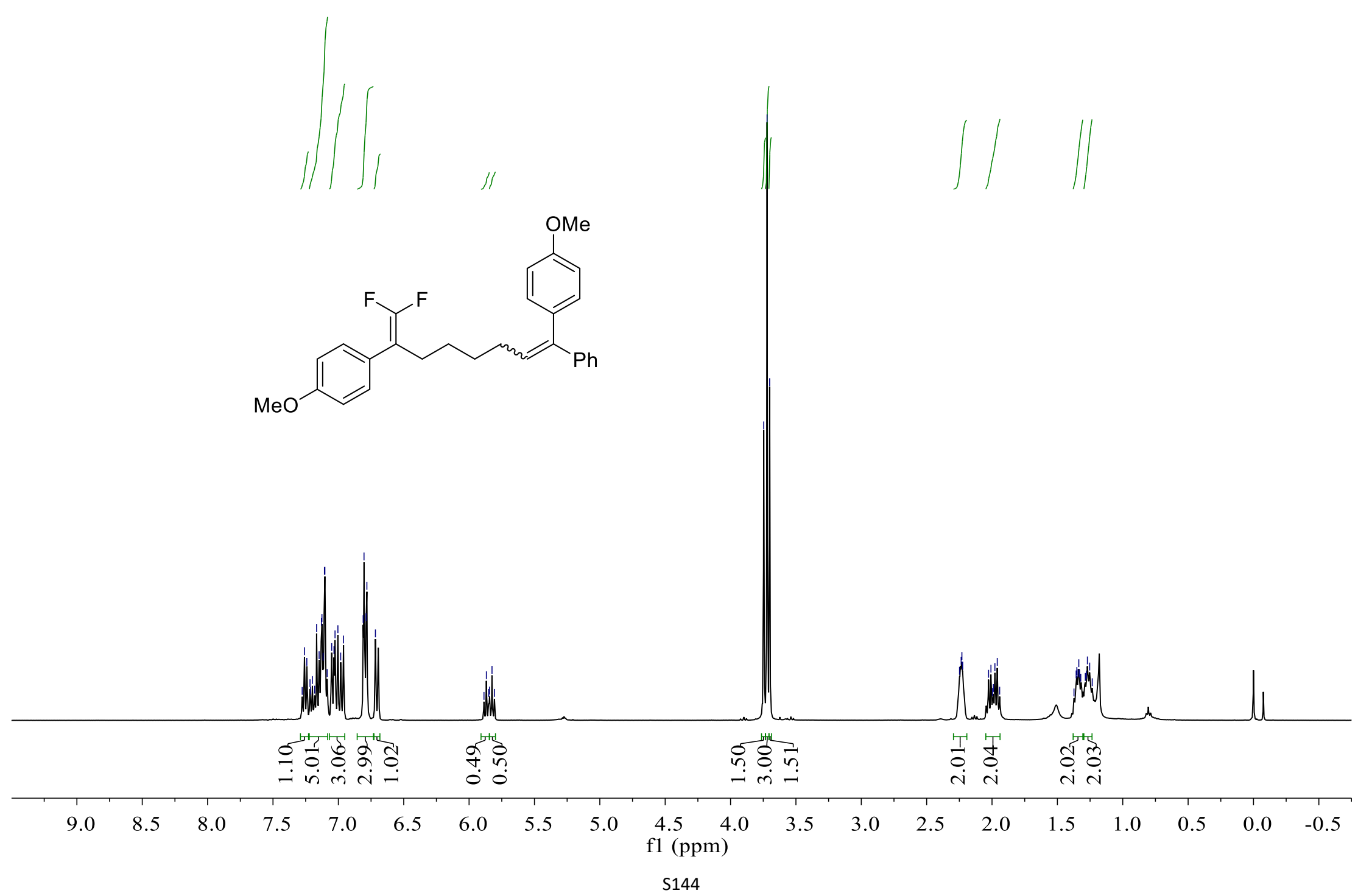
${ }^{13} \mathrm{C}\left\{{ }^{1} \mathrm{H}\right\}$ NMR-spectrum (101 MHz, $\left.\mathrm{CDCl}_{3}\right)$ of 8

ที่

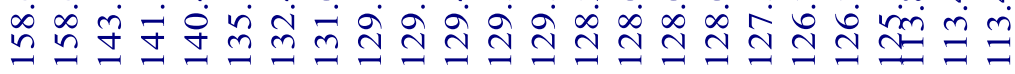

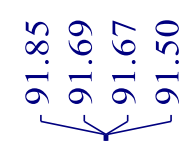

ヘิ ปุ

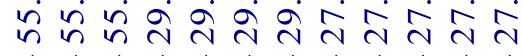

4 W

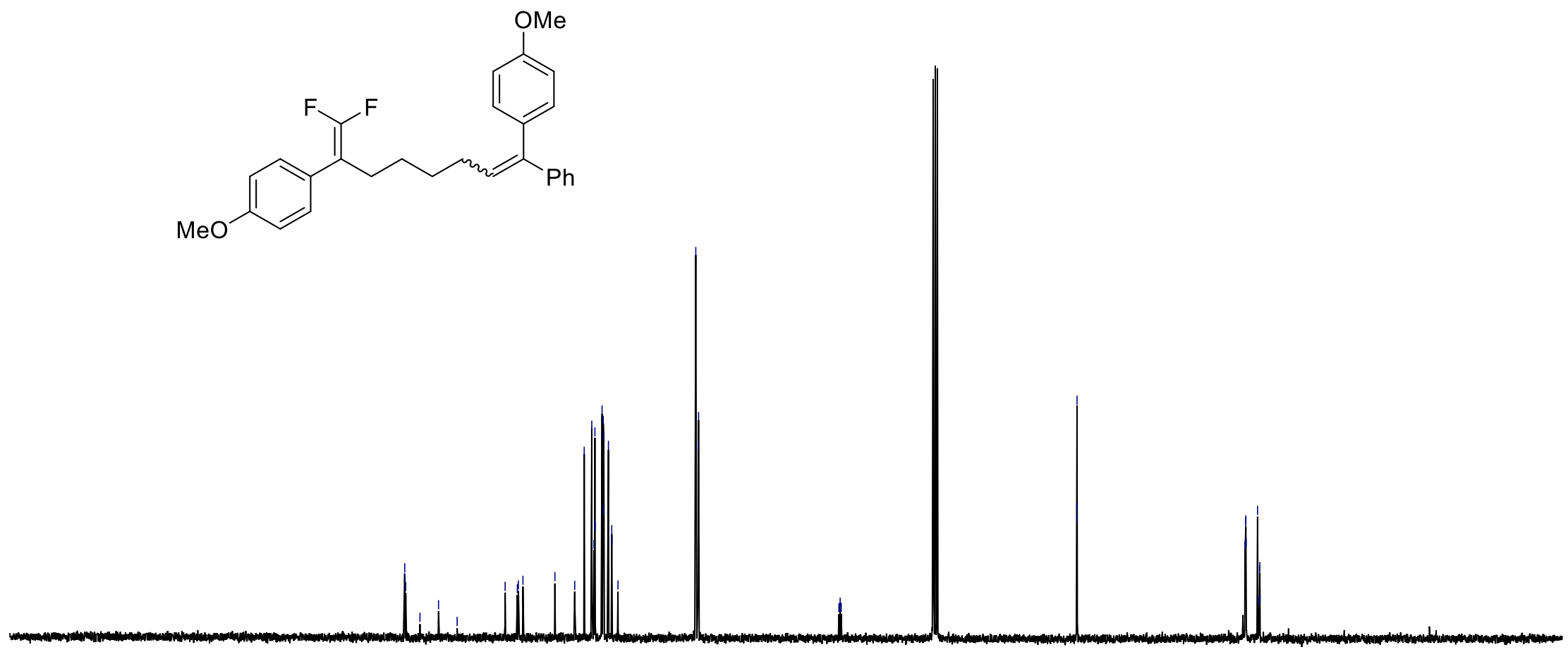

160

$140 \quad 130$

120

110100

f1 (ppm)

60

S145 
${ }^{19} \mathrm{~F}$ NMR-spectrum $\left(376 \mathrm{MHz}, \mathrm{CDCl}_{3}\right.$ ) of 8

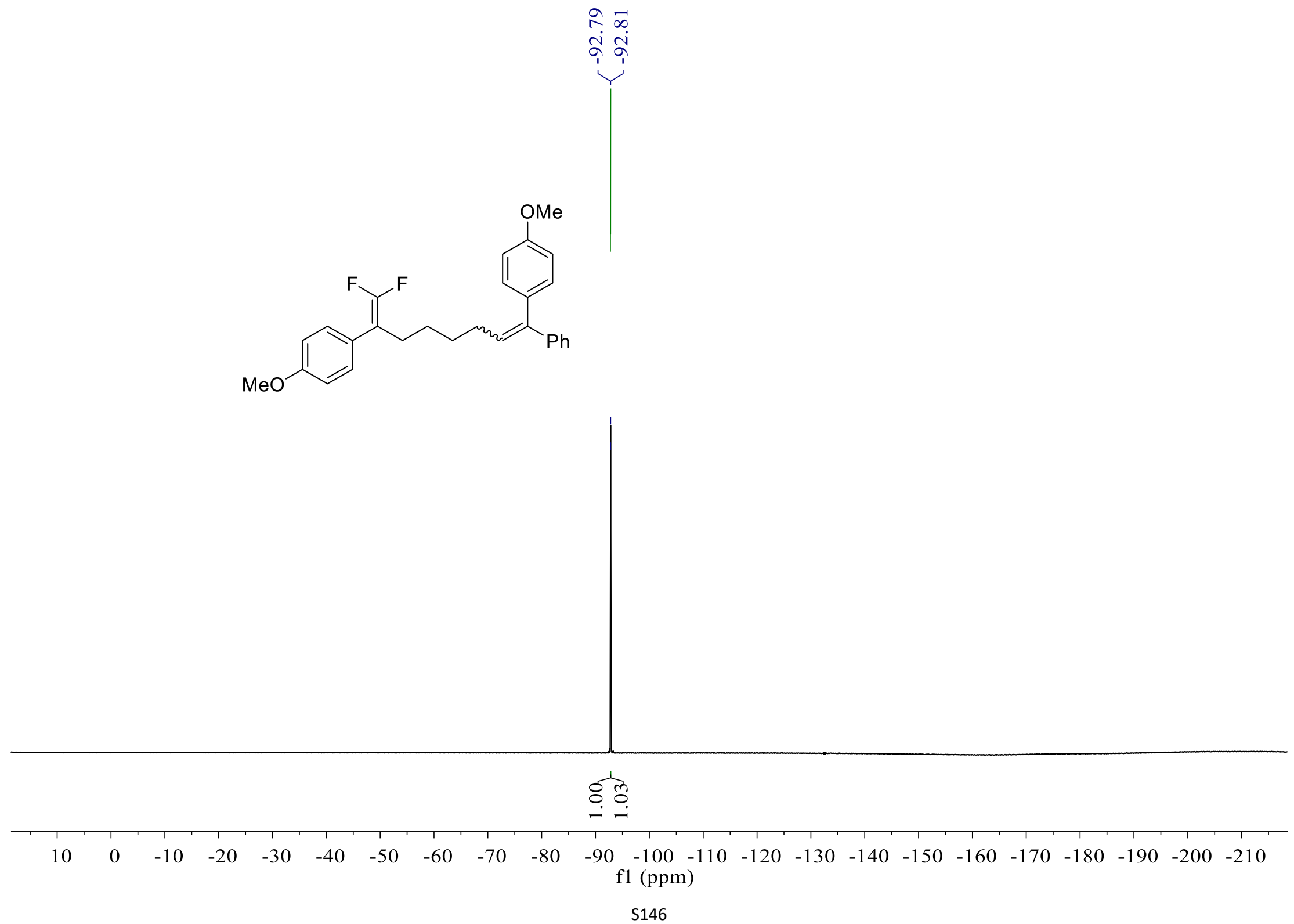

